\title{
Problema da árvore geradora de comunicação ótima: variantes, complexidade e aproximação
}

\author{
Santiago Valdés Ravelo
}

TESE APRESENTADA

$\mathrm{AO}$

Instituto de Matemática e Estatística

DA

Universidade DE SÃo PaUlo

PARA

OBTENÇÃO DO TÍTULO

$\mathrm{DE}$

DOUTOR EM CIÊNCIAS

Programa: Pós-Graduação em Ciência da Computação

Orientador: Prof. Dr. Carlos Eduardo Ferreira

Durante o desenvolvimento deste trabalho o autor recebeu auxílio financeiro da CAPES

São Paulo, março de 2016 


\section{Problema da árvore geradora de comunicação ótima: variantes, complexidade e aproximação}

Esta versão da tese contém as correções e alterações sugeridas pela Comissão Julgadora durante a defesa da versão original do trabalho, realizada em 18/02/2016. Uma cópia da versão original está disponível no Instituto de Matemática e Estatística da Universidade de São Paulo.

Comissão Julgadora:

- Prof. Dr. Carlos Eduardo Ferreira (orientador) - IME-USP

- Pós Doc Aritanan Borges Garcia Gruber - IME-USP

- Prof. Dr. Orlando Lee - UNICAMP

- Prof. Dr. Rafael Crivellari Saliba Schouery - UNICAMP

- Prof. Dr. Luis Augusto Angelotti Meira - UNICAMP 


\section{Agradecimentos}

Primeiro quero agradecer ao meu orientador, o professor Carlinhos, pela ajuda, guia, dicas e observações durante todo processo de doutorado e na obtenção dos resultados que aqui apresento. Também lhe agradeço muito a paciência que teve comigo durante estes cinco anos de estudo.

Sou também grato aos professores que tive no curso, principalmente às professoras Yoshiko e Cristina que além de ótimas aulas fizeram muito boas sugestões na qualificação.

Também sou grato ao IME e à CAPES pelo apoio financeiro nos primeiros anos do doutorado.

Agradeço aos meus amigos, pela ajuda que me deram em diversos momentos, especialmente à Tixa que sempre acreditou em mim, ao Rafael pelas boas ideias que aportou, ao Júnior que sendo de uma área totalmente diferente leu todo o trabalho me ajudando com a escrita.

Sou muito grato ao meu orientador de mestrado, o professor Cláudio, que me introduziu na área de pesquisa de otimização combinatória.

Agradeço também a meus pais que são responsáveis pela minha formação, a meu irmão que me obriga a ser cada dia melhor no plano pessoal e acadêmico, a minha avó que sempre apoiou os meus estudos e a meus tios, tias e primos por estarem sempre presentes e dispostos a ajudar.

Quero agradecer também aos meus colegas de trabalho e amigos na NEXXTO por incentivarem o meu crescimento profissional, técnico e científico.

Finalmente, quero agradecer a todas as pessoas que de uma forma ou outra me ajudaram, apoiaram ou incentivaram durante este período da minha vida.

Muito obrigado a todos vocês! 


\section{Resumo}

RAVELO, S. V. Problema da árvore geradora de comunicação ótima: variantes, complexidade e aproximação. 2016. 120 f. Tese (Doutorado) - Instituto de Matemática e Estatística, Universidade de São Paulo, São Paulo, 2016.

O problema da árvore geradora de comunicação ótima recebe um grafo com comprimentos não negativos nas arestas e um requerimento não negativo entre cada par de vértices; sendo o objetivo encontrar uma árvore geradora do grafo que minimize o custo de comunicação, que é a soma sobre cada par de vértice da distância entre eles na árvore vezes o requerimento entre eles. Este problema é NP-difícil, assim como vários casos particulares dele. Neste trabalho estudamos algumas variantes deste problema, introduzimos novos casos particulares que são também NP-difíceis e propomos esquemas de aproximação polinomial para alguns deles.

Palavras-chave: Árvore Geradora de Comunicação Ótima, Algoritmos de Aproximação, Otimização Combinatória. 


\section{Abstract}

RAVELO, S. V. Optimum communication spanning tree problem: variants, complexity and approximation. 2016. 120 f. Tese (Doutorado) - Instituto de Matemática e Estatística, Universidade de São Paulo, São Paulo, 2016.

The optimum communication spanning tree problem receives a graph with non-negative lengths over the edges and non-negative requirements for each pair of nodes; being the objective to find a spanning tree of the graph that minimizes the communication cost, which is given by the sum, over each pair of nodes, of the distance, in the tree, between the nodes multiplied by the requirement between them. This problem and several of its particular cases are NP-hard. In this work we study some of the variants, also we introduce new NP-hard particular cases of the problem and propose polynomial approximation schemes for some of them.

Keywords: Optimum Communication Spanning Tree, Approximation Algorithms, Combinatorial Optimization. 


\section{Sumário}

$\begin{array}{ll}\text { Lista de Figuras } & \text { ix }\end{array}$

Lista de Tabelas $\quad$ xi

1 Introdução $\quad 1$

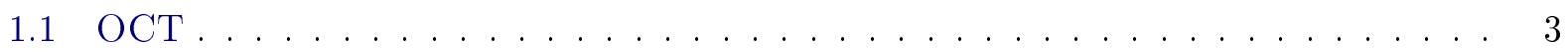

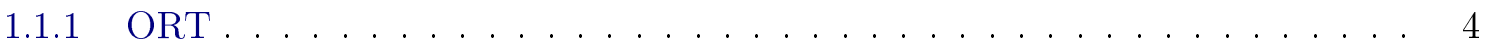

1.1.2 Um caso particular do MRCT métrico . . . . . . . . . . . . . . . 6

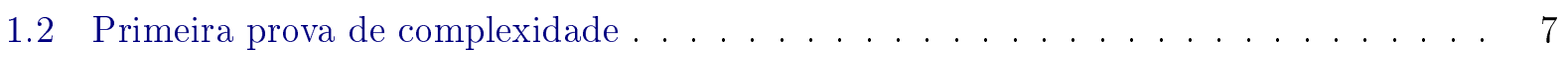

1.3 Redução do MRCT ao caso métrico . . . . . . . . . . . . . . . . . . . . 11

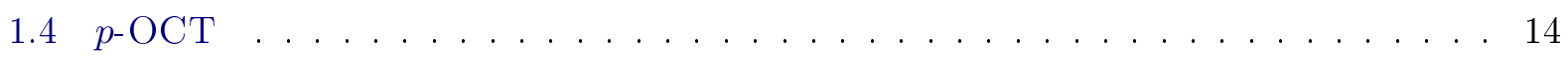

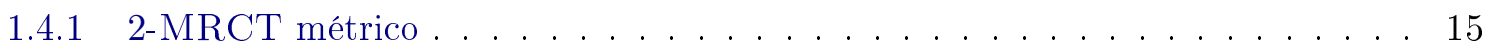

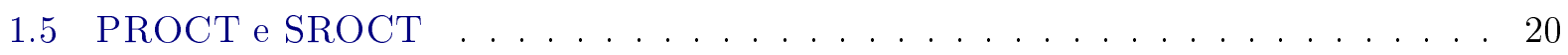

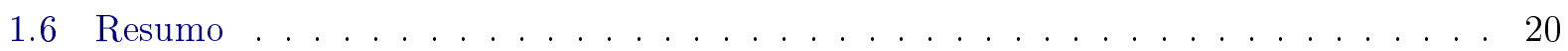

2 Novas variantes do OCT 23

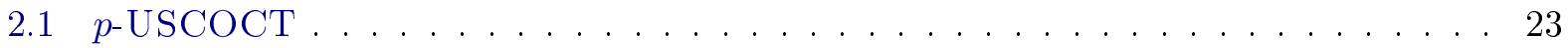

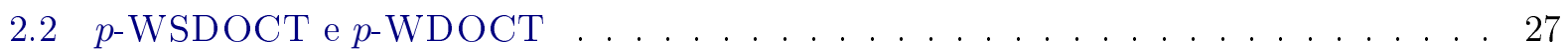

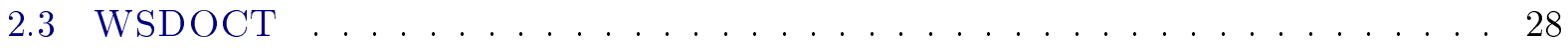

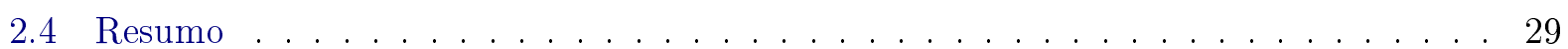

3 Resultados de aproximação na literatura $\quad 31$

3.1 PTAS para o MRCT . . . . . . . . . . . . . . . . . 31

3.2 Aproximação para OCT . . . . . . . . . . . . . . . . . . 31

3.3 Aproximações para o SROCT e o PROCT . . . . . . . . . . . . . 33

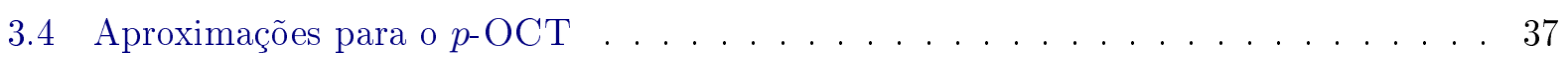

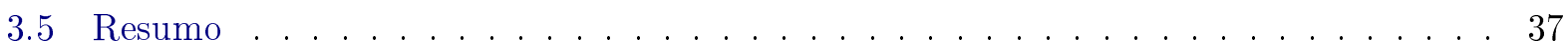

4 PTASs para variantes do $p$-OCT $\quad 39$

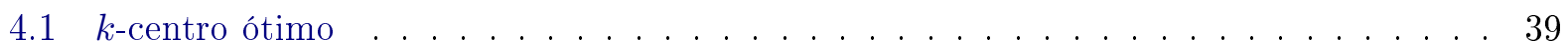

4.2 Lema de aproximação para variantes métricas do $p$-OCT . . . . . . . . . . . . 40

4.3 PTASs para três casos particulares do -OCT métrico . . . . . . . . . . . . . . . . . . . . . . . 45

4.3 .1 PTAS para o $p$-USCOCT métrico . . . . . . . . . . . . 46

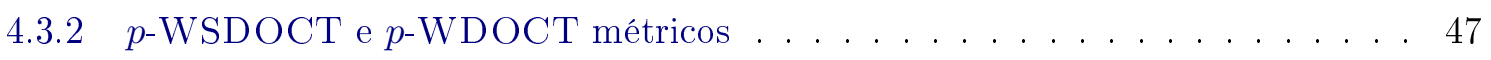

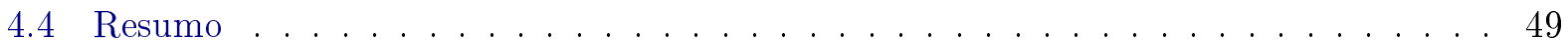


5 Redução do WSDOCT métrico ao problema do $k$-centro ótimo 51

5.1 Definições e notações . . . . . . . . . . . . . . . . . . 51

5.2 Lema de aproximação dado um spine . . . . . . . . . . . . . . . . . 53

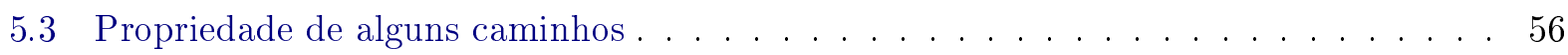

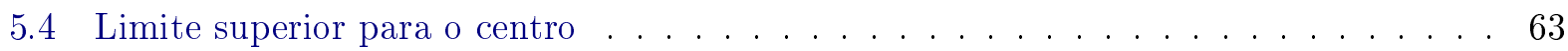

5.5 Limite inferior de uma árvore geradora . . . . . . . . . . . . . . . . 69

5.6 Existência do spine limitado . . . . . . . . . . . . . . . . . . . . 72

5.6.1 Existência de spines de requerimento de envio e de recepção . . . . . . . . . 75

5.7 Teorema de aproximação para WSDOCT métrico $\ldots \ldots \ldots \ldots \ldots$

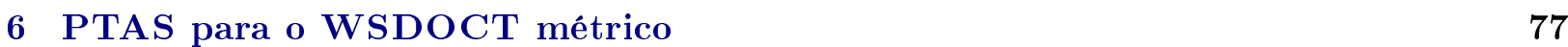

6.1 Razão de aproximação para um $k$-centro ótimo $\ldots \ldots \ldots \ldots$. . . . . . . . 77

$6.2 k$-centro ótimo com requerimentos de recepção inteiros nas folhas . . . . . . . . . . 80

$6.3 k$-centro ótimo com requerimentos de recepção binários nas folhas . . . . . . . . . . . 81

6.4 Resumo . . . . . . . . . . . . . . . . . . . . . . . 84

$\begin{array}{llr}7 & \text { Conclusões } & 87\end{array}$

A Pesquisa a partir de modelos matemáticos $\quad \mathbf{8 9}$

A.1 Modelos matemáticos na literatura . . . . . . . . . . . . . . . . . . 89

A.2 Novos modelos matemáticos . . . . . . . . . . . . . . . . . . . 93

$\begin{array}{ll}\text { Referências Bibliográficas } & 97\end{array}$ 


\section{Lista de Figuras}

1.1 Exemplo de caminho mínimo de mutação entre duas sequências de genomas . . . . . 2

1.2 Exemplo de caminhos mínimos de mutação entre três sequências de genomas . . . . . 2

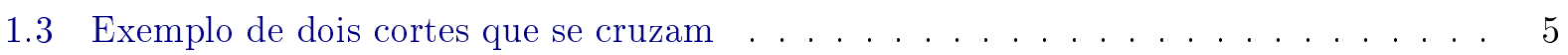

1.4 Exemplo de dois cortes definidos a partir da remoção de arestas de uma árvore . . . 6

1.5 Grafo para MRCT construído a partir de uma instância do 3-EC . . . . . . . . . . 8

1.6 Possíveis casos de eliminar uma aresta ruim de uma árvore enraizada . . . . . . . . . 12

1.7 Grafo para 2-MRCT construído a partir de uma instância do 3-EC . . . . . . . . . 16

1.8 Relação entre o OCT e suas variantes NP-difíceis . . . . . . . . . . . . . . . . . 21

2.1 Grafo para o USCOCT construído a partir de uma instância do SAT . . . . . . . 25

2.2 Relação entre o OCT e suas variantes NP-difíceis . . . . . . . . . . . . . . . . . 29

3.1 Relação, que inclui aproximação, entre o OCT e suas variantes NP-difíceis . . . . . . 38

4.1 Exemplo de notação para $p$-OCT de caminhos em uma árvore geradora . . . . . . . 41

4.2 Relação entre o OCT e suas variantes NP-difíceis . . . . . . . . . . . . . . . . . . 49

5.1 Exemplo de notação para WSDOCT de caminhos em uma árvore geradora $\ldots . . .53$

5.2 Exemplo de um spine de uma árvore geradora de $G \ldots \ldots$. . . . . . . . . . 65

5.3 Exemplo de centro obtido a partir de spine de uma árvore geradora . . . . . . . . 66

5.4 Exemplo de criação de um spine a partir de dois conjuntos de caminhos em que exatamente um par de caminhos têm vértices internos em comum e não têm extremos em comum . . . . . . . . . . . . . . . . . . . . 73

5.5 Exemplo de criação de um spine a partir de dois conjuntos de caminhos em que os caminhos têm interseção que inclui os extremos . . . . . . . . . . . . . . . . . . 74

6.1 Relação entre o OCT e suas variantes NP-difíceis . . . . . . . . . . . . . . . . . 85 


\section{Lista de Tabelas}

3.1 Resumo dos resultados de aproximação na literatura . . . . . . . . . . . . . . 37

4.1 Comparação dos resultados para $p$-OCT com os da literatura . . . . . . . . . . . 50 


\section{Capítulo 1}

\section{Introdução}

Grafos são uma fonte inesgotável de problemas com enunciados simples mas que escondem, em algumas ocasiões, uma sofisticada estrutura matemática. Muitas vezes esses problemas são de grande interesse teórico além de estarem presentes em diversas aplicações do mundo real. Considere, por exemplo, uma companhia de telefonia que deseja construir cabos de transmissão ligando cidades. Se conhecemos os custos de construir as linhas de transmissão entre elas e o objetivo da companhia é encontrar a rede que conecte as cidades de forma que o custo de construção seja mínimo, então o problema é o de encontrar a árvore geradora de custo mínimo para o qual conhecemos algoritmos eficientes que o resolvem de forma exata. Já se mudarmos um pouco a aplicação o problema pode ficar muito difícil. Por exemplo, se soubéssemos para cada par de cidades a comunicação esperada entre elas, e desejássemos encontrar um conjunto de ligações que minimizem o custo de comunicação que é dado pela soma, para cada par de cidades, do produto da comunicação esperada entre elas pela distância que as separa, então este problema é NP-difícil ([JLRK78]). Este é o chamado problema da árvore geradora de comunicação ótima (OCT, optimum comunication spanning tree problem) introduzido originalmente por $\mathrm{Hu}$ em 1974 ([Hu74]) que é o problema central deste trabalho.

De forma mais precisa, no problema da árvore geradora de comunicação ótima, é dado um grafo com comprimentos não negativos nas arestas e requerimentos não negativos entre cada par de vértices. O objetivo é encontrar uma árvore geradora do grafo que minimize o custo de comunicação, que é a soma sobre cada par de vértices da distância entre eles na árvore multiplicado pelo requerimento de comunicação entre eles.

Este problema, assim como algumas das suas variantes, encontram diferentes aplicações práticas. Uma destas aplicações aparece em biologia computacional para ajudar na solução de problemas de

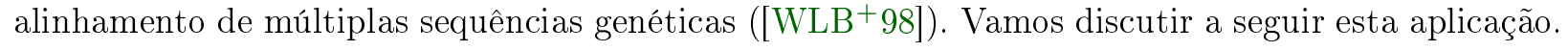

O objetivo desse problema é encontrar padrões comuns em um conjunto de sequências de genomas. Para isto, é usualmente usado o conceito de caminho mínimo de mutação entre as sequências, que é o menor número de caracteres a serem inseridos nelas até que ambas fiquem iguais. A Figura 1.1 dá um exemplo deste caminho entre duas sequências.

No caso de duas sequências, o problema pode ser resolvido eficientemente, pois existem algoritmos de programação dinâmica para solucioná-lo ([Wat95]). Porém, podemos generalizar o problema para permitir um número qualquer de sequências, onde dadas $k$ sequências genéticas se deseja inserir caracteres nelas de forma tal que todas fiquem iguais. O objetivo é minimizar a soma das distâncias de cada par de sequências, que pode ser com pesos quando se deseja que certos pares estejam mais próximos. Considere o exemplo dado pela Figura 1.2 mostra com $k=3$. 


$\begin{array}{ccccccccc}s_{1}: & A & T & T & C & G & A & & A \\ & A & T & T & C & G & A & T & A \\ & A & T & T & C & G & A & T & A \\ & A & & T & C & G & A & T & A \\ s_{2}: & A & & T & C & G & & T & A\end{array}$

Figura 1.1: Considere as sequências de genoma $s_{1}=$ ATTCGAA e $s_{2}=$ ATCGTA. Observe que o comprimento de um caminho mínimo de mutação entre elas é igual a 3, isto se pode obter inserindo o caractere $T$ na penúltima posição de $s_{1}$ e inserindo em $s_{2}$ os caracteres $T$ na segunda posição e A na antepenúltima.

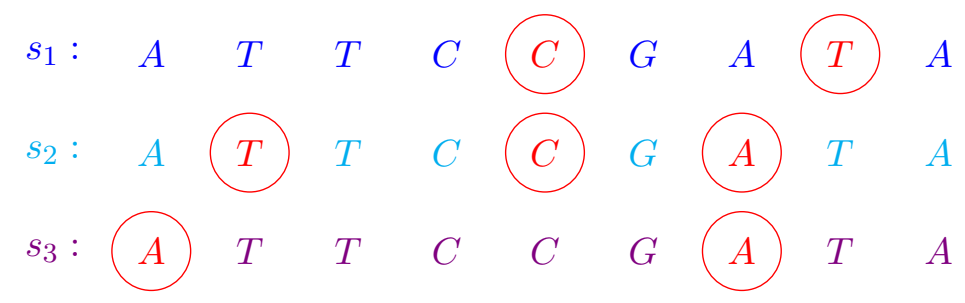

Figura 1.2: Neste exemplo são dadas as sequências de genoma: $s_{1}=$ ATTCGAA, $s_{2}=$ ATCGTA $e$ $s_{3}=$ TTCCGTA. Observe que o comprimento do caminho mínimo de mutação entre $s_{1}$ e $s_{2}$ é igual a 3 , entre $s_{1}$ e $s_{3}$ é igual a 4 e entre $s_{2}$ e $s_{3}$ é igual a 3.

Observe que este problema pode ser formulado como um problema de árvore geradora de comunicação ótima. Para isto, podemos considerar que os vértices são os genomas e o custo da aresta que liga dois genomas é dado pelo número mínimo de caracteres introduzidos que transformam as sequências dos genomas até que fiquem iguais. Já o requerimento de comunicação entre os genomas pode modelar a prioridade de aproximar cada par e caso esta não exista ele é o mesmo para todos os pares.

Uma outra aplicação prática, mencionada anteriormente, encontra-se no planejamento de redes de telecomunicações ou transporte ([Con09, CFM10]). Por exemplo, para garantir uma conexão com maior velocidade e mais resiliente seria interessante implantar um sistema de redes de fibra ótica, porém estas costumam ser mais custosas e em geral é suficiente que exista uma conexão entre dois pontos da rede. Desta forma uma solução teria forma de árvore. Neste tipo de problema pode-se conhecer uma estimativa da quantidade de dados que serão enviados entre cada par de pontos da rede, logo o objetivo seria implantar uma rede que minimize o tráfego de dados nela, isto é, a soma das distâncias entre pontos vezes a quantidade de dados que eles trocam. Não é difícil ver que este tipo de problema pode ser modelado como uma instância do OCT, em que os pontos de rede são vértices, os comprimentos das arestas entre os vértices são dadas pela quantidade de material necessário para conectar dois pontos e o requerimento pela quantidade de informação que eles trocam. Outras aplicações ocorrem em problemas de distribuição de recursos em geral ([AM87b, AM87a]).

Como mencionado anteriormente, o interesse nestes problemas não é somente prático, senão também teórico pois uma grande variedade deles são NP-difíceis, inclusive quando consideramos unicamente grafos métricos ([JLRK78, WLB ${ }^{+} 98, \mathrm{FFK}^{+} 00$, Wu02]). Isto implica que não são conhe- 
cidos algoritmos eficientes para solucionar os mesmos e, a menos que $\mathrm{P}=\mathrm{NP}$, tais algoritmos não existem. Por esse motivo vários trabalhos se dedicaram ao estudo de aproximações para estes problemas ([WLB ${ }^{+} 98$, WCT00a, WCT00b, Wu02, Wu04]), outros tentaram modelagens matemáticas para estudar diferentes características destes problemas ([Rot07, Con09, CFM10]), e claro, também houve vários algoritmos heurísticos e meta-heurísticos propostos ([AM87b, AM87a, Sha06, Rot07, Rot09]).

Portanto, nosso objetivo neste trabalho é estudar estes problemas, principalmente suas versões métricas, e propor algoritmos de aproximação para alguns deles melhorando vários dos resultados existentes na literatura.

A tese é organizada como segue: nas seguintes seções deste capítulo apresentamos formalmente o OCT e algumas de suas variantes tratadas na literatura, fazendo uma revisão bibliográfica dos principais resultados sobre a complexidade deles. Específicamene, na Seção 1.1 apresentamos a definição formal do OCT e discutimos sobre os resultados apresentados por Hu em 1974 ([Hu74]) para dois casos particulares dele. Na Seção 1.2 mostramos o primeiro resultado que aparece na literatura sobre a complexidade do OCT, dado por Johnson et al. em 1978 ([JLRK78]) para um caso particular dele chamado MRCT. Na Seção 1.3 analisamos parte dos resultados apresentados por Wu et al. em 1998 ([WLB $\left.{ }^{+} 98\right]$ ), em que se prova que estes problemas são difíceis inclusive quando somente consideramos grafos métricos. Na Seção 1.4 apresentamos um outro caso particular do OCT, o $p$-OCT e ilustramos uma das diferentes provas sobre a complexidade deste que aparecem na literatura ([FFK $\left.\left.{ }^{+} 00, \mathrm{Wu} 02, \mathrm{Wu} 04\right]\right)$. Na Seção 1.5 apresentamos dois casos particulares do OCT que generalizam o MRCT introduzidos por Wu et al. no ano 2000 ([WCT00a]). Na Seção 1.6, última deste capítulo, apresentamos uma relação do OCT e suas principais variantes NP-difíceis estudadas na literatura. No Capítulo 2 introduzimos novas variantes do OCT, onde algumas delas generalizam problemas anteriormente estudados. Provamos que todos os problemas introduzidos são NP-difíceis em grafos gerais e métricos. No Capítulo 3 continuamos a revisão dos resultados da literatura, desta vez analisando os diferentes algoritmos de aproximação conhecidos anteriormente. No Capítulo 4 apresentamos um lema que dá condições suficientes para que algumas variantes do OCT métrico admitam PTAS, e mostramos como aplicar este resultados para diferentes problemas. Nos Capítulos 5 e 6 mostramos um outro PTAS, desta vez para um caso particular do OCT que generaliza os PTASs obtidos em trabalhos anteriores para versões particulares do OCT. Finalmente, no último capítulo mostramos as principais conclusões deste trabalho e discutimos sobre possíveis linhas de pesquisa no futuro.

Antes de continuarmos, é importante mencionar que, a menos que seja especificado, todos os grafos neste trabalho são simples, conexos e não-dirigidos. Além disso, com o objetivo de simplificar a notação e algumas fórmulas, dado um grafo $G$, denotamos o conjunto de vértices de $G$ por $V_{G}$ com cardinalidade $n_{G}=\left|V_{G}\right|$ e o conjunto de arestas por $E_{G}$. Quando o grafo $G$ estiver implícito pelo contexto serão usados $V$ ou $E$ ao invés de $V_{G}$ ou $E_{G}$, e $n=n_{G}$.

\section{$1.1 \quad$ OCT}

O OCT foi introduzido no ano 1974 por Hu em [Hu74]. Antes de definir o problema, precisamos apresentar a definição de distância entre vértices:

Definição 1.1. Dado um grafo $G$ com comprimentos não-negativos associados às arestas, o com-

primento de um caminho em $G$ é dado pela soma dos comprimentos das suas arestas (um 
caminho sem arestas tem comprimento zero); sendo $d(H, x, y)$ a distância entre dois vértices $x e$ y em um sub-grafo $H$ de $G$, que é igual ao comprimento de um caminho de comprimento mínimo entre $x$ e y em $H$.

Agora, definimos formalmente o OCT:

Problema 1.2. OCT - Optimum Communication spanning Tree problem.

Entrada: Um grafo $G$ com uma função não negativa de comprimento sobre as arestas, $\omega: E \rightarrow$ $\mathbb{Q}_{+}$, e uma função não negativa de requerimento entre todo par de vértices, $\psi: V \times V \rightarrow \mathbb{Q}_{+}$.

Saída: Uma árvore geradora $T$ de $G$ que minimize o custo total de comunicação:

$$
C(T)=\sum_{u \in V} \sum_{v \in V} \psi(u, v) d(T, u, v) .
$$

No mesmo artigo em que definiu o OCT, Hu apresentou dois casos particulares deste problema para os quais deu soluções polinomiais. Estes são o ORT (Optimum Requeriment spanning Tree problem) e um caso particular do MRCT métrico ${ }^{1}$ (Minimium Routing Cost spanning Tree problem), que são discutidos a seguir.

\subsubsection{ORT}

O ORT é o caso particular de OCT em que o grafo $G$ é completo e todas as arestas têm comprimento unitário (isto é, $\omega: E \rightarrow 1$ ).

A proposta de Hu para solucionar o ORT mantém o mesmo grafo $G$ e define uma função de capacidade para as arestas $\chi: E \rightarrow \mathbb{Q}_{+}$, onde a aresta $e$ que une os vértices $u$ e $v$ recebe como capacidade $\chi_{e}=\psi(u, v)+\psi(v, u)$. O objetivo é, então, encontrar uma árvore de corte, uma árvore $T^{*}$ geradora do grafo tal que:

Se cada aresta e $\in T^{*}$ tem associado o valor $\beta_{e}$ que é igual a capacidade do corte $\left(V_{X}, V_{\bar{X}}\right)$ em $G$, onde $X$ e $\bar{X}$ são as componentes resultando da remoção de e em $T^{*}$, e a capacidade do corte é a soma das capacidades das arestas com uma ponta em $X$ e a outra em $\bar{X}$. Então, o fluxo máximo entre qualquer par de vértices $u$ e $v$ em $G$ tem valor: $f(u, v)=\min \left\{\beta_{e}\right\}_{e \in E_{P_{u v}}}$, onde $P_{u v}$ é o caminho entre u e $v$ em $T^{*}$.

O problema de encontrar a árvore de corte foi solucionado em um artigo anterior de Gomory e Hu, [GH61]. A proposta nele é solucionar $n-1$ problemas de fluxo máximo, cada um deles com tempo de execução $O\left(n^{3}\right)$ ([FF57]), resultando em um custo geral de $O\left(n^{4}\right)$.

Para demonstrar que a árvore de corte acima é uma solução ótima para ORT, observamos que cada árvore geradora $T$ de $G$ dá origem a $n-1$ cortes que não se cruzam. Dizemos que dois cortes $(X, \bar{X})$ e $(Y, \bar{Y})$ se cruzam se e somente se $X \cap Y \neq \emptyset, X \cap \bar{Y} \neq \emptyset, \bar{X} \cap Y \neq \emptyset$ e $\bar{X} \cap \bar{Y} \neq \emptyset$. A Figura 1.3 mostra um exemplo de dois cortes que se cruzam.

Considere as arestas $e_{1}, e_{2} \in E_{T}$, e observe que a remoção de $e_{1}$ divide a árvore em duas componentes, que são também árvores, $T_{e_{1}}$ e $\bar{T}_{e_{1}}$, definindo o corte $c_{1}=\left(V_{T_{e_{1}}}, V_{\bar{T}_{e_{1}}}\right)$. Sem perda de generalidade suponha que $e_{2} \in E_{T_{e_{1}}}$, logo a remoção de $e_{2}$ divide $T_{e_{1}}$ em duas componentes $T_{e_{12}}$ e $\bar{T}_{e_{12}}$. Sem perda de generalidade considere que $e_{1}$ conecta as componentes $\bar{T}_{e_{12}}$ e $\bar{T}_{e_{1}}$. Logo, a remoção de $e_{2}$ define em $T$ o corte $c_{2}=\left(V_{T_{e_{12}}}, V_{\bar{T}_{e_{12}}} \cup V_{\bar{T}_{e_{1}}}\right)$. Como $T_{e_{12}} \cap \bar{T}_{e_{2}}=\emptyset$, temos que $c_{1}$

\footnotetext{
${ }^{1}$ O MRCT métrico foi denominado por Hu em seu artigo como ODT (Optimum Distance spanning Tree problem), porém na literatura o problema é mais conhecido por MRCT.
} 


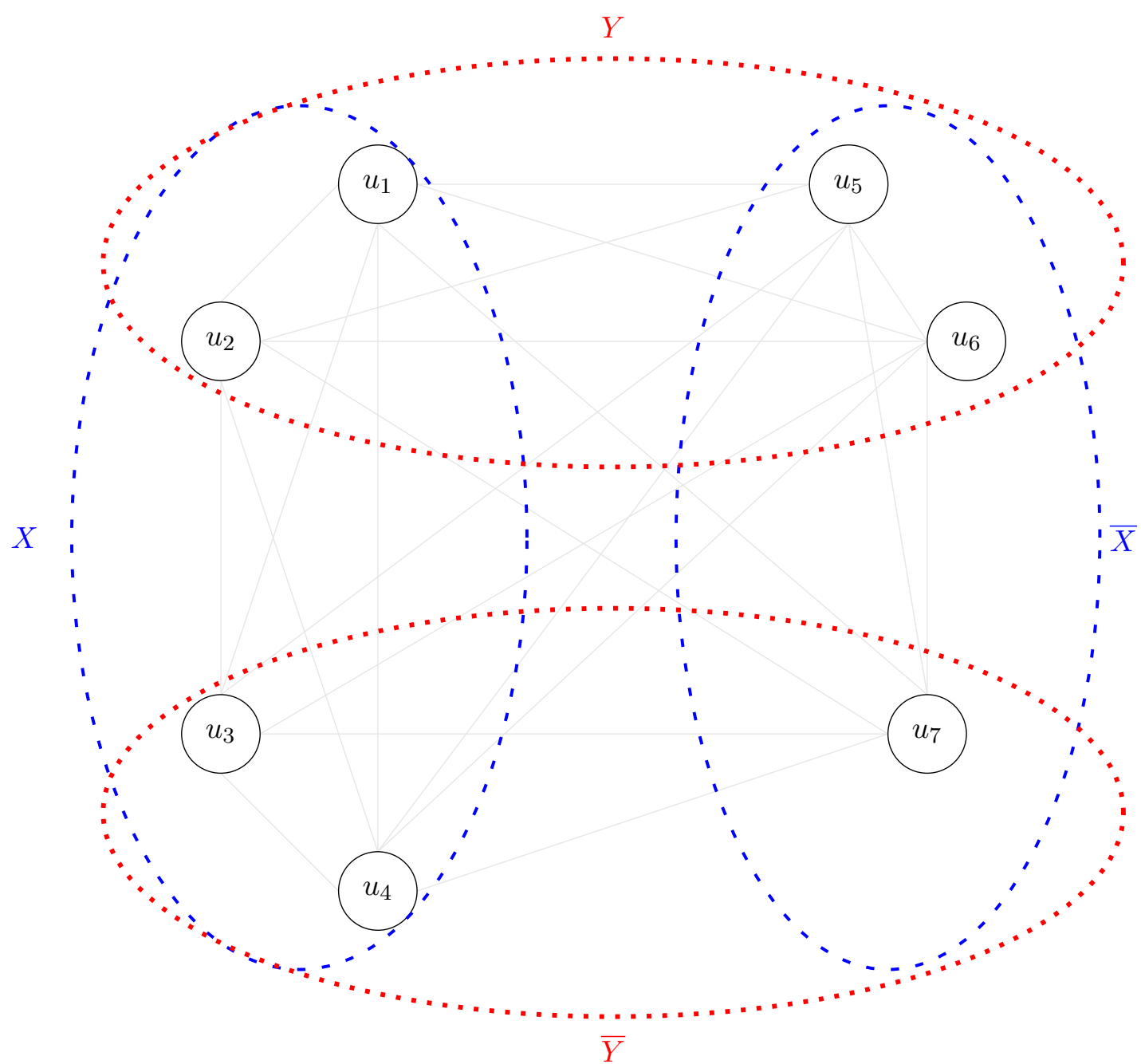

Figura 1.3: Figura que ilustra dois cortes que se cruzam, estes são o corte $\left(X=\left\{u_{1}, u_{2}, u_{3}, u_{4}\right\}, \bar{X}=\right.$ $\left.\left\{u_{5}, u_{6}, u_{7}\right\}\right)$ (em linhas descontinuas) e o corte $\left(Y=\left\{u_{1}, u_{2}, u_{5}, u_{6}\right\}, \bar{Y}=\left\{u_{3}, u_{4}, u_{7}\right\}\right)$ (em pontos).

e $c_{2}$ não se cruzam, concluindo que cortes definidos a partir de remoção de arestas não se cruzam. A Figura 1.4 ilustra esta análise.

Assim, o conjunto dos cortes definidos a partir da remoção de cada aresta de $T$ resulta em um conjunto de $n-1$ cortes que não se cruzam. Como o comprimento de todas as arestas é unitário, o custo de comunicação de $T$ pode ser calculado somando o total de requerimento que passa por cada aresta. O total de requerimento que passa por $e \in E_{T}$ é a soma dos requerimentos entre todo par de vértices conectados em $T$ por um caminho que contém $e$, ou seja $\beta_{e}$, a capacidade do corte $\left(V_{T_{e}}, V_{\bar{T}_{e}}\right)$. Logo, o custo total de comunicação de $T$ é igual à soma das capacidades dos $n-1$ cortes associados às arestas de $T$.

De um resultado prévio obtido por Adolphson e Hu ([AH73]) se deriva que a soma das capacidades dos $n-1$ cortes associados à árvore de corte $T^{*}$ é menor ou igual que a soma das capacidades de qualquer outro conjunto de $n-1$ cortes que não se cruzem, o que implica que a árvore de corte é uma árvore geradora de comunicação mínima para o ORT. 


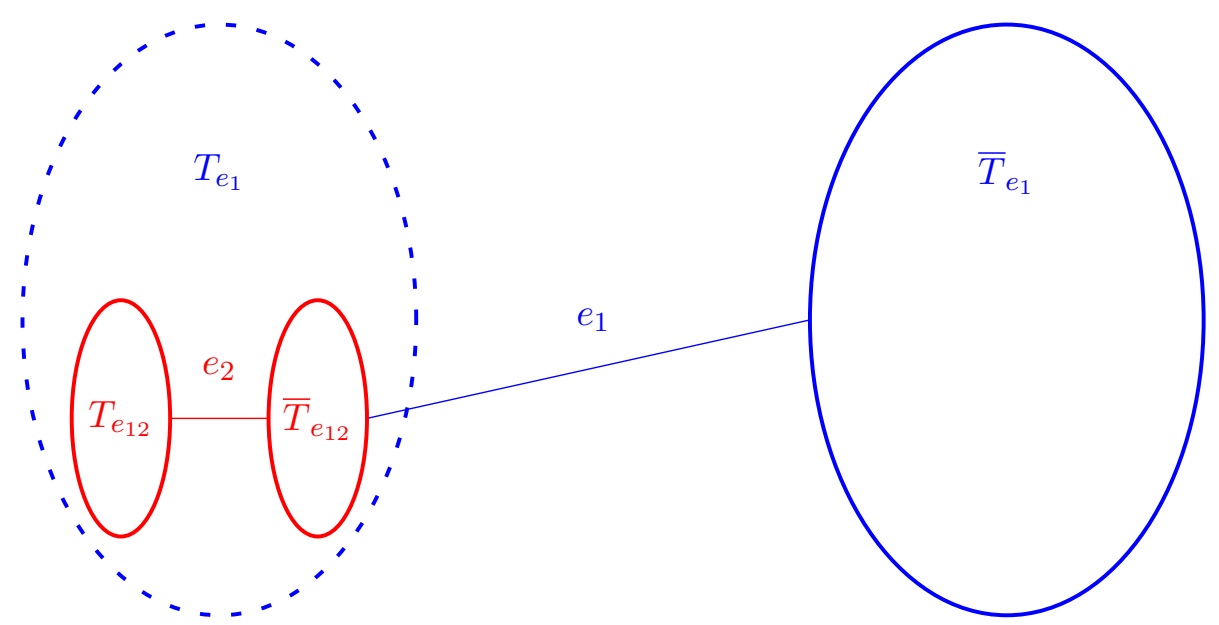

Figura 1.4: Figura que mostra dois cortes $\left(V_{T_{e_{1}}}, V_{\bar{T}_{e_{1}}}\right)$ e $\left(V_{T_{e_{12}}}, V_{\bar{T}_{e_{12}}} \cup V_{\bar{T}_{e_{1}}}\right)$ definidos a partir da remoção das arestas $e_{1}$ e $e_{2}$ respectivamente. Note que $T_{e_{12}} \cap \bar{T}_{e_{1}}=\emptyset$, o que implica que estes cortes não se cruzam.

\subsubsection{Um caso particular do MRCT métrico}

O MRCT é o caso particular do OCT em que o requerimento entre todo par de vértices é unitário, isto é, para todo $u, v \in V, \psi(u, v)=\psi(v, u)=1$. Formalmente:

Problema 1.3. MRCT - Minimum Routing Cost spanning Tree problem.

Entrada: Um grafo $G$ com uma função não negativa de comprimento sobre as arestas, $\omega: E \rightarrow$ $\mathbb{Q}_{+}$.

Saída: Uma árvore $T$ geradora de $G$ que minimize o custo roteamento (custo total de comunicação):

$$
C(T)=\sum_{u \in V} \sum_{v \in V} d(T, u, v)
$$

O MRCT métrico é o caso particular em que o grafo $G$ é métrico, isto é, $G$ é completo e a função de comprimento nas arestas satisfaz a desigualdade triangular, ou seja para todo $u, v, w \in V$ $\omega(u v)+\omega(u w) \geq \omega(v w)$.

A proposta de Hu não foi solucionar o MRCT métrico, mas sim dar condições suficientes para que uma estrela fosse uma solução ótima. Neste caso bastaria escolher uma estrela geradora de comunicação mínima entre as $n-1$ possíveis estrelas geradoras de $G$.

A condição encontrada foi:

Condição 1.4. Para quaisquer $u, v, w \in V \operatorname{com} \omega(u v) \leq \omega(u w) \leq \omega(v w)$ existe uma constante positiva $t \leq \frac{n-2}{2 n-2}$ tal que $\omega(u v)+t \omega(u w) \geq \omega(v w)$.

Se a condição acima é satisfeita, então existe uma estrela que é solução ótima para o problema. De fato, se tomarmos uma árvore geradora com pelo menos dois vértices internos podemos aumentar seu número de folhas sem aumentar o custo de comunicação. Para isto é necessário introduzir a definição de vértice interno extremal de uma árvore. Este é um vértice interno da árvore que tem no máximo um vizinho interno, sendo o resto dos seus vizinhos folhas da árvore.

Considere uma árvore geradora $T$ de $G$ com pelo menos dois vértices internos. Seja $u \in V$ um vértice interno extremal de $T$ com não mais de $\frac{n}{2}$ vizinhos em $T$. Note que tal vértice existe pois 
a árvore tem no mínimo dois vértices internos extremais e se um tem mais do que $\frac{n}{2}$ vizinhos pelo menos $\frac{n}{2}$ são folhas o que implica que o outro deve ter no máximo $n-1-\frac{n}{2}>\frac{n}{2}$ vizinhos. Tome $v$ o vértice interno de $T$ vizinho de $u$. Defina, então, a árvore geradora $T^{\prime}$ de $G$ obtida após remover de $T$ as arestas entre $u$ e as folhas vizinhas dele e colocar essas folhas como adjacentes de $v$. Com isto, $u$ passa a ser uma folha de $T^{\prime}$ o que faz com que o número de vértices internos em $T^{\prime}$ seja um a menos que em $T$. Se $u$ tem $k$ folhas vizinhas em $T$, dadas pelo conjunto $\left\{w_{i}\right\}_{i=1}^{k}$, a diferença dos custos de comunicação entre $T$ e $T^{\prime}$ é dada por:

$$
\begin{aligned}
C(T)-C\left(T^{\prime}\right)= & (k+1)(n-k-1) \omega(u v)+(n-1) \sum_{i=1}^{k} \omega\left(u w_{i}\right) \\
& -(n-1) \omega(u v)-(n-1) \sum_{i=1}^{k} \omega\left(v w_{i}\right) \\
= & k(n-k-2) \omega(u v)+(n-1) \sum_{i=1}^{k}\left(\omega\left(u w_{i}\right)-\omega\left(v w_{i}\right)\right) \\
= & (n-1) \sum_{i=1}^{k}\left(\frac{(n-k-2)}{(n-1)} \omega(u v)+\omega\left(u w_{i}\right)-\omega\left(v w_{i}\right)\right) .
\end{aligned}
$$

Como $k \leq \frac{n}{2}-1$ :

$$
\begin{aligned}
C(T)-C\left(T^{\prime}\right) & \geq(n-1) \sum_{i=1}^{k}\left(\frac{(n-2)}{(2 n-2)} \omega(u v)+\omega\left(u w_{i}\right)-\omega\left(v w_{i}\right)\right) \\
& \geq\left((n-1) \sum_{i=1}^{k} t \omega(u v)+\omega\left(u w_{i}\right)-\omega\left(v w_{i}\right)\right) .
\end{aligned}
$$

Se a condição 1.4 for verdadeira, então é fácil ver que para cada $i \in\{1, \cdots, k\}$ se satisfaz $t \omega(u v)+\omega\left(u w_{i}\right)-\omega\left(v w_{i}\right) \geq 0$, o que implica que $C(T)-C\left(T^{\prime}\right) \geq 0$, garantindo que o processo pode ser repetido até obter uma estrela com custo de comunicação menor ou igual que o de $T$. Desta forma, para uma árvore ótima, se não for uma estrela, é possível obter uma estrela $T^{*}$ que também é uma solução ótima. Assim, se a condição 1.4 é satisfeita, sempre existe uma árvore geradora de comunicação mínima que é uma estrela para MRCT métrico.

\subsection{Primeira prova de complexidade}

Em [JLRK78] foi provado que OCT é NP-difícil. A demonstração basou-se em uma redução da 3-EC (que definimos adiante e foi provado ser NP-completo por [GJ79]) para o MRCT (introduzido na seção anterior). A seguir damos uma versão da prova apresentada pelos autores.

Para isto, primeiro definimos o 3-EC:

Problema 1.5. 3-EC - 3-Exact Cover.

Entrada: Um conjunto de $n$ elementos $T=\left\{t_{1}, \cdots, t_{n}\right\}$ e um conjunto $S=\left\{s_{1}, \cdots, s_{m}\right\}$ de $m$ subconjuntos de $T$, cada um com exatamente 3 elementos. 
Saída: Existe um subconjunto $S^{*}$ de $S$ tal que os elementos de $S^{*}$ são disjuntos dois a dois e a união dos elementos de $S^{*}$ é igual a $T$.

Agora, passamos a provar o seguinte resultado.

Teorema 1.6. $O$ MRCT é NP-difícil e, a menos que $P=N P$, este problema não admite FPTAS. ([JLRK'78])

Demonstração. Considere uma instância $I=\langle T, S\rangle$ de 3-EC e um inteiro $M$ suficientemente grande (para simplificar a demonstração o valor de $M$ será definido no final da mesma). Definimos o grafo $G=(V, E)$ da seguinte forma (a Figura 1.5 mostra este grafo):

- Para cada $t \in T$ adicione um vértice $u_{t}$ em $V$.

- Para cada $s \in S$ adicione um vértice $u_{s}$ em $V$.

- Adicione também um vértice central $u_{0}$ e outros $M$ vértices $\left(\left\{u_{1}, \cdots, u_{M}\right\}\right)$ em $V$.

- Se um elemento $t \in T$ está contido em um conjunto $s \in S$ então adicione a aresta $\left(u_{t}, v_{s}\right)$ em $E$.

- Para cada $s \in S$ adicione a aresta $\left(u_{s}, u_{0}\right)$ em $E$.

- Finalmente, para cada $i \in\{1, \cdots, M\}$ adicione a aresta $\left(u_{0}, u_{i}\right)$ em $E$.

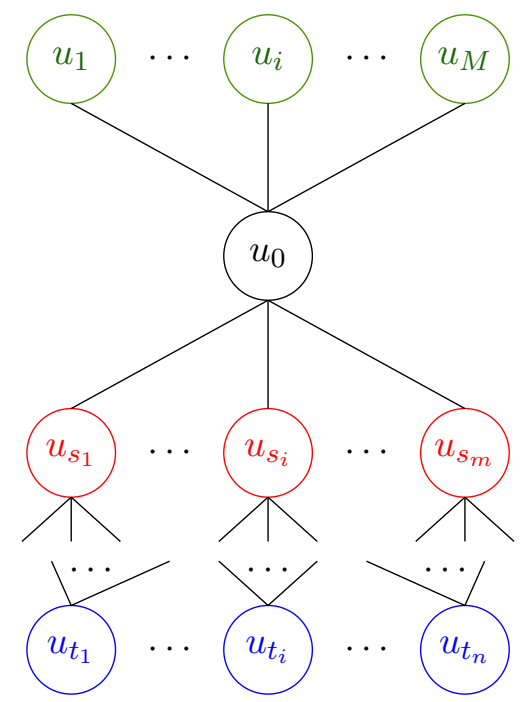

Figura 1.5: Grafo construído a partir da instância I de 3-EC. Observe que qualquer árvore geradora dele deve conter todas as arestas $\left(u_{i}, u_{0}\right)$ para $i \in\{1, \cdots, M\}$.

Logo, atribuindo 1 como comprimento de cada aresta de $G$, obtemos a instância do MRCT $I^{\prime}=\langle G, \omega: E \rightarrow 1\rangle$ associada à instância $I$ de 3-EC.

Como toda árvore geradora de $G$ deve conter as arestas $\left(u_{i}, u_{0}\right)$ para $i \in\{1, \cdots, M\}$ e consideramos que $M$ é um valor inteiro suficientemente grande, então qualquer solução ótima de MRCT da instância $I^{\prime}$ deve garantir que a distância de todo vértice para $u_{0}$ seja mínima. Isto é, que os vértices $u_{s}(s \in S)$ sejam adjacentes a $u_{0}$ em tal solução e os vértices $u_{t}(t \in T)$ folhas adjacentes a algum $u_{s}$. Denote como boa qualquer solução que tenha esta propriedade (que a distância de qualquer vértice para $u_{0}$ nela seja mínima). 
Note que em toda solução boa o custo de comunicação de $u_{0}$ para o resto do grafo é igual, o mesmo acontece com o custo de comunicação de cada $u_{i}$ com $i \in\{1, \cdots, M\}$ para o resto do grafo e com o custo de comunicação entre todo par de vértices $u_{s_{1}}$ e $u_{s_{2}} \operatorname{com} s_{1}, s_{2} \in S$. Logo, a única diferença de custo entre duas soluções boas quaisquer pode estar no custo de comunicação de vértices $u_{t_{1}}$ para vértices $u_{t_{2}}$ ou vértices $u_{s}$ com $t_{1}, t_{2} \in T$ e $s \in S$.

Como todo vértice $u_{t} \operatorname{com} t \in T$ em uma solução boa é uma folha adjacente a algum vértice $u_{s} \operatorname{com} s \in S$, então o custo de comunicação de cada $u_{t}$ para o conjunto de vértices $\left\{u_{s}\right\}_{s \in S}$ é o mesmo em toda solução boa (pois a distância é 1 para o vértice ao qual é adjacente e 3 para o resto). Portanto, se duas soluções boas têm custos de comunicação diferentes, a diferença é dada pelos custos de comunicação entre vértices do conjunto $\left\{u_{t}\right\}_{t \in T}$.

Em uma solução boa a distância entre dois vértices $u_{t_{1}}$ e $u_{t_{2}}$ é 2 se são adjacentes ao mesmo $u_{s}$ e 4 caso contrário $\left(t_{1}, t_{2} \in T\right.$ e $\left.s \in S\right)$. Então, denote por irmãos o par de vértices $u_{t_{1}}$ e $u_{t_{2}}$ se são adjacentes do mesmo $u_{s}$ em uma solução boa, caso contrário denote-os por primos. É fácil ver que uma solução boa é ótima se maximiza o número de irmãos. Como o número máximo de elementos em $\left\{u_{t}\right\}_{t \in T}$ que podem ser adjacentes de um mesmo $u_{s}(s \in S)$ é 3, então o máximo número de irmãos adjacentes a um mesmo $u_{s}$ é também 3 e qualquer solução boa tem no máximo $\frac{n(n-1)}{6}$ irmãos.

Observe que se uma solução boa $X^{\prime}$ tem $\frac{n(n-1)}{6}$ irmãos então, fazendo:

$$
X=\left\{s \in S: u_{s} \text { é adjacente a três irmãos em } X^{\prime}\right\},
$$

temos que $X \subseteq S$ e $X$ é uma 3-cobertura exata de $T$, portanto 3-EC para $I$ é verdadeira. Analogamente, se 3-EC é verdadeira para $I$, então existe uma 3-cobertura exata $X \subseteq S$ de $T$ tal que, se para cada $t \in T, s_{t}$ é o elemento em $X$ que contém $t$, então a solução boa $X^{\prime}$ de $I^{\prime}$ em que cada $u_{t}$ é colocado como adjacente de $u_{s t}$ tem $\frac{n(n-1)}{6}$ irmãos. Logo, 3-EC é verdadeira com instância $I$ se e somente se existe uma solução boa com $\frac{n(n-1)}{6}$ irmãos para MRCT com instância $I^{\prime}$.

O custo de comunicação de uma solução boa com $\frac{n(n-1)}{6}$ irmãos pode ser calculado por partes, como segue:

- Para cada $u_{i}$ com $1 \leq i \leq M$, o custo de comunicação é igual a $2(M-1)+1+2 m+3 n$ :

- 2 para cada vértice $u_{j}$ com $1 \leq j \leq M$ e $i \neq j$;

- 1 para o vértice $u_{0}$;

- 2 para cada vértice $u_{s}$ com $s \in S$;

- 3 para cada vértice $u_{t}$ com $t \in T$.

- Para $u_{0}$ o custo de comunicação tem valor $M+m+2 n$ :

- 1 para cada vértice $u_{i}$ com $1 \leq i \leq M$;

- 1 para cada vértice $u_{s}$ com $s \in S$;

- 2 para cada vértice $u_{t}$ com $t \in T$.

- Para cada $u_{s_{1}}$ com $s_{1} \in S$, o custo de comunicação é igual a $2 M+1+2(m-1)+3\left(n-n_{s}\right)+n_{s}$, sendo $n_{s}$ o número de vértices adjacentes na solução de $\left\{u_{t}\right\}_{t \in T}$ :

- 2 para cada vértice $u_{j}$ com $1 \leq j \leq M$; 
- 1 para o vértice $u_{0}$;

- 2 para cada vértice $u_{s_{2}}$ com $s_{2} \in S$;

- 3 para cada vértice $u_{t}$ que não e adjacente a ele $(t \in T)$;

- 1 para cada vértice $u_{t}$ adjacente a ele $(t \in T)$.

- Para cada $u_{t_{1}}$ com $t_{1} \in T$, o custo de comunicação é igual a $3 M+2+1+3(m-1)+4+4(n-3)$ :

- 3 para cada vértice $u_{j}$ com $1 \leq j \leq M$;

- 2 para o vértice $u_{0}$;

- 1 para o vértice $u_{s}$ adjacente a ele;

- 3 para cada vértice $u_{s}$ com $s \in S$ que não é adjacente a ele;

- 2 para cada um dos dois vértices irmãos em $\left\{u_{t}\right\}_{t \in T-t_{1}}$;

- 4 para cada um dos $n-3$ vértices primos em $\left\{u_{t}\right\}_{t \in T-t_{1}}$.

Logo, o custo de uma solução boa $X \operatorname{com} \frac{n(n-1)}{6}$ irmãos é:

$$
\begin{aligned}
C(X)= & \sum_{i=1}^{M}(2(M-1)+1+2 m+3 n)+M+m+2 n \\
& +\sum_{s \in S}\left(2 M+1+2(m-1)+3\left(n-n_{s}\right)+n_{s}\right) \\
& +\sum_{t \in T} 3 M+2+1+3(m-1)+4+4(n-3) \\
= & 2 M^{2}+2 m^{2}+4 n^{2}+4 m M+6 n M+5 m n-3 n-2 m \sum_{s \in S} n_{s} \\
= & 2 M^{2}+2 m^{2}+4 n^{2}+4 m M+6 n M+5 m n-3 n-2 m n \\
= & 2 M^{2}+2 m^{2}+4 n^{2}+4 m M+6 n M+3 m n-3 n
\end{aligned}
$$

Não é difícil ver que um valor de $M=6(m n)^{2}$ é maior que o custo total de comunicação entre os vértices $u_{0}, u_{s}(s \in S)$ e $u_{t}(t \in T)$, implicando que é grande o suficiente para forçar que somente soluções boas sejam ótimas.

Como o custo de uma solução boa com $\frac{n(n-1)}{6}-r$ irmãos é $2 M^{2}+2 m^{2}+4 n^{2}+4 m M+6 n M+$ $3 m n-3 n+4 r(r \geq 0)$. Temos que se definimos $K=2 M^{2}+2 m^{2}+4 n^{2}+4 m M+6 n M+3 m n-3 n$, somente soluções boas com $\frac{n(n-1)}{6}$ irmãos terão custo de comunicação $K$.

Portanto, o problema de decisão associado ao MRCT com instância $I^{\prime}$ e inteiro $K$, que decide se existe uma solução para $I^{\prime}$ com custo menor ou igual que $K$, responde verdadeiro se e somente se a solução ótima de $I^{\prime}$ é boa e tem $\frac{n(n-1)}{6}$ irmãos o que implica que responde verdadeiro se e somente se 3-EC com instância $I$ responde verdadeiro.

É fácil ver que o problema de decisão associado a OCT que recebe uma instância $I=\langle G, \omega, \phi\rangle$ e um inteiro $K$, e decide se existe uma árvore geradora de $G$ com custo menor ou igual que $K$ pertence à classe NP. Para isto, é suficiente considerar o caso em que a resposta é afirmativa, sendo o certificado uma árvore $T$ geradora de $G$ com $C(T) \leq K$. Verificar que $T$ é geradora de $G$ e 
calcular o custo de $T$, são operações que podem ser feitas em tempo polinomial no tamanho de $I$. Este raciocínio é válido para qualquer caso particular do OCT, como é o caso do MRCT.

Logo, como a redução do 3-EC para o MRCT é polinomial temos que MRCT e o caso mais geral OCT são NP-difíceis.

Observe que na redução usada, as instâncias obtidas para o MRCT garantem que todos os parâmetros numéricos estão limitados por um polinômio no tamanho do grafo (número de vértices, $\left.n_{G}\right)$. Note que, tanto os requerimentos quanto o comprimentos das arestas tem valor 1 , fazendo que o custo de comunicação de qualquer árvore geradora seja $O\left(n_{G}^{4}\right)$. Isto implica que o problema de decisão associado ao MRCT seja fortemente NP-completo, logo, o MRCT não admite FPTAS a menos que $\mathrm{P}=\mathrm{NP}$.

\subsection{Redução do MRCT ao caso métrico}

Como visto na seção anterior já em 1978 se havia demonstrado que o OCT e particularmente o MRCT são NP-difíceis, porém se passaram duas décadas até o primeiro resultado sobre complexidade dos casos métricos destes problemas.

Assim em 1998, Wu et al. ([WLB ${ }^{+}$98]), apresentam uma redução do MRCT ao MRCT métrico, provando que este último é também NP-difícil e consequentemente o OCT métrico também.

Teorema 1.7. O MRCT métrico é NP-difícil e, a menos que $P=N P$, este problema não admite FPTAS. ([WL $B^{+}$98])

Demonstração. Para isso, considere uma instância $I=\langle G, \omega\rangle$ do MRCT e construa a instância $\bar{I}=\langle\bar{G}, \bar{\omega}\rangle$ do MRCT métrico que se obtém fazendo o fecho métrico de $I$, ou seja $\bar{G}$ é o grafo completo com o mesmo conjunto de vértices de $G$, em que $\bar{\omega}(u, v)=d(G, u, v)$.

Suponha que $\bar{T}$ é uma solução do MRCT métrico com instância $\bar{I}$, e denotemos por arestas ruins aquelas arestas de $\bar{T}$ que não pertencem a $G$ ou que têm um comprimento associado diferente em $I$ e $\bar{I}$ (isto é, uma aresta $e$ de $\bar{T}$ é ruim se $e \notin V_{G}$ ou se $\omega(e) \neq \bar{\omega}(e)$ ). A ideia é substituir as arestas ruins (se existirem) sem piorar o custo da nova árvore até obter uma árvore $T$ que é solução do MRCT com instância $I$ e cujo custo de comunicação é menor ou igual que o de $\bar{T}$.

Observe que se $\bar{T}$ não possui arestas ruins, então ela própria é solução do MRCT com instância $I$.

Caso contrário $\bar{T}$ tem pelo menos uma aresta ruim. Denotemos por $(u, v)$ uma aresta ruim de $\bar{T}$ e suponhamos que um caminho mínimo em $G$ entre $u$ e $v$ é $P=u, w, \cdots, v$.

Se enraizamos a árvore $\bar{T}$ no vértice $u$ e denotamos por $x$ o pai de $w$ neste enraizamento, então temos as seguintes possibilidades (ambas ilustradas pela Figura 1.6):

- Se $v$ não é ancestral de $w$ em $\bar{T}$ enraizada no vértice $u$, definimos a árvore $T_{1}$ como o resultado de trocar a aresta $(u, v)$ por $(w, v)$ em $\bar{T}$ e a árvore $T_{2}$ trocando em $T_{1}$ a aresta $(x, w)$ por $(u, w)$. Isto é:

$$
\begin{aligned}
& T_{1}=\bar{T} \cup(w, v) \backslash(u, v) \\
& T_{2}=T_{1} \cup(u, w) \backslash(x, w) .
\end{aligned}
$$


- Caso contrário, a árvore $T_{1}$ resulta de trocar a aresta $(u, v)$ por $(u, w)$ em $\bar{T}$, enquanto $T_{2}$ é obtida trocando a aresta $(x, w)$ por $(w, v)$ em $T_{1}$. Isto é:

$$
\begin{aligned}
& T_{1}=\bar{T} \cup(u, w) \backslash(u, v) \\
& T_{2}=T_{1} \cup(w, v) \backslash(x, w) .
\end{aligned}
$$

Finalmente, seja qual for o caso, definimos uma nova árvore $T^{\prime}$ como $T_{1}$ se $C\left(T_{1}\right) \leq C\left(T_{2}\right)$, caso contrário $T^{\prime}=T_{2}$. Com isso, repetimos o processo com $T^{\prime}$ até obtermos uma árvore sem arestas ruins. Observe que neste processo toda aresta ruim é substituída por duas novas arestas, onde uma delas pode também ser ruim, mas como veremos adiante o algoritmo garante obter uma árvore sem arestas ruins.

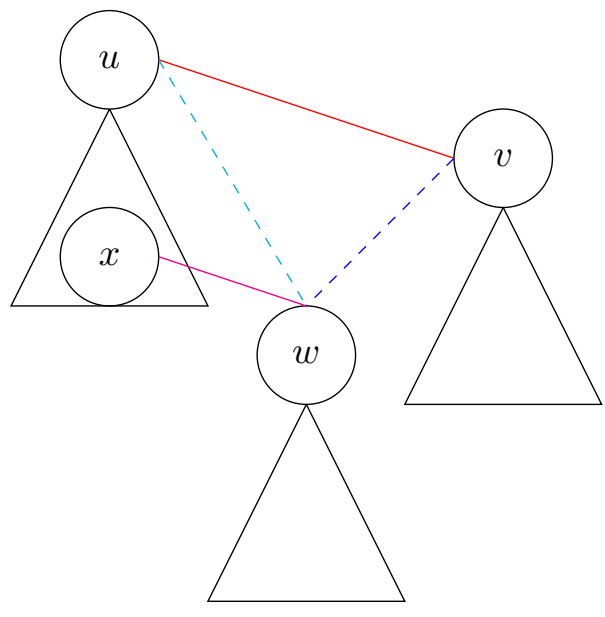

(a)

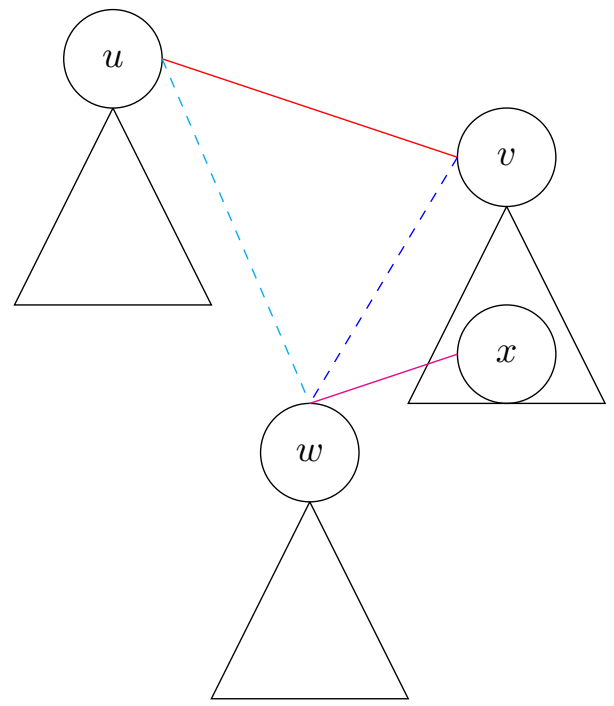

(b)

Figura 1.6: Figura que ilustra os dois casos possiveis ao tentar remover a aresta ruim $(u, v)$ da árvore enraizada em u. Na esquerda, opção (a), ilustramos o caso em que $v$ não é ancestral de $w$, onde para obter $T_{1}$ trocamos a aresta $(u, v)$ pela $(w, v)$ e para obter $T_{2}$ trocamos também a aresta $(x, w)$ pela $(u, w) . N a$ direita, opção $(b)$, ilustramos o caso em que $v$ é ancestral de $w$, para obter $T_{1}$ trocamos a aresta $(u, v)$ por $(u, w)$ e para obter $T_{2}$ trocamos a aresta $(x, w)$ pela $(v, w)$. Note que, se no caso $(b)$ enraizamos a árvore em $v$ ao invés de $u$, então temos exatamente o mesmo caso que a opção (a) ilustra.

Vamos agora analisar o custo computacional do processo descrito acima. É um resultado bem conhecido que podemos obter um conjunto de caminhos mínimos entre todo par de vértices de $G$ em tempo $O\left(n^{3}\right)$. Perceba que podemos obter esse conjunto de caminhos de forma tal que satisfaça a seguinte propriedade:

Se $P=u, w, \cdots, v$ e $Q=w, \cdots, v$ são os caminhos mínimos obtidos entre $u e v, e, w e v$, respectivamente, então $P=(u, w) \cup Q$.

Ou seja, se $P$ é um caminho mínimo que calculamos, então o caminho mínimo entre qualquer par de vértices em $P$ deve ser um sub-caminho de $P$.

Observe que cada vez que eliminamos uma aresta ruim $(u, v)$, inserimos duas arestas, uma boa (que não é ruim) e outra que pode ser ou não ruim; onde a aresta boa $e$, é um extremo de $P$, o caminho mínimo precalculado entre $u$ e $v$, enquanto a outra é a aresta que conecta os vértices extremos de $P \backslash e$ com comprimento $\omega(P \backslash e)$. Como um caminho de $G$ tem no máximo $n-1$ arestas, temos que para eliminar uma aresta ruim $(u, v)$ de $\bar{T}$, no máximo serão substituídas $n-1$ arestas 
ruins em todo o processo incluindo a própria $(u, v)$; e como $\bar{T}$ é uma árvore então tem no máximo $n-1$ arestas, o que implica que o número total de iterações para obter uma árvore sem arestas ruins é $O\left(n^{2}\right)$, onde cada iteração pode ser feita em tempo $O(n)$ o que inclui enraizar a árvore, percorrer a mesma e trocar as arestas. Assim o processo todo pode ser feito em tempo $O\left(n^{3}\right)$.

Agora vamos analisar o custo da árvore $T$ obtida no final do processo de eliminação das arestas ruins de $\bar{T}$. Para isso, analisaremos o custo da árvore $T^{\prime}$, que é obtida após eliminar a aresta ruim $(u, v)$ de $\bar{T}$.

Lembre que $\bar{T}$ é enraizada em $u$. Analisaremos o caso em que $v$ não é ancestral de $w$ (se ao invés de enraizarmos a árvore em $u$, a enraizamos em $v$, então a análise do caso em que $v$ é ancestral de $w$ é idêntica).

Se $C\left(T_{1}\right) \leq C(\bar{T})$, então temos que $C\left(T^{\prime}\right) \leq C\left(T_{1}\right) \leq C(\bar{T})$.

Caso contrário, $C\left(T_{1}\right)>C(\bar{T})$.

Se $i, j \in V_{G}$, denotemos por $\bar{T}_{i}$ a sub-árvore de $\bar{T}$ que tem como raiz o vértice $i$ e $\bar{T}_{i-j}$ a sub-árvore de $\bar{T}_{i}$ resultante de eliminar os elementos de $\bar{T}_{j}$ (isto é, $\bar{T}_{i-j}=\bar{T}_{i} \backslash \bar{T}_{j}$ ). Além disto, se $H_{1}, H_{2} \subseteq H$ então definimos o custo $C\left(H, H_{1}, H_{2}\right)=\sum_{i \in H_{1}} \sum_{j \in H_{2}} d(H, i, j)$ como o custo de roteamento entre os vértices em $H_{1}$ com os de $H_{2}$ em $H$.

Logo, como a distância entre dois vértices de $\bar{T}_{u-v}$ é a mesma em $\bar{T}$ e em $T_{1}$ e o mesmo acontece com a distância entre dois vértices em $\bar{T}_{v}$, temos que:

$$
\begin{aligned}
& C(\bar{T})<C\left(T_{1}\right) \\
\Rightarrow & C\left(\bar{T}, \bar{T}_{u-v}, \bar{T}_{v}\right)<C\left(T_{1}, \bar{T}_{u-v}, \bar{T}_{v}\right) \\
\Rightarrow & \left|V_{\bar{T}_{v}}\right| C\left(\bar{T}, u, \bar{T}_{u-v}\right)+\left|V_{\bar{T}_{v}}\right|\left|V_{\bar{T}_{u-v}}\right| \omega(u, v)<\left|V_{\bar{T}_{v}}\right| C\left(\bar{T}, w, \bar{T}_{u-v}\right)+\left|V_{\bar{T}_{v}}\right|\left|V_{\bar{T}_{u-v}}\right| \omega(w, v) \\
\Rightarrow & C\left(\bar{T}, u, \bar{T}_{u-v}\right)+\left|V_{\bar{T}_{u-v}}\right| \omega(u, v)<C\left(\bar{T}, w, \bar{T}_{u-v}\right)+\left|V_{\bar{T}_{u-v}}\right| \omega(w, v) \\
\Rightarrow & C\left(\bar{T}, u, \bar{T}_{u-v}\right)<C\left(\bar{T}, w, \bar{T}_{u-v}\right)-\left|V_{\bar{T}_{u-v}}\right|(\omega(u, v)-\omega(w, v)) \\
\Rightarrow & C\left(\bar{T}, u, \bar{T}_{u-v}\right)<C\left(\bar{T}, w, \bar{T}_{u-v}\right)-\left|V_{\bar{T}_{u-v}}\right| \omega(u, w) .
\end{aligned}
$$

Por outro lado:

$$
\begin{aligned}
C\left(T_{2}\right)-C(\bar{T})= & \left(C\left(T_{2}, \bar{T}_{u-v-w}, \bar{T}_{w}\right)-C\left(\bar{T}, \bar{T}_{u-v-w}, \bar{T}_{w}\right)\right) \\
& +\left(\boldsymbol{C}\left(\boldsymbol{T}_{\mathbf{2}}, \overline{\boldsymbol{T}}_{\boldsymbol{u}-\boldsymbol{v}}, \overline{\boldsymbol{T}}_{\boldsymbol{v}}\right)-\boldsymbol{C}\left(\overline{\boldsymbol{T}}_{\boldsymbol{\boldsymbol { T }}} \overline{\boldsymbol{T}}_{\boldsymbol{u}-\boldsymbol{v}}, \overline{\boldsymbol{T}}_{\boldsymbol{v}}\right)\right) .
\end{aligned}
$$

Observe que para todo par de vértices $i \in \bar{T}_{v}$ e $j \in \bar{T}_{u-v}$ a distância entre $i$ e $j$ em $T_{2}$ é menor ou igual que a distância entre eles em $\bar{T}$. Para isto, note que $\bar{T}_{u-v-w}$ está conectado a $\bar{T}_{v}$ em $\bar{T}$ pela aresta $(u, v)$ enquanto em $T_{2}$ estas sub-árvores estão conectadas pelas arestas $(u, w)$ e $(w, v)$ onde $\omega(u, v)=\omega(u, w)+\omega(w, v)$. Além disto $\bar{T}_{w}$ se conecta a $\bar{v}$ em $\bar{T}$ passando pelo vértice $u$ (isto é com uma distância maior do que $\omega(u, v))$ e em $T_{2}$ elas se conectam diretamente pela aresta $(v, w)$ cujo comprimento é $\omega(v, w)<\omega(u, v)$. Logo, temos que o segundo termo da equação acima é sempre menor ou igual ao zero, portanto: 


$$
\begin{aligned}
C\left(T_{2}\right)-C(\bar{T}) & \leq C\left(T_{2}, \bar{T}_{u-v-w}, \bar{T}_{w}\right)-C\left(\bar{T}, \bar{T}_{u-v-w}, \bar{T}_{w}\right) \\
& =\left|V_{\bar{T}_{w}}\right| C\left(\bar{T}, u, \bar{T}_{u-v-w}\right)+\left|V_{\bar{T}_{w}}\right|\left|V_{\bar{T}_{u-v-w}}\right| \omega(u, w)-\left|V_{\bar{T}_{w}}\right| C\left(\bar{T}, w, \bar{T}_{u-v-w}\right) \\
& =\left|V_{\bar{T}_{w}}\right|\left(C\left(\bar{T}, u, \bar{T}_{u-v-w}\right)+\left|V_{\bar{T}_{u-v-w}}\right| \omega(u, w)-C\left(\bar{T}, w, \bar{T}_{u-v-w}\right)\right) .
\end{aligned}
$$

Como para $i \in\{u, w\}$ se satisfaz que $C\left(\bar{T}, i, \bar{T}_{u-v-w}\right)=C\left(\bar{T}, i, \bar{T}_{u-v}\right)-C\left(\bar{T}, i, \bar{T}_{w}\right)$, temos que:

$$
\begin{aligned}
& C\left(\bar{T}, u, \bar{T}_{u-v-w}\right)-C\left(\bar{T}, w, \bar{T}_{u-v-w}\right) \\
= & C\left(\bar{T}, u, \bar{T}_{u-v}\right)-C\left(\bar{T}, u, \bar{T}_{w}\right)-\left(C\left(\bar{T}, w, \bar{T}_{u-v}\right)-C\left(\bar{T}, w, \bar{T}_{w}\right)\right) \\
= & \left(C\left(\bar{T}, u, \bar{T}_{u-v}\right)-C\left(\bar{T}, w, \bar{T}_{u-v}\right)\right)+\left(\boldsymbol{C}\left(\overline{\boldsymbol{T}}, \boldsymbol{w}, \overline{\boldsymbol{T}}_{\boldsymbol{w}}\right)-\boldsymbol{C}\left(\overline{\boldsymbol{T}}, \boldsymbol{u}, \overline{\boldsymbol{T}}_{\boldsymbol{w}}\right)\right) .
\end{aligned}
$$

Não é difícil perceber que o termo em negrito é sempre menor ou igual que zero. Logo, aplicando o resultado dado por 1.1 temos:

$$
\begin{aligned}
& C\left(\bar{T}, u, \bar{T}_{u-v-w}\right)-C\left(\bar{T}, w, \bar{T}_{u-v-w}\right) \\
< & -\left|V_{\bar{T}_{u-v}}\right| \omega(u, w) .
\end{aligned}
$$

Logo:

$$
\begin{aligned}
C\left(T_{2}\right)-C(\bar{T}) & <\left|V_{\bar{T}_{w}}\right|\left(\left|V_{\bar{T}_{u-v-w}}\right| \omega(u, w)-\left|V_{\bar{T}_{u-v}}\right| \omega(u, w)\right) \\
& =\left|V_{\bar{T}_{w}} \omega(u, w)\right|\left(\left|V_{\overline{\boldsymbol{T}}_{\boldsymbol{u}-\boldsymbol{v}-\boldsymbol{w}}}\right|-\left|\boldsymbol{V}_{\overline{\boldsymbol{T}}_{\boldsymbol{u}-\boldsymbol{v}}}\right|\right) \\
& \leq 0 .
\end{aligned}
$$

Com isto concluímos que a árvore $T^{\prime}$ obtida no final da remoção de uma aresta ruim tem custo associado menor ou igual que o da árvore $\bar{T}$ o que implica que a árvore $T$ que se obtém após remover todas as arestas ruins e é solução do MRCT com instância $I$ satisfaz $C(T) \leq C(\bar{T})$.

Observe que o custo associado a uma solução ótima $\overline{T^{*}}$ de $\bar{I}$ é sempre menor ou igual que o de uma solução ótima $T^{*}$ de $I$. Logo, se $\bar{T}$ é uma $\alpha$-aproximação para o MRCT com instância $\bar{I}$ e $T$ é a árvore que se obtém após remover as arestas ruins de $\bar{T}$, então $C(T) \leq C(\bar{T}) \leq \alpha C\left(\overline{T^{*}}\right) \leq \alpha C\left(T^{*}\right)$. Isto implica que $T$ é uma $\alpha$-aproximação para o MRCT com instância $I$. Portanto, o MRCT métrico é NP-difícil e, se existe um FPTAS para ele, então existe um FPTAS para o MRCT em grafos gerais. Como vimos na seção anterior, a menos que $\mathrm{P}=\mathrm{NP}$, o MRCT não admite FPTAS, logo o caso métrico também não.

\section{$1.4 p$-OCT}

Vamos considerar agora o $p$-OCT, um caso particular do OCT, onde para um inteiro positivo $p$ fixo é definido um conjunto $S$ de $p$ vértices fonte. Os vértices fonte são os únicos que podem ter 
requerimento positivo para outros vértices do grafo:

Problema 1.8. p-OCT - p-Source Optimum Communication spanning Tree problem.

Entrada: Um grafo $G$ com uma função não negativa de comprimento sobre as arestas, $\omega: E \rightarrow$ $\mathbb{Q}_{+}$, um sub-conjunto $S$ de $V$ com p vértices e uma função não negativa de requerimento entre os vértices de $S$ e os de $V, \psi: S \times V \rightarrow \mathbb{Q}_{+}$.

Saída: Uma árvore $T$ geradora de $G$ que minimize o custo total de comunicação:

$$
C(T)=\sum_{u \in S} \sum_{v \in V} \psi(u, v) d(T, u, v) .
$$

Se para todo par de vértices $u \in S$ e $v \in V$ temos que $\psi(u, v)=1$, então o problemas se denota $p$-MRCT. Em 2000 Farley et al. ([FFK $\left.\left.{ }^{+} 00\right]\right)$ apresentaram uma redução do 3-SAT para o 2-MRCT provando que este problema e consequentemente $p$-MRCT são também NP-difíceis. A redução usada por eles foi inspiração para a demonstração da complexidade que damos a um problema introduzido no próximo capítulo.

Dois anos depois em [Wu02], Wu apresenta outra redução, desta vez do 3-EC para o 2-MRCT métrico e em 2004 ([Wu04]), Wu mostra outra redução do 3-SAT para o 2-MRCT com pesos. O $p$-MRCT com pesos é um caso particular do $p$-OCT e uma generalização do $p$-MRCT em que para cada vértice de $S$ é definida uma função não negativa de peso $\rho: S \rightarrow \mathbb{Q}_{+}$sendo então o requerimento entre dois vértices $u \in S$ e $v \in V \psi(u, v)=\rho(u)$.

Em [Wu02] os autores percebem que o mesmo algoritmo que permite a redução do MRCT ao caso métrico é válido para reduzir o 2-MRCT para o caso métrico deste, provando com isto que não somente o $p$-MRCT, o $p$-MRCT com pesos e o $p$-OCT são NP-difíceis quando $p \geq 2$, como também as versões métricas destes problemas são NP-difíceis.

A seguir discutimos a redução dada por Wu do 3-EC para o 2-MRCT métrico.

\subsubsection{2-MRCT métrico}

Teorema 1.9. O 2-MRCT métrico é NP-difícil e, a menos que $P=N P$, este problema não admite FPTAS. ([FFK $\left.\left.{ }^{+} 00\right]\right)$

Demonstração. Considere uma instância $I=\langle S, T\rangle$ de 3-EC. A partir dela construímos o grafo completo $G=(V, E)$ da seguinte forma (a Figura 1.7 ilustra a construção deste grafo):

- Para cada $1 \leq i \leq \frac{n}{3}$, definimos o grafo $G_{i}$, que é um grafo completo no conjunto de vértices $\left\{v_{i}^{s}\right\}_{s \in S}$. Observe que o vértice $v_{i}^{s}$ corresponde ao sub-conjunto $s$ de $T$.

- Para cada $t \in T$, definimos o vértice $u_{t}$ e denotamos por $H$ o grafo completo sobre estes vértices.

- Finalmente, definimos os grafos $G_{0}$ e $G_{\frac{n}{3}+1}$ cada um com um único vértice $w_{0}$ e $w_{\frac{n}{3}+1}$, respectivamente, que são os vértices fonte.

Se consideramos $V$ o conjunto de vértices do grafo que resulta da união dos grafos acima definidos, então, definimos $G$ como o grafo completo sobre $V$. Note que cada um desses grafos é sub-grafo de $G$. Além disto, temos que o número de vértices de $G$ é $n_{G}=\frac{n}{3} m+n+2$. 
Considere um inteiro grande ${ }^{2} L \geq n_{G}\left(\frac{n}{3}+1\right)+2 \frac{n}{3}(m-1)$. Os comprimentos das arestas de $G$ são definidos da seguinte forma:

- Para cada $1 \leq i \leq \frac{n}{3}$, todas as arestas do grafo $G_{i}$ tem comprimento 1 .

- Todas as arestas do grafo $H$ também tem comprimento 1.

- Para cada $0 \leq i, j \leq \frac{n}{3}+1$, se $i \neq j$, o comprimento da aresta entre os vértices $u \in V_{G_{i}}$ e $v \in V_{G_{j}}$ é $|i-j|$. (Observe que aqui incluímos também os vértices fonte).

- Para cada $1 \leq i \leq \frac{n}{3}, s \in S$ e $t \in T$ o comprimento da aresta entre os vértices $v_{i}^{s} \in G_{i}$ e $u_{t} \in H$ é igual a $L$ se o elemento $t$ pertence ao sub-conjunto $s$, caso contrário, o comprimento da aresta é $L+1$.

- Finalmente, para cada vértice $u \in V_{H}$ o comprimento da aresta entre $u$ e $w_{0}$ é o mesmo que o da aresta entre $u$ e $w_{\frac{n}{3}+1}$ que é igual a $L+1$.

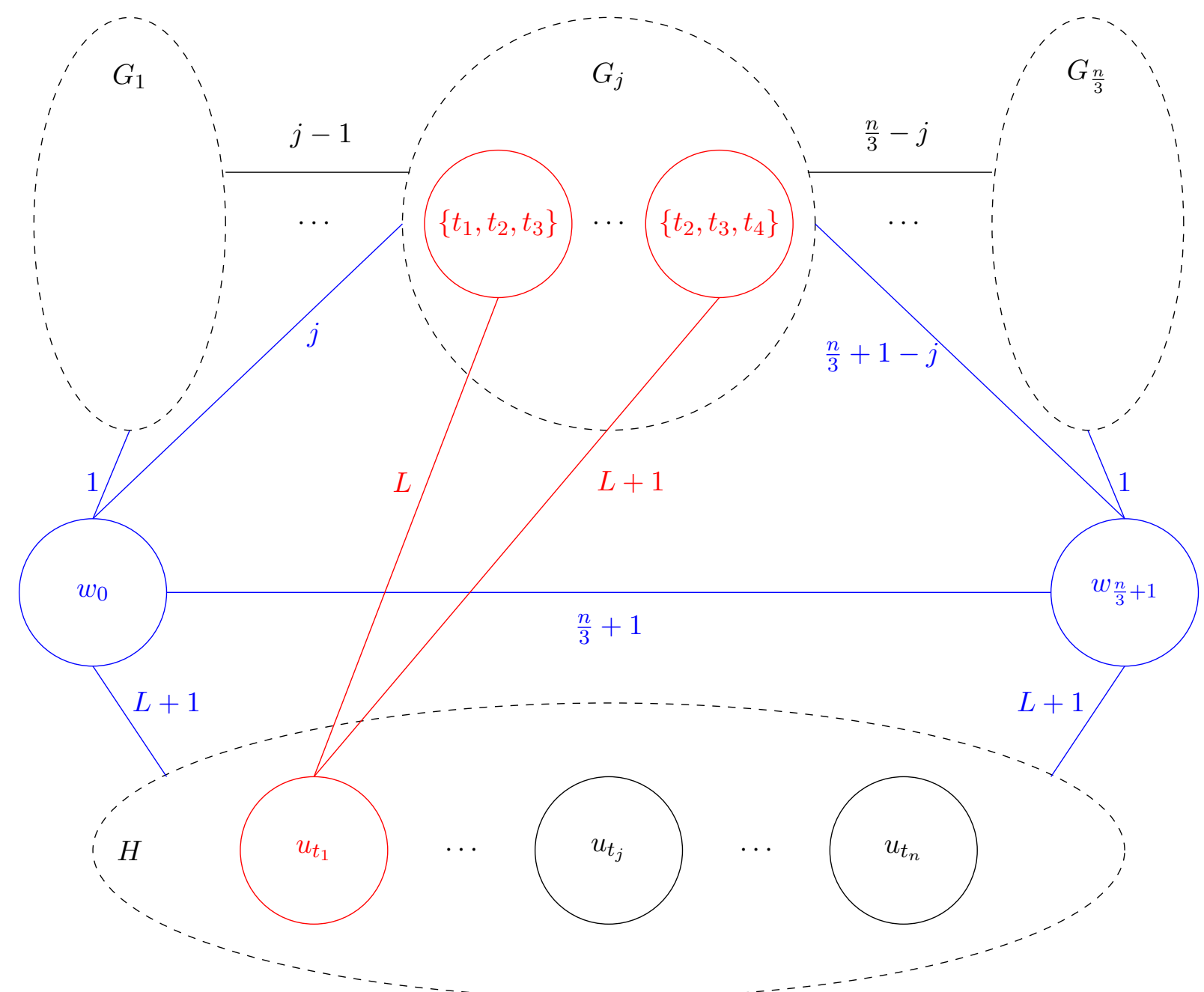

Figura 1.7: Grafo construído a partir da instância $I$ de 3-EC. Lembre que cada sub-grafo em $\left\{G_{1}, \cdots, G_{\frac{n}{3}}, H\right\}$ satisfaz que todos os vértices estão conectados por arestas de comprimento 1 . É fácil ver que esta construção garante que o grafo $G$ é métrico.

\footnotetext{
${ }^{2}$ A prova pode ser feita com valores de $L \geq \frac{n}{3}+1$, porém selecionando um valor muito maior conseguimos simplificar a análise.
} 
Não é difícil ver que o grafo $G$ construído com os comprimentos especificados para as arestas é métrico. Demonstraremos que existe uma cobertura exata para $I$ se e somente se existe uma árvore geradora $T$ de $G$ com custo de comunicação no máximo:

$$
C(T) \leq n_{G}\left(\frac{n}{3}+1\right)+2 \frac{n}{3}(m-1)+2 n L \leq(2 n+1) L .
$$

Primeiro mostraremos que se existe uma cobertura exata para $I$ então existe uma árvore geradora de $G$ que satisfaz a inequação (1.2).

Para isto, suponha que existe uma cobertura exata para $I$. Isto implica que há $\frac{n}{3}$ elementos de $S$, que são disjuntos dois a dois e cuja união é $T$. Sem perda de generalidade suponha que estes são: $s_{1}, \cdots, s \frac{n}{3}$.

Considere o caminho $P=w_{0}, v_{1}^{1}, v_{2}^{2}, \cdots, v_{\frac{n}{3}}^{\frac{n}{3}}, w_{\frac{n}{3}+1}$. Para cada vértice $u$ de $G \backslash P$, conecte $u$ a $P$ usando uma aresta de comprimento mínimo de $u$ a algum vértice de $P$. Desta forma, construímos uma árvore geradora $X$ de $G$.

Observe que todas as arestas de $P$ têm comprimento 1. Logo, o custo de comunicação a qualquer vértice de $P$ é igual ao comprimento de $P$ (pois somente os vértices nos extremos de $P$ são fontes). Isto resulta em um custo com valor $\frac{n}{3}+1$, para cada um dos $\frac{n}{3}+2$ vértices de $P$.

Note que os vértices em cada $G_{i}\left(1 \leq i \leq \frac{n}{3}\right)$ que não estão em $P$, se conectam a $P$ através de uma aresta de comprimento 1. Logo, o custo de comunicação para estes vértices é de $\frac{n}{3}+3$ (isto é, o comprimento do caminho, mais o requerimento de cada fonte passando pela aresta). Além disto, o número total destes vértices é $(m-1) \frac{n}{3}$.

Finalmente, cada vértice de $H$ se conecta a $P$ por uma aresta de comprimento $L$. Logo, o custo de comunicação para ele é de $\frac{n}{3}+1+2 L$ (o comprimento do caminho, mais o requerimento de cada fonte passando pela aresta), e temos um total de $n$ vértices em $H$.

Com estes valores o custo de $X$ é dado por:

$$
\begin{aligned}
C(X) & =\left(\frac{n}{3}+1\right)\left(\frac{n}{3}+2\right)+\left(\frac{n}{3}+3\right)(m-1) \frac{n}{3}+\left(\frac{n}{3}+1+2 L\right) n \\
& =\left(\frac{n}{3}+1\right)\left(\frac{n}{3}+2\right)+\left(\frac{n}{3}+\mathbf{1}+\mathbf{2}\right)(m-1) \frac{n}{3}+\left(\frac{n}{3}+1\right) n+\mathbf{2 n} \boldsymbol{L} \\
& =\left(\frac{n}{3}+1\right)\left(\frac{n}{3}+2\right)+\left(\frac{n}{3}+1\right)(m-1) \frac{n}{3}+\left(\frac{n}{3}+1\right) n+\mathbf{2} \frac{\boldsymbol{n}}{\mathbf{3}}(\boldsymbol{m}-\mathbf{1})+2 n L \\
& =\left(\frac{n}{3}+2+(m-1) \frac{n}{3}+n\right)\left(\frac{\boldsymbol{n}}{\mathbf{3}}+\mathbf{1}\right)+2 \frac{n}{3}(m-1)+2 n L \\
& =\boldsymbol{n}_{\boldsymbol{G}}\left(\frac{n}{3}+1\right)+2 \frac{n}{3}(m-1)+2 n L \leq(2 n+1) L .
\end{aligned}
$$

Agora analisamos a outra direção. Para isto, suponha que existe uma árvore geradora $X$ de $G$ que satisfaz a inequação (1.2).

Consideremos o caminho $P$ que une os vértices $w_{0}$ e $w_{\frac{n}{3}+1}$ em $X$. Primeiro note que nenhum vértice de $H$ pode pertencer a $P$. Para isto, perceba que o caminho mínimo dos $n$ vértices de $H$ para $w_{0}$ e para $w_{\frac{n}{3}+1}$ é $L+1$, isto por si só dá um custo de comunicação de no mínimo $2 n(L+1)$. Suponhamos que pelo menos um vértice de $H$ está em $P$, neste caso o comprimento de $P$ resulta maior ou igual do que $2(L+1)$, logo somente considerando o custo de comunicação para os vértices de $H$ e entre os próprios fontes temos um custo de: 


$$
2 n(L+1)+4(L+1)=(2 n+4)(L+1)>(2 n+1) L \geq C(X) .
$$

Portanto, não é possível ter vértices de $H$ em $P$ se $X$ satisfaz $C(X) \leq(2 n+1) L$.

Note que o custo de comunicação para cada vértice em $P$ resulta em $\omega(P)$. Logo para todos os vértices de $P$ este custo é $n_{P} \omega(P)$. Como nenhum vértice de $H$ pertence a $P$, o custo de comunicação para cada um destes vértices tem valor igual ao comprimento de $P$ mais duas vezes a distância em $X$ do vértice até $P$, isto é, $2 d(X, u, P)+\omega(P)$ para cada $u \in H$. Observe que os vértices internos de $P$ pertencem aos grafos em $\left\{G_{i}\right\}_{i=1}^{\frac{n}{3}}$, logo para cada um dos $\frac{n}{3}(m)-n_{P}$ vértices de $\bigcup_{i=1}^{\frac{n}{3}} G_{i}$ que não estão em $P$ o custo de comunicação é calculado de forma similar aos vértices de $H$, o comprimento de $P$ mais duas vezes a distância em $T$ do vértice até $P$. Com isto, o custo de $X$ resulta em:

$$
\begin{aligned}
C(X) & =n_{P} \omega(P)+\sum_{u \in H}(2 d(X, u, P)+\omega(P))+\sum_{u \in \bigcup_{i=1}^{\frac{n}{3}} G_{i} \backslash P}(2 d(X, u, P)+\omega(P)) \\
& =\left(n_{P}+n+m \frac{n}{3}-\left(n_{P}-2\right)\right) \omega(P)+2 \sum_{u \in H} d(X, u, P)+2 \sum_{u \in \bigcup_{i=1}^{\frac{n}{3}} G_{i} \backslash P} d(X, u, P) \\
& =n_{G} \omega(P)+2 \sum_{u \in H} d(X, u, P)+2 \sum_{u \in \bigcup_{i=1}^{\frac{n}{3}} G_{i} \backslash P} d(X, u, P) .
\end{aligned}
$$

Observe que $\omega(P) \geq \frac{n}{3}+1$ que é a distância mínima entre os vértices fontes em $G$. Logo, considere $\Delta_{1}=\omega(P)-\frac{n}{3}-1 \geq 0$.

$$
\begin{aligned}
C(X)= & n_{G}\left(\frac{n}{3}+1\right)+2 \sum_{u \in H} d(X, u, P)+2 \sum_{u \in \bigcup_{i=1}^{\frac{n}{3}} G_{i} \backslash P} d(X, u, P) \\
& +\Delta_{1} n_{G} .
\end{aligned}
$$

A distância de cada vértice de $H$ até $P$ é no mínimo $L$. Então, considerando para cada $u \in H$ o valor $\Delta_{2}(u)=d(X, u, P)-L \geq 0$, temos:

$$
\begin{aligned}
C(X)= & n_{G}\left(\frac{n}{3}+1\right)+2 \sum_{u \in H} L+2 \sum_{u \in \bigcup_{i=1}^{\frac{n}{3}} G_{i} \backslash P} d(X, u, P) \\
& +\Delta_{1} n_{G}+2 \sum_{u \in H} \Delta_{2}(u) \\
= & n_{G}\left(\frac{n}{3}+1\right)+2 n L+2 \sum_{u \in \bigcup_{i=1}^{\frac{n}{3}} G_{i} \backslash P} d(X, u, P) \\
& +\Delta_{1} n_{G}+2 \sum_{u \in H} \Delta_{2}(u) .
\end{aligned}
$$

Note que para cada vértice $u \in \bigcup_{i=1}^{\frac{n}{3}} G_{i} \backslash P$ a distância mínima até $P$ é 1 . Então, considerando para cada $u \in \bigcup_{i=1}^{\frac{n}{3}} G_{i} \backslash P$ o valor $\Delta_{3}(u)=d(X, u, P)-1 \geq 0$, temos: 


$$
\begin{aligned}
C(X)= & n_{G}\left(\frac{n}{3}+1\right)+2 n L+2 \sum_{u \in \bigcup_{i=1}^{\frac{n}{3}} G_{i} \backslash P} 1 \\
& +\Delta_{1} n_{G}+2 \sum_{u \in H} \Delta_{2}(u)+2 \sum_{u \in \bigcup_{i=1}^{\frac{n}{3}} G_{i} \backslash P} \Delta_{3}(u) .
\end{aligned}
$$

Além disto, como cada aresta de $G$ tem no mínimo comprimento $1,\left|\bigcup_{i=1}^{\frac{n}{3}} G_{i} \cap P\right| \leq \omega(P)=$ $\frac{n}{3}+1+\Delta_{1}$. Logo, $\left|\bigcup_{i=1}^{\frac{n}{3}} G_{i} \backslash P\right| \geq \frac{n}{3} m-\omega(P)=\frac{n}{3}(m-1)-\Delta_{1}$. Então, se consideramos $\Delta_{4}=$ $\left|\bigcup_{i=1}^{\frac{n}{3}} G_{i} \backslash P\right|-\frac{n}{3}(m-1)+\Delta_{1} \geq 0$, temos:

$$
\begin{aligned}
& C(X)=n_{G}\left(\frac{n}{3}+1\right)+2 n L+2 \frac{n}{3}(m-1) \\
& +\Delta_{1} n_{G}+2 \sum_{u \in H} \Delta_{2}(u)+2 \sum_{u \in \bigcup_{i=1}^{n} G_{i} \backslash P} \Delta_{3}(u)+2 \Delta_{4}-2 \Delta_{1} \\
& =C(X)
\end{aligned}
$$

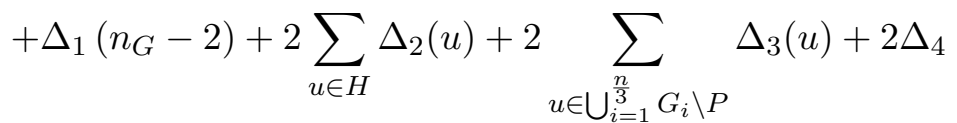

Logo, a única possibilidade é que $\Delta_{1}$ e $\Delta_{4}$ sejam iguais a zero, assim como $\Delta_{2}(u)$ para cada $u \in H$ e $\Delta_{3}(v)$ para cada $v \in \in \bigcup_{i=1}^{\frac{n}{3}} G_{i} \backslash P$.

Como $\Delta_{2}(u)$ é zero para cada $u \in H$, temos que cada elemento de $H$ está conectado a $P$ por um caminho de comprimento $L$, o que somente acontece se cada elemento de $H$ é adjacente a um elemento de $P$ por uma aresta de comprimento $L$. Isto implica que para cada $t \in T$ existe um sub-conjunto $s \in S$ que contém $t$ e há pelo menos um vértice $v_{s}^{i}$ associado a esse subconjunto no caminho $P$ (para algum $i \in\left\{1, \cdots, \frac{n}{3}\right\}$ ). Logo, os subconjuntos associados aos vértices de $P$ garantem uma cobertura de $T$.

Como $\Delta_{1}$ é zero, temos que o comprimento de $P$ é mínimo, isto é igual a $\frac{n}{3}+1$, e como toda aresta de $G$ tem comprimento pelo menos 1 , temos que em $P$ há no máximo $\frac{n}{3}$ vértices associados a subconjuntos $s \in S$. Logo, como cada subconjunto $s \in S$ tem exatamente três elementos de $T$, e há somente $\frac{n}{3}$ destes subconjuntos associados a vértices de $P$, se eles são uma cobertura de $T$, então são uma cobertura exata de $T$.

Note que nesta redução, se consideramos $L=n_{G}\left(\frac{n}{3}+1\right)+2 \frac{n}{3}(m-1)$, as instâncias obtidas para o 2-MRCT métrico garantem que os comprimentos das arestas são no máximo $O\left(n\left(m+n_{G}\right)\right)$, os requerimentos entre todo par de vértices resulta em 0,1 ou 2, e o custo de comunicação para qualquer árvore geradora é $O\left(L n_{G}^{4}\right)$. Logo, todos os parâmetros numéricos estão limitados por um polinômio no número de vértices do grafo, portanto, a menos que $\mathrm{P}=\mathrm{NP}$, o 2-MRCT métrico não admite um FPTAS. 


\subsection{PROCT e SROCT}

Após o resultado obtido em $\left[\mathrm{WLB}^{+} 98\right]$, Wu et al. continuaram trabalhando em problemas similares e introduziram outros dois casos particulares do OCT que a sua vez generalizam o MRCT implicando que ambos são NP-difíceis ([WCT00a]).

Estes problemas são o PROCT e o SROCT. Em ambos é definida uma função não negativa de peso para cada vértice do grafo $\rho: V \rightarrow \mathbb{Q}_{+}$, sendo então o requerimento entre dois vértices $u$ e $v$ de $G$ definido como o produtos dos pesos $\psi(u, v)=\rho(u) \rho(v)$ no caso do PROCT, e como a soma dos pesos $\psi(u, v)=\rho(u)+\rho(v)$ no caso do SROCT. Formalmente:

Problema 1.10. PROCT - Product Requeriment Optimum Communication spanning Tree problem.

Entrada: Um grafo $G$ com uma função não negativa de comprimento sobre as arestas, $\omega: E \rightarrow$ $\mathbb{Q}_{+}$, e uma função não negativa de requerimento sobre os vértices de $G, \rho: V \rightarrow \mathbb{Q}_{+}$. Definindo o requerimento entre dois vértices $u, v \in V$ como $\psi(u, v)=\rho(u) \rho(v)$.

Saída: Uma árvore $T$ geradora de $G$ que minimize o custo total de comunicação:

$$
C(T)=\sum_{u \in V} \sum_{v \in V} \psi(u, v) d(T, u, v)=\sum_{u \in V} \sum_{v \in V} \rho(u) \rho(v) d(T, u, v) .
$$

Problema 1.11. SROCT - Sum Requeriment Optimum Communication spanning Tree problem.

Entrada: Um grafo $G$ com uma função não negativa de comprimento sobre as arestas, $\omega: E \rightarrow$ $\mathbb{Q}_{+}$, e uma função não negativa de requerimento sobre os vértices de $G, \rho: V \rightarrow \mathbb{Q}_{+}$. Definindo o requerimento entre dois vértices $u, v \in V$ como $\psi(u, v)=\rho(u)+\rho(v)$.

Saída: Uma árvore $T$ geradora de $G$ que minimize o custo total de comunicação:

$$
C(T)=\sum_{u \in V} \sum_{v \in V} \psi(u, v) d(T, u, v)=\sum_{u \in V} \sum_{v \in V}(\rho(u)+\rho(v)) d(T, u, v) .
$$

Observe que, se no caso do PROCT o peso for unitário para todo vértice $(\rho(u)=1$ para todo $u \in V)$ teríamos o MRCT. Isso implica que o MRCT é um caso particular do PROCT. Já no SROCT, se o peso para cada vértice for um meio $\left(\rho(u)=\frac{1}{2}\right.$ para todo $\left.u \in V\right)$ teríamos novamente o MRCT o que implica que o MRCT é também um caso particular do SROCT. Além disso, se para o SROCT é dado um sub-conjunto $S$ de $p$ vértices de $G$ e para todo vértice $u$ fora de $S \rho(u)=0$, então temos uma instância do $p$-MRCT com pesos, o que implica que o SROCT é também uma generalização desse problema.

Como o MRCT e o caso métrico dele são NP-difíceis, as generalizações destes problemas também o são, portanto o PROCT, o SROCT, e os casos métricos destes problemas são NP-difíceis.

\subsection{Resumo}

A Figura 1.8 mostra a relação entre o OCT e as variantes NP-difíceis dele vistas neste capítulo. 


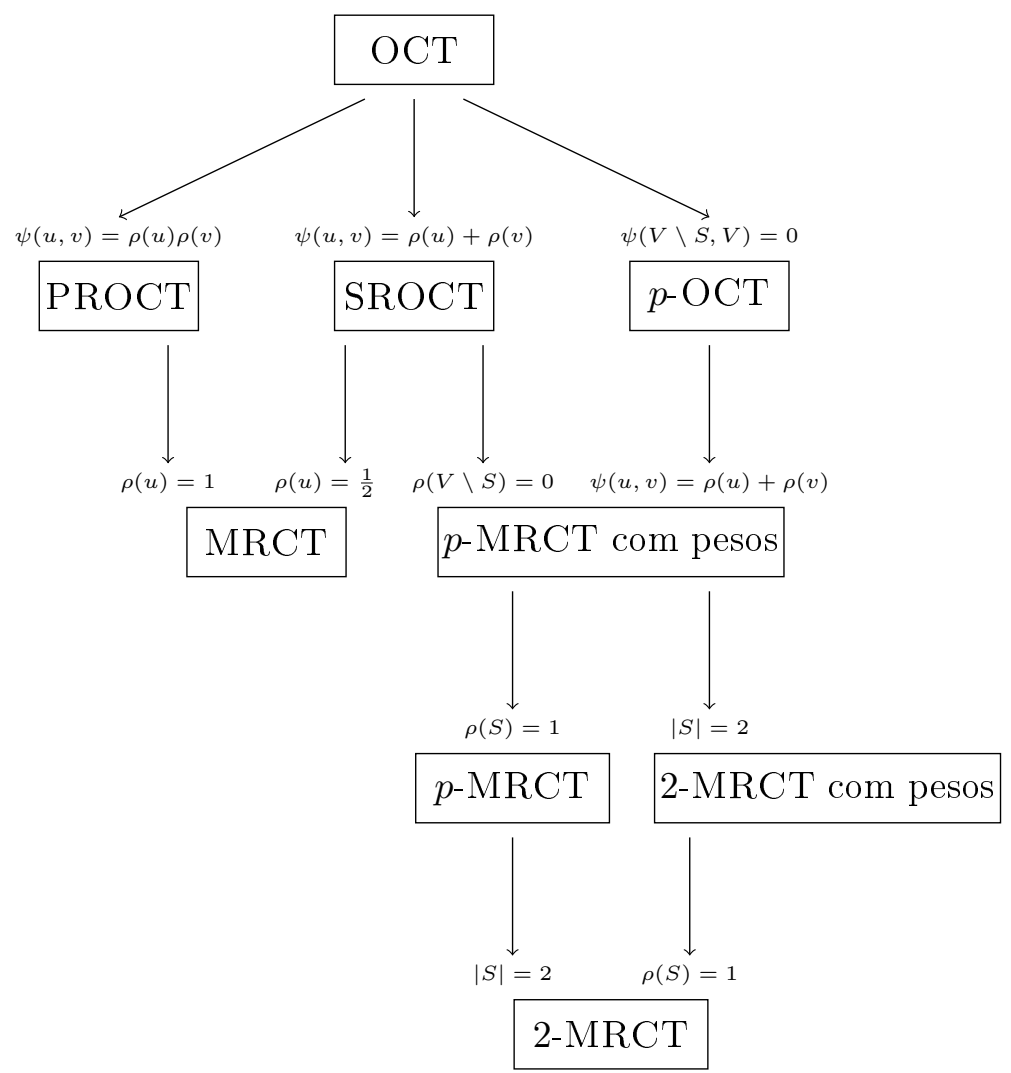

Figura 1.8: Relação entre o $\mathrm{OCT}$ e suas variantes NP-dificeis. 


\section{Capítulo 2}

\section{Novas variantes do OCT}

Seguindo com a apresentação dos problemas que são do nosso interesse e estudamos neste trabalho, neste capítulo introduzimos novas variantes NP-difíceis do OCT, onde algumas delas generalizam alguns dos casos particulares vistos no capítulo anterior.

Desta forma, na Seção 2.1 introduzimos um caso particular do $p$-OCT, o $p$-USCOCT, e provamos que não só ele, como também sua versão métrica são NP-difíceis. Na Seção 2.2 apresentamos outros casos particulares do $p$-OCT que generalizam o $p$-MRCT visto no capítulo anterior o que implica que estes problemas são também NP-difíceis. Na Seção 2.3 introduzimos o WSDOCT, um caso particular do OCT que generaliza quase todos os problemas discutidos no capítulo anterior. Finalmente, na Seção 2.4 ilustramos como fica a relação do OCT e seus casos particulares NP-difíceis com os novos problemas introduzidos.

\section{$2.1 p$-USCOCT}

O primeiro problema que introduzimos é o $p$-USCOCT, um caso particular do $p$-OCT em que o valor mínimo de requerimento entre dois vértices fonte deve ser pelo menos uma fração $(r>0)$ fixa da soma total dos requerimentos entre todos os pares de vértices:

Problema 2.1. p-USCOCT - p-Uniform Source Connecting Optimum Communication spanning Tree problem ${ }^{1}$.

Entrada: Um grafo $G$ com uma função não negativa de comprimento nas arestas, $\omega: E \rightarrow \mathbb{Q}_{+}$, um conjunto de $p$ vértices fonte $S \subseteq V$ e uma função não negativa de requerimento entre os vértices de $S$ e os de $V, \psi: S \times V \rightarrow \mathbb{Q}_{+}$, onde para cada par de vértices fonte $u, v \in S$ temos que $\psi(u, v) \geq r \sum_{u \in S} \sum_{v \in V} \psi(u, v)$.

Saída: Uma árvore $T$ geradora de $G$ que minimiza o custo total de comunicação:

$$
C(T)=\sum_{u \in S} \sum_{v \in V} \psi(u, v) d(T, u, v) .
$$

Para analisar a complexidade desta variante provaremos que o caso métrico dela (o $p$-USCOCT métrico) é NP-difícil quando $p \geq 2$, implicando que o $p$-USCOCT também é NP-difícil. Para isto, consideramos o caso em que $p=2$.

\footnotetext{
${ }^{1}$ Observe que cada constante fixa $r>0$ define uma família deste problema.
} 


\section{Prova de NP-dificuldade do 2-USCOCT métrico}

A seguir damos a definição do problema de decisão associado ao 2-USCOCT métrico (D-2USCOCT):

Problema 2.2. D-2-USCOCT

Entrada: Um valor $K \in \mathbb{Q}_{+}$, um grafo $G$, uma função de comprimento sobre as arestas do grafo, $\omega: E \rightarrow \mathbb{Q}_{+}$que satisfaz a desigualdade triangular, dois vértices fonte $u_{1}, u_{2} \in V$, uma constante fixa $r>0$ e uma função não negativa de requerimento entre os vértices fonte $e V$, $\psi:\left\{u_{1}, u_{2}\right\} \times V \rightarrow \mathbb{Q}_{+}$, onde $\min \left\{\psi\left(u_{1}, u_{2}\right), \psi\left(u_{2}, u_{1}\right)\right\} \geq r \sum_{u \in S} \sum_{v \in V} \psi(u, v)$.

Saída: Responder se existe uma árvore $T$ geradora de $G$ tal que:

$$
C(T)=\sum_{v \in V}\left(\psi\left(u_{1}, v\right) d\left(T, u_{1}, v\right)+\psi\left(u_{2}, v\right) d\left(T, u_{2}, v\right)\right) \leq K
$$

Em seguida provaremos o seguinte resultado:

Teorema 2.3. D-2-USCOCT é fortemente NP-completo.

Demonstração. Nesta prova mostramos uma redução do SAT ao D-2-USCOCT. Esta redução está inspirada na usada, a partir do 3-SAT, em [FFK $\left.{ }^{+} 00\right]$ para demonstrar que o 2-MRCT é NP-difícil.

Primeiro definimos formalmente o SAT:

Problema 2.4. SAT - Boolean Satisfiability problem.

Entrada: Um conjunto de cláusulas $\left\{C_{j}\right\}_{j=1}^{m}$ envolvendo literais sobre o conjunto de variáveis booleanas $\left\{x_{i}\right\}_{i=1}^{n}$.

Saída: Responder se existe uma atribuição de valores às variáveis que satisfaça todas as cláusulas.

Dada uma instância $I=\left\langle\left\{x_{i}\right\}_{i=1}^{n},\left\{C_{j}\right\}_{j=1}^{m}\right\rangle$ do SAT, definimos o grafo $G$ como segue:

- $V_{G}$ :

- Para cada variável $x_{i}(i \in\{1, \cdots, n\})$ tome os vértices $x_{i}^{T}$ e $x_{i}^{F}$,

- Para cada $i \in\{1, \cdots, n-1\}$ defina o vértice $v_{i, i+1}$,

- Para cada cláusula $C_{j}(j \in\{1, \cdots, m\})$ tome o vértice $c_{j}$,

- Considere também os dois vértices fonte $u_{1}$ e $u_{2}$.

- $E_{G}$ :

- Para cada $i(i \in\{1, \cdots, n-1\})$ inclua as arestas $x_{i}^{T} v_{i, i+1}$ e $x_{i}^{F} v_{i, i+1}$,

- Para cada $i(i \in\{2, \cdots, n\})$ adicione as $\operatorname{arestas} x_{i}^{T} v_{i-1, i}$ e $x_{i}^{F} v_{i-1, i}$,

- Para cada $j$ e $i(j \in\{1, \cdots, m\}, i \in\{1, \cdots, n\})$, se a cláusula $C_{j}$ contém o literal $x_{i}$ então adicione a aresta $c_{j} x_{i}^{T}$, e se a cláusula $C_{j}$ contém o literal $\neg x_{i}$ então adicione a aresta $c_{j} x_{i}^{F}$

- Finalmente adicione arestas $u_{1} x_{1}^{T}, u_{1} x_{1}^{F}, u_{2} x_{n}^{T}$ e $u_{2} x_{n}^{F}$, que conectam os vértices fonte ao restante do grafo. 

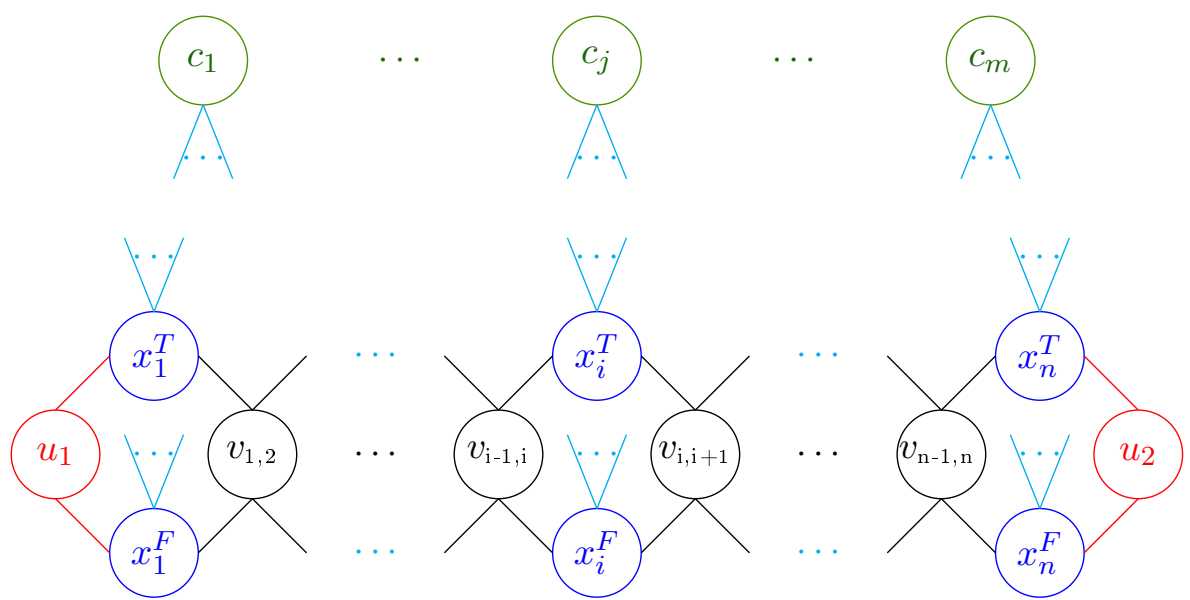

Figura 2.1: Grafo $G$ obtido de I. Observe que para cada $j \in\{1, \cdots, m\}$ os vértices $c_{j}$ somente são adjacentes em $G$ a vértices $x_{i}^{F}$ ou $x_{i}^{T}$ para alguns valores de $i \in\{1, \cdots, n\}$.

Todas as arestas adjacentes a vértices do tipo $c_{j}$, para $j \in\{1, \cdots, m\}$, terão comprimento $2 n$ e o restante das arestas terá associado comprimento 1 . Formalmente $\omega: E_{G} \rightarrow \mathbb{Q}$ é definida:

$$
\omega(u v)= \begin{cases}1 & \text { se } u, v \notin\left\{c_{j}\right\}_{j=1}^{m} \\ 2 n & \text { caso contrário }\end{cases}
$$

Defina, então, o grafo completo $G^{\prime}$ sobre o mesmo conjunto de vértices de $G$, de forma que o comprimento das arestas $\omega^{\prime}: E_{G^{\prime}} \rightarrow \mathbb{Q}$ seja: $\omega^{\prime}(u v)=d(G, u, v)$. É fácil ver que $G^{\prime}$ é métrico pois da forma em que definimos a função $\omega^{\prime}$, o par $\left\langle G^{\prime}, \omega^{\prime}\right\rangle$ é fecho métrico do $\langle G, \omega\rangle$. Além disso, qualquer caminho mínimo entre $u_{1}$ e $u_{2}$ tem comprimento $2 n$ (isto em ambos os grafos, devido ao fato da distância entre estes vértices em $G$ ser $2 n$ ). Definimos, então, a função de requerimento: $\psi: V_{G^{\prime}} \rightarrow \mathbb{Q}:$

$$
\psi(u, v)= \begin{cases}1 & \text { se } u \in\left\{u_{1}, u_{2}\right\}, v \in\left\{c_{j}\right\}_{j=1}^{m} \\ 3 n m & \text { se }(u, v) \in\left\{\left(u_{1}, u_{2}\right),\left(u_{2}, u_{1}\right)\right\} \\ 0 & \text { caso contrário }\end{cases}
$$

Finalmente, tome $K=12 m n^{2}+6 m n$ e $r=\frac{3}{8}$. Com isso, definimos a instância $I^{\prime}=\left\langle K, G^{\prime}, \omega^{\prime}, r, \psi\right\rangle$ do D-2-USCOCT associada à instância $I$ do SAT. Observe que $I^{\prime}$ satisfaz:

$$
\min \left\{\psi\left(u_{1}, u_{2}\right), \psi\left(u_{2}, u_{1}\right)\right\}=3 m n=\frac{3}{8} 8 m n \geq \frac{3}{8}(6 m n+2 m)=r \sum_{u \in S} \sum_{v \in V} \psi(u, v) .
$$

Primeiro provamos que, se existe uma árvore geradora $T$ de $G^{\prime}$ que dá uma resposta positiva para D-2-USCOCT com instância $I^{\prime}$, então existe uma atribuição de valores às variáveis de $I$ que dá resposta positiva para SAT com instância $I$.

Para isso, suponha que existe uma árvore geradora $T$ de $G^{\prime}$ tal que $C(T) \leq K$.

Se o caminho entre $u_{1}$ e $u_{2}$ em $T$ não é um caminho de comprimento mínimo entre $u_{1}$ e $u_{2}$ em $G^{\prime}$, então $d\left(T, u_{1}, u_{2}\right)>d\left(G^{\prime}, u_{1}, u_{2}\right)=2 n$, ou seja $d\left(T, u_{1}, u_{2}\right) \geq 2 n+1$. Logo:

$$
C(T)=\sum_{v \in V}\left(\psi\left(u_{1}, v\right) d\left(T, u_{1}, v\right)+\psi\left(u_{2}, v\right) d\left(T, u_{2}, v\right)\right)
$$




$$
\begin{aligned}
& =\left(3 m n d\left(T, u_{1}, u_{2}\right)+3 m n d\left(T, u_{2}, u_{1}\right)\right)+\sum_{j=1}^{m}\left(d\left(T, u_{1}, c_{j}\right)+d\left(T, u_{2}, c_{j}\right)\right) \\
& >3 m n\left(d\left(T, u_{1}, u_{2}\right)+d\left(T, u_{2}, u_{1}\right)\right) \geq 3 m n((2 n+1)+(2 n+1))=K
\end{aligned}
$$

Portanto, o caminho entre $u_{1}$ e $u_{2}$ em $T$ é um caminho mínimo entre esses vértices em $G^{\prime}$, isto é $d\left(T, u_{1}, u_{2}\right)=d\left(G^{\prime}, u_{1}, u_{2}\right)=2 n$. logo:

$$
C(T)=12 m n^{2}+\sum_{j=1}^{m}\left(d\left(T, u_{1}, c_{j}\right)+d\left(T, u_{2}, c_{j}\right)\right) .
$$

Como, em $G^{\prime}$, para todo $j \in\{1, \cdots, m\}$ o comprimento de qualquer caminho entre $c_{j}$ e $u_{1}$ ou $c_{j}$ e $u_{2}$ é maior que $2 n$, então $c_{j}$ não pertence ao caminho entre $u_{1}$ e $u_{2}$ em $T$. Logo, se para cada $j \in\{1, \cdots, m\}$ denotamos por $y_{j}$ o vértice no caminho entre $u_{1}$ e $u_{2}$ em $T$ mais próximo do vértice $c_{j}$, então:

$$
C(T)=12 m n^{2}+\sum_{j=1}^{m}\left(d\left(T, u_{1}, y_{j}\right)+d\left(T, u_{2}, y_{j}\right)+2 d\left(T, y_{j}, c_{j}\right)\right) .
$$

Como $y_{j}$ pertence ao caminho entre $u_{1}$ e $u_{2}$ em $T$, temos que $d\left(T, u_{1}, y_{j}\right)+d\left(T, u_{2}, y_{j}\right)=$ $d\left(T, u_{1}, u_{2}\right)=2 n$. Logo:

$$
C(T)=12 m n^{2}+\sum_{j=1}^{m}\left(2 n+2 d\left(T, y_{j}, c_{j}\right)\right)=12 m n^{2}+2 m n+\sum_{j=1}^{m} 2 d\left(T, y_{j}, c_{j}\right) .
$$

Pela nossa hipótese $(C(T) \leq K)$, segue que $\sum_{j=1}^{m} 2 d\left(T, y_{j}, c_{j}\right) \leq 4 m n$. Mas, para cada $j \in$ $\{1, \cdots, m\}$ a distância entre $c_{j}$ e qualquer outro vértice $u \in V_{G}$ é pelo menos $2 n$, o que implica que $\sum_{j=1}^{m} 2 d\left(T, y_{j}, c_{j}\right) \geq 4 m n$. Logo, para que $\sum_{j=1}^{m} 2 d\left(T, y_{j}, c_{j}\right) \leq 4 m n$, é necessário que para cada $j \in\{1, \cdots, m\}$ a distância entre $c_{j}$ e $y_{j}$ seja exatamente igual a $2 n$ o que somente acontece se $y_{j}$ é adjacente a $c_{j}$ em $T$. Daí, para algum $i \in\{1, \cdots, n\}, y_{j}=x_{i}^{T}$ sendo $x_{i}$ um literal da cláusula $C_{j}$ ou $y_{j}=x_{i}^{F}$ sendo $\neg x_{i}$ um literal da cláusula $C_{j}$.

Logo, para cada $i \in\{1, \cdots, n\}$ se $x_{i}^{T}$ pertence ao caminho entre $u_{1}$ e $u_{2}$ em $T$, então a variável $x_{i}$ é verdadeira, caso contrário ela é falsa (Observe que para todo $i \in\{1, \cdots, n\}, d\left(G^{\prime}, u_{1}, x_{i}^{T}\right)+$ $d\left(G^{\prime}, u_{2}, x_{i}^{F}\right)=d\left(G^{\prime}, u_{1}, x_{i}^{F}\right)+d\left(G^{\prime}, u_{2}, x_{i}^{T}\right) \geq 2 n$ o que implica que somente um vértice entre $x_{i}^{T}$ e $x_{i}^{F}$ pode estar no caminho entre $u_{1}$ e $\left.u_{2}\right)$.

Assim, para cada $j \in\{1, \cdots, m\}$ se o elemento $y_{j}=x_{i}^{T}$ para algum $i \in\{1, \cdots, n\}$ então a cláusula $C_{j}$ contém o literal $x_{i}$ que é verdadeiro, portanto a cláusula $C_{j}$ é satisfeita; caso contrário $y_{j}=x_{i}^{F}$, para algum $i \in\{1, \cdots, n\}$ o que implica que a cláusula $C_{j}$ contém o literal $\neg x_{i}$, sendo $x_{i}$ falso, portanto neste caso a cláusula $C_{j}$ também é satisfeita. Logo, pode-se concluir que se existe uma árvore $T$ geradora de $G^{\prime} \operatorname{com} C(T) \leq K$ então existe uma atribuição de valores às variáveis que satisfaz todas as cláusulas de $I$.

Agora será demonstrada a outra direção. Para isso, suponha que existe uma atribuição de valores $A$ para as variáveis de $I$, tal que todas as cláusulas de $I$ são satisfeitas. A partir dela construímos a seguinte árvore $T$ que satisfaz também a condição de $I^{\prime}$.

- Primeiro, defina o caminho entre $u_{1}$ e $u_{2}$ em $T$ : Para isso considere um caminho $P$ que começa em $u_{1}$ e chega até $u_{2}$ passando por todos os vértices $v_{i, i+1}(i \in\{1, \cdots, n-1\})$, isto 
é: $P=u_{1} v_{1,2} v_{2,3} \ldots v_{n-1, n} u_{2}$. Considerando $u_{1}=v_{0,1}$ e $u_{2}=v_{n, n+1}$, para cada $i \in\{1, \cdots, n\}$ coloque entre os vértices $v_{i-1, i}$ e $v_{i, i+1}$, no caminho $P$, o vértice $x_{i}^{T}$ se $x_{i}$ é verdadeira em $A$, caso contrário coloque o vértice $x_{i}^{F}$. Observe que com isso o comprimento de $P$ é $2 n$, que é o comprimento de um caminho mínimo entre $u_{1}$ e $u_{2}$ em $G^{\prime}$.

- Como a atribuição de valores $A$ satisfaz $I$, então para cada $j \in\{1, \cdots, m\}$ existe um literal $Y_{j}$ de $C_{j}$ cujo valor é verdade em $A$. Seja $x_{i}$ a variável do literal $Y_{j}$ (para algum $i \in\{1, \cdots, n\}$ ), se $Y_{j}=x_{i}$ então adicione a aresta $c_{j} x_{i}^{T}$ a $T$, que tem comprimento $2 n$ e como $Y_{j}$ é verdadeiro então $x_{i}^{T}$ pertence a $P$. Caso contrário, temos que $Y_{j}=\neg x_{i}$, logo adicionamos a aresta $c_{j} x_{i}^{F}$ a $T$ que também tem comprimento $2 n$ e como $Y_{j}$ é verdadeiro, então $x_{i}^{F}$ pertence a $P$. Note que com isto se garante que: $\sum_{j=1}^{m} 2 d\left(T, y_{j}, c_{j}\right)=\sum_{j=1}^{m} 4 n \leq 4 m n$.

- Como o restante dos vértices não têm requerimentos positivos associados, eles podem ser adicionados a $T$ como folhas adjacentes a qualquer outro vértice, pois não interferem no valor de $C(T)$.

A construção acima resulta em uma árvore geradora $T$ de $G^{\prime}$ que satisfaz $C(T) \leq K$, e com isto concluímos a redução do SAT ao D-2-USCOCT.

Como visto no capítulo anterior, o problema de decisão associado a OCT pertence à classe NP, o que é válido para todos os casos particulares dele. Portanto, o D-2-USCOCT também pertence a NP e como acabamos de reduzir polinomialmente o SAT (um problema NP-completo) ao D-2USCOCT, temos que este último é também NP-completo.

Observe que as arestas de $G$ tem no máximo comprimento $2 n$, portanto o comprimento das arestas de $G^{\prime}$ resulta em $O\left(n n_{G^{\prime}}\right)$. Alem disto, o requerimento entre vértices não fonte do grafo é 0 , entre um vértice fonte e um não fonte 1 e entre dois fonte é $O(m n)$ e o valor da constante $K=$ $12 m n^{2}+6 m n$. Logo, D-2-USCOCT métrico é NP-completo inclusive quando todos os parâmetros numéricos de sua estão limitados por um polinômio no número de vértices, portanto D-2-USCOCT métrico é fortemente NP-completo.

Assim, podemos concluir que o 2-USCOCT métrico é NP-difícil e não admite FPTAS, portanto, para $p \geq 2$, o $p$-USCOCT e sua versão métrica também são NP-difíceis e não admitem FPTAS.

A seguir introduzimos outras duas variantes do $p$-OCT.

\section{$2.2 p$-WSDOCT e $p$-WDOCT}

Um outro caso particular do $p$-OCT que apresentamos é o $p$-WDOCT, onde definimos para cada vértice um requerimento de recepção, sendo o requerimento entre uma fonte e um vértice igual ao requerimento de recepção deste último. Formalmente:

Problema 2.5. p-WDOCT - p-source Weighted Destination Optimum Communication spanning Tree problem.

Entrada: Um grafo $G$ com uma função não negativa de comprimento sobre as arestas, $\omega: E \rightarrow$ $\mathbb{Q}_{+}$, um subconjunto $S$ de $V$ com p vértices e uma função não negativa de requerimento de recepção para cada vértice de $V \rho: V \rightarrow \mathbb{Q}_{+}$. O requerimento entre os vértices $u \in S$ e $v \in V$ é dado por $\psi(u, v)=\rho(v)$. 
Saída: Uma árvore geradora $T$ de $G$ que minimize o custo total de comunicação:

$$
C(T)=\sum_{u \in S} \sum_{v \in V} \psi(u, v) d(T, u, v)=\sum_{u \in S} \sum_{v \in V} \rho(v) d(T, u, v) .
$$

Não é difícil perceber que quando o requerimento de recepção é unitário para todos os vértices estamos no caso do $p$-MRCT, o que implica que o $p$-WDOCT é uma generalização dele e portanto tanto ele quanto sua versão métrica são NP-difíceis.

Outro problema que também introduzimos nesta seção é o $p$-WSDOCT, que também é um caso particular do $p$-OCT e generaliza o $p$-WDOCT. Neste problema, a cada vértice fonte lhe é atribuído um requerimento de envio, sendo então o requerimento entre um fonte e outro vértice o produto dos requerimentos de envio (da fonte) pelo de recepção (do outro vértice). Uma definição formal é:

Problema 2.6. $p$-WSDOCT - p-source Weighted Source Destination Optimum Communication spanning Tree problem.

Entrada: Um grafo $G$ com uma função não negativa de comprimento sobre as arestas, $\omega$ : $E \rightarrow \mathbb{Q}_{+}$, um sub-conjunto $S$ de $V$ com $p$ vértices, uma função não negativa de requerimento de envio para cada vértice de $S, \sigma: S \rightarrow \mathbb{Q}_{+}$e uma função não negativa de requerimento de recepção para cada vértice de $V \rho: V \rightarrow \mathbb{Q}_{+}$. O requerimento entre os vértices $u \in S$ e $v \in V$ é dado por $\psi(u, v)=\sigma(u) \rho(v)$.

Saída: Uma árvore geradora $T$ de $G$ que minimize o custo total de comunicação:

$$
C(T)=\sum_{u \in S} \sum_{v \in V} \psi(u, v) d(T, u, v)=\sum_{u \in S} \sum_{v \in V} \sigma(u) \rho(v) d(T, u, v)
$$

Como este problema generaliza o $p$-WDOCT, também generaliza o $p$-MRCT e portanto ele e sua versão métrica são também NP-difíceis. Além disso, temos que o $p$-MRCT com pesos é o caso particular em que o requerimento de recepção é unitário para todos os vértices.

A próxima seção introduz um novo problema que generaliza os desta seção, além de também generalizar vários dos que foram tratados anteriormente na literatura.

\subsection{WSDOCT}

Finalmente introduzimos o WSDOCT. Este caso particular do OCT generaliza vários dos problemas apresentados previamente: PROCT, SROCT, $p$-WSDOCT e portanto as variantes particulares de cada um deles.

Problema 2.7. WSDOCT - Weighted Source Destination Optimum Communication spanning Tree problem.

Entrada: Um grafo $G$ com uma função não negativa de comprimento sobre as arestas, $\omega: E \rightarrow$ $\mathbb{Q}_{+}$, uma função não negativa de requerimento de envio para cada vértice do grafo, $\sigma: V \rightarrow \mathbb{Q}_{+}$ e uma função não negativa de requerimento de recepção para cada vértice de $V \rho: V \rightarrow \mathbb{Q}_{+}$. $O$ requerimento entre os vértices $u, v \in V$ é dado por $\psi(u, v)=\sigma(u) \rho(v)$.

Saída: Uma árvore geradora $T$ de $G$ que minimize o custo total de comunicação:

$$
C(T)=\sum_{u \in V} \sum_{v \in V} \psi(u, v) d(T, u, v)=\sum_{u \in V} \sum_{v \in V} \sigma(u) \rho(v) d(T, u, v)
$$


Não é difícil perceber que o $p$-WSDOCT é o caso particular do WSDOCT em que, dado o subconjunto $S$ de vértices de $G$, a função de requerimento de envio é igual a zero para os vértices fora de $S$, isto é, $\sigma: V-S \rightarrow 0$.

Observe que também o SROCT e o PROCT são casos particulares deste problema. O SROCT é o caso em que a função de envio é unitária para todos os vértices de $G$, isto é $\sigma: V \rightarrow 1$; enquanto o PROCT é o caso em as funções de envio e requerimento são iguais, isto é, para cada $u \in V$ $\sigma(u)=\rho(u)$. Logo, tanto o WSDOCT quanto sua versão métrica são NP-difíceis.

Em seguida mostramos como fica a relação entre o OCT e suas variantes considerando os problemas introduzidos neste capítulo.

\subsection{Resumo}

Similar ao resumo do capítulo anterior, mostramos aqui a relação entre os problemas. Observe que dentre os problemas que têm sido de interesse pela literatura e os aqui apresentados o WSDOCT somente não generaliza o próprio OCT, o p-OCT e o $p$-USCOCT. Todos os demais problemas são casos particulares dele. Também é importante perceber que todos os problemas aqui relacionados são NP-difíceis inclusive quando restringimos para grafos métricos.

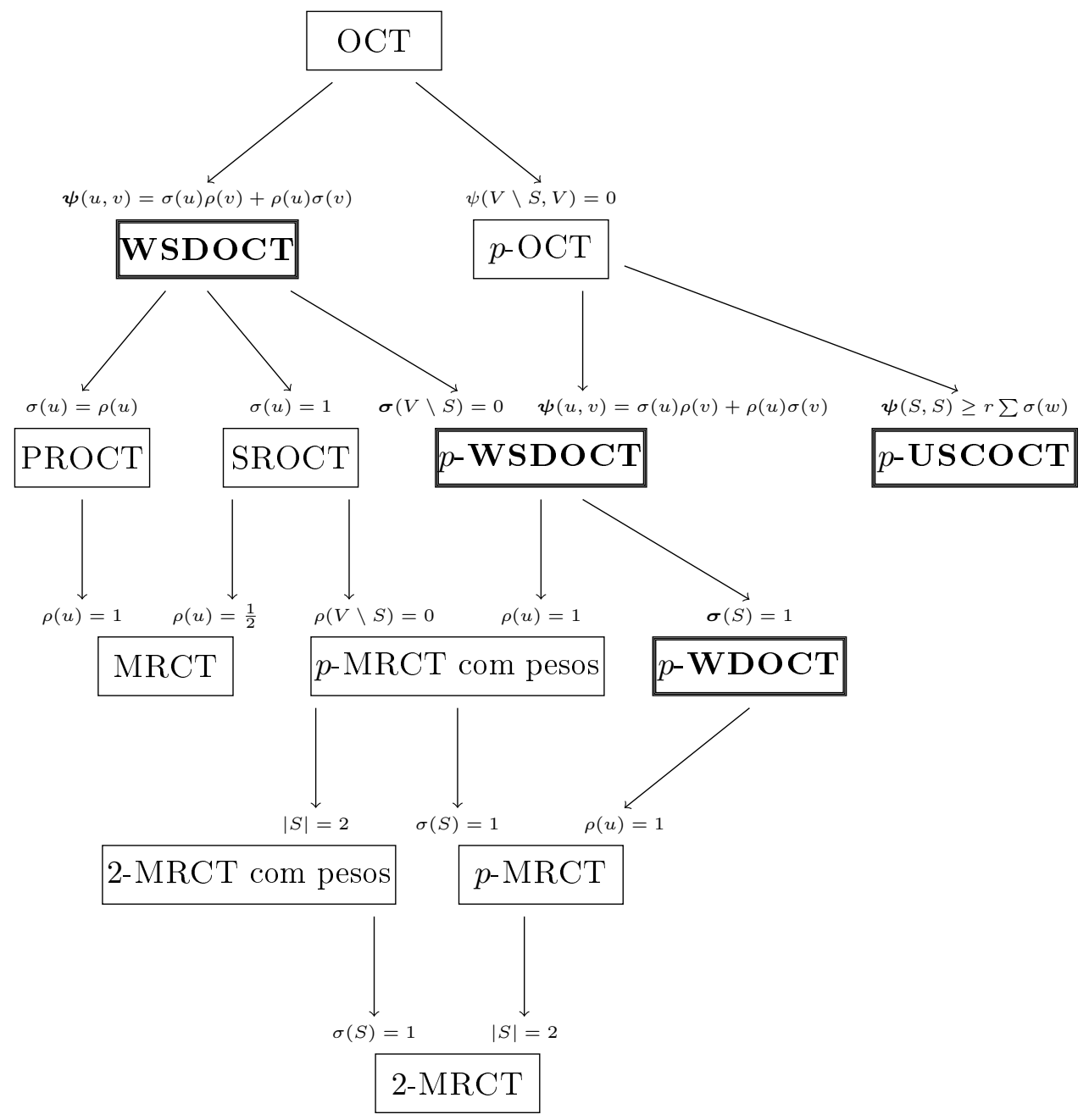

Figura 2.2: Relação entre o OCT e suas variantes NP-difíceis. Os problemas em negrito são os introduzidos neste capítulo. 


\section{Capítulo 3}

\section{Resultados de aproximação na literatura}

Neste capítulo apresentamos os principais resultados sobre aproximação que podem ser encontrados na literatura para o OCT e suas diferentes versões.

Assim, na Seção 3.1 discutimos o PTAS para o MRCT apresentado por Wu et. al em 1998 ([WLB $\left.\left.{ }^{+} 98\right]\right)$. Nesse mesmo artigo os autores dão uma $O\left(\log ^{2} n\right)$-aproximação para o OCT que discutimos na Seção 3.2. Na Seção 3.3 discutimos sobre as aproximações obtidas para o PROCT e para o SROCT ([WCT00a, WCT00b]). Na Seção 3.4 analisamos os diferentes resultados de aproximação que foram dados para o $p$-OCT e seus casos particulares ([Wu02, Wu04]). Finalmente na Seção 3.5 enriquecemos a relação entre estes problemas adicionando as melhores aproximações encontradas para eles.

\subsection{PTAS para o MRCT}

O primeiro resultado para o MRCT foi dado por Wu et al. em 1998 [WLB ${ }^{+}$98]. Os autores não só mostram uma redução do MRCT para sua versão métrica nesse trabalho, como visto na seção 1.3, como oferecem um PTAS para o MRCT métrico o que também representa um PTAS para o MRCT com grafos gerais.

A ideia deste algoritmo é reduzir o problema de encontrar a árvore geradora ótima ao de encontrar o $k$-centro ${ }^{1}$ ótimo para $k$ fixo. Um $k$-centro é um tipo de árvore geradora de $G$, em que o número de vértices internos está limitado por um inteiro positivo $k$, o que significa que a árvore tem no mínimo $n-k$ folhas. O PTAS apresentado pelos autores garante uma $(1+\epsilon)$-aproximação em tempo $O\left(n^{2\left\lceil\frac{2}{\epsilon}\right\rceil-2}\right)$. O mesmo é um caso particular do que apresentamos nos capítulos 5 e 6 .

Além de apresentar esta aproximação para o MRCT, no mesmo trabalho, os autores mostram uma $O\left(\log ^{2} n\right)$ aproximação para o OCT.

\subsection{Aproximação para $\mathrm{OCT}$}

Nesta seção discutimos sobre a aproximação apresentada em $\left[\mathrm{WLB}^{+} 98\right]$ para o OCT. Para isto, precisamos introduzir algumas noções de métricas sobre um conjunto de pontos $V$.

Definição 3.1. Dada uma métrica $M$ sobre $V$, denotamos por $d_{M}(i, j)$ a distância em $M$ entre os pontos $i$ e $j$ de $V$.

\footnotetext{
${ }^{1}$ Os autores usam o termo $k$-estrela, porém este pode facilmente ser confundido com o conceito usual de $k$-estrela em teoria dos grafos que é uma árvore com um único vértice interno e $k$ folhas.
} 
Definição 3.2. Dizemos que uma métrica $N$ sobre $V$ domina uma outra métrica $M$ sobre $V$ se para qualquer par de elementos $i, j \in V$, temos que $d_{N}(i, j) \geq d_{M}(i, j)$.

Definição 3.3. Dizemos que uma métrica $N$ sobre $V$-aproxima uma métrica $M$ sobre $V$ se $N$ domina $M$ e para qualquer par de elementos $i, j \in V$, temos que $d_{N}(i, j) \leq \alpha d_{M}(i, j)$.

Definição 3.4. Um conjunto $S$ de métricas sobre $G \alpha$-aproxima-probabilisticamente uma métrica $M$ sobre $V$, se toda métrica de $S$ domina $M$ e existe uma distribuição de probabilidade sobre as métricas $N \in S$ tal que para todo par de pontos $i$ e $j$ de $V$ a esperança $E\left(d_{N}(i, j)\right)$ satisfaz: $E\left(d_{N}(i, j)\right) \leq \alpha d_{M}(i, j)$.

Observe que toda árvore, com função de comprimento nas arestas, define uma métrica sobre os vértices dela, onde a distância entre dois vértices na métrica é justamente a distância entre eles na árvore.

Se consideramos uma instância $I=\langle G, \omega, \psi\rangle$ do OCT temos que uma solução ótima é uma árvore geradora $T^{*}$ de $G$, e pelo que acabamos de observar ela é também uma métrica sobre os vértices de $G$, sendo o nosso objetivo aproximar a mesma. O seguinte teorema de Bartal [Bar96] nos ajuda nisso.

Teorema 3.5. Qualquer métrica sobre $V$ pode ser $O\left(\log ^{2}|V|\right)$-aproximada probabilisticamente por um conjunto de métricas definidas por árvores sobre $V$. Ainda, esse conjunto de métricas e a distribuição de probabilidades sobre elas podem ser computados em tempo polinomial.

Em [KRS97] foi observado que o conjunto de árvores que definem as métricas neste teorema pode ser um conjunto de árvores geradoras. Logo, podemos encontrar em tempo polinomial um conjunto $S$ de árvores geradoras de $G$, cujas métricas $O\left(\log ^{2}|V|\right)$-aproximem probabilisticamente $T^{*}$. Isto implica que, para qualquer árvore geradora $T \in G$ e qualquer par de vértices $u$ e $v$ de $G$ $E(d(T, u, v)) \leq O\left(\log ^{2}|V|\right) d\left(T^{*}, u, v\right)=O\left(\log ^{2} n\right) d\left(T^{*}, u, v\right)$, logo:

$$
\begin{aligned}
E(C(T)) & =E\left(\sum_{u \in V_{G}} \sum_{v \in V_{G}} \psi(u, v) d(T, u, v)\right) \\
& =\sum_{u \in V_{G}} \sum_{v \in V_{G}} \psi(u, v) E(d(T, u, v)) \\
& \leq \sum_{u \in V_{G}} \sum_{v \in V_{G}} \psi(u, v) \boldsymbol{O}\left(\log ^{2} \boldsymbol{n}\right) \boldsymbol{d}\left(\boldsymbol{T}^{*}, \boldsymbol{u}, \boldsymbol{v}\right) \\
& =O\left(\log ^{2} n\right) \sum_{u \in V_{G}} \sum_{v \in V_{G}} \psi(u, v) d\left(T^{*}, u, v\right) \\
& =O\left(\log ^{2} n\right) C\left(T^{*}\right) .
\end{aligned}
$$

Com isto temos uma $O\left(\log ^{2} n\right)$-aproximação probabilística para o problema. Ainda, em [Bar98, CCGG98] foi dada uma razão de aproximação melhor com valor $O(\log n \log \log n)$, mostrando também como desaleatorizar resultados deste tipo. Finalmente, em 2003, Talwar et al. ([TFR03]) melhoram o resultado de Bartal para uma $O(\log n)$-aproximação. Esta é a melhor aproximação conhecida para o OCT. 


\subsection{Aproximações para o SROCT e o PROCT}

Nesta seção apresentamos os resultado para SROCT e PROCT dados por Wu et al. em [WCT00a]. Neste artigo, os autores apresentam os dois problemas obtendo uma 2-aproximação com tempo $O\left(n^{3}\right)$ para o SROCT e uma 1.577-aproximação com tempo $O\left(n^{5}\right)$ para o PROCT.

No caso do PROCT os autores percebem que o algoritmo por trás da redução do MRCT para MRCT métrico, apresentada na seção 1.3, se aplica também para reduzir o PROCT para seu caso métrico mantendo a mesma razão de aproximação, com praticamente a mesma demonstração. Porém, no caso do SROCT esta propriedade não se aplica.

Ainda, no mesmo ano em [WCT00b], os autores melhoraram a razão de aproximação para o PROCT encontrando um PTAS que dá uma $(1+\epsilon)$-aproximação para o PROCT métrico em tempo $O\left(k^{k-1} n^{2 k}\left(\frac{q}{\lambda}\right)^{k}\right)$, onde $\epsilon=\frac{2}{k+1}+\left(\frac{2}{q}+\frac{1}{q^{2}}+\lambda \frac{(k+3)^{3}}{k+1}\right) \frac{k+3}{k+1}$, com $k$ e $q$ inteiros positivos e $\lambda$ um valor positivo menor que 1. Esta solução é um caso particular do PTAS que propomos nos capítulos 5 e 6.

A seguir, discutimos a 2-aproximação para o SROCT. A prova para a 1.577-aproximação do PROCT baseia-se em ideias semelhantes.

A ideia para esta 2-aproximação é dada uma instância $I=\langle G, \omega, \rho\rangle$ do SROCT, encontrar para cada vértice $u$ de $G$ uma árvore geradora $T_{u}$ de $G$, tal que a distância em $T_{u}$ de $u$ para qualquer outro vértice de $G$ seja mínima, isto é, para cada $v \in V$ se satisfaz $d(T, u, v)=d(G, u, v)$. Selecionamos, então, dentre as $n$ árvores encontradas a de menor custo.

Encontrar $T_{u}$ associada a um vértices $u \in V$ pode ser feito em tempo $O\left(n^{2}\right)$. Logo, podemos encontrar estas árvores para cada vértice do grafo em tempo $O\left(n^{3}\right)$. Também podemos calcular o custo de todas elas em igual tempo, assim como selecionar a de menor custo. Portanto, o algoritmo proposto consome tempo $O\left(n^{3}\right)$.

Agora veremos que a solução dada por este algoritmo é de fato uma 2-aproximação para SROCT.

Para isto, considere uma árvore geradora $T$ de $G$ e um vértice qualquer $w$ de $T$.

Com o objetivo de facilitar a leitura denotemos por $\mathbb{P}$ a soma dos requerimentos de todos os vértices do grafo $\left(\mathbb{P}=\sum_{u \in V} \rho(u)\right)$.

Provemos o seguinte fato.

\section{Fato 3.6.}

$$
C(T) \leq 2 \sum_{u \in V}(n \rho(u)+\mathbb{P}) d(T, u, w)
$$

Demonstração.

$$
\begin{aligned}
C(T) & =\sum_{u, v \in V}(\rho(u)+\rho(v)) d(T, u, v) \\
& \leq \sum_{u, v \in V}(\rho(u)+\rho(v))(\boldsymbol{d}(\boldsymbol{T}, \boldsymbol{u}, \boldsymbol{w})+\boldsymbol{d}(\boldsymbol{T}, \boldsymbol{v}, \boldsymbol{w})) \\
& \leq \sum_{\boldsymbol{u} \in \boldsymbol{V}} \sum_{\boldsymbol{v} \in \boldsymbol{V}}(\rho(u)+\rho(v))(d(T, u, w)+d(T, v, w)) \\
& =\sum_{u \in V} \sum_{v \in V}(\rho(u)+\rho(v)) d(T, u, w)
\end{aligned}
$$




$$
\begin{aligned}
& +\sum_{u \in V} \sum_{v \in V}(\rho(u)+\rho(v)) d(T, v, w) \\
= & 2 \sum_{u \in V} \sum_{v \in V}(\rho(u)+\rho(v)) d(T, u, w) \\
= & 2 \sum_{u \in V}(n \rho(u)+\mathbb{P}) d(T, u, w) .
\end{aligned}
$$

Antes de prosseguir precisamos definir os conceitos de centroide e $\rho$-centroide de uma árvore.

Definição 3.7. Dada uma árvore $T$, denotamos o vértice $u \in V_{T}$ como centroide de $T$ se ao eliminar u e as arestas de $T$ incidentes nele, cada componente tem no máximo $\frac{n_{T}}{2}$ vértices. Se cada vértice de $T$ tem um peso $\rho$ associado, então o vértice u de $T$ é denotado $\rho$-centroide se ao eliminar $u$ e as arestas incidentes nele de $T$ temos que a soma dos requerimentos de cada componente é no máximo $\frac{\sum_{u \in V_{T}} \rho(u)}{2}$.

Considere que $T^{*}$ é uma solução ótima do SROCT com instância $I$. Ainda, consideremos $w_{c}$ e $w_{\rho}$ o centroide e o $\rho$-centroide de $T^{*}$, respectivamente, e denotemos por $P$ o caminho em $T^{*}$ entre $w_{c}$ e $w_{\rho}$. Então, provemos o seguinte fato.

Fato 3.8. Para cada aresta $e \in P$, o requerimento que passa por e em $T^{*}$ é no mínimo $\frac{n \mathbb{P}}{2}$.

Demonstração. Denotemos por $T_{1}$ e $T_{2}$ as duas sub-árvores de $T^{*}$ obtidas ao eliminar e de $T^{*}$. Sem perda de generalidade, consideremos que $w_{c} \in V_{T_{2}}$ e $w_{\rho} \in V_{T_{1}}$. Logo, o total de requerimento que passa por $e$ tem valor:

$$
\begin{aligned}
& n_{T_{2}} \sum_{u \in V_{T_{1}}} \rho(u)+n_{T_{1}} \sum_{u \in V_{T_{2}}} \rho(u) \\
= & n_{T_{2}} \sum_{u \in V_{T_{1}}} \rho(u)+\left(n-n_{T_{2}}\right)\left(\mathbb{P}-\sum_{u \in V_{T_{1}}} \rho(u)\right) \\
= & \mathbf{2}\left(\boldsymbol{n}_{\boldsymbol{T}_{\mathbf{2}}}-\frac{\boldsymbol{n}}{\mathbf{2}}\right)\left(\sum_{\boldsymbol{u} \in \boldsymbol{V}_{\boldsymbol{T}_{\mathbf{1}}}} \boldsymbol{\rho}(\boldsymbol{u})-\frac{\mathbb{P}}{\mathbf{2}}\right)+\frac{n \mathbb{P}}{2} .
\end{aligned}
$$

Como $w_{c} \in V_{T_{2}}$ e $w_{\rho} \in V_{T_{1}}$, temos que $n_{T_{2}} \geq \frac{n}{2}$ e que $\sum_{u \in V_{T_{1}}} \rho(u) \geq \frac{\mathbb{P}}{2}$. O que faz com que o primeiro termo da equação acima seja positivo, portanto:

$$
n_{T_{2}} \sum_{u \in V_{T_{1}}} \rho(u)+n_{T_{1}} \sum_{u \in V_{T_{2}}} \rho(u) \geq \frac{n \mathbb{P}}{2} .
$$

Provemos agora o seguinte limitante inferior para o custo de comunicação de $T^{*}$.

Fato 3.9.

$$
C\left(T^{*}\right) \geq \sum_{u \in V}(n \rho(u)+\mathbb{P}) d(T, u, P)+n \mathbb{P} \omega(P)
$$


Demonstração. Considere que $P_{T^{*}}(u, v)$ é o caminho em $T^{*}$ entre os vértices $u$ e $v$ de $G$, e definamos $\overline{P(u)}$ o conjunto de vértices de $V$ cujo caminho até $u$ em $T^{*}$ não tem interseção com o caminho $P$, isto é $\overline{P(u)}=\left\{v \mid P_{T^{*}}(u, v) \cap P=\emptyset\right\}$. Então:

$$
\begin{aligned}
C\left(T^{*}\right)= & \sum_{u, v \in V}(\rho(u)+\rho(v)) d\left(T^{*}, u, v\right) \\
= & \sum_{u, v \in V} \rho(u) d\left(T^{*}, u, v\right) \\
& +\sum_{u, v \in V} \rho(v) d\left(T^{*}, u, v\right) \\
= & 2 \sum_{u, v \in V} \rho(u) d\left(T^{*}, u, v\right) \\
\geq & 2 \sum_{u \in V} \sum_{v \notin \overline{P(u)}} \rho(u)\left(d\left(T^{*}, u, P\right)+d\left(T^{*}, v, P\right)\right) \\
& +2 \sum_{u, v \in V} \rho(u) \omega\left(P_{T^{*}} \cap P\right) .
\end{aligned}
$$

Analisemos a linha (3.1) da equação acima.

$$
\begin{aligned}
& 2 \sum_{u \in V} \sum_{v \notin \overline{P(u)}} \rho(u)\left(d\left(T^{*}, u, P\right)+d\left(T^{*}, v, P\right)\right) \\
= & 2 \sum_{u \in V} \sum_{v \notin \overline{P(u)}} \rho(u) d\left(T^{*}, u, P\right)+2 \sum_{u \in V} \sum_{v \notin \overline{P(u)}} \rho(u) d\left(T^{*}, v, P\right) \\
= & 2 \sum_{u \in V} \boldsymbol{\rho}(\boldsymbol{u}) \boldsymbol{d}\left(\boldsymbol{T}^{*}, \boldsymbol{u}, \boldsymbol{P}\right) \sum_{v \notin \overline{P(u)}} 1+2 \sum_{\boldsymbol{v} \in \boldsymbol{V}} \boldsymbol{d}\left(\boldsymbol{T}^{*}, \boldsymbol{v}, \boldsymbol{P}\right) \sum_{\boldsymbol{u} \notin \overline{\boldsymbol{P}(\boldsymbol{v})}} \rho(u) \\
\geq & 2 \sum_{u \in V} \rho(u) d\left(T^{*}, u, P\right) \frac{\boldsymbol{n}}{\mathbf{2}}+2 \sum_{v \in V} d\left(T^{*}, v, P\right) \frac{\mathbb{P}}{\mathbf{2}} \\
= & \sum_{u \in V}(n \rho(u)+\mathbb{P}) d\left(T^{*}, u, P\right) .
\end{aligned}
$$

Como $P$ é o caminho em $T^{*}$ entre o centroide e o $\rho$-centroide desta árvore, temos que para qualquer vértice $u,|\overline{P(u)}| \leq \frac{n}{2}$ e $\sum_{v \notin \overline{P(u)}} \rho(v) \leq \frac{\mathbb{P}}{2}$. Isto justifica a desigualdade na linha (3.3).

Agora analisemos a linha (3.2).

$$
\begin{aligned}
& 2 \sum_{u, v \in V} \rho(u) \omega\left(P_{T^{*}} \cap P\right) \\
= & 2 \sum_{u, v \in V} \rho(u) \sum_{e \in E_{P_{T^{*} \cap P}}} \omega(e) \\
= & 2 \sum_{e \in E_{P}} \omega(e) \sum_{\boldsymbol{v} \in \boldsymbol{V} \boldsymbol{u} \in \boldsymbol{V} \mid \boldsymbol{e} \in \boldsymbol{E}_{\boldsymbol{P}_{\boldsymbol{T}^{*}}(\boldsymbol{u}, \boldsymbol{v})}} \boldsymbol{\rho}(\boldsymbol{u}) .
\end{aligned}
$$

Observe que o termo em negrito da equação (3.4) representa o requerimento que passa pela aresta $e \in P$ e pelo fato 3.8 esse valor é limitado inferiormente por $\frac{n \mathbb{P}}{2}$. Logo: 


$$
\begin{aligned}
& 2 \sum_{u, v \in V} \rho(u) \omega\left(P_{T^{*}} \cap P\right) \\
\geq & 2 \sum_{e \in E_{P}} \omega(e) \frac{n \mathbb{P}}{2} \\
= & n \mathbb{P} \omega(P) .
\end{aligned}
$$

Usando as equações (3.4) e (3.5) temos:

$$
C\left(T^{*}\right) \geq \sum_{u \in V}(n \rho(u)+\mathbb{P}) d(T, u, P)+n \mathbb{P} \omega(P) .
$$

Considere então as árvores $T_{w_{c}}$ e $T_{w_{\rho}}$ construídas a partir dos caminhos mínimos a todos os vértices de $V$ de $w_{c}$ e $w_{\rho}$, respectivamente.

$\mathrm{O}$ fato 3.6 nos garante:

$$
C\left(T_{w_{c}}\right) \leq 2 \sum_{u \in V}(n \rho(u)+\mathbb{P}) d\left(T_{w_{c}}, u, w_{c}\right)
$$

Como as distâncias em $T_{w_{c}}$ de $w_{c}$ para qualquer outro vértice são mínimas, temos:

$$
C\left(T_{w_{c}}\right) \leq 2 \sum_{u \in V}(n \rho(u)+\mathbb{P}) d\left(T^{*}, u, w_{c}\right)
$$

Similarmente acontece para $T_{w_{\rho}}$ :

$$
C\left(T_{w_{\rho}}\right) \leq 2 \sum_{u \in V}(n \rho(u)+\mathbb{P}) d\left(T^{*}, u, w_{\rho}\right)
$$

Observe que:

$$
\begin{aligned}
& \min \left\{C\left(T_{w_{c}}\right), C\left(T_{w_{\rho}}\right)\right\} \\
\leq & \frac{C\left(T_{w_{c}}\right)}{2}+\frac{C\left(T_{w_{\rho}}\right)}{2} \\
\leq & \sum_{u \in V}(n \rho(u)+\mathbb{P}) d\left(T^{*}, u, w_{c}\right)+\sum_{u \in V}(n \rho(u)+\mathbb{P}) d\left(T^{*}, u, w_{\rho}\right) \\
= & \sum_{u \in V}(n \rho(u)+\mathbb{P})\left(d\left(T^{*}, u, w_{c}\right)+d\left(T^{*}, u, w_{\rho}\right)\right) \\
= & \sum_{u \in V}(n \rho(u)+\mathbb{P})\left(\mathbf{2} d\left(\boldsymbol{T}^{*}, \boldsymbol{u}, \boldsymbol{P}\right)+\boldsymbol{\omega}(\boldsymbol{P})\right) \\
= & 2 \sum_{u \in V}(n \rho(u)+\mathbb{P}) d\left(T^{*}, u, P\right)+\sum_{u \in V}(n \rho(u)+\mathbb{P}) \omega(P) \\
\leq & 2 \sum_{u \in V}(n \rho(u)+\mathbb{P}) d\left(T^{*}, u, P\right)+\mathbf{2 n} \mathbb{P} \omega(P)
\end{aligned}
$$




$$
\leq 2 C\left(T^{*}\right)
$$

Observe que a última desigualdade é dada pelo fato 3.9. Com isto provamos que uma das árvores de distâncias mínimas é uma 2-aproximação.

\subsection{Aproximações para o $p$-OCT}

No caso do p-OCT a primeira proposta de aproximação foi dada por Wu no ano 2002 ([Wu02]) para o caso particular em que $p=2$ e o requerimento entre um fonte e qualquer outro vértice é igual a 1, isto é o 2-MRCT. No artigo, Wu percebe que similarmente ao caso do PROCT, a redução do MRCT para MRCT métrico, apresentada na seção 1.3, também se aplica do 2-MRCT para o 2-MRCT métrico. Ainda, mostra um PTAS para o caso métrico do 2-MRCT com pesos, que também é válido para o 2-MRCT métrico e, portanto, válido para o 2-MRCT com grafos gerais.

No capítulo 4, generalizamos esse resultado mostrando um lema que não só se aplica para qualquer $p$ fixo maior do que 2, mas que também se aplica a outros casos particulares do $p$-OCT mais gerais dos que aqui tratados.

Finalmente, em 2004 ([Wu04]), Wu apresenta uma 2-aproximação para o p-OCT métrico com tempo $O\left(n^{p-1}\right)$. Esta aproximação é baseada em ideias similares às que apresentamos no próximo capítulo para casos particulares do p-OCT. No mesmo artigo, Wu obtém uma 3-aproximação para o 2-OCT em grafos gerais, baseada no algoritmo para reduzir o MRCT ao MRCT métrico. Sendo estes os últimos resultados sobre aproximação encontrados na literatura até o momento para OCT e suas versões. Na próxima seção apresentamos um resumo dos resultados apresentados neste capítulo.

\subsection{Resumo}

Nesta seção mostramos a relação entre os problemas, adicionando na Figura 3.1 as melhores razões de aproximação encontradas e ilustramos em uma tabela estes valores.

\begin{tabular}{|c|c|c|c|}
\hline Problema & \multicolumn{3}{|c|}{ Melhor Resultado na Literatura } \\
\hline & Razão & Complexidade & Referência \\
\hline MRCT & PTAS & $O\left(n^{2\left\lceil\frac{2}{\epsilon}\right\rceil-2}\right)$ & {$\left[\mathrm{WLB}^{+} 98\right]$} \\
\hline 2 -MRCT & 2 & $O\left(n^{\left\lceil\frac{1}{\epsilon}+1\right\rceil}\right)$ & {$[\mathrm{Wu02]}$} \\
\hline $\begin{array}{c}\text { 2-MRCT } \\
\text { com pesos métrico }\end{array}$ & PTAS & $O\left(n^{\left\lceil\frac{1}{\epsilon}+1\right\rceil}\right)$ & {$[\mathrm{Wu02]}$} \\
\hline 2 -OCT & 3 & $O\left(\left|E_{G}\right|+n \log n\right)$ & {$[\mathrm{Wu04]}$} \\
\hline $\begin{array}{c}p \text {-OCT } \\
\text { métrico }\end{array}$ & 2 & $O\left(n^{p-1}\right)$ & {$[\mathrm{Wu04]}$} \\
\hline SROCT & 2 & $O\left(n^{3}\right)$ & {$[\mathrm{WCT00a}]$} \\
\hline PROCT & PTAS & $O\left(k^{k-1} n^{2 k}\left(\frac{q}{\lambda}\right)^{k}\right)$ & {$[\mathrm{WCT00b]}$} \\
\hline
\end{tabular}

Tabela 3.1: Resumo dos resultados de aproximação na literatura. Em todos os PTAS se considera uma $(1+\epsilon)$-aproximação. No caso do PROCT, $\epsilon=\frac{2}{k+1}+\left(\frac{2}{q}+\frac{1}{q^{2}}+\lambda \frac{(k+3)^{3}}{k+1}\right) \frac{k+3}{k+1}$, com $k$ e $q$ inteiros positivos e $\lambda$ um valor positivo menor que 1 . 


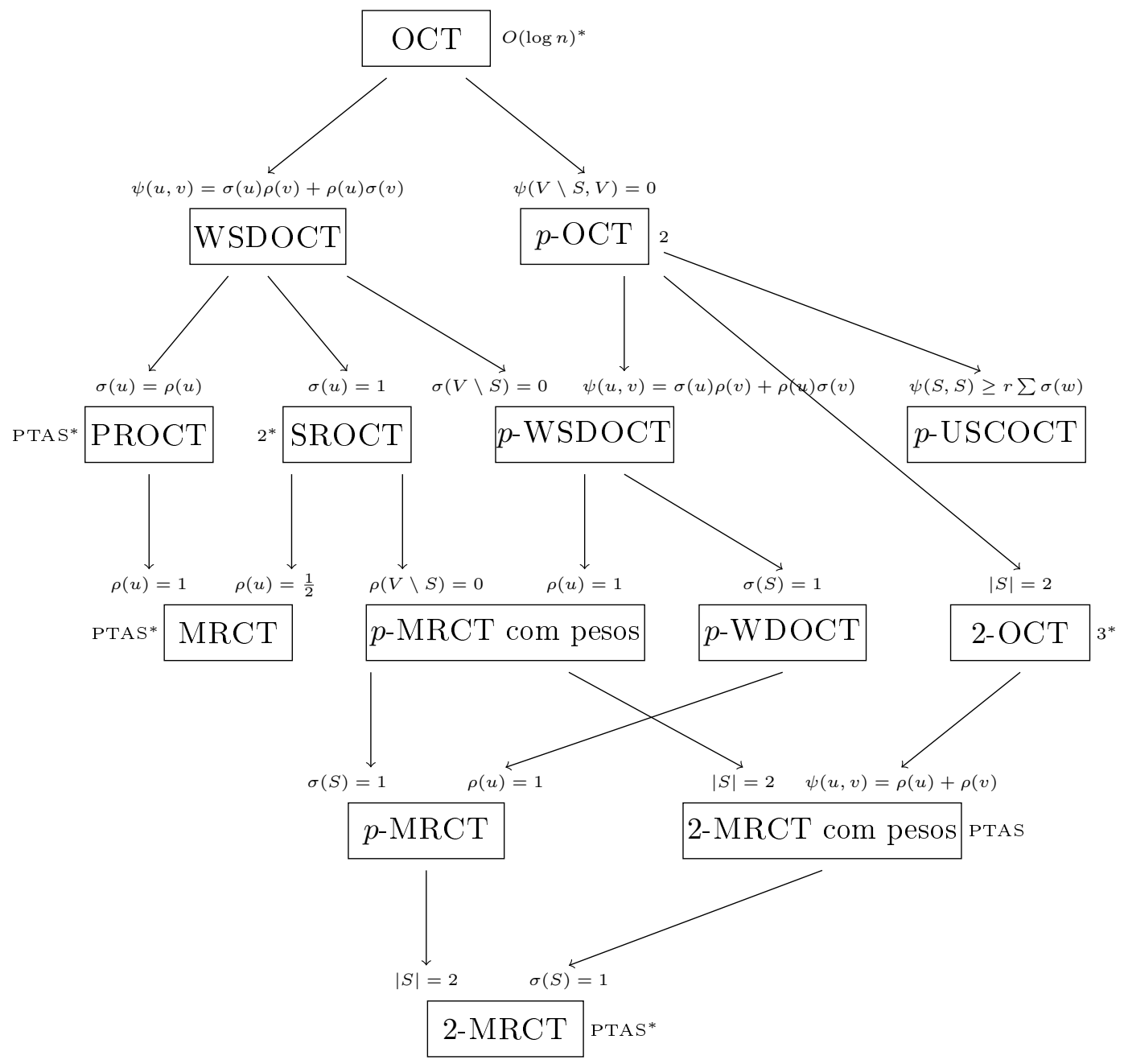

Figura 3.1: Relação entre o OCT e suas variantes NP-dificeis. Do lado de cada problema relacionado, aparece a melhor razão de aproximação na literatura. Aqueles para os quais esse valor não é especificado têm como melhor razão a melhor dentre os problemas que o generalizam. Observe que aqueles valores com * são aproximações que se aplicam tanto ao caso métrico quanto a grafos gerais, as que não tem o símbolo $*$ se aplicam somente ao caso métrico. 


\section{Capítulo 4}

\section{PTASs para variantes do $p$-OCT}

Neste capítulo mostramos como encontrar de forma eficiente um $k$-centro válido ótimo para $k$ fixo. Num lema apresentado a seguir mostramos condições suficientes para diferentes casos particulares do $p$-OCT métrico. Este resultado permite a obtenção de PTAS para diferentes casos particulares do $p$-OCT métrico.

Na Seção 4.1, mostramos um algoritmo polinomial que obtém um $k$-centro ótimo para um $k$ fixo. Na Seção 4.2 demonstramos o lema principal do capítulo. Na Seção 4.3 mostramos como aplicar o resultado e obter PTASs para diferentes variantes do $p$-OCT.

\section{$4.1 \quad k$-centro ótimo}

Primeiro lembramos o conceito de $k$-centro (dado no capítulo anterior) e apresentamos os conceitos de $k$-centro válido e esqueleto, que usamos para aproximar soluções do OCT e $p$-OCT.

Definição 4.1. Dados um grafo $G$ e um inteiro positivo $k$, um $k$-centro de $G$ é uma árvore geradora de $G$ com não mais do que $k$ vértices interiores (isto é, pelo menos $n-k$ folhas). Um esqueleto $\tau$ de um $k$-centro $T$ de $G$ é uma árvore que resulta de eliminar $n-k$ folhas de $T$. $T$ pode ser denotado pela dupla $(\tau, S)$, onde $\tau$ é um esqueleto de $T$ e $S=\left\{S_{u}\right\}_{u \in V_{\tau}}$ é um conjunto cujos elementos são conjuntos de folhas de $T$, e para cada vértice u de $\tau, S_{u}$ contém as folhas adjacentes de u em $T$ (note que $|S|=k$ ).

Definição 4.2. Dados um grafo $G$ e um conjunto $S$ de $p$ vértices de $G$, um k-centro $T$ de $G$ com $k \geq p$ é válido se tem ao menos um esqueleto $\tau$ que contém todos os vértices em S. Neste caso $\tau$ é um esqueleto válido de $T$.

Inicialmente vamos considerar o problema de encontrar um $k$-centro válido ótimo para o $p$ OCT métrico, para um inteiro $k$ fixo. Como veremos a seguir este problema é central nos PTASs propostos.

Problema 4.3. k-Centro Válido Ótimo para o p-OCT Métrico.

Entrada: Um inteiro positivo $k \geq p$ e uma instância do p-OCT métrico: um grafo completo $G$, com função de comprimento não negativa sobre as arestas que satisfaz a desigualdade triangular, $\omega: E \rightarrow \mathbb{Q}_{+}$, um sub-conjunto $S$ de $V$, com $p$ vértices e uma função não negativa de requerimento entre os vértices de $S$ e os de $V, \psi: S \times V \rightarrow \mathbb{Q}_{+}$.

Saída: Um k-centro válido $T$ de $G$ que minimize o custo total de comunicação: 


$$
C(T)=\sum_{u \in S} \sum_{v \in V} \psi(u, v) d(T, u, v) .
$$

Como um esqueleto válido de um $k$-centro deve conter os $p$ vértices de $S$ além de $k-p$ outros vértices, então é possível enumerar os $\left(\begin{array}{c}n \\ k-p\end{array}\right)=O\left(n^{k-p}\right)$ subconjuntos de $k$ vértices que podem definir um esqueleto válido. Para cada sub-conjunto é possível enumerar as $O\left(k^{k}\right)$ possíveis árvores sobre os $k$ vértices do conjunto. Logo, o número de possíveis esqueletos válidos é $O\left(k^{k} n^{k-p}\right)$.

Uma solução do $k$-centro válido ótimo para o $p$-OCT métrico com $k$ fixo é encontrada enumerando os esqueletos válidos, onde para cada esqueleto válido $\tau$ é calculado um $k$-centro ótimo com esqueleto $\tau$, finalmente selecionando o $k$-centro com menor custo de comunicação entre eles. Como observamos acima, para $k$ fixo, o número total de escolhas é polinomial.

Se $\tau$ é um esqueleto válido, então em um $k$-centro $T$ com esqueleto $\tau$ cada vértice em $V_{G}-V_{\tau}$ é adjacente a algum vértice de $\tau$ (isto é, os vértices de $V_{G}-V_{\tau}$ são folhas de $T$ ).

Como todos os vértices de $S$ pertencem a $\tau$, denotando por $u_{v}$ o vértice de $\tau$ adjacente a um vértice $v \in V_{G}-V_{\tau}$ em um $k$-centro válido $T$, tem-se que:

$$
C(T)=C(\tau)+\sum_{v \in V_{G}-V_{\tau}} \sum_{w \in S} \psi(w, v)\left(d\left(\tau, w, u_{v}\right)+\omega\left(u_{v}, v\right)\right) .
$$

Logo, queremos encontrar para cada $v \in V_{G}-V_{\tau}$ o melhor vértice em $\tau$ para ser adjacente de $v$ em um $k$-centro ótimo com esqueleto $\tau$, basta considerar o vértice $u_{v}^{*} \in V_{\tau}$ que minimiza:

$$
\sum_{w \in S} \psi(w, v)\left(d\left(\tau, w, u_{v}\right)+\omega\left(u_{v}, v\right)\right)
$$

Para obter de forma eficiente $u_{v}^{*}$ de cada $v \in V$, primeiro pré-calculamos as distâncias $d(\tau, w, u)$ entre cada $w \in S$ e $u \in V_{\tau}$, o que pode ser feito em tempo $O(|S| k)=O(p k)$. Depois, para cada par de vértices $v \in V_{G}-V_{\tau}$ e $u \in V_{\tau}$ (isto é, um total de $k(n-k)$ pares) calculamos o custo de colocar $v$ como adjacente a $u$ em um $k$-centro, o que pode ser computado em tempo $O(|S|)=O(p)$, se usadas as distâncias pré-calculadas. Assim, é possível obter um $k$-centro ótimo com esqueleto $\tau$ em tempo $O(k(n-k) p+p k)=O(n p k)$.

Portanto, um $k$-centro válido ótimo para $p$-OCT pode ser obtido em $O\left(k^{k+1} n^{k-p+1} p\right)$.

Lema 4.4. Dado um inteiro $k$ fixo, com $k \geq p$, um $k$-centro válido ótimo para $p$-OCT pode ser computado em tempo $O\left(n^{k-p+1}\right)$.

\subsection{Lema de aproximação para variantes métricas do $p$-OCT}

Esta seção apresenta o principal resultado deste capítulo que é um lema com condições suficientes para que um dado caso particular do $p$-OCT métrico admita um PTAS. Portanto a partir deste ponto considere $G$ o grafo de uma instância do $p$-OCT métrico e $S$ seu conjunto de vértices fonte.

Primeiro introduzimos o conceito de $\delta$-caminho-balanceado com $0<\delta \leq \frac{1}{2}$; esta definição está baseada em conceitos similares introduzidos em [WLB $\left.{ }^{+} 98\right]$, [WCT00a] e [WCT00b]. Antes, 
precisamos introduzir alguns conceitos e notações.

Definição 4.5. Dada uma árvore geradora $T$ de $G$, denotamos por $T_{S}$ a sub-árvore minimal de $T$ que contém todos os vértices de $S$. Observe que toda folha de $T_{S}$ é um vértice de $S$, caso contrário $T_{S}$ não seria minimal.

Definição 4.6. Dado $S^{\prime} \subseteq S$ e $U \subseteq V$ definimos:

$$
\psi\left(S^{\prime}, U\right)=\sum_{u \in S^{\prime}} \sum_{v \in U} \psi(u, v) .
$$

Definição 4.7. Dada uma árvore geradora $T$ de $G$ e um caminho $P=w_{1}, \ldots, w_{h}$ de $T$, denotamos por:

- $f_{P}=w_{1}$ e $l_{P}=w_{h}$ os extremos de $P$;

- $V_{P}^{f}$ : o conjunto de vértices de $T$ conectados a $P$ através do vértice $f_{P}$ (incluindo o próprio $\left.f_{P}\right)$;

- $V_{P}^{m}$ : o conjunto de vértices de $T$ conectados a $P$ através de algum vértice interno de $P$ (incluindo os vértices internos de P);

- $V_{P}^{l}$ : o conjunto de vértices de $T$ conectados a $P$ através do vértice $l_{P}$ (incluindo o próprio $l_{P}$ ).

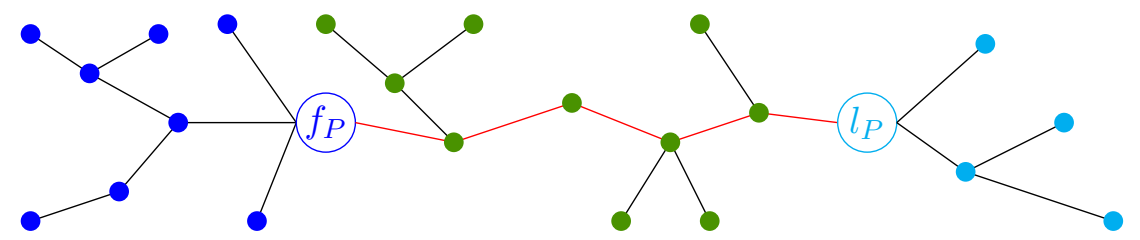

Figura 4.1: Exemplo de $V_{P}^{f}, V_{P}^{m}$ e $V_{P}^{l}$ para o caminho $P$ da árvore $T$. Observe que $P$ é o caminho que conecta os vértices $f_{P}$ e $l_{P}$ em $T, V_{P}^{f}$ é o conjunto de vértices à esquerda de $f_{P}$ (incluindo o próprio $f_{P}$ ), $V_{P}^{l}$ é o conjunto de vértices à direita de $l_{P}$ (incluindo o próprio $l_{P}$ ), e $V_{P}^{m}$ é o conjunto que contém o resto de vértices de $T$ (aqueles que não estão em $V_{P}^{f}$ nem $V_{P}^{l}$ ).

Note que a união dos conjuntos $V_{P}^{f}, V_{P}^{m}$ e $V_{P}^{l}$ resulta no conjunto $V\left(V_{P}^{f} \cup V_{P}^{m} \cup V_{P}^{l}=V\right)$ e que esses conjuntos são disjuntos entre si $\left(V_{P}^{f} \cap V_{P}^{m}=\emptyset, V_{P}^{f} \cap V_{P}^{l}=\emptyset\right.$ e $\left.V_{P}^{m} \cap V_{P}^{l}=\emptyset\right)$.

Também denotamos por $S_{P}^{i}$ o conjunto de vértices $V_{P}^{i} \cap S$, com $i \in\{f, m, l\}$.

Definição 4.8. Dizemos que um caminho $P$ de uma árvore geradora de $G$ é $m$-sem-fonte se $S_{P}^{m}=\emptyset$. Se $P$ é m-sem-fonte e $S_{P}^{f} \neq \emptyset$ e $S_{P}^{l} \neq \emptyset$, então dizemos que $P$ é fontes-conector.

Agora definimos um $\delta$-caminho-balanceado, que é um caminho $m$-sem-fonte $(P)$ de uma árvore geradora de $G$, para o qual o total de requerimento a ser entregue nos vértices em $V_{P}^{m}$ é pequeno, isto é, no máximo uma fração $\delta$ do total de requerimento que atravessa $P$. A seguir damos formalmente esta definição.

Definição 4.9. Dado $0<\delta \leq \frac{1}{2}$ e uma árvore $T$ geradora de $G$, um caminho $m$-sem-fonte $P$ de $T$ é um $\delta$-caminho-balanceado se $\psi\left(S_{P}^{f} \cup S_{P}^{l}, V_{P}^{m}\right)=\psi\left(S, V_{P}^{m}\right) \leq \delta\left(\psi\left(S_{P}^{f}, V_{P}^{l}\right)+\psi\left(S_{P}^{l}, V_{P}^{f}\right)\right)$.

Outra definição que será útil nas nossas demonstrações é a de caminhos internamente disjuntos: 
Definição 4.10. Dados dois caminhos $P=u_{1}, \cdots, u_{r}$ e $Q=v_{1}, \cdots, v_{s}$ de um grafo $G$, eles são internamente disjuntos se a interseção dos seus conjuntos de vértices é vazia ou contém elementos que são extremos dos dois caminhos, isto é: $\left(V_{P} \backslash\left\{u_{1}, u_{r}\right\}\right) \cap V_{Q}=\emptyset$ e $V_{P} \cap\left(V_{Q} \backslash\left\{v_{1}, v_{s}\right\}\right)=\emptyset$.

A seguinte proposição estabelece as bases para o lema principal deste capítulo, pois mostra que dada uma árvore geradora $T$, existe um centro válido cujo custo de comunicação é limitado pelo custo de $T$.

Proposição 4.11. Considere $0<\delta \leq \frac{1}{2}$, uma árvore $T$ geradora de $G$ e um conjunto $Y$ de $\delta$ caminhos-balanceados de $T$, internamente disjuntos dois a dois cuja união resulta em $T_{S}$; então existe um $(|Y|+1)$-centro válido $X$ tal que:

$$
C(X) \leq(1+2 \delta) C(T)
$$

Demonstração. Primeiro, é fácil ver a existência de uma árvore geradora $\bar{T}$ de custo $C(\bar{T}) \leq C(T)$, tal que $\bar{T}_{S}=T_{S}$ e todos os vértices de $\bar{T}$ que não pertencem a $T_{S}$ são folhas. Observe que se todos os vértices de $T$ que não pertencem a $T_{S}$ são folhas, então $\bar{T}=T$; caso contrário existe uma folha $u$ de $T$ que não é adjacente a nenhum vértice de $T_{S}$ e denote por $v$ o elemento em $T_{S}$ mais próximo a $u$. Como todos os vértices de $S$ estão em $T_{S}$ e o grafo é métrico, a árvore $T^{\prime}$ geradora de $G$, obtida ao remover a aresta adjacente a $u$ em $T$ e adicionar a aresta $(v, u)$, satisfaz $C\left(T^{\prime}\right) \leq C(T)$. Repetindo esse processo para cada folha que não é adjacente a um vértice de $T_{S}$ se obtém uma árvore $\bar{T}$ com a propriedade desejada.

O $(|Y|+1)$-centro válido $X$ de $G$ desejado é construído como segue:

- O esqueleto $\tau$ de $X$ tem como conjunto de vértices os extremos dos caminhos em $Y$. Dois vértices $u, v \in \tau$ são adjacentes em $\tau$ se existe um caminho em $Y$ com $u$ e $v$ como extremos. Como os caminhos em $Y$ são internamente disjuntos dois a dois e a união deles resulta em uma árvore $T_{S}$, não é difícil concluir que $\tau$ é uma árvore sobre os extremos dos caminhos de $Y$.

- Para cada $u \in \tau$ e para toda folha $v \in V-\tau$ adjacente a $u$ em $\bar{T}$ é adicionada a aresta $(u, v)$ em $X$.

- Observe que cada vértice $u \in \bar{T}$ que não foi incluído em $X$ pelos passos anteriores pertence ao conjunto $V_{P}^{m}$ de algum caminho $P \in Y$. Logo, o vértice $u$ será colocado adjacente ao mais próximo entre os vértices $f_{P}$ e $l_{P}$, isto é, será adicionada a aresta $\left(u, f_{P}\right)$ em $X$ se $\omega\left(u, f_{P}\right) \leq \omega\left(u, l_{P}\right)$, caso contrário será adicionada a aresta $\left(u, l_{P}\right)$.

Essa construção garante que $X$ seja um $(|Y|+1)$-centro de $G$ com esqueleto $\tau$; logo, é necessário somente analisar o custo de comunicação de $X$.

Vamos calcular o custo total de comunicação da seguinte forma. Para cada aresta analisamos o total de requerimento que passa por ela vezes o seu comprimento. Somando isso para todas as arestas obtemos o custo desejado. Como toda aresta é um caminho com exatamente dois vértices é possível usar nelas a notação introduzida na definição 4.7. Assim:

$$
C(X)=\sum_{e \in E_{X}}\left(\psi\left(S_{e}^{f}, V_{e}^{l}\right)+\psi\left(S_{e}^{l}, V_{e}^{f}\right)\right) \omega(e)
$$




$$
\begin{aligned}
& =\sum_{e \in E_{\tau}}\left(\psi\left(S_{e}^{f}, V_{e}^{l}\right)+\psi\left(S_{e}^{l}, V_{e}^{f}\right)\right) \omega(e) \\
& +\sum_{e \in E_{X-\tau}}\left(\psi\left(S_{e}^{f}, V_{e}^{l}\right)+\psi\left(S_{e}^{l}, V_{e}^{f}\right)\right) \omega(e) .
\end{aligned}
$$

Por construção cada aresta $e \in E_{\tau}$ corresponde a um $\delta$-caminho-balanceado $P \in Y$, tal que:

$$
\psi\left(S_{e}^{f}, V_{e}^{l}\right)+\psi\left(S_{e}^{l}, V_{e}^{f}\right) \leq \psi\left(S_{P}^{f}, V_{P}^{l}\right)+\psi\left(S_{P}^{l}, V_{P}^{f}\right)+\psi\left(S, V_{P}^{m}\right) .
$$

Além disso, lembrando que o grafo é métrico, pela desigualdade triangular tem-se que: $\omega(e) \leq$ $\omega(P), \log$ :

$$
\begin{aligned}
C(X) \leq & \sum_{P \in Y}\left(\psi\left(S_{P}^{f}, V_{P}^{l}\right)+\psi\left(S_{P}^{l}, V_{P}^{f}\right)+\psi\left(S, V_{P}^{m}\right)\right) \omega(P) \\
& +\sum_{e \in E_{X-\tau}}\left(\psi\left(S_{e}^{f}, V_{e}^{l}\right)+\psi\left(S_{e}^{l}, V_{e}^{f}\right)\right) \omega(e) .
\end{aligned}
$$

Como em toda aresta $e \in E_{X-\tau}$ um dos extremos é uma folha fora de $\tau$ e o outro um vértice de $\tau$, denotando por $p(u)$ o vértice de $\tau$ adjacente em $X$ a uma folha $u \in V-\tau$ tem-se:

$$
\begin{aligned}
C(X) \leq & \sum_{P \in Y}\left(\psi\left(S_{P}^{f}, V_{P}^{l}\right)+\psi\left(S_{P}^{l}, V_{P}^{f}\right)+\psi\left(S, V_{P}^{m}\right)\right) \omega(P) \\
& +\sum_{u \in V_{X}-\tau} \psi(S, u) \omega(u, p(u)) \\
\leq & \sum_{P \in Y}\left(\psi\left(S_{P}^{f}, V_{P}^{l}\right)+\psi\left(S_{P}^{l}, V_{P}^{f}\right)+\psi\left(S, V_{P}^{m}\right)\right) \omega(P) \\
& +\sum_{P \in Y} \sum_{u \in V_{P}^{m}} \psi(S, u) \omega(u, p(u))+\sum_{u \in V_{I}} \psi(S, u) \omega(u, p(u)),
\end{aligned}
$$

onde $V_{I}$ é o conjunto de folhas em $X$ que não pertencem a $\tau$ e têm o mesmo adjacente nas árvores $\bar{T}$ e $X$. Note que somente vértices em $V_{P}^{m}$, para algum $P \in Y$, podem estar fora de $V_{I} \cup V_{\tau}$.

Para todo caminho $P \in Y$ e todo vértice $u \in V_{P}^{m}$, o elemento $p(u)$ é um dos extremos de $P$ e satisfaz $\omega(u, p(u))=\min \left\{\omega\left(u, f_{P}\right), \omega\left(u, l_{P}\right)\right\}$, logo:

$$
\begin{aligned}
C(X) \leq & \sum_{P \in Y}\left(\psi\left(S_{P}^{f}, V_{P}^{l}\right)+\psi\left(S_{P}^{l}, V_{P}^{f}\right)+\psi\left(S, V_{P}^{m}\right)\right) \omega(P) \\
& +\sum_{P \in Y} \sum_{u \in V_{P}^{m}} \psi(S, u) \min \left\{\omega\left(u, f_{P}\right), \omega\left(u, l_{P}\right)\right\}+\sum_{u \in V_{I}} \psi(S, u) \omega(u, p(u)) .
\end{aligned}
$$

Se $q(u)$ é o vértice de $T_{S}$ adjacente a $u \in \bar{T}-T_{S}$ e para $u \in T_{S}$ se considera que $q(u)=u$, então:

$$
C(X) \leq \sum_{P \in Y}\left(\psi\left(S_{P}^{f}, V_{P}^{l}\right)+\psi\left(S_{P}^{l}, V_{P}^{f}\right)+\psi\left(S, V_{P}^{m}\right)\right) \omega(P)
$$




$$
+\sum_{P \in Y} \sum_{u \in V_{P}^{m}} \psi(S, u) \min \left\{\omega\left(u, f_{P}\right), \omega\left(u, l_{P}\right)\right\}+\sum_{u \in V_{I}} \psi(S, u) \omega(u, q(u)) .
$$

Pela desigualdade triangular, para todo caminho $P \in Y$ e vértice $u \in V_{P}^{m}$, tem-se que $\omega\left(u, f_{P}\right) \leq$ $\omega\left(q(u), f_{P}\right)+\omega(u, q(u))$ e o mesmo é valido para $l_{P}$. Como $q(u) \in P, \omega\left(q(u), f_{P}\right) \leq \omega(P)$ e $\omega\left(q(u), l_{P}\right) \leq \omega(P)$, é possível concluir:

$$
\min \left\{\omega\left(u, f_{P}\right), \omega\left(u, l_{P}\right)\right\} \leq \omega(P)+\omega(u, q(u)) .
$$

Aplicando a desigualdade acima, obtém-se:

$$
\begin{aligned}
C(X) \leq & \sum_{P \in Y}\left(\psi\left(S_{P}^{f}, V_{P}^{l}\right)+\psi\left(S_{P}^{l}, V_{P}^{f}\right)+\psi\left(S, V_{P}^{m}\right)\right) \omega(P) \\
& +\sum_{P \in Y} \sum_{u \in V_{P}^{m}} \psi(S, u)(\omega(P)+\omega(u, q(u)))+\sum_{u \in V_{I}} \psi(S, u) \omega(u, q(u)) \\
= & \sum_{P \in Y}\left(\psi\left(S_{P}^{f}, V_{P}^{l}\right)+\psi\left(S_{P}^{l}, V_{P}^{f}\right)+\psi\left(S, V_{P}^{m}\right)\right) \omega(P) \\
& +\sum_{P \in Y} \omega(P) \sum_{u \in V_{P}^{m}} \psi(S, u)+\sum_{P \in Y} \sum_{u \in V_{P}^{m}} \psi(S, u) \omega(u, q(u)) \\
& +\sum_{u \in V_{I}} \psi(S, u) \omega(u, q(u)) \\
= & \sum_{P \in Y}\left(\psi\left(S_{P}^{f}, V_{P}^{l}\right)+\psi\left(S_{P}^{l}, V_{P}^{f}\right)+\psi\left(S, V_{P}^{m}\right)\right) \omega(P) \\
& +\sum_{P \in Y} \omega(P) \psi\left(S, V_{P}^{m}\right)+\sum_{u \in V_{\bar{T}-\tau}} \psi(S, u) \omega(u, q(u)) \\
\leq & \sum_{P \in Y}\left(\psi\left(S_{P}^{f}, V_{P}^{l}\right)+\psi\left(S_{P}^{l}, V_{P}^{f}\right)+2 \psi\left(S, V_{P}^{m}\right)\right) \omega(P) \\
& +\sum_{u \in V_{X}-\tau} \psi(S, u) \omega(u, q(u)) .
\end{aligned}
$$

Como para cada vértice $u \in T_{S} \omega(u, q(u))=0$ e todo caminho $P \in Y$ satisfaz $\psi\left(S, V_{P}^{m}\right) \leq$ $\delta\left(\psi\left(S_{P}^{f}, V_{P}^{l}\right)+\psi\left(S_{P}^{l}, V_{P}^{f}\right)\right)$, por ser um $\delta$-caminho-balanceado, então:

$$
\begin{aligned}
C(X) \leq & \sum_{P \in Y}(1+2 \delta)\left(\psi\left(S_{P}^{f}, V_{P}^{l}\right)+\psi\left(S_{P}^{l}, V_{P}^{f}\right)\right) \omega(P) \\
& +\sum_{u \in V_{\bar{T}-T_{S}}} \psi(S, u) \omega(u, q(u)) \\
\leq & (1+2 \delta) C(\bar{T}) \leq(1+2 \delta) C(T) .
\end{aligned}
$$

Do lema acima podemos concluir que dado $0<\delta \leq \frac{1}{2}$, se temos um caso métrico de $p$-OCT para o qual qualquer árvore geradora $T$ de $G$ existe um conjunto de $k \delta$-caminhos-balanceados disjuntos dois a dois cuja união resulta em $T_{S}$, então existe um $(k+1)$-centro $X_{T}$ tal que $C\left(X_{T}\right) \leq$ $(1+2 \delta) C(T)$. Logo, para uma solução ótima $T^{*}$ de uma instância deste problema, existe um $(k+1)$ - 
centro válido que é uma $(1+2 \delta)$-aproximação de $T^{*}$. Portanto, um $(k+1)$-centro válido ótimo é uma $(1+2 \delta)$-aproximação para o problema; e pelo lema 4.4 , é possível encontrar um $(k+1)$-centro válido ótimo em $O\left(n^{k-p+2}\right)$.

Lema 4.12. Se $\Pi$ é um caso particular do p-OCT métrico em que é possivel garantir que para qualquer árvore geradora $T$ de $G$ existem $k \delta$-caminhos-balanceados disjuntos dois a dois cuja união resulta em $T_{S}$, então há uma $(1+2 \delta)$-aproximação para $\Pi$ que pode ser computada em tempo $O\left(n^{k-p+2}\right)$, onde $0<\delta \leq \frac{1}{2}$.

Agora precisamos saber quando um caso particular do OCT métrico se aplica ao lema 4.12, ou seja, precisamos condições suficientes que garantem em um caso métrico do $p$-OCT para qualquer árvore geradora $T$ a existência de um conjunto de $\delta$-caminhos-balanceados internamente disjuntos dois a dois cuja união resulta $T_{S}$. Isso é feito na proposição a seguir.

Proposição 4.13. Dados um caso particular do p-OCT métrico e $0<\delta \leq \frac{1}{2}$, se todo caminho fontes-conector de qualquer árvore $T$ geradora de $G$ pode ser dividido em até $k \delta$-caminhosbalanceados disjuntos dois a dois, então existe um conjunto de $\delta$-caminhos-balanceados disjuntos dois a dois com no máximo $2 k(p-1)$ elementos cuja união resulta $T_{S}$.

Demonstração. Seja $T$ uma árvore geradora de $G$, observe que $T_{S}$ é uma sub-árvore de $T$ com no máximo $p$ folhas; portanto o número de vértices com grau maior que dois em $T_{S}$ é no máximo $p-1$.

Considere $Y$ o conjunto de caminhos obtido a partir de $T_{S}$, de forma tal que um caminho $P$ de $T_{S}$ pertence a $Y$ se, e somente se, cada extremo de $P$ pertence a $S$ ou tem grau diferente de dois em $T_{S}$, e nenhum vértice interno de $P$ está em $S$ ou tem grau diferente de dois em $T_{S}$. Desta forma o conjunto $Y$ tem no máximo $2 p-2$ caminhos (que resulta da soma dos vértices de $S$, que já contém as folhas de $T_{S}$ mais o número de vértices com grau maior que dois em $T_{S}$, menos um). Pela construção $Y$ é um conjunto de caminhos fontes-conectores internamente disjuntos dois a dois, logo cada elemento de $Y$ pode ser dividido em até $k \delta$-caminhos-balanceados internamente disjuntos dois a dois. Portanto, é possível obter um conjunto com não mais do que $2 k(p-1) \delta$-caminhosbalanceados disjuntos dois a dois cuja união resulta $T_{S}$.

Finalmente, usando os resultados do lema 4.12 e da proposição 4.13 pode-se concluir que:

Lema 4.14. Dados $\Pi$ um caso particular do p-OCT métrico e $0<\delta \leq \frac{1}{2}$, se para todo caminho fontes-conector $P$ de qualquer árvore $T$ geradora de $G$ existem até $k \delta$-caminhos-balanceados internamente disjuntos cuja união resulta $P$, então existe uma $(1+2 \delta)$-aproximação para $\Pi$ em tempo $O\left(n^{(2 k-1)(p-1)+1}\right)$.

Na próxima seção exemplificamos três casos do OCT métrico em que a proposição se aplica.

\subsection{PTASs para três casos particulares do p-OCT métrico}

Na seção anterior foi apresentado um resultado que permite obter boas aproximações para casos particulares do p-OCT métrico que satisfaçam certas condições. Esta seção ilustra como aplicar esse resultado em três variantes NP-difíceis do $p$-OCT métrico. 


\subsubsection{PTAS para o $p$-USCOCT métrico}

O primeiro problema em que aplicaremos o lema 4.14 é o $p$-USCOCT métrico.

Assim, provaremos que dado $0<\delta \leq \frac{1}{2}$, para toda instância do $p$-USCOCT métrico é possível dividir qualquer caminho fonte-conector $P$ de qualquer árvore geradora de $G$ em não mais do que $2 \log _{\frac{1}{1+\delta}}(r) \delta$-caminhos-balanceados internamente disjuntos. Isto implica que ao aplicar o lema 4.14 se obtém:

Teorema 4.15. Existe um PTAS para o p-USCOCT métrico em que dado $0<\delta \leq \frac{1}{2}$, o algoritmo garante uma $(1+2 \delta)$-aproximação em tempo $O\left(n\left(4 \log _{\frac{1}{1+\delta}}(r)-1\right)(p-1)+1\right)$.

Demonstração. Considere um caminho fonte-conetor $P$ de uma árvore geradora de $G$. Primeiro analisamos o requerimento que vai de $S_{P}^{f}$ para $V_{P}^{m} \cup V_{P}^{l}$.

Defina a partir de $P$ um inteiro $k$ e um conjunto $Q=\left\{P_{i}\right\}_{i=1}^{k}$ tal que $f_{P_{1}}=f_{P}, l_{P_{k}}=l_{P}$, $l_{P_{i}}=f_{P_{i+1}}(i<k)$ e para cada $i$ :

$$
\psi\left(S_{P}^{f}, V_{P_{i}}^{m}\right) \leq \delta\left(\psi\left(S_{P}^{f}, V_{P}^{l}\right)+\sum_{j=i+1}^{k} \psi\left(S_{P}^{f}, V_{P_{j}}^{f} \cup V_{P_{j}}^{m}\right)\right) .
$$

Desta forma $Q$ é um conjunto com $k$ caminhos internamente disjuntos dois a dois cuja união resulta $P$. Além disso, o primeiro caminho de $P_{1}$ satisfaz:

$$
\begin{aligned}
\psi\left(S_{P}^{f}, V_{P_{1}}^{m}\right) & \leq \delta\left(\psi\left(S_{P}^{f}, V_{P}^{m} \cup V_{P}^{l}\right)-\psi\left(S_{P}^{f}, V_{P_{1}}^{m}\right)\right) \\
& \leq \frac{\delta}{1+\delta} \psi\left(S_{P}^{f}, V_{P}^{m} \cup V_{P}^{l}\right) .
\end{aligned}
$$

Logo, uma solução gulosa constrói $P_{1}$ selecionando como $l_{P_{1}}$ o último vértice de $P$, o que garante a desigualdade acima. Assim o caminho $P_{1}$ resultante satisfaz:

$$
\psi\left(S_{P}^{f}, V_{P_{1}}^{m} \cup V_{P_{1}}^{l}\right) \geq \frac{\delta}{1+\delta} \psi\left(S_{P}^{f}, V_{P}^{m} \cup V_{P}^{l}\right) .
$$

Observe que se $P_{1} \neq P$, então $P-P_{1}$ é também um caminho fonte-conector. Portanto $Q$ pode ser construído pela união do conjunto $\left\{P_{1}\right\}$ com o resultado de aplicar de forma recursiva o mesmo critério guloso sobre $P-P_{1}$. Logo, uma função para delimitar o inteiro $k=|Q|$ poderia ser:

$$
\begin{aligned}
k(\Psi) & \leq k\left(\Psi-\frac{\delta}{1+\delta} \Psi\right)+1 \quad \text { se } \Psi>\psi\left(S_{P}^{f}, S_{P}^{l}\right) \\
& =k\left(\frac{1}{1+\delta} \Psi\right)+1 \\
k(\Psi) & \leq 1 \quad \text { se } \Psi \leq \psi\left(S_{P}^{f}, S_{P}^{l}\right) .
\end{aligned}
$$

Onde $\Psi=\psi\left(S_{P}^{f}, V_{P}^{m} \cup V_{P}^{l}\right)$.

Portanto, é suficiente que $k$ satisfaça: 


$$
\begin{aligned}
\left(\frac{1}{1+\delta}\right)^{k} \psi\left(S_{P}^{f}, V_{P}^{m} \cup V_{P}^{l}\right) & \leq \psi\left(S_{P}^{f}, S_{P}^{l}\right) \\
\left(\frac{1}{1+\delta}\right)^{k} & \leq \frac{\psi\left(S_{P}^{f}, S_{P}^{l}\right)}{\psi\left(S_{P}^{f}, V_{P}^{m} \cup V_{P}^{l}\right)}
\end{aligned}
$$

Observe que $\sum_{u \in S} \sum_{v \in V} \psi(u, v) \geq \psi\left(S_{P}^{f}, V_{P}^{m} \cup V_{P}^{l}\right)$, o que implica:

$$
\frac{\psi\left(S_{P}^{f}, S_{P}^{l}\right)}{\sum_{u \in S} \sum_{v \in V} \psi(u, v)} \leq \frac{\psi\left(S_{P}^{f}, S_{P}^{l}\right)}{\psi\left(S_{P}^{f}, V_{P}^{m} \cup V_{P}^{l}\right)} .
$$

Assim, encontrando um $k$ que satisfaz:

$$
\left(\frac{1}{1+\delta}\right)^{k} \leq \frac{\psi\left(S_{P}^{f}, S_{P}^{l}\right)}{\sum_{u \in S} \sum_{v \in V} \psi(u, v)}
$$

Também se garante:

$$
\left(\frac{1}{1+\delta}\right)^{k} \leq \frac{\psi\left(S_{P}^{f}, S_{P}^{l}\right)}{\sum_{u \in S} \sum_{v \in V} \psi(u, v)} \leq \frac{\psi\left(S_{P}^{f}, S_{P}^{l}\right)}{\psi\left(S_{P}^{f}, V_{P}^{m} \cup V_{P}^{l}\right)}
$$

Como $P$ é um caminho fonte-conector, temos que $S_{P}^{f}$ e $S_{P}^{l}$ são não vazios, logo tome $u^{f} \in S_{P}^{f}$ e $u^{l} \in S_{P}^{l}$. Observe que $r \leq \frac{\psi\left(u^{f}, u^{l}\right)}{\sum_{u \in S} \sum_{v \in V} \psi(u, v)} \leq \frac{\psi\left(S_{P}^{f}, S_{P}^{l}\right)}{\sum_{u \in S} \sum_{v \in V} \psi(u, v)}$. Um valor de $k$ que satisfaz $\left(\frac{1}{1+\delta}\right)^{k} \leq r$, também satisfaz a expressão anterior, sendo suficiente um valor de $k \geq \log _{\frac{1}{1+\delta}}(r)$.

Uma análise simétrica pode ser feita para o requerimento que vai de $S_{P}^{l}$ para $V_{P}^{m} \cup V_{P}^{f}$, obtendo o correspondente conjunto $Q^{\prime}$. Dessa forma, dividindo $P$ nos vértices que são extremos dos caminhos em $Q \cup Q^{\prime}$, se obtém um conjunto de $\delta$-caminhos balanceados internamente disjuntos dois a dois cuja união é $P$ e o número desses caminhos não é maior do que $2 \log _{\frac{1}{1+\delta}}(r)$.

Concluímos que todo caminho fonte-conector de qualquer árvore geradora de $G$ pode ser dividido em até $2 \log _{\frac{1}{1+\delta}}(r) \delta$-caminhos-balanceados internamente disjuntos dois a dois, e usando o lema 4.14 se chega que para o $p$-USCOCT métrico existe uma $(1+2 \delta)$-aproximação com complexidade de $O\left(n^{\left(4 \log _{\frac{1}{1+\delta}}(r)-1\right)(p-1)+1}\right)$.

\subsection{2 $p$-WSDOCT e $p$-WDOCT métricos}

O próximo problema que analisaremos é o $p$-WSDOCT métrico com parâmetro fixo. A seguir enunciamos o resultado que demonstraremos para este problema fazendo uso do lema 4.14.

Teorema 4.16. Existe um PTAS para o p-WSDOCT métrico com parâmetro fixo, tal que, dado $0<\delta \leq \frac{1}{2}$, o algoritmo encontra, em tempo $O\left(n^{\left(2 \frac{1}{\delta-\delta^{2}}-1\right)(p-1)+1}\right)$, uma $(1+2 \xi \delta)$-aproximação do ótimo onde $\xi=\frac{\sum_{u \in S} \sigma(u)}{\min \{\sigma(u)\}_{u \in S}}$ é o parâmetro fixo.

Demonstração. Para provar o resultado considere um caminho fontes-conector $P$ de qualquer árvore geradora $T$ de $G$ que satisfaça a seguinte condição: 


$$
\sum_{u \in V_{P}^{m}} \rho(u) \leq \delta(1-\delta) \sum_{u \in V} \rho(u)
$$

Observe que:

$$
\begin{aligned}
\psi\left(S, V_{P}^{m}\right) & =\left(\sum_{u \in S} \sigma(u)\right)\left(\sum_{u \in V_{P}^{m}} \rho(u)\right) \leq\left(\sum_{u \in S} \sigma(u)\right) \delta(1-\delta) \sum_{u \in V} \rho(u) \\
& \leq\left(\sum_{u \in S} \sigma(u)\right) \delta\left(1-\left(\delta-\delta^{2}\right)\right) \sum_{u \in V} \rho(u) \\
& =\left(\sum_{u \in S} \sigma(u)\right) \delta\left(\sum_{u \in V} \rho(u)-\left(\delta-\delta^{2}\right) \sum_{u \in V} \rho(u)\right) \\
& \leq\left(\sum_{u \in S} \sigma(u)\right) \delta\left(\sum_{u \in V} \rho(u)-\sum_{u \in V_{P}^{m}} \rho(u)\right) .
\end{aligned}
$$

Como $V=V_{P}^{l} \cup V_{P}^{f} \cup V_{P}^{m}$ :

$$
\begin{aligned}
\psi\left(S, V_{P}^{m}\right) \leq & \left(\sum_{u \in S} \sigma(u)\right) \delta\left(\sum_{u \in V_{P}^{l}} \rho(u)+\sum_{u \in V_{P}^{f}} \rho(u)\right) \\
\leq & \left(\sum_{u \in S} \sigma(u)\right) \delta \frac{\sum_{u \in S_{P}^{f}} \sigma(u)}{\min \{\sigma(u)\}_{u \in S}} \sum_{u \in V_{P}^{l}} \rho(u) \\
& +\left(\sum_{u \in S} \sigma(u)\right) \delta \frac{\sum_{u \in S_{P}^{l}} \sigma(u)}{\min \{\sigma(u)\}_{u \in S}} \sum_{u \in V_{P}^{f}} \rho(u) \\
= & \frac{\sum_{u \in S} \sigma(u)}{\min \{\sigma(u)\}_{u \in S}} \delta\left(\psi\left(S_{P}^{f}, V_{P}^{l}\right)+\psi\left(S_{P}^{l}, V_{P}^{f}\right)\right) .
\end{aligned}
$$

Logo $P$ é um $\frac{\sum_{u \in S} \sigma(u)}{\min \{\sigma(u)\}_{u \in S}} \delta$-caminho-balanceado.

Observe que qualquer caminho fontes-conector de $T$ pode ser dividido em até $\frac{1}{\delta-\delta^{2}}$ caminhos fontes-conectores internamente disjuntos dois a dois que satisfazem:

$$
\sum_{u \in V_{P}^{m}} \rho(u) \leq \delta(1-\delta) \sum_{u \in V} \rho(u)
$$

Portanto, todo caminho fonte-conector de qualquer árvore geradora de $G$ pode ser dividido em até $\frac{1}{\delta-\delta^{2}} \frac{\sum_{u \in S} \sigma(u)}{\min \{\sigma(u)\}_{u \in S}} \delta$-caminhos-balanceados internamente disjuntos dois a dois e aplicando o lema 4.14 é possível concluir que para o $p$-WSDOCT métrico existe uma $\left(1+2 \frac{\sum_{u \in S} \sigma(u)}{\min \{\sigma(u)\}_{u \in S}} \delta\right)$ aproximação com tempo $O\left(n\left(2 \frac{1}{\delta-\delta^{2}}-1\right)(p-1)+1\right)$.

Como visto em capítulos anteriores, o $p$-WDOCT é o caso particular do $p$-WSDOCT onde o requerimento de envio de todos os vértices em $S$ é unitário (isto é, $\sigma(S)=1$ ). Logo, para este 
problema:

$$
\xi=\frac{\sum_{u \in S} \sigma(u)}{\min \{\sigma(u)\}_{u \in S}}=\frac{p}{1}=p .
$$

Como $p$ é fixo, temos que o $p$-WDOCT é um caso particular do $p$-WSDOCT com parâmetro fixo, o que permite usar o teorema 4.16 e enunciar o seguinte resultado para o $p$-WDOCT métrico:

Corolário 4.17. Existe um PTAS para o p-WDOCTmétrico que, dado $0<\delta \leq \frac{1}{2}$, pode encontrar uma $(1+2 p \delta)$-aproximação em tempo $O\left(n^{\left(2 \frac{1}{\delta-\delta^{2}}-1\right)(p-1)+1}\right)$.

\subsection{Resumo}

De forma similar aos capítulos anteriores resumimos as contribuições deste capítulo em uma figura que relaciona os diferentes problemas tratados e as razões de aproximação obtidas, e uma tabela comparativa com os melhores resultados na literatura para estes problemas. ${ }^{1}$

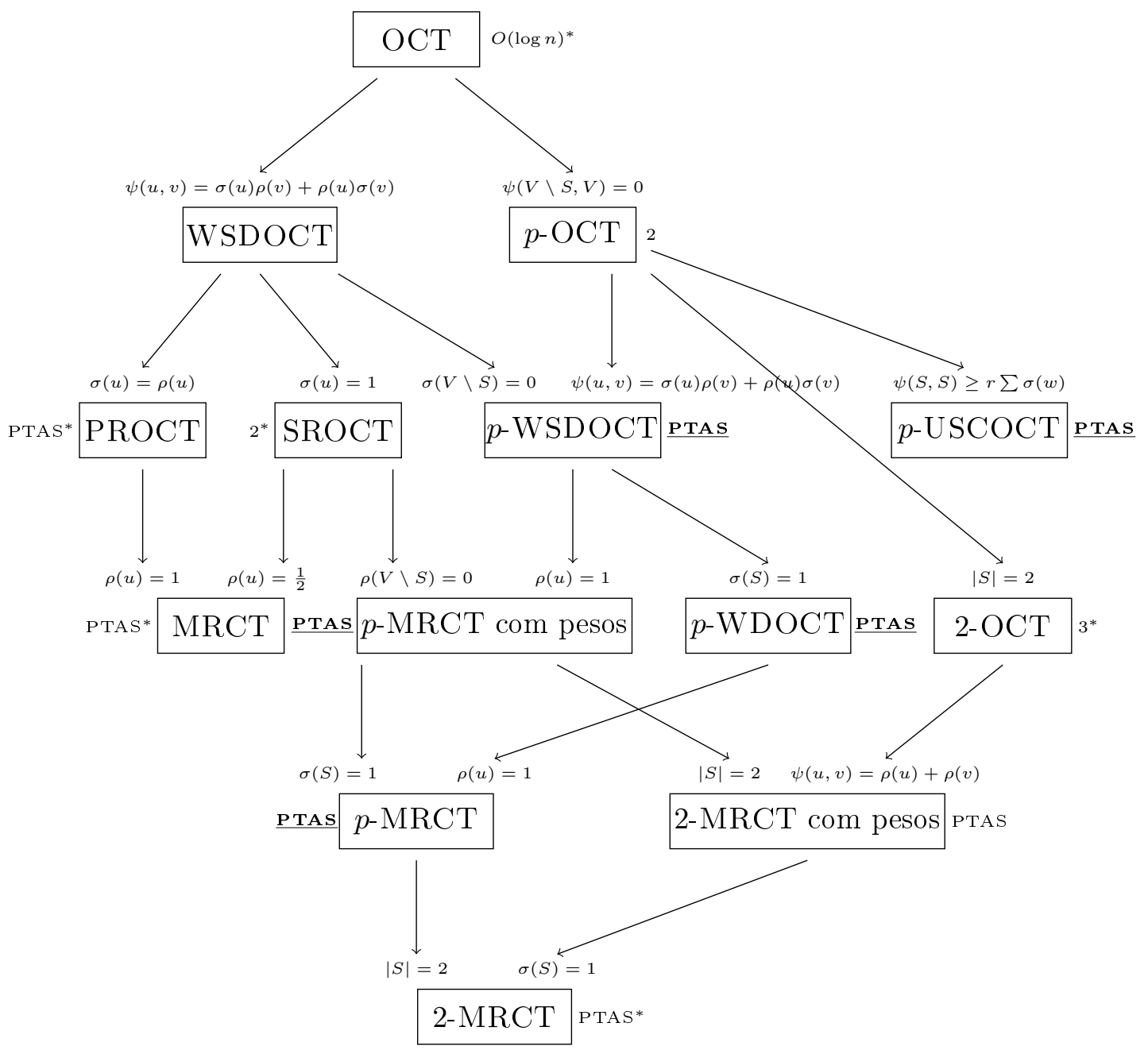

Figura 4.2: Relação entre o OCT e suas variantes NP-difíceis. As aproximações dadas por nós neste capitulo aparecem em negrito e sublinhados.

\footnotetext{
${ }^{1}$ Note que esta análise e comparações consideram os resultados para as versões métricas dos problemas na literatura.
} 


\begin{tabular}{|c|c|c|c|c|c|}
\hline Problema & \multicolumn{3}{|c|}{ Melhor Resultado na Literatura } & \multicolumn{2}{|r|}{ Resultado deste Capítulo } \\
\hline & Razão & Complexidade & Referência & Razão & Complexidade \\
\hline 2-MRCT & PTAS & $\mathbf{O}\left(\mathbf{n}^{\frac{1}{\epsilon}+1}\right)$ & [Wu02] & PTAS & $O\left(n^{\frac{8}{\epsilon(2-\epsilon)}}\right)$ \\
\hline$p$-MRCT & 2 & $O\left(n^{3}\right)$ & [WCT00a] & PTAS & $O\left(n\left(\frac{8 p^{2}}{\epsilon(2 p-\epsilon)}-1\right)(p-1)+1\right)$ \\
\hline $\begin{array}{l}p \text {-MRCT } \\
\text { com pesos }\end{array}$ & 2 & $O\left(n^{3}\right)$ & [WCT00a] & PTAS & $\left.\left.\frac{8 \xi^{2}}{(2 \xi-\epsilon)}-1\right)(p-1)+1\right)$ \\
\hline$p$-WDOCT & 2 & $O\left(n^{p-1}\right)$ & [Wu04] & PTAS & $O\left(n\left(\frac{8 p^{2}}{\epsilon(2 p-\epsilon)}-1\right)(p-1)+1\right)$ \\
\hline $\begin{array}{c}p \text {-WSDOCT } \\
\text { com parâmetro fixo }\end{array}$ & 2 & $O\left(n^{p-1}\right)$ & [Wu04] & PTAS & $O\left(n\left(\frac{8 \xi^{2}}{\epsilon(2 \xi-\epsilon)}-1\right)(p-1)+1\right)$ \\
\hline$p$-USCOCT & 2 & $O\left(n^{p-1}\right)$ & [Wu04] & PTAS & $O\left(n{ }^{\left(4 \log \frac{2}{2+\epsilon}\right.}(r)-1\right)(p-1)+1$ \\
\hline
\end{tabular}

Tabela 4.1: Comparação dos resultado aqui apresentados com os melhores resultados conhecidos anteriormente na literatura. Como pode ser visto, para a maioria dos problemas a solução proposta melhora a razão de aproximação, somente no caso do 2-MRCT a solução não é melhorada (obtendo a mesma razão, porém com uma complexidade maior). Em todos os $\mathrm{PTAS} s$ desta tabela se considera uma aproximação $(1+\epsilon)$ com $\epsilon \in(0,1]$. 


\section{Capítulo 5}

\section{Redução do WSDOCT métrico ao problema do $k$-centro ótimo}

Neste capítulo consideramos o caso métrico do WSDOCT, apresentando uma redução dele ao problema de encontrar um $k$-centro ótimo para o mesmo.

Como mencionado em capítulos anteriores o $p$-WSDOCT é o caso particular do WSDOCT em que dado o sub-conjunto $S$ de vértices de $G$, a função de requerimento de envio é igual a zero para os vértices fora de $S$, isto é, $\sigma: V \backslash S \rightarrow 0$. Também já mencionamos que o SROCT e o PROCT são casos particulares deste problema. O SROCT é o caso em que a função de envio é unitária para todos os vértices de $G$, isto é $\sigma: V \rightarrow 1$; enquanto o PROCT é o caso em as funções de envio e requerimento são iguais, isto é, para cada $u \in V \sigma(u)=\rho(u)$. Logo, um PTAS para o mesmo generalizaria os resultados na literatura para seus diferentes casos particulares.

Nas próximas seções consideramos um $0<\delta \leq \frac{1}{2}$, e provamos que existe um $k$-centro, com $k$ em função de $\delta$, que resulta em uma $\frac{1}{1-\delta}$-aproximação para o WSDOCT métrico. Para isso, de agora em diante, consideramos $I$ uma instância do WSDOCT métrico.

A prova que vamos apresentar é uma generalização não trivial dos resultados apresentados em [WLB ${ }^{+98}$ ], [WCT00a] e [WCT00b]. Nestes artigos, dada uma árvore geradora $T$ de $G$ demonstra-se a existência de um conjunto $Y$ de caminhos internamente disjuntos dois a dois cuja união resulta em uma sub-árvore $S$ de $T$ tal que o custo de comunicação de cada componente $B \in T \backslash S$ é, no máximo, uma pequena fração do custo de comunicação de $T$. Isso implica que a maior parte da comunicação de $T$ passa por $S$. Também é demonstrado que o número de elementos de $Y$ é limitado por uma função de $\delta$, ilustrando como construir um $k$-centro a partir de $Y$, onde $k$ depende da cardinalidade de $Y$. Ainda prova-se que o custo de comunicação deste $k$-centro aproxima o custo de comunicação de $T$ por um fator de $\frac{1}{1-\delta}$.

\subsection{Definições e notações}

Antes de prosseguir, serão apresentadas algumas definições e notações úteis para realizar as provas, tais definições são generalizações das apresentadas em [WLB+98], [WCT00a] e [WCT00b].

Definição 5.1. Denotamos por $\mathbb{S}$ a soma dos requerimentos de envio de todos os vértices de $G$, e 
por $\mathbb{P}$ a soma dos requerimentos de recepção:

$$
\mathbb{S}=\sum_{u \in V} \sigma(u) \quad e \quad \mathbb{P}=\sum_{u \in V} \rho(u)
$$

Definição 5.2. Dado um sub-grafo $H$ de $G$, denotamos por $\sigma(H)$ a soma dos requerimentos de envio dos vértices de $H$ e por $\rho(H)$ a soma dos requerimentos de recepção dos vértices de $H$. Quando $G$ é implícito pelo contexto $\sigma(G)=\mathbb{S}$ e $\rho(G)=\mathbb{P}$.

Definição 5.3. Dados uma árvore geradora $T$ de $G$, um conjunto $H$ de arestas de $T$ e um vértice $u$ de $T$, se denota por $V(T, H, u)$ ao conjunto de vértices que estão na mesma componente que contém u em $T \backslash H$.

A seguinte definição é parecida à apresentada no capítulo anterior para as variantes do $p$-OCT, porém para as demonstrações que seguem precisamos notações mais específicas.

Definição 5.4. Dados uma árvore geradora $T$ de $G$ e um caminho $P=u_{1}, \cdots, u_{h}$ de $T$, denotamos por $f_{P}$ e $l_{P}$, o primeiro e último vértices de $P$ (isto é, $f_{P}=u_{1}$ e $l_{P}=u_{h}$ ). Quando $P$ é implícito pelo contexto $f_{P}=f$ e $l_{P}=l$.

Para os requerimentos de envio definimos:

- $\sigma_{P}^{f}=\sigma\left(V\left(T, E_{P}, f\right)\right)$ é a soma dos requerimentos de envio dos vértices que pertencem à mesma componente em $T-E_{P}$ que contém o primeiro vértice de $P$,

- $\sigma_{P}^{l}=\sigma\left(V\left(T, E_{P}, l\right)\right)$ é a soma dos requerimentos de envio dos vértices que pertencem à mesma componente em $T-E_{P}$ que contém o último vértice de $P$,

- $\sigma_{P}^{m}=\mathbb{S}-\sigma_{P}^{f}-\sigma_{P}^{l}$ é a soma dos requerimentos de envio dos vértices que pertencem a alguma componente de $T-E_{P}$ que contém algum vértice interno de $P$,

- $\mathbb{S}(P, v)=\sum_{i=2}^{h-1} \sigma\left(V\left(T, E_{P}, u_{i}\right)\right) d\left(P, v, u_{i}\right)$ é a soma sobre cada vértice $u_{i}$ interno de $P$ da distância em $P$ de $u_{i}$ até $v$ vezes a soma dos requerimentos de envio da componente de $T-E_{P}$ que contém $u_{i}$,

- $\mathbb{S}_{s}^{f}(P)=\mathbb{S}(P, f), \mathbb{S}_{s}^{l}(P)=\mathbb{S}(P, l)$, representam as somas sobre cada vértice interno de $P$ dos requerimentos de envio dos vértices que pertencem à mesma componente que $u_{i}$ em $T-E_{P}$ vezes a distância de $u_{i}$ até o primeiro (último, respectivamente) vértice de $P$,

- $\sigma_{P}^{\max }=\max \left\{\sigma_{P}^{f}, \sigma_{P}^{l}\right\}, \sigma_{P}^{\min }=\min \left\{\sigma_{P}^{f}, \sigma_{P}^{l}\right\}$,

- Se $\sigma_{P}^{f}=\sigma_{P}^{\max }$ então $\mathbb{S}(P)=\mathbb{S}^{f}(P)$, caso contrário $\mathbb{S}(P)=\mathbb{S}^{l}(P)$.

Notações análogas são feitas para os requerimentos de recepção:

- $\rho_{P}^{f}=\rho\left(V\left(T, E_{P}, f\right)\right), \rho_{P}^{l}=\rho\left(V\left(T, E_{P}, l\right), \rho_{P}^{m}=\mathbb{P}-\rho_{P}^{f}-\rho_{P}^{l}\right.$,

- $\mathbb{P}(P, v)=\sum_{i=2}^{h-1} \rho\left(V\left(T, E_{P}, u_{i}\right)\right) d\left(P, v, u_{i}\right), \mathbb{P}^{f}(P)=\mathbb{P}(P, f), \mathbb{P}^{l}(P)=\mathbb{P}(P, l)$,

- $\rho_{P}^{\max }=\max \left\{\rho_{P}^{f}, \rho_{P}^{l}\right\}, \rho_{P}^{\min }=\min \left\{\rho_{P}^{f}, \rho_{P}^{l}\right\}$,

- Se $\rho_{P}^{f}=\rho_{P}^{\max }$ então $\mathbb{P}(P)=\mathbb{P}^{f}(P)$, caso contrário $\mathbb{P}(P)=\mathbb{P}^{l}(P)$. 


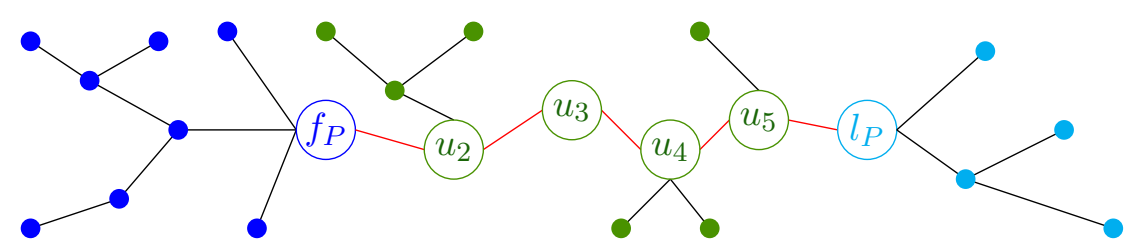

Figura 5.1: Considere a árvore geradora $T$ de $G$ com a mesma função de envio para todos os vértices, onde todas as arestas têm comprimento unitário e $P$ é o caminho em $T$ do vértice $f_{P}$ até o $l_{P}$. Observe que $V\left(T, E_{P}, f_{P}\right)$ é o conjunto de vértices à esquerda de $f_{P}$ (incluindo o próprio $\left.f_{P}\right), V\left(T, E_{P}, l_{P}\right)$ é o conjunto de vértices à direita de $l_{P}$ (incluindo o próprio $\left.l_{P}\right), V\left(T, E_{P}, u_{2}\right)$ é o conjunto de vértices contendo $u_{2}$ e os vértices acima dele, $V\left(T, E_{P}, u_{3}\right)=\left\{u_{3}\right\}, V\left(T, E_{P}, u_{4}\right)$ é o conjunto de vértices contendo $u_{4}$ e os vértice abaixo dele, e $V\left(T, E_{P}, u_{5}\right)$ é o conjunto de vértices contendo $u_{5}$ e o vértice acima dele. Logo, $\sigma_{P}^{f}=9, \sigma_{P}^{l}=5$ e $\sigma_{P}^{m}=10$, o que implica que $\sigma_{P}^{\max }=9$ e $\sigma_{P}^{\min }=5$. Além, $\mathbb{S}^{f}(P)=\sigma\left(V\left(T, E_{P}, u_{2}\right)\right) \times 1+\sigma\left(V\left(T, E_{P}, u_{3}\right)\right) \times$ $2+\sigma\left(V\left(T, E_{P}, u_{4}\right)\right) \times 3+\sigma\left(V\left(T, E_{P}, u_{5}\right)\right) \times 4=4 \times 1+1 \times 2+3 \times 3+2 \times 4=23 e \mathbb{S}^{l}(P)=\sigma\left(V\left(T, E_{P}, u_{2}\right)\right) \times$ $4+\sigma\left(V\left(T, E_{P}, u_{3}\right)\right) \times 3+\sigma\left(V\left(T, E_{P}, u_{4}\right)\right) \times 2+\sigma\left(V\left(T, E_{P}, u_{5}\right)\right) \times 1=4 \times 4+1 \times 3+3 \times 2+2 \times 1=27$, implicando que $\mathbb{S}(p)=23$.

\section{(A Figura 5.1 dá um exemplo desta notação. ${ }^{1}$ )}

Agora é introduzida a ideia de separadores. Um $\delta$-separador é uma sub-árvore de uma árvore geradora $T$ de $G$ cuja remoção resulta em componentes com requerimentos (de envio, recepção ou ambos) limitados por um fator $\delta$ dos valores totais $(\mathbb{S}$ ou $\mathbb{P}$ ). Formalmente:

Definição 5.5. Dados $0<\delta \leq \frac{1}{2}$ e uma árvore geradora $T$ de $G$, uma sub-árvore $S$ de $T$ é um $\delta$ - $\sigma$-separador de $T$ se toda componente $B$ de $T-S$, satisfaz $\sigma(B) \leq \delta \mathbb{S}$. Se toda componente $B$ de $T-S$, satisfaz $\rho(B) \leq \delta \mathbb{P}$, então $S$ é um $\delta$ - $\rho$-separador de $T$; e se ambas as condições são válidas, então $S$ é um $\delta$ - $\sigma \rho$-separador de $T$.

Outras definições que serão úteis são as de $\delta$ - $\sigma \rho$-caminho e $\delta$ - $\sigma \rho$-spine:

Definição 5.6. Dados $0<\delta \leq \frac{1}{2}$ e uma árvore $T$ geradora de $G$, um caminho $P$ de $T$ é um $\delta$ - $\sigma \rho$-caminho de $T$ se $\sigma_{P}^{m} \leq \delta \frac{\mathbb{S}}{6}$ e $\rho_{P}^{m} \leq \delta \frac{\mathbb{P}}{6}$.

Definição 5.7. Dados $0<\delta \leq \frac{1}{2}$ e uma árvore $T$ geradora de $G$, um conjunto de $\delta$ - $\sigma \rho$-caminhos de $T$ internamente disjuntos dois a dois $Y=\left\{P_{1}, P_{2}, \ldots, P_{l}\right\}$ é um $\delta$ - $\sigma \rho$-spine se $S=\bigcup_{i=1}^{l} P_{i}$ é um $\delta$ - $\sigma \rho$-separador minimal de $T$. Denota-se por ext $(Y)$ o conjunto de vértices que são extremos dos caminhos em $Y$.

Finalmente introduzimos a definição de $k$-centro $\delta$ - $\sigma \rho$-balanceado.

Definição 5.8. Um k-centro $X$ de $G$ é $\delta$ - $\sigma \rho$-balanceado ou simplesmente $\delta$-balanceado, se tem um esqueleto $\tau$, tal que toda folha u de $\tau$ satisfaz que $\sigma\left(V\left(X, E_{\tau}, u\right)\right)>\delta \mathbb{S}$ ou $\rho\left(V\left(X, E_{\tau}, u\right)\right)>\delta \mathbb{P}$. Neste caso dizemos que $X$ é $\delta$-balanceado sobre $\tau$.

\subsection{Lema de aproximação dado um spine}

Usando as definições da seção anterior, vamos provar que para quaisquer $0<\delta \leq \frac{1}{2}$, árvore geradora $T$ de $G$ e $\delta$ - $\sigma \rho$-spine $Y$ de $T$, existe um $|\operatorname{ext}(Y)|$-centro cujo custo de comunicação é limitado por $\frac{1}{1-\delta} C(T)$. Na Seção 5.6 demonstramos a existência de um $\delta$ - $\sigma \rho$-spine para quaisquer

\footnotetext{
${ }^{1}$ Usamos a mesma figura apresentada no capítulo anterior para o $p$-OCT, porém mais detalhada e adaptada ao problema que tratamos neste capítulo.
} 
$0<\delta \leq \frac{1}{2}$ e árvore geradora $T$ de $G$. A partir destes resultados, temos o teorema apresentado na Seção 5.7.

Lema 5.9. Dados $0<\delta \leq \frac{1}{2}$, uma árvore geradora $T$ de $G$ e um $\delta$ - $\sigma \rho$-spine $Y$ de $T$, existe um $|\operatorname{ext}(Y)|$-centro $\delta$-balanceado $X$ de $G$ que satisfaz $C(X) \leq \frac{1}{1-\delta} C(T)$.

Para provar este lema usamos as três proposições a seguir, que demonstraremos nas seções seguintes. A proposição 5.10 estabelece uma propriedade referente ao custo de comunicação em $\delta$ - $\sigma \rho$-caminhos, enquanto que as proposições 5.11 e 5.12 oferecem uma limitante superior em função do custo de comunicação de uma árvore geradora $T$ de $G$, para o custo de comunicação de um $k$-centro obtido a partir de um $\delta$ - $\sigma \rho$-spine de $T$.

Proposição 5.10. Dados $0<\delta \leq \frac{1}{2}$ e um $\delta$ - $\sigma \rho$-caminho $P$ de um $\delta$ - $\sigma \rho$-spine de alguma árvore geradora $T$ de $G$, temos:

$$
\begin{aligned}
& \left(\mathbb{S}+\mathbb{P}-\sigma_{P}^{m}-\rho_{P}^{m}\right)\left(\sigma_{P}^{f} \rho_{P}^{l}+\sigma_{P}^{l} \rho_{P}^{f}\right) \omega(P) \\
+ & \left(\sigma_{P}^{l}+\rho_{P}^{l}\right)\left(\omega(P)\left(\sigma_{P}^{m} \rho_{P}^{f}+\sigma_{P}^{f} \rho_{P}^{m}\right)+\mathbb{S P}^{l}(P)+\mathbb{P S}^{l}(P)\right) \\
+ & \left(\sigma_{P}^{f}+\rho_{P}^{f}\right)\left(\omega(P)\left(\sigma_{P}^{m} \rho_{P}^{l}+\sigma_{P}^{l} \rho_{P}^{m}\right)+\mathbb{S P}^{f}(P)+\mathbb{P S}^{f}(P)\right) \\
\leq & \frac{6+5 \delta}{6}(\mathbb{S}+\mathbb{P})\left(\sigma_{P}^{f} \rho_{P}^{l}+\sigma_{P}^{l} \rho_{P}^{f}+\sigma_{P}^{\min } \rho_{P}^{m}+\sigma_{P}^{m} \rho_{P}^{\min }\right) \omega(P) \\
& +(\mathbb{S}+\mathbb{P})\left(\left(\sigma_{P}^{\max }-\sigma_{P}^{\min }\right) \mathbb{P}(P)+\left(\rho_{P}^{\max }-\rho_{P}^{\min }\right) \mathbb{S}(P)\right) .
\end{aligned}
$$

Proposição 5.11. Dados $0<\delta \leq \frac{1}{2}$, uma árvore geradora $T$ de $G$ e um $\delta$ - $\sigma \rho$-spine $Y$ de $T$, existe um $|\operatorname{ext}(Y)|$-centro $\delta$-balanceado $X$ de $G$ que satisfaz:

$$
\begin{aligned}
C(X) \leq & \sum_{P \in Y}\left(\sigma_{P}^{f} \rho_{P}^{l}+\rho_{P}^{f} \sigma_{P}^{l}\right) \omega(P)+\min \left\{\Delta_{f l}(P), \Delta_{l f}(P)\right\} \\
& +\mathbb{P} \sum_{u \in V_{G}} \sigma(u) d(T, u, S)+\mathbb{S} \sum_{u \in V_{G}} \rho(u) d(T, u, S) .
\end{aligned}
$$

Onde $\Delta_{w z}(P)=\omega(P)\left(\sigma_{P}^{m} \rho_{P}^{w}+\rho_{P}^{m} \sigma_{P}^{w}\right)+\mathbb{P S}^{z}(P)+\mathbb{S P}^{z}(P), w, z \in\{f, l\}$, e $S=\bigcup_{P \in Y} P$.

Observe que esta proposição garante a existência de um $|\operatorname{ext}(Y)|$-centro $X$ de $G$ construído a partir de um $\delta$ - $\sigma \rho$-spine $Y$ de uma árvore geradora de $G$ e ainda dá uma limitante superior para o custo de comunicação de $X$. A próxima proposição dá uma limitante inferior para o custo de comunicação de uma árvore geradora de $G$.

Proposição 5.12. Dados $0<\delta \leq \frac{1}{2}$, uma árvore geradora $T$ de $G$ e um $\delta$ - $\sigma \rho$-spine $Y$ de $T$, temos:

$$
\begin{aligned}
C(T) \geq & \sum_{P \in Y}\left(\rho_{P}^{l} \sigma_{P}^{f}+\rho_{P}^{f} \sigma_{P}^{l}+\rho_{P}^{\min } \sigma_{P}^{m}+\sigma_{P}^{\min } \rho_{P}^{m}\right) \omega(P) \\
& +\sum_{P \in Y}\left(\sigma_{P}^{\max }-\sigma_{P}^{\min }\right) \mathbb{P}(P)+\left(\rho_{P}^{\max }-\rho_{P}^{\min }\right) \mathbb{S}(P) \\
& +(1-\delta)\left(\mathbb{S} \sum_{u \in V_{G}} \rho(u) d(T, u, S)+\mathbb{P} \sum_{u \in V_{G}} \sigma(u) d(T, u, S)\right) .
\end{aligned}
$$

Onde $S=\bigcup_{P \in Y} P$. 
Usando estas proposições vamos demonstrar o lema 5.9, que garante a existência de um centro com esqueleto no conjunto $\operatorname{ext}(Y)$ de um dado $\delta$ - $\sigma \rho$-spine $Y$ de uma árvore geradora $T$, tal que o custo de comunicação dele é limitado superiormente por $\frac{1}{1-\delta} C(T)$. Segue a prova do lema 5.9.

Demonstração. Sejam $S=\bigcup_{P \in Y} P$ o $\delta$ - $\sigma \rho$-separador minimal associado a $Y$ e $X$ o $|\operatorname{ext}(Y)|$-centro $\delta$-balanceado de $G$ dado pela proposição 5.11, então:

$$
\begin{aligned}
C(X) \leq & \sum_{P \in Y}\left(\sigma_{P}^{f} \rho_{P}^{l}+\rho_{P}^{f} \sigma_{P}^{l}\right) \omega(P)+\min \left\{\Delta_{f l}(P), \Delta_{l f}(P)\right\} \\
& +\mathbb{P} \sum_{u \in V_{G}} \sigma(u) d(T, u, S)+\mathbb{S} \sum_{u \in V_{G}} \rho(u) d(T, u, S) \\
\leq & \sum_{P \in Y} \frac{\mathbb{S}+\mathbb{P}-\rho_{P}^{\boldsymbol{m}}-\boldsymbol{\sigma}_{\boldsymbol{P}}^{\boldsymbol{m}}}{\mathbb{S}+\mathbb{P}-\boldsymbol{\rho}_{\boldsymbol{P}}^{\boldsymbol{m}}-\boldsymbol{\sigma}_{\boldsymbol{P}}^{\boldsymbol{m}}}\left(\sigma_{P}^{f} \rho_{P}^{l}+\rho_{P}^{f} \sigma_{P}^{l}\right) \omega(P)+\min \left\{\Delta_{f l}(P), \Delta_{l f}(P)\right\} \\
& +\mathbb{P} \sum_{u \in V_{G}} \sigma(u) d(T, u, S)+\mathbb{S} \sum_{u \in V_{G}} \rho(u) d(T, u, S) .
\end{aligned}
$$

Como o mínimo entre dois números é menor ou igual que a média com pesos entre eles, então, para cada $P \in Y$ temos:

$$
\begin{aligned}
& \min \left\{\Delta_{f l}(P), \Delta_{l f}(P)\right\} \\
\leq & \sum_{P \in Y} \frac{\sigma_{P}^{l}+\rho_{P}^{l}}{\sigma_{P}^{l}+\rho_{P}^{l}+\sigma_{P}^{f}+\rho_{P}^{f}} \Delta_{f l}(P)+\frac{\sigma_{P}^{f}+\rho_{P}^{f}}{\sigma_{P}^{l}+\rho_{P}^{l}+\sigma_{P}^{f}+\rho_{P}^{f}} \Delta_{l f}(P) .
\end{aligned}
$$

Logo,

$$
\begin{aligned}
C(X) \leq & \sum_{P \in Y} \frac{\mathbb{S}+\mathbb{P}-\rho_{P}^{m}-\sigma_{P}^{m}}{\mathbb{S}+\mathbb{P}-\rho_{P}^{m}-\sigma_{P}^{m}}\left(\sigma_{P}^{f} \rho_{P}^{l}+\rho_{P}^{f} \sigma_{P}^{l}\right) \omega(P) \\
& +\sum_{\boldsymbol{P} \in \boldsymbol{Y}} \frac{\boldsymbol{\sigma}_{\boldsymbol{P}}^{l}+\boldsymbol{\rho}_{\boldsymbol{P}}^{\boldsymbol{l}}}{\boldsymbol{\sigma}_{\boldsymbol{P}}^{l}+\boldsymbol{\rho}_{\boldsymbol{P}}^{\boldsymbol{l}}+\boldsymbol{\sigma}_{\boldsymbol{P}}^{f}+\boldsymbol{\rho}_{\boldsymbol{P}}^{f}} \boldsymbol{\Delta}_{f l}(\boldsymbol{P})+\frac{\boldsymbol{\sigma}_{\boldsymbol{P}}^{f}+\boldsymbol{\rho}_{\boldsymbol{P}}^{f}}{\boldsymbol{\sigma}_{\boldsymbol{P}}^{l}+\boldsymbol{\rho}_{\boldsymbol{P}}^{l}+\boldsymbol{\sigma}_{\boldsymbol{P}}^{f}+\boldsymbol{\rho}_{\boldsymbol{P}}^{f}} \boldsymbol{\Delta}_{l f}(\boldsymbol{P}) \\
& +\mathbb{P} \sum_{u \in V_{G}} \sigma(u) d(T, u, S)+\mathbb{S} \sum_{u \in V_{G}} \rho(u) d(T, u, S) .
\end{aligned}
$$

Todo caminho $P$ satisfaz $\mathbb{S}+\mathbb{P}-\boldsymbol{\sigma}_{\boldsymbol{P}}^{\boldsymbol{m}}-\boldsymbol{\rho}_{\boldsymbol{P}}^{\boldsymbol{m}}=\sigma_{P}^{f}+\sigma_{P}^{l}+\rho_{P}^{f}+\rho_{P}^{l}$, logo:

$$
\begin{aligned}
C(X) \leq & \sum_{P \in Y} \frac{\mathbb{S}+\mathbb{P}-\rho_{P}^{m}-\sigma_{P}^{m}}{\mathbb{S}+\mathbb{P}-\rho_{P}^{m}-\sigma_{P}^{m}}\left(\sigma_{P}^{f} \rho_{P}^{l}+\rho_{P}^{f} \sigma_{P}^{l}\right) \omega(P) \\
& +\sum_{P \in Y} \frac{\sigma_{P}^{l}+\rho_{P}^{l}}{\mathbb{S}+\mathbb{P}-\boldsymbol{\sigma}_{\boldsymbol{P}}^{m}-\boldsymbol{\sigma}_{\boldsymbol{P}}^{m}} \Delta_{f l}(P)+\frac{\sigma_{P}^{f}+\rho_{P}^{f}}{\mathbb{S}+\mathbb{P}-\boldsymbol{\sigma}_{\boldsymbol{P}}^{m}-\boldsymbol{\sigma}_{\boldsymbol{P}}^{m}} \Delta_{l f}(P) \\
& +\mathbb{P} \sum_{u \in V_{G}} \sigma(u) d(T, u, S)+\mathbb{S} \sum_{u \in V_{G}} \rho(u) d(T, u, S) .
\end{aligned}
$$

Como cada $P \in Y$ é um $\delta$ - $\sigma \rho$-caminho, então, aplicando a proposição 5.10 temos:

$$
\sum_{P \in Y} \frac{\mathbb{S}+\mathbb{P}-\rho_{P}^{m}-\sigma_{P}^{m}}{\mathbb{S}+\mathbb{P}-\rho_{P}^{m}-\sigma_{P}^{m}}\left(\sigma_{P}^{f} \rho_{P}^{l}+\rho_{P}^{f} \sigma_{P}^{l}\right) \omega(P)
$$




$$
\begin{aligned}
& +\sum_{P \in Y} \frac{\sigma_{P}^{l}+\rho_{P}^{l}}{\mathbb{S}+\mathbb{P}-\sigma_{P}^{m}-\sigma_{P}^{m}} \Delta_{f l}(P)+\frac{\sigma_{P}^{f}+\rho_{P}^{f}}{\mathbb{S}+\mathbb{P}-\sigma_{P}^{m}-\sigma_{P}^{m}} \Delta_{l f}(P) \\
\leq & \sum_{P \in Y} \frac{\left(\frac{6+5 \delta}{6}\right)(\mathbb{S}+\mathbb{P})}{\mathbb{S}+\mathbb{P}-\rho_{P}^{m}-\sigma_{P}^{m}}\left(\sigma_{P}^{f} \rho_{P}^{l}+\rho_{P}^{f} \sigma_{P}^{l}+\sigma_{P}^{\min } \rho_{P}^{m}+\rho_{P}^{\min } \sigma_{P}^{m}\right) \omega(P) \\
& +\sum_{P \in Y} \frac{\mathbb{S}+\mathbb{P}}{\mathbb{S}+\mathbb{P}-\rho_{P}^{m}-\sigma_{P}^{m}}\left(\left(\sigma_{P}^{\max }-\sigma_{P}^{\min }\right) \mathbb{P}(P)+\left(\rho_{P}^{\max }-\rho_{P}^{\min }\right) \mathbb{S}(P)\right) .
\end{aligned}
$$

Logo:

$$
\begin{aligned}
& C(X) \leq \sum_{P \in Y} \frac{\left(\frac{6+5 \delta}{6}\right)(\mathbb{S}+\mathbb{P})}{\mathbb{S}+\mathbb{P}-\rho_{P}^{m}-\sigma_{P}^{m}}\left(\sigma_{P}^{f} \rho_{P}^{l}+\rho_{P}^{f} \sigma_{P}^{l}+\sigma_{P}^{\min } \rho_{P}^{m}+\rho_{P}^{\min } \sigma_{P}^{m}\right) \omega(P) \\
& +\sum_{P \in Y} \frac{\mathbb{S}+\mathbb{P}}{\mathbb{S}+\mathbb{P}-\rho_{P}^{m}-\sigma_{P}^{m}}\left(\left(\sigma_{P}^{\max }-\sigma_{P}^{\min }\right) \mathbb{P}(P)+\left(\rho_{P}^{\max }-\rho_{P}^{\min }\right) \mathbb{S}(P)\right) \\
& +\mathbb{P} \sum_{u \in V_{G}} \sigma(u) d(T, u, S)+\mathbb{S} \sum_{u \in V_{G}} \rho(u) d(T, u, S) .
\end{aligned}
$$

Observe que todo $\delta$ - $\sigma \rho$-caminho satisfaz: $\rho_{P}^{m}+\sigma_{P}^{m} \leq \frac{\delta}{6} \mathbb{S}+\frac{\delta}{6} \mathbb{P}=\frac{\delta}{6}(\mathbb{S}+\mathbb{P})$, logo:

$$
\begin{aligned}
C(X) \leq & \sum_{P \in Y} \frac{\left(\frac{6+5 \delta}{6}\right)(\mathbb{S}+\mathbb{P})}{\mathbb{S}+\frac{\boldsymbol{\delta}}{\mathbf{6}}(\mathbb{S}+\mathbb{P})}\left(\sigma_{P}^{f} \rho_{P}^{l}+\rho_{P}^{f} \sigma_{P}^{l}+\sigma_{P}^{\min } \rho_{P}^{m}+\rho_{P}^{\min } \sigma_{P}^{m}\right) \omega(P) \\
& +\sum_{P \in Y} \frac{\mathbb{S}+\mathbb{P}}{\mathbb{S}+\mathbb{P}-\frac{\boldsymbol{\delta}}{\mathbf{6}}(\mathbb{S}+\mathbb{P})}\left(\left(\sigma_{P}^{\max }-\sigma_{P}^{\min }\right) \mathbb{P}(P)+\left(\rho_{P}^{\max }-\rho_{P}^{\min }\right) \mathbb{S}(P)\right) \\
& +\mathbb{P} \sum_{u \in V_{G}} \sigma(u) d(T, u, S)+\mathbb{S} \sum_{u \in V_{G}} \rho(u) d(T, u, S) \\
= & \frac{\mathbf{6}+\mathbf{5} \boldsymbol{\delta}}{\mathbf{6}-\boldsymbol{\delta}} \sum_{P \in Y}\left(\sigma_{P}^{f} \rho_{P}^{l}+\rho_{P}^{f} \sigma_{P}^{l}+\sigma_{P}^{\min } \rho_{P}^{m}+\rho_{P}^{\min } \sigma_{P}^{m}\right) \omega(P) \\
& +\frac{\mathbf{6}}{\mathbf{6}-\boldsymbol{\delta}} \sum_{P \in Y}\left(\left(\sigma_{P}^{\max }-\sigma_{P}^{\min }\right) \mathbb{P}(P)+\left(\rho_{P}^{\max }-\rho_{P}^{\min }\right) \mathbb{S}(P)\right) \\
& +\frac{\mathbf{1}}{\mathbf{1}-\boldsymbol{\delta}}(\mathbf{1}-\boldsymbol{\delta})\left(\mathbb{P} \sum_{u \in V_{G}} \sigma(u) d(T, u, S)+\mathbb{S} \sum_{u \in V_{G}} \rho(u) d(T, u, S)\right) .
\end{aligned}
$$

Como $\frac{\mathbf{6}+\mathbf{5} \boldsymbol{\delta}}{\mathbf{6 - \delta}}=\frac{(6+5 \delta)(1-\delta)}{(6-\delta)(1-\delta)}=\frac{6-\delta-5 \delta^{2}}{(6-\delta)(1-\delta)}<\frac{6-\delta}{(6-\delta)(1-\delta)}=\frac{\mathbf{1}}{\mathbf{1 - \delta}}$ e $\frac{\mathbf{6}}{\mathbf{6 - \delta}}<\frac{6+5 \delta}{6-\delta}<\frac{\mathbf{1}}{\mathbf{1 - \boldsymbol { \delta }}}$, aplicando a proposição 5.12 concluímos:

$$
C(X) \leq \frac{1}{1-\delta} C(T)
$$

\subsection{Propriedade de alguns caminhos}

Esta seção mostra o resultado dado pela proposição 5.10. Para isto será necessário provar antes o seguinte fato, que dado um caminho de uma árvore geradora de $G$, este limita inferior e superiormente o requerimento que atravessa o caminho. 
Fato 5.13. Dada uma árvore geradora $T$ de $G$ e um caminho $P$ de $T$ :

$$
\left(\sigma_{P}^{\min } \rho_{P}^{\max }+\sigma_{P}^{\max } \rho_{P}^{\min }\right) \leq\left(\sigma_{P}^{f} \rho_{P}^{l}+\sigma_{P}^{l} \rho_{P}^{f}\right) \leq\left(\sigma_{P}^{\max } \rho_{P}^{\max }+\sigma_{P}^{\min } \rho_{P}^{\min }\right)
$$

Demonstração. Por definição $\sigma_{P}^{\min } \leq \sigma_{P}^{\max }, \operatorname{logo} \sigma_{P}^{\max }=\sigma_{P}^{\min }+\xi$ com $\xi \geq 0$, o que implica:

$$
\sigma_{P}^{\min } \rho_{P}^{\max }+\sigma_{P}^{\max } \rho_{P}^{\min }=\sigma_{P}^{\max } \rho_{P}^{\max }+\sigma_{P}^{\min } \rho_{P}^{\min }-\xi\left(\rho_{P}^{\max }-\rho_{P}^{\min }\right) .
$$

Também por definição $\rho_{P}^{\min } \leq \rho_{P}^{\max }, \operatorname{logo}:$

$$
\sigma_{P}^{\min } \rho_{P}^{\max }+\sigma_{P}^{\max } \rho_{P}^{\min } \leq \sigma_{P}^{\max } \rho_{P}^{\max }+\sigma_{P}^{\min } \rho_{P}^{\min } .
$$

Como

$$
\sigma_{P}^{f} \rho_{P}^{l}+\sigma_{P}^{l} \rho_{P}^{f} \in\left\{\sigma_{P}^{\min } \rho_{P}^{\max }+\sigma_{P}^{\max } \rho_{P}^{\min }, \sigma_{P}^{\max } \rho_{P}^{\max }+\sigma_{P}^{\min } \rho_{P}^{\min }\right\}
$$

é possível concluir:

$$
\left(\sigma_{P}^{\min } \rho_{P}^{\max }+\sigma_{P}^{\max } \rho_{P}^{\min }\right) \leq\left(\sigma_{P}^{f} \rho_{P}^{l}+\sigma_{P}^{l} \rho_{P}^{f}\right) \leq\left(\sigma_{P}^{\max } \rho_{P}^{\max }+\sigma_{P}^{\min } \rho_{P}^{\min }\right)
$$

Agora passamos para a demonstração da proposição 5.10 que para $0<\delta \leq \frac{1}{2}$, dado um $\delta$ - $\sigma \rho$ caminho $P$ de um $\delta$ - $\sigma \rho$-spine de alguma árvore $T$ geradora de $G$, garante:

$$
\begin{aligned}
& \left(\mathbb{S}+\mathbb{P}-\sigma_{P}^{m}-\rho_{P}^{m}\right)\left(\sigma_{P}^{f} \rho_{P}^{l}+\sigma_{P}^{l} \rho_{P}^{f}\right) \omega(P) \\
+ & \left(\sigma_{P}^{l}+\rho_{P}^{l}\right)\left(\omega(P)\left(\sigma_{P}^{m} \rho_{P}^{f}+\sigma_{P}^{f} \rho_{P}^{m}\right)+\mathbb{S P}^{l}(P)+\mathbb{P S}^{l}(P)\right) \\
+ & \left(\sigma_{P}^{f}+\rho_{P}^{f}\right)\left(\omega(P)\left(\sigma_{P}^{m} \rho_{P}^{l}+\sigma_{P}^{l} \rho_{P}^{m}\right)+\mathbb{S P}^{f}(P)+\mathbb{P S}^{f}(P)\right) \\
\leq & \frac{6+5 \delta}{6}(\mathbb{S}+\mathbb{P})\left(\sigma_{P}^{f} \rho_{P}^{l}+\sigma_{P}^{l} \rho_{P}^{f}+\sigma_{P}^{\min } \rho_{P}^{m}+\sigma_{P}^{m} \rho_{P}^{\min }\right) \omega(P) \\
+ & (\mathbb{S}+\mathbb{P})\left(\left(\sigma_{P}^{\max }-\sigma_{P}^{\min }\right) \mathbb{P}(P)+\left(\rho_{P}^{\max }-\rho_{P}^{\min }\right) \mathbb{S}(P)\right) .
\end{aligned}
$$

Demonstração. Considere $S=\cup_{Q \in Y} Q$ o separador minimal de $T$ obtido a partir de $Y$, então denote por $\phi(P)$ :

$$
\begin{aligned}
\phi(P)= & \left(\mathbb{S}+\mathbb{P}-\sigma_{P}^{m}-\rho_{P}^{m}\right)\left(\sigma_{P}^{f} \rho_{P}^{l}+\sigma_{P}^{l} \rho_{P}^{f}\right) \omega(P) \\
& +\left(\sigma_{P}^{l}+\rho_{P}^{l}\right)\left(\omega(P)\left(\sigma_{P}^{m} \rho_{P}^{f}+\sigma_{P}^{f} \rho_{P}^{m}\right)+\mathbb{S P}^{l}(P)+\mathbb{P S}^{l}(P)\right) \\
& +\left(\sigma_{P}^{f}+\rho_{P}^{f}\right)\left(\omega(P)\left(\sigma_{P}^{m} \rho_{P}^{l}+\sigma_{P}^{l} \rho_{P}^{m}\right)+\mathbb{S P}^{f}(P)+\mathbb{P S}^{f}(P)\right) .
\end{aligned}
$$

Observe que na equação acima, a primeira linha é igual à soma das linhas (5.1) e (5.2), a segunda linha é igual à soma das linhas (5.3), (5.4) e (5.5), e a terceira linha é igual à soma das linhas (5.6), (5.7) e (5.8).

$$
\begin{aligned}
\phi(P)= & (\mathbb{S}+\mathbb{P})\left(\sigma_{P}^{f} \rho_{P}^{l}+\sigma_{P}^{l} \rho_{P}^{f}\right) \omega(P) \\
& +\left(-\sigma_{P}^{m}-\rho_{P}^{m}\right)\left(\sigma_{P}^{f} \rho_{P}^{l}+\sigma_{P}^{l} \rho_{P}^{f}\right) \omega(P)
\end{aligned}
$$




$$
\begin{aligned}
& +\left(\sigma_{P}^{m} \sigma_{P}^{l} \rho_{P}^{f}+\rho_{P}^{m} \sigma_{P}^{f} \rho_{P}^{l}\right) \omega(P) \\
& +\left(\sigma_{P}^{m} \rho_{P}^{f} \rho_{P}^{l}+\rho_{P}^{m} \sigma_{P}^{f} \sigma_{P}^{l}\right) \omega(P) \\
& +\mathbb{S} \sigma_{P}^{l} \mathbb{P}^{l}(P)+\mathbb{P} \sigma_{P}^{l} \mathbb{S}^{l}(P)+\mathbb{S} \rho_{P}^{l} \mathbb{P}^{l}(P)+\mathbb{P} \rho_{P}^{l} \mathbb{S}^{l}(P) \\
& +\left(\sigma_{P}^{m} \sigma_{P}^{f} \rho_{P}^{l}+\rho_{P}^{m} \sigma_{P}^{l} \rho_{P}^{f}\right) \omega(P) \\
& +\left(\sigma_{P}^{m} \rho_{P}^{f} \rho_{P}^{l}+\rho_{P}^{m} \sigma_{P}^{f} \sigma_{P}^{l}\right) \omega(P) \\
& +\mathbb{S} \sigma_{P}^{f} \mathbb{P}^{f}(P)+\mathbb{P} \sigma_{P}^{f} \mathbb{S}^{f}(P)+\mathbb{S} \rho_{P}^{f} \mathbb{P}^{f}(P)+\mathbb{P} \rho_{P}^{f} \mathbb{S}^{f}(P) .
\end{aligned}
$$

Note que $(5.2)+(5.3)+(5.6)$ é igual a zero, e que as linhas (5.4) e (5.7) são iguais, logo:

$$
\begin{aligned}
\phi(P)= & (\mathbb{S}+\mathbb{P})\left(\sigma_{P}^{f} \rho_{P}^{l}+\sigma_{P}^{l} \rho_{P}^{f}\right) \omega(P) \\
& +2\left(\sigma_{P}^{m} \rho_{P}^{f} \rho_{P}^{l}+\rho_{P}^{m} \sigma_{P}^{f} \sigma_{P}^{l}\right) \omega(P) \\
& +\mathbb{S} \sigma_{P}^{l} \mathbb{P}^{l}(P)+\mathbb{P} \sigma_{P}^{l} \mathbb{S}^{l}(P)+\mathbb{S} \rho_{P}^{l} \mathbb{P}^{l}(P)+\mathbb{P} \rho_{P}^{l} \mathbb{S}^{l}(P) \\
& +\mathbb{S} \sigma_{P}^{f} \mathbb{P}^{f}(P)+\mathbb{P} \sigma_{P}^{f} \mathbb{S}^{f}(P)+\mathbb{S} \rho_{P}^{f} \mathbb{P}^{f}(P)+\mathbb{P} \rho_{P}^{f} \mathbb{S}^{f}(P) .
\end{aligned}
$$

Como:

$$
\begin{aligned}
\left\{\sigma_{P}^{\max }, \sigma_{P}^{\min }\right\} & =\left\{\sigma_{P}^{f}, \sigma_{P}^{l}\right\}, \\
\left\{\rho_{P}^{\max }, \rho_{P}^{\min }\right\} & =\left\{\rho_{P}^{f}, \rho_{P}^{l}\right\}, \\
\sigma_{P}^{m} \omega(P) & =\mathbb{S}^{f}(P)+\mathbb{S}^{l}(P), \mathrm{e} \\
\rho_{P}^{m} \omega(P) & =\mathbb{P}^{f}(P)+\mathbb{P}^{l}(P) .
\end{aligned}
$$

Então, as linhas (5.9) e (5.12) são iguais, e $(5.10)+(5.11)=(5.13)+(5.14)+(5.15)=(5.17)+$ $(5.18)+(5.19)$.

$$
\begin{aligned}
\phi(P)= & (\mathbb{S}+\mathbb{P})\left(\sigma_{P}^{f} \rho_{P}^{l}+\sigma_{P}^{l} \rho_{P}^{f}\right) \omega(P) \\
& +2\left(\sigma_{P}^{m} \rho_{P}^{\max } \rho_{P}^{\min }+\rho_{P}^{m} \sigma_{P}^{\max } \sigma_{P}^{\min }\right) \omega(P) \\
& +\mathbb{S} \sigma_{P}^{\max } \mathbb{P}(P)+\mathbb{P} \sigma_{P}^{\max } \mathbb{S}(P)+\mathbb{S} \rho_{P}^{\max } \mathbb{P}(P)+\mathbb{P} \rho_{P}^{\max } \mathbb{S}(P) \\
& +\mathbb{P} \sigma_{P}^{\min }\left(\sigma_{P}^{m} \omega(P)-\mathbb{S}(P)\right)+\mathbb{P} \sigma_{P}^{\min }\left(\rho_{P}^{m} \omega(P)-\mathbb{S}(P)\right) \\
& +\mathbb{S} \rho_{P}^{\min }\left(\sigma_{P}^{m} \omega(P)-\mathbb{P}(P)\right)+\mathbb{S} \rho_{P}^{\min }\left(\rho_{P}^{m} \omega(P)-\mathbb{P}(P)\right) \\
= & (\mathbb{S}+\mathbb{P})\left(\sigma_{P}^{f} \rho_{P}^{l}+\sigma_{P}^{l} \rho_{P}^{f}\right) \omega(P) \\
& +\mathbf{2}\left(\boldsymbol{\sigma}_{\boldsymbol{P}}^{m} \boldsymbol{\rho}_{\boldsymbol{P}}^{\max } \boldsymbol{\rho}_{\boldsymbol{P}}^{\min }+\boldsymbol{\rho}_{\boldsymbol{P}}^{\boldsymbol{m}} \boldsymbol{\sigma}_{\boldsymbol{P}}^{\max } \boldsymbol{\sigma}_{\boldsymbol{P}}^{\min }\right) \boldsymbol{\omega}(\boldsymbol{P}) \\
& +\mathbb{S}\left(\sigma_{P}^{\max }-\sigma_{P}^{\min }\right) \mathbb{P}(P)+\mathbb{P}\left(\rho_{P}^{\max }-\rho_{P}^{\min }\right) \mathbb{S}(P) \\
& +\mathbb{P}\left(\sigma_{P}^{\max }-\sigma_{P}^{\min }\right) \mathbb{S}(P)+\mathbb{S}\left(\rho_{P}^{\max }-\rho_{P}^{\min }\right) \mathbb{P}(P) \\
& +\left(\mathbb{P} \boldsymbol{\sigma}_{\boldsymbol{P}}^{\min } \boldsymbol{\sigma}_{\boldsymbol{P}}^{m}+\mathbb{S} \sigma_{P}^{\min } \rho_{P}^{m}+\mathbb{P} \rho_{P}^{\min } \sigma_{P}^{m}+\mathbb{S} \boldsymbol{\rho}_{\boldsymbol{P}}^{\min } \boldsymbol{\rho}_{\boldsymbol{P}}^{\boldsymbol{m}}\right) \omega(P) .
\end{aligned}
$$

Agrupando o primeiro e último termos da linha (5.19) com a (5.16) temos: 


$$
\begin{aligned}
\phi(P)= & (\mathbb{S}+\mathbb{P})\left(\sigma_{P}^{f} \rho_{P}^{l}+\sigma_{P}^{l} \rho_{P}^{f}\right) \omega(P) \\
& +\left(2 \sigma_{P}^{m} \rho_{P}^{\max } \rho_{P}^{\min }+2 \rho_{P}^{m} \sigma_{P}^{\max } \sigma_{P}^{\min }+\mathbb{P} \sigma_{P}^{\min } \sigma_{P}^{m}+\mathbb{S} \rho_{P}^{\min } \rho_{P}^{m}\right) \omega(P) \\
& +\mathbb{S}\left(\sigma_{P}^{\max }-\sigma_{P}^{\max }\right) \mathbb{P}(P)+\mathbb{P}\left(\rho_{P}^{\max }-\rho_{P}^{\min }\right) \mathbb{S}(P) \\
& +\mathbb{P}\left(\sigma_{P}^{\max }-\sigma_{P}^{\min }\right) \mathbb{S}(P)+\mathbb{S}\left(\rho_{P}^{\max }-\rho_{P}^{\min }\right) \mathbb{P}(P) \\
& +\mathbb{S} \sigma_{P}^{\min } \rho_{P}^{m} \omega(P)+\mathbb{P} \rho_{P}^{\min } \sigma_{P}^{m} \omega(P) .
\end{aligned}
$$

Adicionando $\mathbb{P} \sigma_{P}^{\min } \rho_{P}^{m} \omega(P)+\mathbb{S} \rho_{P}^{\min } \sigma_{P}^{m} \omega(P)-\left(\mathbb{P} \sigma_{P}^{\min } \rho_{P}^{m} \omega(P)+\mathbb{S} \rho_{P}^{\min } \sigma_{P}^{m} \omega(P)\right)=0$ à linha (5.20) obtemos:

$$
\begin{aligned}
\phi(P)= & (\mathbb{S}+\mathbb{P})\left(\boldsymbol{\sigma}_{\boldsymbol{P}}^{\boldsymbol{f}} \boldsymbol{\rho}_{\boldsymbol{P}}^{\boldsymbol{l}}+\boldsymbol{\sigma}_{\boldsymbol{P}}^{\boldsymbol{l}} \boldsymbol{\rho}_{\boldsymbol{P}}^{\boldsymbol{f}}\right) \boldsymbol{\omega}(\boldsymbol{P}) \\
& +\left(2 \sigma_{P}^{m} \rho_{P}^{\max } \rho_{P}^{\min }+2 \rho_{P}^{m} \sigma_{P}^{\max } \sigma_{P}^{\min }+\mathbb{P} \sigma_{P}^{\min } \sigma_{P}^{m}+\mathbb{S} \rho_{P}^{\min } \rho_{P}^{m}\right) \omega(P) \\
& +\mathbb{S}\left(\sigma_{P}^{\max }-\sigma_{P}^{\max }\right) \mathbb{P}(P)+\mathbb{P}\left(\rho_{P}^{\max }-\rho_{P}^{\min }\right) \mathbb{S}(P) \\
& +\mathbb{P}\left(\sigma_{P}^{\max }-\sigma_{P}^{\min }\right) \mathbb{S}(P)+\mathbb{S}\left(\rho_{P}^{\max }-\rho_{P}^{\min }\right) \mathbb{P}(P) \\
& +(\mathbb{S}+\mathbb{P})\left(\boldsymbol{\sigma}_{\boldsymbol{P}}^{\min } \boldsymbol{\rho}_{\boldsymbol{P}}^{\boldsymbol{m}}+\boldsymbol{\rho}_{\boldsymbol{P}}^{\min } \boldsymbol{\sigma}_{\boldsymbol{P}}^{\boldsymbol{m}}\right) \boldsymbol{\omega}(\boldsymbol{P})-\left(\mathbb{P} \sigma_{P}^{\min } \rho_{P}^{m}+\mathbb{S} \rho_{P}^{\min } \sigma_{P}^{m}\right) \omega(P)
\end{aligned}
$$

Agrupando a primeira linha da equação acima com os termos não negativos da última obtemos:

$$
\begin{aligned}
\phi(P)= & (\mathbb{S}+\mathbb{P})\left(\boldsymbol{\sigma}_{\boldsymbol{P}}^{\boldsymbol{f}} \boldsymbol{\rho}_{\boldsymbol{P}}^{\boldsymbol{l}}+\boldsymbol{\sigma}_{\boldsymbol{P}}^{\boldsymbol{l}} \boldsymbol{\rho}_{\boldsymbol{P}}^{\boldsymbol{f}}+\boldsymbol{\sigma}_{\boldsymbol{P}}^{\min } \boldsymbol{\rho}_{\boldsymbol{P}}^{\boldsymbol{m}}+\boldsymbol{\rho}_{\boldsymbol{P}}^{\min } \boldsymbol{\sigma}_{\boldsymbol{P}}^{\boldsymbol{m}}\right) \boldsymbol{\omega}(\boldsymbol{P}) \\
& +\left(2 \sigma_{P}^{m} \rho_{P}^{\max } \rho_{P}^{\min }+2 \rho_{P}^{m} \sigma_{P}^{\max } \sigma_{P}^{\min }+\mathbb{P} \sigma_{P}^{\min } \sigma_{P}^{m}+\mathbb{S} \rho_{P}^{\min } \rho_{P}^{m}\right) \omega(P) \\
& +\mathbb{S}\left(\sigma_{P}^{\max }-\sigma_{P}^{\max }\right) \mathbb{P}(P)+\mathbb{P}\left(\rho_{P}^{\max }-\rho_{P}^{\min }\right) \mathbb{S}(P) \\
& +\mathbb{P}\left(\sigma_{P}^{\max }-\sigma_{P}^{\min }\right) \mathbb{S}(P)+\mathbb{S}\left(\rho_{P}^{\max }-\rho_{P}^{\min }\right) \mathbb{P}(P) \\
& -\left(\mathbb{P} \sigma_{P}^{\min } \rho_{P}^{m}+\mathbb{S} \rho_{P}^{\min } \sigma_{P}^{m}\right) \omega(P) .
\end{aligned}
$$

Observe que $\sigma_{P}^{m} \leq \frac{\delta}{6} \mathbb{S}, \rho_{P}^{m} \leq \frac{\delta}{6} \mathbb{P}, \mathbb{P} \geq \rho_{P}^{\max }$ e $\mathbb{S} \geq \sigma_{P}^{\max }$, logo:

$$
\begin{aligned}
& 2 \sigma_{P}^{m} \rho_{P}^{\max } \rho_{P}^{\min }+2 \rho_{P}^{m} \sigma_{P}^{\max } \sigma_{P}^{\min }+\mathbb{P} \sigma_{P}^{\min } \sigma_{P}^{m}+\mathbb{S} \rho_{P}^{\min } \rho_{P}^{m} \\
\leq \quad & 2 \frac{\boldsymbol{\delta}}{\mathbf{6}} \mathbb{S} \mathbb{P} \rho_{P}^{\min }+2 \frac{\boldsymbol{\delta}}{\mathbf{6}} \mathbb{P} S \sigma_{P}^{\min }+\mathbb{P} \sigma_{P}^{\min } \frac{\boldsymbol{\delta}}{\mathbf{6}} \mathbb{S}+\mathbb{S} \rho_{P}^{\min } \frac{\boldsymbol{\delta}}{\mathbf{6}} \mathbb{P} \\
= & \frac{\delta}{2}\left(\mathbb{S P} \rho_{P}^{\min }+\mathbb{S P} \sigma_{P}^{\min }\right) .
\end{aligned}
$$

Como $\rho_{P}^{\max } \geq \rho_{P}^{\min }, \sigma_{P}^{\max } \geq \sigma_{P}^{\min }, \mathbb{S}=\sigma_{P}^{\max }+\sigma_{P}^{\min }+\sigma_{P}^{m}$ e $\mathbb{P}=\rho_{P}^{\max }+\rho_{P}^{\min }+\rho_{P}^{m}$, temos:

$$
\begin{aligned}
& \mathbb{S P} \rho_{P}^{\min }+\mathbb{S P} \sigma_{P}^{\min } \\
\leq \quad & \mathbb{S P} \rho_{P}^{\min }+\mathbb{S P} \sigma_{P}^{\min } \\
& +\mathbb{S}\left(\rho_{P}^{\min }\left(\sigma_{P}^{\max }-\sigma_{P}^{\min }\right)+\rho_{P}^{\min } \sigma_{P}^{m}\right)+\mathbb{P}\left(\sigma_{P}^{\min }\left(\rho_{P}^{\max }-\rho_{P}^{\min }\right)+\sigma_{P}^{\min } \rho_{P}^{m}\right) .
\end{aligned}
$$

Como $\mathbb{P}=\rho_{P}^{\max }+\rho_{P}^{\min }+\rho_{P}^{m}$ e $\mathbb{S}=\sigma_{P}^{\max }+\sigma_{P}^{\min }+\sigma_{P}^{m}$, substituindo $\mathbb{S}$ por $\sigma_{P}^{\max }+\sigma_{P}^{\min }+\sigma_{P}^{m}$ no 
primeiro somando e $\mathbb{P}$ por $\rho_{P}^{\max }+\rho_{P}^{\min }+\rho_{P}^{m}$ no segundo temos:

$$
\begin{array}{ll} 
& \mathbb{S P} \rho_{P}^{\min }+\mathbb{S P} \sigma_{P}^{\min } \\
\leq & \left(\boldsymbol{\sigma}_{\boldsymbol{P}}^{\max }+\boldsymbol{\sigma}_{P}^{\min }+\boldsymbol{\sigma}_{\boldsymbol{P}}^{m}\right) \mathbb{P} \rho_{P}^{\min }+\mathbb{S}\left(\boldsymbol{\rho}_{\boldsymbol{P}}^{\max }+\boldsymbol{\rho}_{\boldsymbol{P}}^{\min }+\boldsymbol{\rho}_{\boldsymbol{P}}^{\boldsymbol{m}}\right) \sigma_{P}^{\min } \\
& +\mathbb{S}\left(\rho_{P}^{\min }\left(\sigma_{P}^{\max }-\sigma_{P}^{\min }\right)+\rho_{P}^{\min } \sigma_{P}^{m}\right)+\mathbb{P}\left(\sigma_{P}^{\min }\left(\rho_{P}^{\max }-\rho_{P}^{\min }\right)+\sigma_{P}^{\min } \rho_{P}^{m}\right) \\
= & \mathbb{S}\left(\sigma_{P}^{\max } \rho_{P}^{\min }+\rho_{P}^{\max } \sigma_{P}^{\min }+\sigma_{P}^{\min } \rho_{P}^{m}+\rho_{P}^{\min } \sigma_{P}^{m}\right)+\mathbb{P}\left(\sigma_{P}^{\max } \rho_{P}^{\min }+\rho_{P}^{\max } \sigma_{P}^{\min }+\sigma_{P}^{\min } \rho_{P}^{m}+\rho_{P}^{\min }\right) \\
= & (\mathbb{S}+\mathbb{P})\left(\sigma_{P}^{\max } \rho_{P}^{\min }+\rho_{P}^{\min } \sigma_{P}^{\max }+\sigma_{P}^{\min } \rho_{P}^{m}+\rho_{P}^{\min } \sigma_{P}^{m}\right) \\
\leq \quad & (\mathbb{S}+\mathbb{P})\left(\sigma_{P}^{f} \rho_{P}^{l}+\rho_{P}^{f} \sigma_{P}^{l}+\sigma_{P}^{\min } \rho_{P}^{m}+\rho_{P}^{\min } \sigma_{P}^{m}\right) .
\end{array}
$$

A última desigualdade foi dada pelo fato 5.13. Aplicando este resultado no valor de $\phi$ obtemos:

$$
\begin{aligned}
\phi(P) \leq & (\mathbb{S}+\mathbb{P})\left(\sigma_{P}^{f} \rho_{P}^{l}+\rho_{P}^{f} \sigma_{P}^{l}+\sigma_{P}^{\min } \rho_{P}^{m}+\rho_{P}^{\min } \sigma_{P}^{m}\right) \omega(P) \\
& +\frac{\delta}{2}(\mathbb{S}+\mathbb{P})\left(\sigma_{P}^{f} \rho_{P}^{l}+\rho_{P}^{f} \sigma_{P}^{l}+\sigma_{P}^{\min } \rho_{P}^{m}+\rho_{P}^{\min } \sigma_{P}^{m}\right) \omega(P) \\
& +\mathbb{S}\left(\sigma_{P}^{\max }-\sigma_{P}^{\min }\right) \mathbb{P}(P)+\mathbb{P}\left(\rho_{P}^{\max }-\rho_{P}^{\min }\right) \mathbb{S}(P) \\
& +\mathbb{P}\left(\sigma_{P}^{\max }-\sigma_{P}^{\min }\right) \mathbb{S}(P)+\mathbb{S}\left(\rho_{P}^{\max }-\rho_{P}^{\min }\right) \mathbb{P}(P) \\
& -\left(\mathbb{P} \sigma_{P}^{\min } \rho_{P}^{m}+\mathbb{S} \rho_{P}^{\min } \sigma_{P}^{m}\right) \omega(P) .
\end{aligned}
$$

Note que $(5.21)+(5.22)=(5.24)$, e $(5.23)+(5.25)+(5.26)$. Logo:

$$
\begin{aligned}
\phi(P) \leq & \frac{(2+\delta)}{2}(\mathbb{S}+\mathbb{P})\left(\sigma_{P}^{f} \rho_{P}^{l}+\rho_{P}^{f} \sigma_{P}^{l}+\sigma_{P}^{\min } \rho_{P}^{m}+\rho_{P}^{\min } \sigma_{P}^{m}\right) \omega(P) \\
& +(\mathbb{S}+\mathbb{P})\left(\left(\sigma_{P}^{\max }-\sigma_{P}^{\min }\right) \mathbb{P}(P)+\left(\rho_{P}^{\max }-\rho_{P}^{\min }\right) \mathbb{S}(P)\right) \\
& -\mathbb{P} \sigma_{P}^{\max } \mathbb{P}(P)+\mathbb{P} \sigma_{P}^{\min } \mathbb{P}(P)-\mathbb{S} \rho_{P}^{\max } \mathbb{S}(P)+\mathbb{S} \rho_{P}^{\min } \mathbb{S}(P) \\
& +\mathbb{P}\left(\sigma_{P}^{\max }-\sigma_{P}^{\min }\right) \mathbb{S}(P)+\mathbb{S}\left(\rho_{P}^{\max }-\rho_{P}^{\min }\right) \mathbb{P}(P) \\
& -\left(\mathbb{P} \sigma_{P}^{\min } \rho_{P}^{m}+\mathbb{S} \rho_{P}^{\min } \sigma_{P}^{m}\right) \omega(P) .
\end{aligned}
$$

Como $\rho_{P}^{m} \omega(P)=\mathbb{P}^{f}(P)+\mathbb{P}^{l}(P)$ e $\sigma_{P}^{m} \omega(P)=\mathbb{S}^{f}(P)+\mathbb{S}^{l}(P)$, temos que $\rho_{P}^{m} \omega(P) \geq \mathbb{P}(P)$ e $\sigma_{P}^{m} \omega(P) \geq \mathbb{S}(P)$, logo:

$$
\begin{aligned}
\phi(P) \leq & \frac{(2+\delta)}{2}(\mathbb{S}+\mathbb{P})\left(\sigma_{P}^{f} \rho_{P}^{l}+\rho_{P}^{f} \sigma_{P}^{l}+\sigma_{P}^{\min } \rho_{P}^{m}+\rho_{P}^{\min } \sigma_{P}^{m}\right) \omega(P) \\
& +(\mathbb{S}+\mathbb{P})\left(\left(\sigma_{P}^{\max }-\sigma_{P}^{\min }\right) \mathbb{P}(P)+\left(\rho_{P}^{\max }-\rho_{P}^{\min }\right) \mathbb{S}(P)\right) \\
& -\mathbb{P} \sigma_{P}^{\max } \mathbb{P}(P)+\mathbb{P} \boldsymbol{\sigma}_{\boldsymbol{P}}^{\min } \mathbb{P}(\boldsymbol{P})-\mathbb{S} \rho_{P}^{\max } \mathbb{S}(P)+\mathbb{S} \boldsymbol{\rho}_{\boldsymbol{P}}^{\min } \mathbb{S}(\boldsymbol{P}) \\
& +\mathbb{P}\left(\sigma_{P}^{\max }-\sigma_{P}^{\min }\right) \mathbb{S}(P)+\mathbb{S}\left(\rho_{P}^{\max }-\rho_{P}^{\min }\right) \mathbb{P}(P) \\
& -\mathbb{P} \boldsymbol{\sigma}_{\boldsymbol{P}}^{\min } \mathbb{P}(\boldsymbol{P})-\mathbb{S} \boldsymbol{\rho}_{\boldsymbol{P}}^{\min } \mathbb{S}(\boldsymbol{P}) .
\end{aligned}
$$

A soma dos termos não negativos da linha (5.27) com a (5.28) resulta zero, logo:

$$
\begin{aligned}
\phi(P) \leq & \frac{(2+\delta)}{2}(\mathbb{S}+\mathbb{P})\left(\sigma_{P}^{f} \rho_{P}^{l}+\rho_{P}^{f} \sigma_{P}^{l}+\sigma_{P}^{\min } \rho_{P}^{m}+\rho_{P}^{\min } \sigma_{P}^{m}\right) \omega(P) \\
& +(\mathbb{S}+\mathbb{P})\left(\left(\sigma_{P}^{\max }-\sigma_{P}^{\min }\right) \mathbb{P}(P)+\left(\rho_{P}^{\max }-\rho_{P}^{\min }\right) \mathbb{S}(P)\right) \\
& -\mathbb{P} \sigma_{P}^{\max } \mathbb{P}(P)-\mathbb{S} \rho_{P}^{\max ^{2}} \mathbb{S}(P)
\end{aligned}
$$




$$
\begin{aligned}
& +\mathbb{P}\left(\sigma_{P}^{\max }-\sigma_{P}^{\min }\right) \mathbb{S}(P)+\mathbb{S}\left(\rho_{P}^{\max }-\rho_{P}^{\min }\right) \mathbb{P}(P) \\
\leq & \frac{(2+\delta)}{2}(\mathbb{S}+\mathbb{P})\left(\sigma_{P}^{f} \rho_{P}^{l}+\rho_{P}^{f} \sigma_{P}^{l}+\sigma_{P}^{\min } \rho_{P}^{m}+\rho_{P}^{\min } \sigma_{P}^{m}\right) \omega(P) \\
& +(\mathbb{S}+\mathbb{P})\left(\left(\sigma_{P}^{\max }-\sigma_{P}^{\min }\right) \mathbb{P}(P)+\left(\rho_{P}^{\max }-\rho_{P}^{\min }\right) \mathbb{S}(P)\right) \\
& +\left(\mathbb{P} \boldsymbol{\sigma}_{\boldsymbol{P}}^{\max }-\mathbb{P} \boldsymbol{\sigma}_{\boldsymbol{P}}^{\min }-\mathbb{S} \boldsymbol{\rho}_{\boldsymbol{P}}^{\max }\right) \mathbb{S}(\boldsymbol{P})+\left(\mathbb{S} \boldsymbol{\rho}_{\boldsymbol{P}}^{\max }-\mathbb{S} \boldsymbol{\rho}_{\boldsymbol{P}}^{\min }-\mathbb{P} \boldsymbol{\sigma}_{\boldsymbol{P}}^{\max }\right) \mathbb{P}(\boldsymbol{P}) .
\end{aligned}
$$

Agora existem duas possibilidades que são simétricas:

$$
\mathbb{P} \sigma_{P}^{\max } \geq \mathbb{S} \rho_{P}^{\max } \text { ou } \mathbb{P} \sigma_{P}^{\max } \leq \mathbb{S} \rho_{P}^{\max }
$$

A análise de ambos os casos é similar, sendo somente necessário substituir os valores associados com os requerimentos de envios pelos associados com os requerimentos de recepção para transformar um caso no outro; isto é, comutar $\mathbb{S}, \sigma$ e $\mathbb{S}(P)$, respectivamente por $\mathbb{P}, \rho$ e $\mathbb{P}(P)$ (e vice-versa). Logo, vamos mostrar a prova para o caso em que $\mathbb{P} \sigma_{P}^{\max } \geq \mathbb{S} \rho_{P}^{\max }$. O outro caso é análogo.

$$
\begin{aligned}
& \left(\mathbb{P} \sigma_{P}^{\max }-\mathbb{P} \sigma_{P}^{\min }-\mathbb{S} \rho_{P}^{\max }\right) \mathbb{S}(P)+\left(\mathbb{S} \rho_{P}^{\max }-\mathbb{S} \rho_{P}^{\min }-\mathbb{P} \boldsymbol{\sigma}_{P}^{\max }\right) \mathbb{P}(P) \\
\leq \quad & \left(\mathbb{P} \sigma_{P}^{\max }-\mathbb{P} \sigma_{P}^{\min }-\mathbb{S} \rho_{P}^{\max }\right) \mathbb{S}(P) .
\end{aligned}
$$

Como $\rho_{P}^{\max }=\mathbb{P}-\rho_{P}^{\min }-\rho_{P}^{m}$, temos:

$$
\begin{aligned}
& \left(\mathbb{P} \sigma_{P}^{\max }-\mathbb{P} \sigma_{P}^{\min }-\mathbb{S} \rho_{P}^{\max }\right) \mathbb{S}(P) \\
= & \left(\mathbb{P} \sigma_{P}^{\max }-\mathbb{P} \sigma_{P}^{\min }-\mathbb{S}\left(\mathbb{P}-\boldsymbol{\rho}_{P}^{\min }-\boldsymbol{\rho}_{P}^{\boldsymbol{m}}\right)\right) \mathbb{S}(P) \\
= & \left(\mathbb{P}\left(\sigma_{P}^{\max }-\mathbb{S}\right)-\mathbb{P} \sigma_{P}^{\min }+\mathbb{S} \rho_{P}^{\min }+\mathbb{S} \rho_{P}^{m}\right) \mathbb{S}(P)
\end{aligned}
$$

Note que $\sigma_{P}^{\max } \leq \mathbb{S}$, logo:

$$
\begin{aligned}
& \left(\mathbb{P} \sigma_{P}^{\max }-\mathbb{P} \sigma_{P}^{\min }-\mathbb{S} \rho_{P}^{\max }\right) \mathbb{S}(P) \\
\leq \quad & \left(\mathbb{S} \rho_{P}^{\min }+\mathbb{S} \rho_{P}^{m}\right) \mathbb{S}(P) \leq 2 \mathbb{S} \max \left\{\rho_{P}^{\min }, \rho_{P}^{m}\right\} \mathbb{S}(P) .
\end{aligned}
$$

Como $\sigma_{P}^{m} \omega(P) \geq \mathbb{S}(P)$, obtemos:

$$
\begin{aligned}
& \left(\mathbb{P} \sigma_{P}^{\max }-\mathbb{P} \sigma_{P}^{\min }-\mathbb{S} \rho_{P}^{\max }\right) \mathbb{S}(P) \\
\leq \quad & 2 \mathbb{S} \max \left\{\rho_{P}^{\min }, \rho_{P}^{m}\right\} \boldsymbol{\sigma}_{\boldsymbol{P}}^{m} \boldsymbol{\omega}(\boldsymbol{P}) .
\end{aligned}
$$

Agora analisamos dois possíveis casos:

- Caso em que $\rho_{P}^{m}>\rho_{P}^{\min }$, logo:

$$
2 \mathbb{S} \max \left\{\rho_{P}^{\min }, \rho_{P}^{m}\right\} \sigma_{P}^{m} \omega(P)=2 \mathbb{S} \rho_{P}^{m} \sigma_{P}^{m} \omega(P),
$$

como $\sigma_{P}^{m} \leq \frac{\delta}{6} \mathbb{S}$ e $\rho_{P}^{m} \leq \frac{\delta}{6} \mathbb{P}$ :

$$
2 \mathbb{S} \rho_{P}^{m} \sigma_{P}^{m} \omega(P) \leq 2 \mathbb{S} \frac{\delta}{6} \mathbb{P} \frac{\delta}{6} \mathbb{S}
$$


o que implica:

$$
\left(\mathbb{P} \sigma_{P}^{\max }-\mathbb{P} \sigma_{P}^{\min }-\mathbb{S} \rho_{P}^{\max }\right) \mathbb{S}(P)+\left(\mathbb{S} \rho_{P}^{\max }-\mathbb{S} \rho_{P}^{\min }-\mathbb{P} \sigma_{P}^{\max }\right) \mathbb{P}(P) \leq \frac{\delta^{2}}{18} \mathbb{S S P} \omega(P) .
$$

Sem perda de generalidade suponha que $\sigma_{P}^{f}=\sigma_{P}^{\max }$, isto significa que $\sigma_{P}^{l}=\sigma_{P}^{\min }$. Então existem duas possibilidades:

- A primeira é que $\rho_{P}^{f}=\rho_{P}^{\max }$ implicando que $\rho_{P}^{l}=\rho_{P}^{\min }$. Neste caso, como $\rho_{P}^{m}>\rho_{P}^{\min }=\rho_{P}^{l}$ e $\rho_{P}^{m} \leq \frac{\delta}{6} \mathbb{P}$ (por ser $P$ um $\delta$ - $\sigma \rho$-caminho), temos:

$$
\rho_{P}^{m}+\rho_{P}^{l} \leq 2 \rho_{P}^{m} \leq \frac{\delta}{3} \mathbb{P}<\delta \mathbb{P}
$$

Observe que se $\sigma_{P}^{l}=\sigma_{P}^{\min } \leq \frac{\delta}{2} \mathbb{S}$, então, como $\sigma_{P}^{m} \leq \frac{\delta}{6} \mathbb{S}$ :

$$
\sigma_{P}^{m}+\sigma_{P}^{l} \leq \frac{2 \delta}{3} \mathbb{S}<\delta \mathbb{S}
$$

Das inequações (5.29) e (5.30) temos que a sub-árvore de $S$ induzida pelos vértices de $V B\left(S, E_{P}, f_{P}\right)$ (isto é, $\left.S \backslash \bigcup_{v \in V_{P}-f_{P}} V B\left(S, E_{P}, v\right)\right)$ é um $\delta$ - $\sigma \rho$-separador e isto implica que $S$ não pode ser minimal o que é uma contradição, portanto $\sigma_{P}^{\min }>\frac{\delta}{2} \mathbb{S}$. Usando este resultado:

$$
\begin{aligned}
& \left(\mathbb{P} \sigma_{P}^{\max }-\mathbb{P} \sigma_{P}^{\min }-\mathbb{S} \rho_{P}^{\max }\right) \mathbb{S}(P)+\left(\mathbb{S} \rho_{P}^{\max }-\mathbb{S} \rho_{P}^{\min }-\mathbb{P} \sigma_{P}^{\max }\right) \mathbb{P}(P) \\
\leq & \frac{\delta^{2}}{18} \mathbb{S S P} \omega(P) \leq \frac{\delta^{2}}{6} \mathbb{S S P} \omega(P)=\frac{\delta}{3}\left(\frac{\delta}{2} \mathbb{S}\right) \mathbb{S P} \omega(P) \\
\leq & \frac{\delta}{3} \sigma_{P}^{\min } \mathbb{S P} \omega(P)
\end{aligned}
$$

Como $\mathbb{P}=\rho_{P}^{\max }+\rho_{P}^{\min }+\rho_{P}^{m}$, temos:

$$
\begin{aligned}
& \left(\mathbb{P} \sigma_{P}^{\max }-\mathbb{P} \sigma_{P}^{\min }-\mathbb{S} \rho_{P}^{\max }\right) \mathbb{S}(P)+\left(\mathbb{S} \rho_{P}^{\max }-\mathbb{S} \rho_{P}^{\min }-\mathbb{P} \sigma_{P}^{\max }\right) \mathbb{P}(P) \\
\leq & \frac{\delta}{3} \mathbb{S} \sigma_{P}^{\min }\left(\rho_{P}^{\max }+\rho_{P}^{\min }+\rho_{P}^{m}\right) \omega(P) \\
= & \frac{\delta}{3} \mathbb{S}\left(\sigma_{P}^{\min } \rho_{P}^{\max }+\sigma_{P}^{\max } \rho_{P}^{\min }+\sigma_{P}^{\min } \rho_{P}^{m}+\rho_{P}^{\min } \sigma_{P}^{m}\right) \omega(P) \\
\leq & \frac{\delta}{3} \mathbb{S}\left(\sigma_{P}^{f} \rho_{P}^{l}+\sigma_{P}^{l} \rho_{P}^{f}+\sigma_{P}^{\min } \rho_{P}^{m}+\rho_{P}^{\min } \sigma_{P}^{m}\right) \omega(P) .
\end{aligned}
$$

A última desigualdade foi resultado do fato 5.13.

- A outra possibilidade é que $\rho_{P}^{l}=\rho_{P}^{\max }$. Como $2 \rho_{P}^{\max } \geq \mathbb{P}-\rho_{P}^{m} \geq \mathbb{P}-\frac{\delta}{6} \mathbb{P} \geq \frac{11}{12} \mathbb{P}$ e $2 \sigma_{P}^{\max } \geq \mathbb{S}-\sigma_{P}^{m} \geq \mathbb{S}-\frac{\delta}{6} \mathbb{S} \geq \frac{11}{12} \mathbb{S}:$

$$
\begin{aligned}
& \left(\mathbb{P} \sigma_{P}^{\max }-\mathbb{P} \sigma_{P}^{\min }-\mathbb{S} \rho_{P}^{\max }\right) \mathbb{S}(P)+\left(\mathbb{S} \rho_{P}^{\max }-\mathbb{S} \rho_{P}^{\min }-\mathbb{P} \sigma_{P}^{\max }\right) \mathbb{P}(P) \\
\leq & \frac{\delta^{2}}{18} \mathbb{S S P} \omega(P)=\frac{\delta^{2}}{3} \frac{1}{6} \mathbb{S S P} \omega(P) \leq \frac{\delta^{2}}{3} \frac{121}{576} \mathbb{S S P} \omega(P) \\
= & \frac{\delta^{2}}{3}\left(\frac{11}{24} \mathbb{S}\right)\left(\frac{11}{24} \mathbb{P}\right) \mathbb{S} \omega(P) \leq \frac{\delta^{2}}{3} \rho_{P}^{\max } \sigma_{P}^{\max } \mathbb{S} \omega(P)
\end{aligned}
$$




$$
\begin{aligned}
& \leq \frac{\delta}{3} \mathbb{S}\left(\rho_{P}^{\max } \sigma_{P}^{\max }+\rho_{P}^{\min } \sigma_{P}^{\min }+\rho_{P}^{\min } \sigma_{P}^{m}+\sigma_{P}^{\min } \rho_{P}^{m}\right) \omega(P) \\
& =\frac{\delta}{3} \mathbb{S}\left(\rho_{P}^{l} \sigma_{P}^{f}+\rho_{P}^{f} \sigma_{P}^{l}+\rho_{P}^{\min } \sigma_{P}^{m}+\sigma_{P}^{\min } \rho_{P}^{m}\right) \omega(P) .
\end{aligned}
$$

- O outro caso é que $\rho_{P}^{m} \leq \rho_{P}^{\min }$ :

$$
\left(\mathbb{P} \sigma_{P}^{\max }-\mathbb{P} \sigma_{P}^{\min }-\mathbb{S} \rho_{P}^{\max }\right) \mathbb{S}(P)+\left(\mathbb{S} \rho_{P}^{\max }-\mathbb{S} \rho_{P}^{\min }-\mathbb{P} \sigma_{P}^{\max }\right) \mathbb{P}(P) \leq 2 \mathbb{S} \rho_{P}^{\min } \sigma_{P}^{m} \omega(P) .
$$

Como $\sigma_{P}^{m} \leq \frac{\delta}{6} R_{s}$ :

$$
\begin{aligned}
& \left(\mathbb{P} \sigma_{P}^{\max }-\mathbb{P} \sigma_{P}^{\min }-\mathbb{S} \rho_{P}^{\max }\right) \mathbb{S}(P)+\left(\mathbb{S} \rho_{P}^{\max }-\mathbb{S} \rho_{P}^{\min }-\mathbb{P} \sigma_{P}^{\max }\right) \mathbb{P}(P) \\
\leq & \frac{\delta}{3} \mathbb{S} \rho_{P}^{\min } \mathbb{S} \omega(P)=\frac{\delta}{3} \mathbb{S} \rho_{P}^{\min }\left(\sigma_{P}^{\max }+\sigma_{P}^{\min }+\sigma_{P}^{m}\right) \omega(P) \\
\leq & \frac{\delta}{3} \mathbb{S}\left(\rho_{P}^{\min } \sigma_{P}^{\max }+\rho_{P}^{\max } \sigma_{P}^{\min }+\rho_{P}^{\min } \sigma_{P}^{m}+\sigma_{P}^{\min } \rho_{P}^{m}\right) \omega(P) \\
\leq & \frac{\delta}{3} \mathbb{S}\left(\rho_{P}^{f} \sigma_{P}^{l}+\rho_{P}^{l} \sigma_{P}^{f}+\rho_{P}^{\min } \sigma_{P}^{m}+\sigma_{P}^{\min } \rho_{P}^{m}\right) \omega(P) .
\end{aligned}
$$

Portanto, qualquer seja o caso:

$$
\begin{aligned}
& \left(\mathbb{P} \sigma_{P}^{\max }-\mathbb{P} \sigma_{P}^{\min }-\mathbb{S} \rho_{P}^{\max }\right) \mathbb{S}(P)+\left(\mathbb{S} \rho_{P}^{\max }-\mathbb{S} \rho_{P}^{\min }-\mathbb{P} \sigma_{P}^{\max }\right) \mathbb{P}(P) \\
\leq & \frac{\delta}{3}(\mathbb{S}+\mathbb{P})\left(\rho_{P}^{f} \sigma_{P}^{l}+\rho_{P}^{l} \sigma_{P}^{f}+\rho_{P}^{\min } \sigma_{P}^{m}+\sigma_{P}^{\min } \rho_{P}^{m}\right) \omega(P) .
\end{aligned}
$$

Concluindo que:

$$
\begin{aligned}
\phi(P) \leq & \frac{6+5 \delta}{6}(\mathbb{S}+\mathbb{P})\left(\sigma_{P}^{f} \rho_{P}^{l}+\sigma_{P}^{l} \rho_{P}^{f}+\sigma_{P}^{\min } \rho_{P}^{m}+\sigma_{P}^{m} \rho_{P}^{\min }\right) \omega(P) \\
& +(\mathbb{S}+\mathbb{P})\left(\left(\sigma_{P}^{\max }-\sigma_{P}^{\min }\right) \mathbb{P}(P)+\left(\rho_{P}^{\max }-\rho_{P}^{\min }\right) \mathbb{S}(P)\right) .
\end{aligned}
$$

\subsection{Limite superior para o centro}

Nesta seção provamos a proposição 5.11, em que dado um $\delta$ - $\sigma \rho$-spine $Y$ de uma árvore geradora $T$ de $G$, provamos a existência de um $|\operatorname{ext}(Y)|$-centro $X$ de $G$ com custo de comunicação $C(X)$ limitado superiormente por:

$$
\begin{aligned}
& \sum_{P \in Y}\left(\sigma_{P}^{f} \rho_{P}^{l}+\rho_{P}^{f} \sigma_{P}^{l}\right) \omega(P)+\min \left\{\Delta_{f l}(P), \Delta_{l f}(P)\right\} \\
& +\mathbb{P} \sum_{u \in V_{G}} \sigma(u) d(T, u, S)+\mathbb{S} \sum_{u \in V_{G}} \rho(u) d(T, u, S),
\end{aligned}
$$

$\operatorname{com} \Delta_{w z}(P)=\omega(P)\left(\sigma_{P}^{m} \rho_{P}^{w}+\rho_{P}^{m} \sigma_{P}^{w}\right)+\mathbb{P S}^{z}(P)+\mathbb{S P}^{z}(P), w, z \in\{f, l\}$, e $S=\bigcup_{P \in Y} P$.

Demonstração. Considere $S=\cup_{P \in Y} P$ o $\delta$ - $\sigma \rho$-separador minimal de $T$ associado a $Y$, então definimos o $|\operatorname{ext}(Y)|$-centro $X$ de $G$ como segue: 
- O esqueleto $\tau$ de $X$ tem como conjunto de vértices aqueles que são extremos dos caminhos em $Y$, isto é, o conjunto $\operatorname{ext}(Y)$. As arestas de $\tau$ são definidas em função dos caminhos em $Y$, de forma tal que dois vértices $u, v \in \tau$ são adjacentes em $\tau$ se existe um caminho em $Y$ do qual $u$ e $v$ são pontos extremos. Observe que por definição todos os caminhos em $Y$ são internamente disjuntos dois a dois e a união deles é a sub-árvore $S$ de $T$, logo, $\tau$ é uma árvore sobre os vértices em $\operatorname{ext}(Y)$.

- Para garantir que $X$ seja um $|\operatorname{ext}(Y)|$-centro com esqueleto $\tau$, cada vértice de $G \backslash \tau$ deve ser adicionado como uma folha de $X$ que será adjacente de algum vértice de $\tau$. A seguir descrevemos como esta adição é feita para um vértice $u \in V_{G \backslash \tau}$ :

- Se existe um vértice $v$ de $\tau$ tal que $u \in V\left(T, E_{S}, v\right)$, então adicionamos a aresta $(u, v)$ em $X$.

- Caso contrário temos que existe um caminho $P \in Y$ tal que $u \in V \backslash V\left(T, E_{S}, f_{P}\right) \backslash$ $V\left(T, E_{S}, l_{P}\right)$, note que $f_{P}$ e $l_{P}$ pertencem a $\tau$. Logo, se $\Delta_{f l}(P) \leq \Delta_{l f}(P)$, então incluímos a aresta $\left(u, f_{P}\right)$ em $X$, caso contrário incluímos em $X$ a aresta $\left(u, l_{P}\right)$.

A Figura 5.2 mostra o exemplo de um spine de uma árvore geradora $T$ de um grafo $G$, e a Figura 5.3 mostra o centro de $G$ obtido a partir desse spine. Formalmente definimos $X$ :

- $V_{X}=V_{G}$

- $E_{\tau}=\{(u, v) \mid \exists P \in Y$ com extremos $u$ e $v\}$

- $E_{X}=E_{\tau} \cup\left\{(u, v) \mid u \in V_{\tau}\right.$ e $\left.v \in V\left(T, E_{S}, u\right)\right\}$

$$
\begin{aligned}
& \cup\left\{(u, v) \mid \exists P \in Y: v \in V(T, P) \text { e } g(P)=1 \text { e } u=f_{P}\right\} \\
& \cup\left\{(u, v) \mid \exists P \in Y: v \in V(T, P) \text { e } g(P)=0 \text { e } u=l_{P}\right\}
\end{aligned}
$$

onde, $V(T, P)=\bigcup_{u \in V_{P} \backslash\{f, l\}} V\left(T, E_{P}, u\right)$, e $g(P)=1$ se $\Delta_{f l}(P) \leq \Delta_{l f}(P)$, caso contrário $g(P)=0$.

Esta construção garante que $X$ seja um $|\operatorname{ext}(Y)|$-centro de $G$ com esqueleto $\tau$. Note que se $u$ é uma folha de $\tau$ e $\left\{v_{1}, \cdots, v_{r}\right\}$ é o conjunto de folhas adjacentes a $u$ em $X$, então $\sigma(u)+\sum_{i=1}^{r} \sigma\left(v_{i}\right)>$ $\delta \mathbb{S}$ ou $\rho(u)+\sum_{i=1}^{r} \rho\left(v_{i}\right)>\delta \mathbb{P}$, pois caso contrário $S$ não seria um $\delta$ - $\sigma \rho$-separador minimal de $T$. Logo, temos que $X$ é $\delta$-balanceado sobre $\tau$, faltando agora analisar o custo de comunicação.

Como toda aresta é um caminho com dois vértices, se expressamos o custo de comunicação de $X$ como a soma do total de requerimento que passa por cada resta em $X$ multiplicado pelo comprimento da aresta:

$$
C(X)=\sum_{e \in E_{X}}\left(\sigma\left(V\left(X, e, f_{e}\right)\right) \rho\left(V\left(X, e, l_{e}\right)\right)+\rho\left(V\left(X, e, f_{e}\right)\right) \sigma\left(V\left(X, e, l_{e}\right)\right)\right) \omega(e)
$$

É possível dividir essa expressão nas arestas em $\tau$ e naquelas fora de $\tau$ :

$$
\begin{aligned}
C(X)= & \sum_{e \in E_{\tau}}\left(\sigma\left(V\left(X, e, f_{e}\right)\right) \rho\left(V\left(X, e, l_{e}\right)\right)+\rho\left(V\left(X, e, f_{e}\right)\right) \sigma\left(V\left(X, e, l_{e}\right)\right)\right) \omega(e) \\
& +\sum_{e \in E_{X \backslash \tau}}\left(\sigma\left(V\left(X, e, f_{e}\right)\right) \rho\left(V\left(X, e, l_{e}\right)\right)+\rho\left(V\left(X, e, f_{e}\right)\right) \sigma\left(V\left(X, e, l_{e}\right)\right)\right) \omega(e) .
\end{aligned}
$$




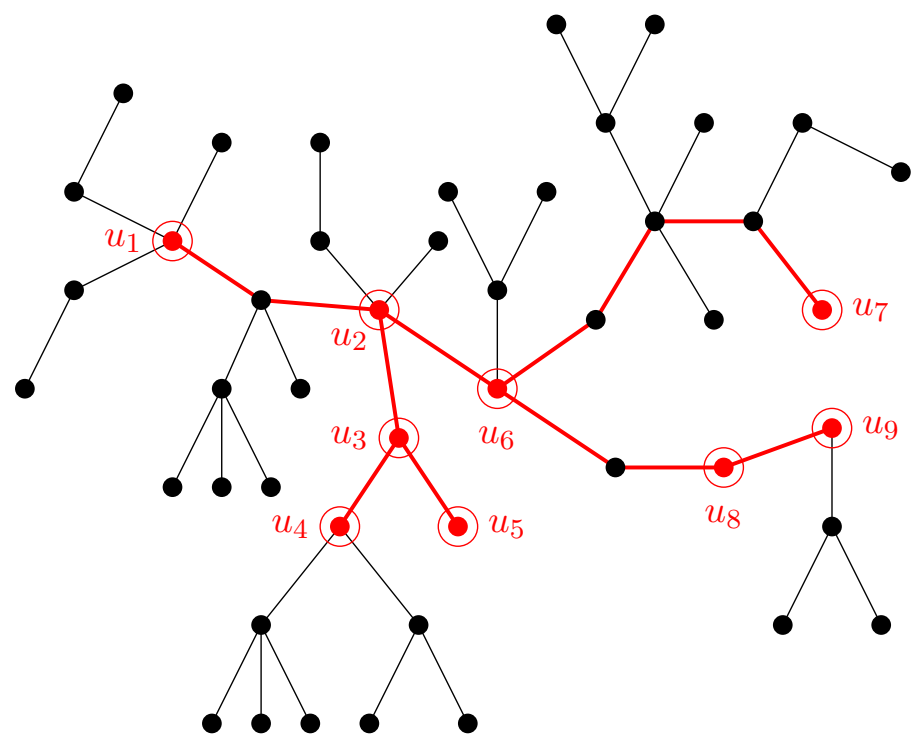

Figura 5.2: Exemplo de um spine de uma árvore geradora de G. No exemplo o spine tem 8 caminhos, cujos extremos são os vértices $u_{1}, u_{2}, u_{3}, u_{4}, u_{5}, u_{6}, u_{7}, u_{8}$ e $u_{9}$ (com uma circunferência ao redor). Assim temos no spine os caminhos entre $u_{1}$ e $u_{2}, u_{2}$ e $u_{3}, u_{3}$ e $u_{4}, u_{3}$ e $u_{5}, u_{2}$ e $u_{6}, u_{6}$ e $u_{7}, u_{6}$ e $u_{8}$, e, $u_{8}$ e $u_{9}$.

Observe que para cada aresta $e \in E_{X \backslash \tau}$ um dos extremos é uma folha de $X$. Se denotamos por $x_{e}$ a folha adjacente de $e \in E_{X \backslash \tau}$, então o custo de comunicação que passa por $e$ é igual a:

$$
\begin{aligned}
\left(\mathbb{S}-\sigma\left(x_{e}\right)\right) \rho\left(x_{e}\right)+\sigma\left(x_{e}\right)\left(\mathbb{P}-\rho\left(x_{e}\right)\right) & =\mathbb{S} \rho\left(x_{e}\right)+\mathbb{P} \sigma\left(x_{e}\right)-2 \sigma\left(x_{e}\right) \rho\left(x_{e}\right) \\
& \leq \mathbb{S} \rho\left(x_{e}\right)+\mathbb{P} \sigma\left(x_{e}\right) .
\end{aligned}
$$

Aplicando esta expressão no custo de comunicação de $X$ obtemos:

$$
\begin{aligned}
C(X) \leq & \sum_{e \in E_{\tau}}\left(\sigma\left(V\left(X, e, f_{e}\right)\right) \rho\left(V\left(X, e, l_{e}\right)\right)+\rho\left(V\left(X, e, f_{e}\right)\right) \sigma\left(V\left(X, e, l_{e}\right)\right)\right) \omega(e) \\
& +\sum_{\boldsymbol{e} \in \boldsymbol{E}_{\boldsymbol{X} \backslash \boldsymbol{\tau}}}\left(\mathbb{S} \boldsymbol{\rho}\left(\boldsymbol{x}_{\boldsymbol{e}}\right)+\mathbb{P} \boldsymbol{\sigma}\left(\boldsymbol{x}_{\boldsymbol{e}}\right)\right) \omega(e) .
\end{aligned}
$$

Note que qualquer aresta $e \in E_{X-\tau}$ satisfaz $d\left(X, x_{e}, \tau\right)=\omega(e)$, logo:

$$
\begin{aligned}
C(X) \leq & \sum_{e \in E_{\tau}}\left(\sigma\left(V\left(X, e, f_{e}\right)\right) \rho\left(V\left(X, e, l_{e}\right)\right)+\rho\left(V\left(X, e, f_{e}\right)\right) \sigma\left(V\left(X, e, l_{e}\right)\right)\right) \omega(e) \\
& +\sum_{e \in E_{X \backslash \tau}}\left(\mathbb{S} \rho\left(x_{e}\right)+\mathbb{P} \sigma\left(x_{e}\right)\right) \boldsymbol{d}\left(\boldsymbol{X}, \boldsymbol{x}_{\boldsymbol{e}}, \boldsymbol{\tau}\right) .
\end{aligned}
$$

Se denotamos por $p\left(x_{e}\right)$ o vértice em $\tau$ adjacente à aresta $e \in E_{X \backslash \tau}$, então $d\left(X, x_{e}, \tau\right)=$ $d\left(X, x_{e}, p\left(x_{e}\right)\right)=\omega(e)$. Como $G$ é métrico, pela desigualdade triangular temos que $d\left(X, x_{e}, \tau\right) \leq$ $d\left(T, x_{e}, p\left(x_{e}\right)\right)$, o que implica:

$$
\begin{aligned}
C(X) \leq & \sum_{e \in E_{\tau}}\left(\sigma\left(V\left(X, e, f_{e}\right)\right) \rho\left(V\left(X, e, l_{e}\right)\right)+\rho\left(V\left(X, e, f_{e}\right)\right) \sigma\left(V\left(X, e, l_{e}\right)\right)\right) \omega(e) \\
& +\sum_{e \in E_{X \backslash \tau}}\left(\mathbb{S} \rho\left(x_{e}\right)+\mathbb{P} \sigma\left(x_{e}\right)\right) \boldsymbol{d}\left(\boldsymbol{T}, \boldsymbol{x}_{\boldsymbol{e}}, \boldsymbol{p}\left(\boldsymbol{x}_{\boldsymbol{e}}\right)\right) .
\end{aligned}
$$




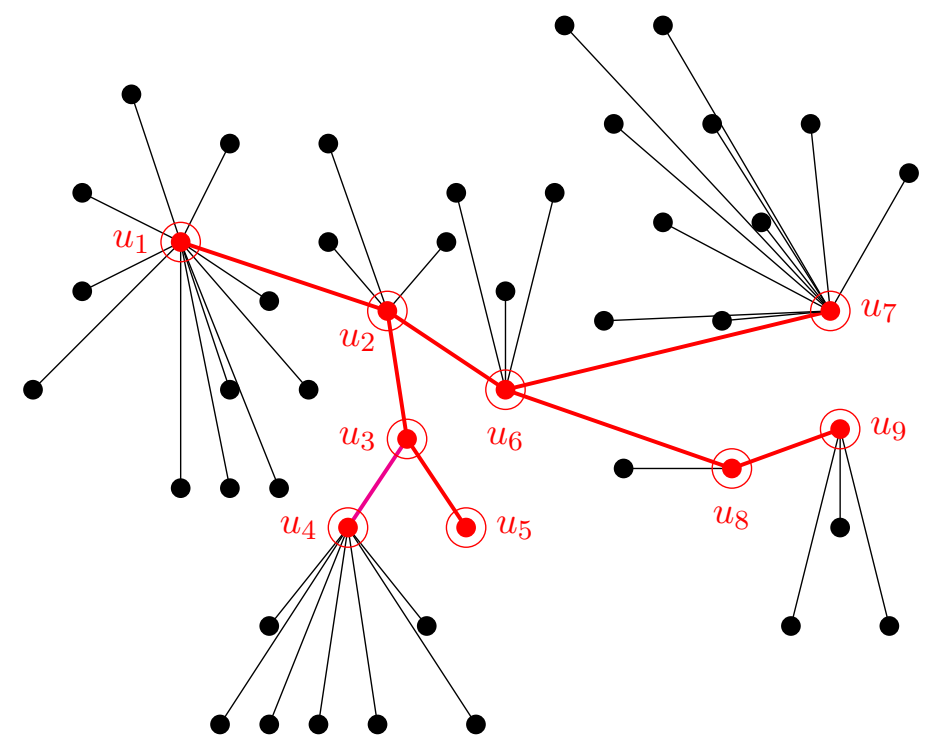

Figura 5.3: Usando o mesmo spine da Figura 5.2, mostramos o centro que obtemos a partir dele. Observe que os vértices conectados ao spine através de um extremos dos caminhos são colocados como adjacentes desse extremo, enquanto os vértices conectados ao spine através de um vértice interno de um dos caminhos (incluindo o próprio vértice interno) são colocados como adjacentes de um dos extremos do caminho. Finalmente se dois vértices são extremos de um caminho no spine, então o caminho é substituído por uma aresta.

Como todo vértice de $G \backslash \tau$ é uma folha adjacente a uma aresta em $X \backslash \tau$ e toda aresta em $X \backslash \tau$ é adjacente a um vértice de $G \backslash \tau$, temos:

$$
\begin{aligned}
C(X) \leq & \sum_{e \in E_{\tau}}\left(\sigma\left(V\left(X, e, f_{e}\right)\right) \rho\left(V\left(X, e, l_{e}\right)\right)+\rho\left(V\left(X, e, f_{e}\right)\right) \sigma\left(V\left(X, e, l_{e}\right)\right)\right) \omega(e) \\
& +\sum_{\boldsymbol{u} \in \boldsymbol{V}_{\boldsymbol{G} \backslash \boldsymbol{\tau}}}(\mathbb{S} \rho(\boldsymbol{u})+\mathbb{P} \sigma(\boldsymbol{u})) d(T, \boldsymbol{u}, p(\boldsymbol{u})) .
\end{aligned}
$$

Definindo por $\partial(T, S, u, v)$ a soma dos comprimentos da arestas de $S$ que pertencem ao caminho entre os vértices $u \in V_{T}$ e $v \in V_{S}$ em $T$, a distância entre dois vértices em $T$ fica:

$$
d(T, u, v)=d(T, u, S)+\partial(T, S, u, v) .
$$

Logo:

$$
\begin{aligned}
C(X) \leq & \sum_{e \in E_{\tau}}\left(\sigma\left(V\left(X, e, f_{e}\right)\right) \rho\left(V\left(X, e, l_{e}\right)\right)+\rho\left(V\left(X, e, f_{e}\right)\right) \sigma\left(V\left(X, e, l_{e}\right)\right)\right) \omega(e) \\
& +\sum_{u \in V_{G \backslash \tau}}(\mathbb{S} \rho(u)+\mathbb{P} \sigma(u))(\boldsymbol{d}(\boldsymbol{T}, \boldsymbol{u}, \boldsymbol{S})+\boldsymbol{\partial}(\boldsymbol{T}, \boldsymbol{S}, \boldsymbol{u}, \boldsymbol{p}(\boldsymbol{u}))) \\
\leq & \sum_{e \in E_{\tau}}\left(\sigma\left(V\left(X, e, f_{e}\right)\right) \rho\left(V\left(X, e, l_{e}\right)\right)+\rho\left(V\left(X, e, f_{e}\right)\right) \sigma\left(V\left(X, e, l_{e}\right)\right)\right) \omega(e) \\
& +\sum_{u \in V_{G \backslash \tau}}(\mathbb{S} \rho(u)+\mathbb{P} \sigma(u)) \boldsymbol{d}(\boldsymbol{T}, \boldsymbol{u}, \boldsymbol{S}) \\
& +\sum_{u \in V_{G \backslash \tau}}(\mathbb{S} \rho(u)+\mathbb{P} \sigma(u)) \boldsymbol{\partial}(\boldsymbol{T}, \boldsymbol{S}, \boldsymbol{u}, \boldsymbol{p}(\boldsymbol{u})) .
\end{aligned}
$$

Como para todo vértice $u \in V_{G \backslash \tau}$ se satisfaz que $p(u) \in V_{\tau} \subseteq V_{S}$ e que $u \in V(T, P)$ onde $P$ é 
algum caminho de $Y$, temos: ${ }^{2}$

$$
\begin{aligned}
C(X) \leq & \sum_{e \in E_{\tau}}\left(\sigma\left(V\left(X, e, f_{e}\right)\right) \rho\left(V\left(X, e, l_{e}\right)\right)+\rho\left(V\left(X, e, f_{e}\right)\right) \sigma\left(V\left(X, e, l_{e}\right)\right)\right) \omega(e) \\
& +\sum_{u \in V_{G \backslash \tau}}(\mathbb{S} \rho(u)+\mathbb{P} \sigma(u)) d(T, u, S) \\
& +\sum_{\boldsymbol{P} \in \boldsymbol{Y}} \sum_{\boldsymbol{u} \in \boldsymbol{V}(\boldsymbol{T}, \boldsymbol{P})}(\mathbb{S} \boldsymbol{\rho}(\boldsymbol{u})+\mathbb{P} \boldsymbol{\sigma}(\boldsymbol{u})) \boldsymbol{\partial}(\boldsymbol{T}, \boldsymbol{S}, \boldsymbol{u}, \boldsymbol{p}(\boldsymbol{u})) .
\end{aligned}
$$

Observe que para $P \in Y$ se $\Delta_{f l}(P) \leq \Delta_{l f}(P)$ então para cada vértice $u \in V(T, P)$ temos que $p(u)=f_{P}$ e $g(P)=1$, caso contrário $p(u)=l_{P}$ para cada $u \in V(T, P)$ e $g(P)=0$, logo para cada $u \in V(T, P):$

$$
\partial(T, S, u, p(u))=g(P) \partial\left(T, S, u, f_{P}\right)+(1-g(P)) \partial\left(T, S, u, l_{P}\right) .
$$

Isto implica:

$$
\begin{aligned}
& C(X) \leq \sum_{e \in E_{\tau}}\left(\sigma\left(V\left(X, e, f_{e}\right)\right) \rho\left(V\left(X, e, l_{e}\right)\right)+\rho\left(V\left(X, e, f_{e}\right)\right) \sigma\left(V\left(X, e, l_{e}\right)\right)\right) \omega(e) \\
& +\sum_{u \in V_{G \backslash \tau}}(\mathbb{S} \rho(u)+\mathbb{P} \sigma(u)) d(T, u, S) \\
& +\sum_{P \in Y} \sum_{u \in V(T, P)}(\mathbb{S} \rho(u)+\mathbb{P} \sigma(u))\left(\boldsymbol{g}(\boldsymbol{P}) \boldsymbol{\partial}\left(\boldsymbol{T}, \boldsymbol{S}, \boldsymbol{u}, \boldsymbol{f}_{\boldsymbol{P}}\right)+(\mathbf{1}-\boldsymbol{g}(\boldsymbol{P})) \boldsymbol{\partial}\left(\boldsymbol{T}, \boldsymbol{S}, \boldsymbol{u}, \boldsymbol{l}_{\boldsymbol{P}}\right)\right) \\
& \leq \sum_{e \in E_{\tau}}\left(\sigma\left(V\left(X, e, f_{e}\right)\right) \rho\left(V\left(X, e, l_{e}\right)\right)+\rho\left(V\left(X, e, f_{e}\right)\right) \sigma\left(V\left(X, e, l_{e}\right)\right)\right) \omega(e) \\
& +\sum_{u \in V_{G \backslash \tau}}(\mathbb{S} \rho(u)+\mathbb{P} \sigma(u)) d(T, u, S) \\
& +\mathbb{S} \sum_{P \in Y} \sum_{u \in V(T, P)} \rho(u)\left(g(P) \partial\left(T, S, u, f_{P}\right)+(1-g(P)) \partial\left(T, S, u, l_{P}\right)\right) \\
& +\mathbb{P} \sum_{P \in Y} \sum_{u \in V(T, P)} \sigma(u)\left(g(P) \partial\left(T, S, u, f_{P}\right)+(1-g(P)) \partial\left(T, S, u, l_{P}\right)\right) \\
& \leq \sum_{e \in E_{\tau}}\left(\sigma\left(V\left(X, e, f_{e}\right)\right) \rho\left(V\left(X, e, l_{e}\right)\right)+\rho\left(V\left(X, e, f_{e}\right)\right) \sigma\left(V\left(X, e, l_{e}\right)\right)\right) \omega(e) \\
& +\sum_{u \in V_{G \backslash \tau}}(\mathbb{S} \rho(u)+\mathbb{P} \sigma(u)) d(T, u, S) \\
& +\mathbb{S} \sum_{P \in Y} g(P) \sum_{u \in V(T, P)} \rho(u) \partial\left(T, S, u, f_{P}\right) \\
& +\mathbb{S} \sum_{P \in Y}(1-g(P)) \sum_{u \in V(T, P)} \rho(u) \partial\left(T, S, u, l_{P}\right) \\
& +\mathbb{P} \sum_{P \in Y} g(P) \sum_{u \in V(T, P)} \sigma(u) \partial\left(T, S, u, f_{P}\right) \\
& +\mathbb{P} \sum_{P \in Y}(1-g(P)) \sum_{u \in V(T, P)} \sigma(u) \partial\left(T, S, u, l_{P}\right) .
\end{aligned}
$$

Note que $\mathbb{P}^{f}(P)=\sum_{u \in V(T, P)} \rho(u) \partial\left(T, S, u, f_{P}\right), \mathbb{P}^{l}(P)=\sum_{u \in V(T, P)} \rho(u) \partial\left(T, S, u, l_{P}\right), \mathbb{S}^{f}(P)=$

\footnotetext{
${ }^{2}$ Lembrando que $V(T, P)=\bigcup_{u \in V_{P}-\left\{f_{P}, l_{P}\right\}} V\left(T, E_{P}, u\right)$.
} 
$\sum_{u \in V(T, P)} \sigma(u) \partial\left(T, S, u, f_{P}\right), \mathbb{S}^{l}(P)=\sum_{u \in V(T, P)} \sigma(u) \partial\left(T, S, u, l_{P}\right)$. Logo:

$$
\begin{aligned}
C(X) \leq & \sum_{e \in E_{\tau}}\left(\sigma\left(V\left(X, e, f_{e}\right)\right) \rho\left(V\left(X, e, l_{e}\right)\right)+\rho\left(V\left(X, e, f_{e}\right)\right) \sigma\left(V\left(X, e, l_{e}\right)\right)\right) \omega(e) \\
& +\sum_{u \in V_{G \backslash \tau}}(\mathbb{S} \rho(u)+\mathbb{P} \sigma(u)) d(T, u, S) \\
& +\mathbb{S} \sum_{P \in Y} g(P) \mathbb{P}^{f}(\boldsymbol{P})+\mathbb{S} \sum_{P \in Y}(1-g(P)) \mathbb{P}^{l}(\boldsymbol{P}) \\
& +\mathbb{P}_{P \in Y} g(P) \mathbb{S}^{f}(\boldsymbol{P})+\mathbb{P} \sum_{P \in Y}(1-g(P)) \mathbb{S}^{\boldsymbol{l}}(\boldsymbol{P}) .
\end{aligned}
$$

Considerando uma aresta $e \in E_{\tau}$, temos que existe um caminho $P \in Y$ com extremos $f_{P}=f_{e}$ e $l_{P}=l_{e}$. Isto implica que se $\Delta_{f l}(P) \leq \Delta_{l f}(P)$, então $g(P)=1$ e:

$$
\begin{aligned}
\sigma\left(V\left(X, e, f_{e}\right)\right) & =\sigma_{P}^{f}+\sigma_{P}^{m} \\
\sigma\left(V\left(X, e, l_{e}\right)\right) & =\sigma_{P}^{l} \\
\rho\left(V\left(X, e, f_{e}\right)\right) & =\rho_{P}^{f}+\rho_{P}^{m} \\
\rho\left(V\left(X, e, l_{e}\right)\right) & =\rho_{P}^{l},
\end{aligned}
$$

caso contrário, $g(P)=0$ e:

$$
\begin{aligned}
\sigma\left(V\left(X, e, f_{e}\right)\right) & =\sigma_{P}^{f} \\
\sigma\left(V\left(X, e, l_{e}\right)\right) & =\sigma_{P}^{l}+\sigma_{P}^{m} \\
\rho\left(V\left(X, e, f_{e}\right)\right) & =\rho_{P}^{f} \\
\rho\left(V\left(X, e, l_{e}\right)\right) & =\rho_{P}^{l}+\rho_{P}^{m} .
\end{aligned}
$$

Isto implica que para cada aresta $e \in E_{\tau}$ existe um caminho $P \in Y$ tal que:

$$
\begin{aligned}
\sigma\left(V\left(X, e, f_{e}\right)\right) & =\sigma_{P}^{f}+g(P) \sigma_{P}^{m} \\
\sigma\left(V\left(X, e, l_{e}\right)\right) & =\sigma_{P}^{l}+(1-g(P)) \sigma_{P}^{m} \\
\rho\left(V\left(X, e, f_{e}\right)\right) & =\rho_{P}^{f}+g(P) \rho_{P}^{m} \\
\rho\left(V\left(X, e, l_{e}\right)\right) & =\rho_{P}^{l}+(1-g(P)) \rho_{P}^{m},
\end{aligned}
$$

logo:

$$
\begin{aligned}
& \sigma\left(V\left(X, e, f_{e}\right)\right) \rho\left(V\left(X, e, l_{e}\right)\right)+\rho\left(V\left(X, e, f_{e}\right)\right) \sigma\left(V\left(X, e, l_{e}\right)\right) \\
= & \left(\sigma_{P}^{f}+g(P) \sigma_{P}^{m}\right)\left(\rho_{P}^{l}+(1-g(P)) \rho_{P}^{m}\right)+\left(\sigma_{P}^{l}+(1-g(P)) \sigma_{P}^{m}\right)\left(\rho_{P}^{f}+g(P) \rho_{P}^{m}\right) \\
=\quad & \sigma_{P}^{f} \rho_{P}^{l}+\sigma_{P}^{l} \rho_{P}^{f}+g(P)\left(\sigma_{P}^{m} \rho_{P}^{l}+\sigma_{P}^{l} \rho_{P}^{m}\right)+(1-g(P))\left(\sigma_{P}^{f} \rho_{P}^{m}+\sigma_{P}^{m} \rho_{P}^{f}\right) .
\end{aligned}
$$

Além, por ser $G$ métrico, para cada aresta $e \in E_{\tau}$ se satisfaz que $\omega(e) \leq \omega(P)$ sendo $P$ o caminho em $Y$ com os mesmos extremos que os de $e$. Logo:

$$
C(X) \leq \sum_{P \in Y}\left(\sigma_{P}^{f} \rho_{P}^{l}+\sigma_{P}^{l} \rho_{P}^{f}\right) \omega(P)
$$




$$
\begin{aligned}
& +\sum_{\boldsymbol{P} \in \boldsymbol{Y}}\left(\boldsymbol{g}(\boldsymbol{P})\left(\boldsymbol{\sigma}_{\boldsymbol{P}}^{\boldsymbol{m}} \boldsymbol{\rho}_{\boldsymbol{P}}^{\boldsymbol{l}}+\boldsymbol{\sigma}_{\boldsymbol{P}}^{\boldsymbol{l}} \boldsymbol{\rho}_{\boldsymbol{P}}^{\boldsymbol{m}}\right)+(\mathbf{1}-\boldsymbol{g}(\boldsymbol{P}))\left(\boldsymbol{\sigma}_{\boldsymbol{P}}^{\boldsymbol{f}} \boldsymbol{\rho}_{\boldsymbol{P}}^{\boldsymbol{m}}+\boldsymbol{\sigma}_{\boldsymbol{P}}^{\boldsymbol{m}} \boldsymbol{\rho}_{\boldsymbol{P}}^{\boldsymbol{f}}\right)\right) \boldsymbol{\omega}(\boldsymbol{P}) \\
& +\sum_{u \in V_{G \backslash \tau}}(\mathbb{S} \rho(u)+\mathbb{P} \sigma(u)) d(T, u, S) \\
& +\mathbb{S} \sum_{P \in Y} g(P) \mathbb{P}^{f}(P)+\mathbb{S} \sum_{P \in Y}(1-g(P)) \mathbb{P}^{l}(P) \\
& +\mathbb{P} \sum_{P \in Y} g(P) \mathbb{S}^{f}(P)+\mathbb{P} \sum_{P \in Y}(1-g(P)) \mathbb{S}^{l}(P) \\
\leq & \sum_{P \in Y}\left(\sigma_{P}^{f} \rho_{P}^{l}+\sigma_{P}^{l} \rho_{P}^{f}\right) \omega(P) \\
& +\sum_{\boldsymbol{P} \in \boldsymbol{Y}} \boldsymbol{g}(\boldsymbol{P})\left(\left(\boldsymbol{\sigma}_{\boldsymbol{P}}^{\boldsymbol{m}} \boldsymbol{\rho}_{\boldsymbol{P}}^{\boldsymbol{l}}+\boldsymbol{\sigma}_{\boldsymbol{P}}^{\boldsymbol{l}} \boldsymbol{\rho}_{\boldsymbol{P}}^{\boldsymbol{m}}\right) \boldsymbol{\omega}(\boldsymbol{P})+\mathbb{S P}^{\boldsymbol{f}}(\boldsymbol{P})+\mathbb{P S}^{\boldsymbol{f}}(\boldsymbol{P})\right) \\
& +\sum_{\boldsymbol{P} \in \boldsymbol{Y}}(\mathbf{1}-\boldsymbol{g}(\boldsymbol{P}))\left(\left(\boldsymbol{\sigma}_{\boldsymbol{P}}^{\boldsymbol{f}} \boldsymbol{\rho}_{\boldsymbol{P}}^{\boldsymbol{m}}+\boldsymbol{\sigma}_{\boldsymbol{P}}^{\boldsymbol{m}} \boldsymbol{\rho}_{\boldsymbol{P}}^{\boldsymbol{f}}+\mathbb{S P}^{\boldsymbol{l}}(\boldsymbol{P})+\mathbb{P S}^{\boldsymbol{l}}(\boldsymbol{P})\right) \boldsymbol{\omega}(\boldsymbol{P})\right) \\
& +\sum_{u \in V_{G}-\tau}(\mathbb{S} \rho(u)+\mathbb{P} \sigma(u)) d(T, u, S) \\
\leq & \sum_{P \in Y}\left(\sigma_{P}^{f} \rho_{P}^{l}+\sigma_{P}^{l} \rho_{P}^{f}\right) \omega(P)+\boldsymbol{g}(\boldsymbol{P}) \boldsymbol{\Delta}_{\boldsymbol{f l}}(\boldsymbol{P})+(\mathbf{1}-\boldsymbol{g}(\boldsymbol{P})) \boldsymbol{\Delta}_{l \boldsymbol{f}}(\boldsymbol{P}) \\
& +\sum_{\boldsymbol{u} \in \boldsymbol{V}_{\boldsymbol{G}}}(\mathbb{S} \rho(u)+\mathbb{P} \sigma(u)) d(T, u, S) .
\end{aligned}
$$

Observe que se $\Delta_{f l}(P) \leq \Delta_{l f}(P)$ então $g(P)=1$, caso contrário $g(P)=0$, portanto:

$$
\begin{aligned}
C(X) \leq & \sum_{P \in Y}\left(\sigma_{P}^{f} \rho_{P}^{l}+\sigma_{P}^{l} \rho_{P}^{f}\right) \omega(P)+\min \left\{\boldsymbol{\Delta}_{\boldsymbol{f} \boldsymbol{l}}(\boldsymbol{P}), \boldsymbol{\Delta}_{\boldsymbol{l f}}(\boldsymbol{P})\right\} \\
& +\sum_{\boldsymbol{u} \in \boldsymbol{V}_{\boldsymbol{G}}}(\mathbb{S} \rho(u)+\mathbb{P} \sigma(u)) d(T, u, S) .
\end{aligned}
$$

\subsection{Limite inferior de uma árvore geradora}

Nesta seção provamos a proposição 5.12, em que dados uma árvore geradora $T$ de $G$ e um $\delta$ - $\sigma \rho$ spine $Y$ de $T$, é possível verificar que $C(T)$, o custo de comunicação de $T$, é limitado inferiormente por:

$$
\begin{aligned}
& \sum_{P \in Y}\left(\rho_{P}^{l} \sigma_{P}^{f}+\rho_{P}^{f} \sigma_{P}^{l}+\rho_{P}^{\min } \sigma_{P}^{m}+\sigma_{P}^{\min } \rho_{P}^{m}\right) \omega(P) \\
& +\sum_{P \in Y}\left(\sigma_{P}^{\max }-\sigma_{P}^{\min }\right) \mathbb{P}(P)+\left(\rho_{P}^{\max }-\rho_{P}^{\min }\right) \mathbb{S}(P) \\
& +(1-\delta)\left(\mathbb{S} \sum_{u \in V_{G}} \rho(u) d(T, u, S)+\mathbb{P} \sum_{u \in V_{G}} \sigma(u) d(T, u, S)\right),
\end{aligned}
$$

onde $S=\bigcup_{P \in Y} P$ é o $\delta$ - $\sigma \rho$-separador minimal de $T$ associado a $Y$.

Demonstração. Como toda aresta é um caminho com dois vértices, se expressamos o custo de comunicação de $T$ como a soma do total de requerimento que passa por cada aresta em $T$ multiplicado 
pelo comprimento da aresta:

$$
C(T)=\sum_{e \in E_{T}}\left(\sigma\left(V\left(T, e, f_{e}\right)\right) \rho\left(V\left(T, e, l_{e}\right)\right)+\rho\left(V\left(T, e, f_{e}\right)\right) \sigma\left(V\left(T, e, l_{e}\right)\right)\right) \omega(e) .
$$

É possível dividir essa expressão na soma sobre as arestas em $S$ e aquelas fora de $S$ :

$$
\begin{aligned}
C(T)= & \sum_{e \in E_{S}}\left(\sigma\left(V\left(T, e, f_{e}\right)\right) \rho\left(V\left(T, e, l_{e}\right)\right)+\rho\left(V\left(T, e, f_{e}\right)\right) \sigma\left(V\left(T, e, l_{e}\right)\right)\right) \omega(e) \\
& +\sum_{e \in E_{T \backslash S}}\left(\sigma\left(V\left(T, e, f_{e}\right)\right) \rho\left(V\left(T, e, l_{e}\right)\right)+\rho\left(V\left(T, e, f_{e}\right)\right) \sigma\left(V\left(T, e, l_{e}\right)\right)\right) \omega(e) .
\end{aligned}
$$

Como $S$ é um $\delta$ - $\sigma \rho$-separador para toda aresta $e$ fora de $S$, o extremo mais distante de $S$, sem perda de generalidade $l_{e}$, satisfaz:

$$
\sigma\left(V\left(T, e, l_{e}\right)\right) \leq \delta \mathbb{S} \text { e } \rho\left(V\left(T, e, l_{e}\right)\right) \leq \delta \mathbb{P} .
$$

Isto implica que $f_{e}$ satisfaz:

$$
\sigma\left(V\left(T, e, f_{e}\right)\right) \geq(1-\delta) \mathbb{S} \text { e } \rho\left(V\left(T, e, f_{e}\right)\right) \geq(1-\delta) \mathbb{P}
$$

Logo:

$$
\begin{aligned}
& C(T) \geq \sum_{e \in E_{S}}\left(\sigma\left(V\left(T, e, f_{e}\right)\right) \rho\left(V\left(T, e, l_{e}\right)\right)+\rho\left(V\left(T, e, f_{e}\right)\right) \sigma\left(V\left(T, e, l_{e}\right)\right)\right) \omega(e) \\
& +\sum_{e \in E_{T \backslash S}}\left((\mathbf{1}-\boldsymbol{\delta}) \mathbb{S} \rho\left(V\left(T, e, l_{e}\right)\right)+(\mathbf{1}-\boldsymbol{\delta}) \mathbb{P} \sigma\left(V\left(T, e, l_{e}\right)\right)\right) \omega(e) \\
& \geq \sum_{e \in E_{S}}\left(\sigma\left(V\left(T, e, f_{e}\right)\right) \rho\left(V\left(T, e, l_{e}\right)\right)+\rho\left(V\left(T, e, f_{e}\right)\right) \sigma\left(V\left(T, e, l_{e}\right)\right)\right) \omega(e) \\
& +(\mathbf{1}-\boldsymbol{\delta}) \sum_{e \in E_{T \backslash S}}\left(\mathbb{S} \rho\left(V\left(T, e, l_{e}\right)\right)+\mathbb{P} \sigma\left(V\left(T, e, l_{e}\right)\right)\right) \omega(e) \\
& \geq \sum_{e \in E_{S}}\left(\sigma\left(V\left(T, e, f_{e}\right)\right) \rho\left(V\left(T, e, l_{e}\right)\right)+\rho\left(V\left(T, e, f_{e}\right)\right) \sigma\left(V\left(T, e, l_{e}\right)\right)\right) \omega(e) \\
& +(1-\delta)\left(\mathbb{S} \sum_{e \in E_{T \backslash S}} \rho\left(V\left(T, e, l_{e}\right)\right) \omega(e)+\mathbb{P} \sum_{e \in E_{T \backslash S}} \sigma\left(V\left(T, e, l_{e}\right)\right) \omega(e)\right) .
\end{aligned}
$$

Como $\sum_{e \in E_{T \backslash S}} \rho\left(V\left(T, e, l_{e}\right)\right) \omega(e)=\sum_{u \in V_{G \backslash S}} \rho(u) d(T, u, S)$ e $\sum_{e \in E_{T \backslash S}} \sigma\left(V\left(T, e, l_{e}\right)\right) \omega(e)=$ $\sum_{u \in V_{G \backslash S}} \sigma(u) d(T, u, S)$ :

$$
\begin{aligned}
C(T) \geq & \sum_{e \in E_{S}}\left(\sigma\left(V\left(T, e, f_{e}\right)\right) \rho\left(V\left(T, e, l_{e}\right)\right)+\rho\left(V\left(T, e, f_{e}\right)\right) \sigma\left(V\left(T, e, l_{e}\right)\right)\right) \omega(e) \\
& +(1-\delta)\left(\mathbb{S} \sum_{\boldsymbol{u} \in \boldsymbol{V}_{\boldsymbol{G} \backslash S}} \boldsymbol{\rho}(\boldsymbol{u}) \boldsymbol{d}(\boldsymbol{T}, \boldsymbol{u}, \boldsymbol{S})+\mathbb{P} \sum_{\boldsymbol{u} \in \boldsymbol{V}_{\boldsymbol{G} \backslash S}} \boldsymbol{\sigma}(\boldsymbol{u}) \boldsymbol{d}(\boldsymbol{T}, \boldsymbol{u}, \boldsymbol{S})\right) .
\end{aligned}
$$

A distância de todo vértice de $S$ para o próprio $S$ é zero, isto é $d(T, u, S)=0$ para qualquer 
$u \in V_{S}, \operatorname{logo}:$

$$
\begin{aligned}
C(T) \geq & \sum_{e \in E_{S}}\left(\sigma\left(V\left(T, e, f_{e}\right)\right) \rho\left(V\left(T, e, l_{e}\right)\right)+\rho\left(V\left(T, e, f_{e}\right)\right) \sigma\left(V\left(T, e, l_{e}\right)\right)\right) \omega(e) \\
& +(1-\delta)\left(\mathbb{S} \sum_{\boldsymbol{u} \in \boldsymbol{V}_{\boldsymbol{G}}} \rho(u) d(T, u, S)+\mathbb{P} \sum_{\boldsymbol{u} \in \boldsymbol{V}_{\boldsymbol{G}}} \sigma(u) d(T, u, S)\right) .
\end{aligned}
$$

Como todo par de caminhos em $Y$ é internamente disjunto, temos que cada aresta de $S$ está em exatamente um caminho de $Y$, o que implica:

$$
\begin{aligned}
C(T) \geq & \sum_{P \in Y} \sum_{e \in P}\left(\sigma\left(V\left(T, e, f_{e}\right)\right) \rho\left(V\left(T, e, l_{e}\right)\right)+\rho\left(V\left(T, e, f_{e}\right)\right) \sigma\left(V\left(T, e, l_{e}\right)\right)\right) \omega(e) \\
& +(1-\delta)\left(\mathbb{S} \sum_{\boldsymbol{u} \in \boldsymbol{V}_{\boldsymbol{G}}} \rho(u) d(T, u, S)+\mathbb{P} \sum_{\boldsymbol{u} \in \boldsymbol{V}_{\boldsymbol{G}}} \sigma(u) d(T, u, S)\right)
\end{aligned}
$$

Se $P=v_{1} \ldots v_{h}$ é um caminho em $Y$, temos:

$$
\begin{aligned}
& \sum_{e \in P}\left(\sigma\left(V\left(T, e, f_{e}\right)\right) \rho\left(V\left(T, e, l_{e}\right)\right)+\rho\left(V\left(T, e, f_{e}\right)\right) \sigma\left(V\left(T, e, l_{e}\right)\right)\right) \omega(e) \\
= & \sum_{i=1}^{h-1}\left(\sigma\left(V\left(T,\left(v_{i}, v_{i+1}\right), v_{i}\right)\right) \rho\left(V\left(T,\left(v_{i}, v_{i+1}\right), v_{i+1}\right)\right)\right) \omega\left(\left(v_{i}, v_{i+1}\right)\right) \\
& +\sum_{i=1}^{h-1}\left(\rho\left(V\left(T,\left(v_{i}, v_{i+1}\right), v_{i}\right)\right) \sigma\left(V\left(T,\left(v_{i}, v_{i+1}\right), v_{i+1}\right)\right)\right) \omega\left(\left(v_{i}, v_{i+1}\right)\right) .
\end{aligned}
$$

Para $i \in\{1, \ldots, h-1\}$ :

$$
\begin{aligned}
\sigma\left(V\left(T,\left(v_{i}, v_{i+1}\right), v_{i}\right)\right) & =\sigma_{P}^{f}+\sum_{k=2}^{i} \sigma\left(V\left(T, P, v_{k}\right)\right), \\
\sigma\left(V\left(T,\left(v_{i}, v_{i+1}\right), v_{i+1}\right)\right) & =\sigma_{P}^{l}+\sum_{k=i+1}^{h-1} \sigma\left(V\left(T, P, v_{k}\right)\right), \\
\rho\left(V\left(T,\left(v_{i}, v_{i+1}\right), v_{i}\right)\right) & =\rho_{P}^{f}+\sum_{k=2}^{i} \rho\left(V\left(T, P, v_{k}\right)\right), \\
\rho\left(V\left(T,\left(v_{i}, v_{i+1}\right), v_{i+1}\right)\right) & =\rho_{P}^{l}+\sum_{k=i+1}^{h-1} \rho\left(V\left(T, P, v_{k}\right)\right) .
\end{aligned}
$$

Logo:

$$
\begin{aligned}
& \sum_{e \in P}\left(\sigma\left(V\left(T, e, f_{e}\right)\right) \rho\left(V\left(T, e, l_{e}\right)\right)+\rho\left(V\left(T, e, f_{e}\right)\right) \sigma\left(V\left(T, e, l_{e}\right)\right)\right) \omega(e) \\
= & \sum_{i=1}^{h-1}\left(\left(\boldsymbol{\sigma}_{\boldsymbol{P}}^{f}+\sum_{\boldsymbol{k}=\mathbf{2}}^{\boldsymbol{i}} \boldsymbol{\sigma}\left(\boldsymbol{V}\left(\boldsymbol{T}, \boldsymbol{P}, \boldsymbol{v}_{\boldsymbol{k}}\right)\right)\right)\left(\boldsymbol{\rho}_{\boldsymbol{P}}^{\boldsymbol{l}}+\sum_{\boldsymbol{k}=\boldsymbol{i + 1}}^{\boldsymbol{h}-\boldsymbol{1}} \boldsymbol{\rho}\left(\boldsymbol{V}\left(\boldsymbol{T}, \boldsymbol{P}, \boldsymbol{v}_{\boldsymbol{k}}\right)\right)\right)\right) \omega\left(\left(v_{i}, v_{i+1}\right)\right) \\
& +\sum_{i=1}^{h-1}\left(\left(\boldsymbol{\rho}_{\boldsymbol{P}}^{\boldsymbol{f}}+\sum_{\boldsymbol{k}=\mathbf{2}}^{\boldsymbol{i}} \boldsymbol{\rho}\left(\boldsymbol{V}\left(\boldsymbol{T}, \boldsymbol{P}, \boldsymbol{v}_{\boldsymbol{k}}\right)\right)\right)\left(\boldsymbol{\sigma}_{\boldsymbol{P}}^{\boldsymbol{l}}+\sum_{\boldsymbol{k}=\boldsymbol{i}+\boldsymbol{1}}^{\boldsymbol{h}-\mathbf{1}} \boldsymbol{\sigma}\left(\boldsymbol{V}\left(\boldsymbol{T}, \boldsymbol{P}, \boldsymbol{v}_{\boldsymbol{k}}\right)\right)\right)\right) \omega\left(\left(v_{i}, v_{i+1}\right)\right)
\end{aligned}
$$




$$
\begin{aligned}
\geq & \left(\sigma_{P}^{f} \rho_{P}^{l}+\rho_{P}^{f} \sigma_{P}^{l}\right) \sum_{i=1}^{h-1} \omega\left(\left(v_{i}, v_{i+1}\right)\right) \\
& +\sigma_{P}^{f} \sum_{i=1}^{h-1} \sum_{k=i+1}^{h-1} \rho\left(V\left(T, P, v_{k}\right)\right) \omega\left(\left(v_{i}, v_{i+1}\right)\right)+\rho_{P}^{l} \sum_{i=1}^{h-1} \sum_{k=2}^{i} \sigma\left(V\left(T, P, v_{k}\right)\right) \omega\left(\left(v_{i}, v_{i+1}\right)\right) \\
& +\rho_{P}^{f} \sum_{i=1}^{h-1} \sum_{k=i+1}^{h-1} \sigma\left(V\left(T, P, v_{k}\right)\right) \omega\left(\left(v_{i}, v_{i+1}\right)\right)+\sigma_{P}^{l} \sum_{i=1}^{h-1} \sum_{k=2}^{i} \rho\left(V\left(T, P, v_{k}\right)\right) \omega\left(\left(v_{i}, v_{i+1}\right)\right) \\
= & \left(\sigma_{P}^{f} \rho_{P}^{l}+\rho_{P}^{f} \sigma_{P}^{l}\right) \omega(P)+\sigma_{P}^{f} \mathbb{P}^{f}(P)+\rho_{P}^{l} \mathbb{S}^{l}(P)+\rho_{P}^{f} \mathbb{S}^{f}(P)+\sigma_{P}^{l} \mathbb{P}^{l}(P) .
\end{aligned}
$$

Como $\left\{\sigma_{P}^{\max }, \sigma_{P}^{\min }\right\}=\left\{\sigma_{P}^{f}, \sigma_{P}^{f}\right\},\left\{\rho_{P}^{\max }, \rho_{P}^{\min }\right\}=\left\{\rho_{P}^{f}, \rho_{P}^{f}\right\}, \sigma_{P}^{m} \omega(P)=\mathbb{S}^{f}(P)+\mathbb{S}^{l}(P) \mathrm{e}$ $\rho_{P}^{m} \omega(P)=\mathbb{P}^{f}(P)+\mathbb{P}^{l}(P)$, temos:

$$
\begin{aligned}
& \sum_{e \in P}\left(\sigma\left(V\left(T, e, f_{e}\right)\right) \rho\left(V\left(T, e, l_{e}\right)\right)+\rho\left(V\left(T, e, f_{e}\right)\right) \sigma\left(V\left(T, e, l_{e}\right)\right)\right) \omega(e) \\
& \geq\left(\sigma_{P}^{f} \rho_{P}^{l}+\rho_{P}^{f} \sigma_{P}^{l}\right) \omega(P) \\
& +\sigma_{P}^{\max } \mathbb{P}(P)+\rho_{P}^{\max _{S}} \mathbb{S}(P)+\rho_{P}^{\min }\left(\sigma_{P}^{m}-\mathbb{S}(P)\right)+\sigma_{P}^{\min }\left(\rho_{P}^{m}-\mathbb{P}(P)\right) \\
& =\left(\sigma_{P}^{f} \rho_{P}^{l}+\rho_{P}^{f} \sigma_{P}^{l}+\boldsymbol{\sigma}_{\boldsymbol{P}}^{m} \boldsymbol{\rho}_{\boldsymbol{P}}^{\min }+\boldsymbol{\rho}_{\boldsymbol{P}}^{m} \boldsymbol{\sigma}_{\boldsymbol{P}}^{\min }\right) \omega(P) \\
& +\left(\rho_{P}^{\max }-\rho_{P}^{\min }\right) \mathbb{S}(P)+\left(\sigma_{P}^{\max }-\sigma_{P}^{\min }\right) \mathbb{P}(P) .
\end{aligned}
$$

O que nos permite concluir:

$$
\begin{aligned}
C(T) \geq & \sum_{P \in Y}\left(\rho_{P}^{l} \sigma_{P}^{f}+\rho_{P}^{f} \sigma_{P}^{l}+\rho_{P}^{\min } \sigma_{P}^{m}+\sigma_{P}^{\min } \rho_{P}^{m}\right) \omega(P) \\
& +\sum_{P \in Y}\left(\sigma_{P}^{\max }-\sigma_{P}^{\min }\right) \mathbb{P}(P)+\left(\rho_{P}^{\max }-\rho_{P}^{\min }\right) \mathbb{S}(P) \\
& +(1-\delta)\left(\mathbb{S} \sum_{u \in V_{G}} \rho(u) d(T, u, S)+\mathbb{P} \sum_{u \in V_{G}} \sigma(u) d(T, u, S)\right) .
\end{aligned}
$$

\subsection{Existência do spine limitado}

O lema que segue enuncia a existência para qualquer árvore geradora $T$ de $G$, de um $\delta$ - $\sigma \rho$ spine $Y$ de $T$ com o número de vértices que são extremos de caminho de $Y,|\operatorname{ext}(Y)|$, limitado superiormente por uma função de $\delta$.

Lema 5.14. Dados $0<\delta \leq \frac{1}{2}$ e uma árvore geradora $T$ de $G$, existe um $\delta$ - $\sigma \rho$-spine $Y$ de $T$ que satisfaz $|\operatorname{ext}(Y)| \leq 3\left(\left\lceil\frac{6}{\delta}\right\rceil^{2}-11\left\lceil\frac{6}{\delta}\right\rceil+1\right)$.

Demonstração. Na próxima seção demonstramos a proposição 5.16, que garante a existência de dois conjuntos $Y_{\rho}^{\prime}$ e $Y_{\sigma}^{\prime}$, cada um de $\delta$ - $\sigma \rho$-caminhos internamente disjuntos dois a dois, tais que $\cup_{P \in Y_{\rho}^{\prime}} P=S_{\rho}$ é um $\delta$ - $\rho$-separador minimal de $T, \cup_{P \in Y_{\sigma}^{\prime}} P=S_{\sigma}$ é um $\delta$ - $\sigma$-separador minimal de $T$, e:

$$
\left|\operatorname{ext}\left(Y_{\rho}^{\prime}\right)\right|,\left|\operatorname{ext}\left(Y_{\sigma}^{\prime}\right)\right| \leq\left\lceil\frac{6}{\delta}\right\rceil^{2}-11\left\lceil\frac{6}{\delta}\right\rceil+1
$$


A partir destes dois conjuntos de $\delta$ - $\sigma \rho$-caminhos é possível construir um novo conjunto que seja um $\delta$ - $\sigma \rho$-separador de $T$.

Para isto, primeiro definiremos dois novos conjuntos $Y_{\rho}$ e $Y_{\sigma}$ de $\delta$ - $\sigma \rho$-caminhos internamente disjuntos dois a dois, onde nenhum caminho de $Y_{\rho}$ tem vértices internos em $\operatorname{ext}\left(Y_{\sigma}\right)$ e vice-versa.

Para cada $P \in Y_{\rho}^{\prime}$ se $P$ contém vértices internos que estão em $\operatorname{ext}\left(Y_{\sigma}^{\prime}\right)$, então dividimos $P$ nesses vértices para criar novos $\delta$ - $\sigma \rho$-caminhos internamente disjuntos dois a dois, colocando esses caminhos em $Y_{\rho}$, caso contrário $P$ é adicionado a $Y_{\rho}$. De forma simétrica definimos $Y_{\sigma}$ a partir de $Y_{\sigma}^{\prime}$ e $\operatorname{ext}\left(Y_{\rho}^{\prime}\right)$.

Observe que nenhum caminho em $Y_{\sigma} \cup Y_{\rho}$ tem vértices internos que pertencem a $\operatorname{ext}\left(Y_{\sigma}\right) \cup \operatorname{ext}\left(Y_{\rho}\right)$. Além disto, $Y_{\sigma}$ e $Y_{\rho}$ são conjuntos de $\delta$ - $\sigma \rho$-caminhos disjuntos dois a dois tais que $\cup_{P \in Y_{\rho}} P=S_{\rho}$, $\cup_{P \in Y_{\sigma}} P=S_{\sigma}$ e:

$$
\left|\operatorname{ext}\left(Y_{\rho} \cup Y_{\sigma}\right)\right| \leq 2\left(\left\lceil\frac{6}{\delta}\right\rceil^{2}-11\left\lceil\frac{6}{\delta}\right\rceil+1\right) .
$$

Como $S_{\sigma} \cup S_{\rho}$ não tem ciclos, pois é a união de sub-árvores de $T$ (que é também uma árvore), temos que cada caminho de $Y_{\rho}$ tem interseção de vértices internos com no máximo um caminho em $Y_{\sigma}$, e o mesmo acontece para os caminhos de $Y_{\sigma}$.

Assim, analisamos três possibilidades:

- A primeira possibilidade, ilustrada pela Figura 5.4, é o caso em que dois caminhos $P_{\sigma} \in Y_{\sigma}$ e $P_{\rho} \in Y_{\rho}$ têm vértices internos em comum e não têm extremos em comum. Neste caso, nenhum outro caminho de $Y_{\sigma}$ pode ter interseção com um caminho de $Y_{\rho}$, pois caso contrário teríamos um ciclo e todos estes são sub-caminhos de uma mesma árvore $T$. Então, removendo de $P_{\sigma}$ os vértices internos da interseção, adicionamos no máximo dois vértices que são extremos de caminhos (aqueles que são os extremos da interseção). Logo, $Y^{\prime}=\left(Y_{\sigma} \backslash P_{\sigma}\right) \cup Y_{\rho} \cup\left(P_{\sigma} \backslash\left(P_{\sigma} \cap P_{\rho}\right)\right)$ é um conjunto de $\delta$ - $\sigma \rho$-caminhos internamente disjuntos dois a dois que satisfaz $\cup_{P \in Y^{\prime}} P=S^{\prime}$ é um $\delta$ - $\sigma \rho$-separador de $T$ e:

$$
\left|\operatorname{ext}\left(Y^{\prime}\right)\right| \leq 2\left(\left\lceil\frac{6}{\delta}\right\rceil^{2}-11\left\lceil\frac{6}{\delta}\right\rceil+1\right)+2 .
$$

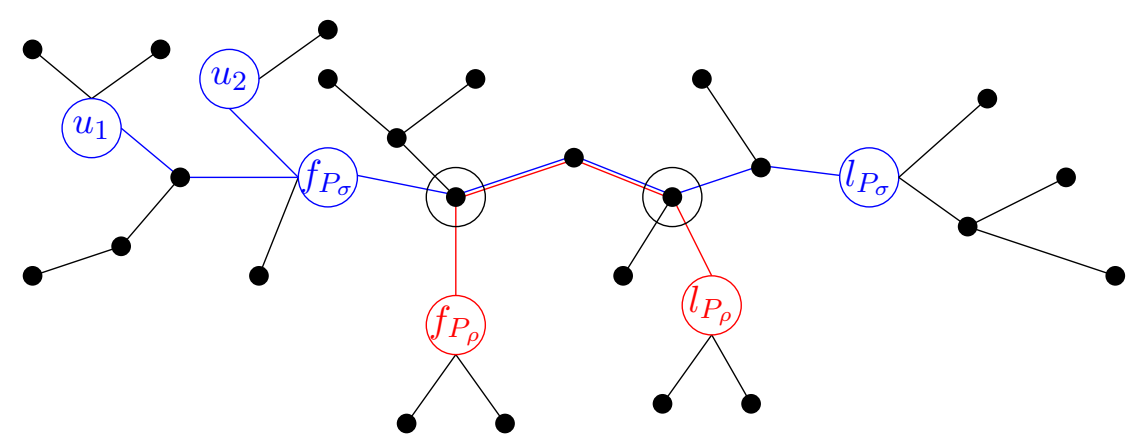

Figura 5.4: Observe que no exemplo, $Y_{\sigma}$ é o conjunto de três caminhos contendo os caminhos entre $u_{1} e$ $f_{P_{\sigma}}, u_{2}$ e $f_{P_{\sigma}}$, e o caminho $P_{\sigma}$ entre $f_{P_{\sigma}}$ e $l_{P_{\sigma}}$. $Y_{\rho}$ é o conjunto de um único caminho, sendo este $P_{\rho}$ entre os vértices $f_{P_{\rho}}$ e $l_{P_{\rho}}$. $Y^{\prime}$ resulta no conjunto de 7 caminhos, que contém os caminhos de $Y_{\sigma}$ entre $u_{1}$ e $f_{P_{\sigma}}$, e $u_{2}$ e $f_{P_{\sigma}}$, além de conter os 5 caminhos que se obtém quando quebramos $P_{\sigma}$ e $P_{\rho}$ nos vértices que são extremos da interseção entre eles (em evidência com uma circunferência ao redor).

- A segunda possibilidade, ilustrada pela Figura 5.5, é o caso em ainda há interseção entre 
caminhos de $Y_{\sigma}$ com caminhos de $Y_{\rho}$, porém qualquer interseção não vazia entre um caminho de $Y_{\sigma}$ e um de $Y_{\rho}$ contém ao menos um vértice extremo de pelo menos um dos caminhos. Neste caso, eliminamos de cada caminho em $Y_{\sigma}$ os vértices internos da interseção com cada caminho em $Y_{\rho}$ (onde, um caminho de $Y_{\sigma}$ no máximo tem interseção interna com um caminho em $Y_{\rho}$ ). Neste caso o número de vértices extremos de novos extremos de caminhos será no máximo $\left|Y_{\sigma}^{\prime}\right|$ e definimos o conjunto $Y^{\prime}$ como a união de $Y_{\rho}$ com o $Y_{\sigma}$ modificado, o que é um conjunto de $\delta$ - $\sigma \rho$-caminhos internamente disjuntos dois a dois que satisfaz $\cup_{P \in Y^{\prime}} P=S^{\prime}$ é um $\delta$ - $\sigma \rho$-separador de $T$ e:

$$
\left|\operatorname{ext}\left(Y^{\prime}\right)\right| \leq 3\left(\left\lceil\frac{6}{\delta}\right\rceil^{2}-11\left\lceil\frac{6}{\delta}\right\rceil+1\right)
$$

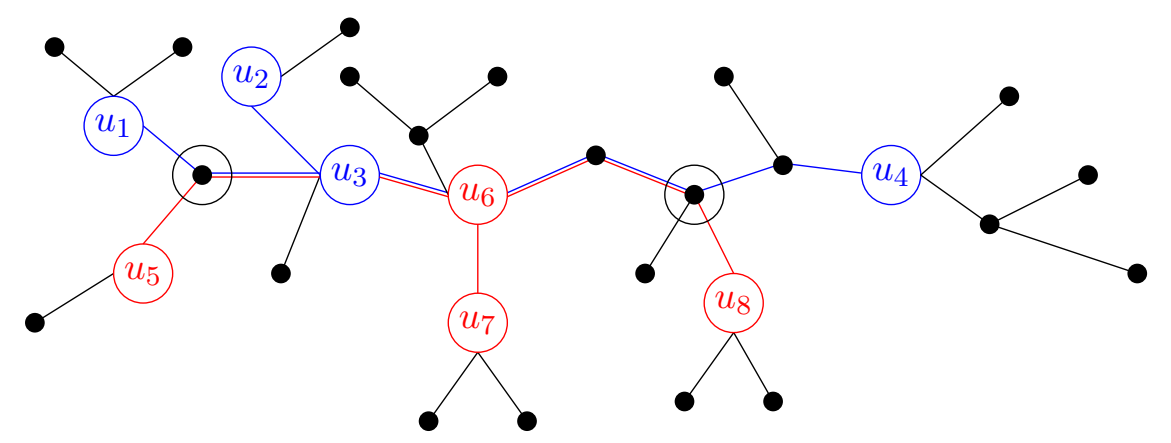

Figura 5.5: No exemplo, $Y_{\sigma}$ é o conjunto de três caminhos contendo os caminhos entre $u_{1}$ e $u_{3}, u_{2}$ e $u_{3}$, e $u_{3}$ e $u_{4}$. $Y_{\rho}$ é o conjunto de três caminhos contendo os caminhos entre $u_{5}$ e $u_{6}, u_{6}$ e $u_{7}$, e $u_{6}$ e $u_{8}$. Quando consideramos dois novos extremos de caminhos (os vértices em evidência com uma circunferência ao redor), temos que $Y^{\prime}$ resulta no conjunto de 9 caminhos definidos entre os extremos dos caminhos em $Y_{\sigma}$, $Y_{\rho}$ e os dois novos extremos considerados.

- A última possibilidade é que nenhum caminho de $Y_{\sigma}$ tem interseção com caminhos de $Y_{\rho}$. Neste caso, como $\cup_{P \in Y_{\rho}}=S_{\rho}$ e $\cup_{P \in Y_{\sigma}}=S_{\sigma}$ são sub-árvores de $T$, temos que $S_{\sigma}$ pertence a alguma componente de $T \backslash S_{\rho}$; e como $S_{\rho}$ é um $\delta$ - $\rho$-separador de $T$, toda componente $B$ em $T \backslash S_{\rho}$ satisfaz $\rho(B) \delta \mathbb{P}$, logo o caminho $P$ de $T$ que conecta $S_{\rho}$ com $S_{\sigma}$ satisfaz $\rho_{P}^{m}<\delta \mathbb{P}$. Analogamente, $P$ também satisfaz $\sigma_{P}^{m}<\delta \mathbb{S}$. Portanto, $P$ pode ser dividido em 6 sub-caminhos $P_{1}^{\rho}, \cdots, P_{6}^{\rho}$ que satisfazem $\rho_{P_{i}^{\rho}}^{m} \leq \frac{\delta R_{s}}{6}$, e também pode ser divido em outros 6 sub-caminhos $P_{1}^{\sigma}, \cdots, P_{6}^{\sigma}$ que satisfazem $\sigma_{P_{i}^{\sigma}}^{m} \leq \frac{\delta R_{r}}{6}(i \in\{1, \cdots, 6\})$. Como cada uma destas divisões usa 5 vértices internos de $P$, no pior caso, usando 10 vértices internos de $P$ conseguimos uma divisão de $P$ em no máximo $11 \delta$ - $\sigma \rho$-caminhos, cuja união com $Y_{\sigma}$ e $Y_{\rho}$ resulta em um conjunto $Y^{\prime}$ de $\delta$ - $\sigma \rho$-caminhos internamente disjuntos dois a dois tais que $\cup_{P \in Y^{\prime}} P=S^{\prime}$ é um $\delta$ - $\sigma \rho$-separador de $T$ e:

$$
\left|\operatorname{ext}\left(Y^{\prime}\right)\right| \leq 2\left(\left\lceil\frac{6}{\delta}\right\rceil^{2}-11\left\lceil\frac{6}{\delta}\right\rceil+1\right)+10
$$

Observe que, para $0<\delta \leq \frac{1}{2}$, se satisfaz:

$$
\left(\left\lceil\frac{6}{\delta}\right\rceil^{2}-11\left\lceil\frac{6}{\delta}\right\rceil+1\right) \geq 12^{2}-11(12)+1=13>10,
$$

logo em qualquer caso o conjunto $Y^{\prime}$ de $\delta$ - $\sigma \rho$-caminhos internamente disjuntos dois a dois satisfaz 
$\cup_{P \in Y^{\prime}} P=S^{\prime}$ é um $\delta$ - $\sigma \rho$-separador de $T$ e:

$$
\left|\operatorname{ext}\left(Y^{\prime}\right)\right| \leq 3\left(\left\lceil\frac{6}{\delta}\right\rceil^{2}-11\left\lceil\frac{6}{\delta}\right\rceil+1\right) .
$$

Se $S^{\prime}$ é um $\delta$ - $\sigma \rho$-separador minimal de $T$, então $Y=Y^{\prime}$ é um $\delta$ - $\sigma \rho$-spine. Caso contrário, existe um $\delta$ - $\sigma \rho$-separador minimal $S \subset S^{\prime}$ e se eliminamos de cada caminho em $Y^{\prime}$ os elementos que não pertencem a $S$ obtemos um $\delta$ - $\sigma \rho$-spine $Y$ de $T$ que satisfaz:

$$
|\operatorname{ext}(Y)| \leq 3\left(\left\lceil\frac{6}{\delta}\right\rceil^{2}-11\left\lceil\frac{6}{\delta}\right\rceil+1\right)
$$

\subsubsection{Existência de spines de requerimento de envio e de recepção}

Primeiro mostramos um resultado obtido em [WLB ${ }^{+}$98], [WCT00a] e [WCT00b]:

Fato 5.15. Dados $0<\delta \leq \frac{1}{2}$ e um $\delta$ - $\rho$-separador ( $\delta$ - $\sigma$-separador) minimal $S$ de uma árvore geradora $T$ de $G$, se u é uma folha de $S$, então $\rho(V(T, S, u))>\delta \mathbb{P}(\sigma(V(T, S, u))>\delta \mathbb{S})$.

Demonstração. Se $n(S)=1$, então $u$ é o único elemento em $S$ e trivialmente temos: $\rho(V(T, S, u))=$ $\mathbb{P}>\frac{1}{2} \mathbb{P} \geq \delta \mathbb{P}\left(\sigma(V(T, S, u))=\mathbb{S}>\frac{1}{2} \mathbb{S} \geq \delta \mathbb{S}\right)$. Caso contrário, $n(S)>1$, suponha que $\rho(V(T, S, u)) \leq$ $\delta \mathbb{P}(\sigma(V(T, S, u)) \leq \delta \mathbb{S})$, temos que $S \backslash u$ ainda é um $\delta$ - $\rho$-separador ( $\delta$ - $\sigma$-separador) de $T$, o que implica que $S$ não é minimal e isto é uma contradição.

Agora provamos o resultado usado no lema 5.14:

Proposição 5.16. Dados $0<\delta \leq \frac{1}{2}$ e um $\delta$ - $\rho$-separador ( $\delta$ - $\sigma$-separador) minimal $S$ de uma árvore geradora $T$ de $G$, existe um conjunto $Y$ de $\delta$ - $\sigma \rho$-caminhos de $T$ internamente disjuntos dois a dois que satisfaz $|\operatorname{ext}(Y)| \leq\left\lceil\frac{6}{\delta}\right\rceil^{2}-11\left\lceil\frac{6}{\delta}\right\rceil+1 e \cup_{P \in Y} P=S$.

Demonstração. Sejam $U_{1}$ o conjunto das folhas de $S, U_{2}$ o conjunto dos vértices de grau dois em $S$ e $U_{>2}$ o conjunto dos vértices com grau maior que dois em $S$. Como $S$ é uma árvore $\left|U_{1}\right| \geq\left|U_{>2}\right|+2$, temos que $U=U_{1} \cup U_{>2}$ satisfaz $|U| \leq 2\left|U_{1}\right|+2$.

Dizemos que $u, v \in U$, são vizinhos em $U$ se para qualquer $w \in U \backslash\{u, v\}, w$ não pertence ao caminho entre $u$ e $v$ em $T$. Logo definimos o conjunto $Y_{1}$ como:

$$
Y_{1}=\{P \mid P \text { é um caminho entre } u \text { e } v \text { em } T \text {, onde } u, v \in U \text { são vizinhos em } U\}
$$

Também classificamos o caminho $P \in Y_{1}$ como $\rho$-pesado ( $\sigma$-pesado) se $\rho_{P}^{m}>\delta \frac{R}{6}\left(\sigma_{P}^{m}>\delta \frac{n}{6}\right)$.

Observe que é possível dividir um caminho $\rho$-pesado ( $\sigma$-pesado) $P$ em sub-caminhos não $\rho$ pesados $\left(\sigma\right.$-pesados) usando $\left\lceil\frac{6 \rho_{P}^{m}}{\delta \mathbb{P}}\right\rceil-1\left(\left\lceil\frac{6 \sigma_{P}^{m}}{\delta \mathbb{S}}\right\rceil-1\right)$ vértices para a divisão (isto é, vértices internos de $P$ que serão extremos dos sub-caminhos gerados).

Como $S$ é um $\delta$ - $\rho$-separador ( $\delta$ - $\sigma$-separador) minimal de $T$, o fato 5.15 garante que cada folha 
$u$ de $S$ satisfaz $\rho(V(T, S, u))>\delta \mathbb{P}\left(\sigma\left(V B\left(T, E_{S}, u\right)\right)>\delta \mathbb{S}\right)$. Logo:

$$
\sum_{P \in Y_{1}} \rho_{P}^{m}<\mathbb{P}\left(1-\delta\left|U_{1}\right|\right) \quad\left(\sum_{P \in Y_{1}} \sigma_{P}^{m}<\mathbb{S}\left(1-\delta\left|U_{1}\right|\right)\right) .
$$

Se denotamos por $U_{3}$ o conjunto de vértices usados para dividir todos os caminhos $\rho$-pesados $\left(\sigma\right.$-pesados) de $Y_{1}$ em caminhos não $\rho$-pesados ( $\sigma$-pesados), então:

$$
\left|U_{3}\right| \leq \sum_{P \in Y_{1}}\left(\left\lceil\frac{6 \rho_{P}^{m}}{\delta \mathbb{P}}\right\rceil-1\right)<\frac{6}{\delta \mathbb{P}} \mathbb{P}\left(1-\delta\left|U_{1}\right|\right) \leq\left\lceil\frac{6}{\delta}\right\rceil-6\left|U_{1}\right| .
$$

Se $Y_{2}$ representa o conjunto de caminhos obtidos ao dividir todos os caminhos $\rho$-pesados $(\sigma$ pesados) de $Y_{1}$ em caminhos não $\rho$-pesados ( $\sigma$-pesados), então temos:

$$
\left|\operatorname{ext}\left(Y_{2}\right)\right|=U+U_{3} \leq 2\left|U_{1}\right|-2+\left\lceil\frac{6}{\delta}\right\rceil-6\left|U_{1}\right| \leq\left\lceil\frac{6}{\delta}\right\rceil-10 .
$$

Agora dividimos cada caminho $\sigma$-pesado ( $\rho$-pesado) de $Y_{2}$ em caminhos não $\sigma$-pesados ( $\rho$ pesados), para obter um conjunto $Y$ de $\delta$ - $\sigma \rho$-caminhos. Logo, denotemos por $U_{4}$ o conjunto de vértices usados na divisão dos caminhos $\sigma$-pesados ( $\rho$-pesados) de $Y_{2}$ :

$$
\left|U_{4}\right| \leq \sum_{P \in Y_{2}}\left\lceil\frac{6 \sigma_{P}^{m}}{\delta n}\right\rceil-1 \leq \sum_{P \in Y_{2}}\left\lceil\frac{6}{\delta}\right\rceil-1 \leq\left|Y_{2}\right|\left(\left\lceil\frac{6}{\delta}\right\rceil-1\right) .
$$

Onde $\left|Y_{2}\right|=\operatorname{ext}\left(Y_{2}\right)-1$, portanto:

$$
|\operatorname{ext}(Y)|=\left|\operatorname{ext}\left(Y_{2}\right)\right|+\left|U_{4}\right| \leq\left\lceil\frac{6}{\delta}\right\rceil^{2}-11\left\lceil\frac{6}{\delta}\right\rceil+1 .
$$

Finalmente, observe que por construção todos caminhos em $Y$ conjunto são internamente disjuntos dois a dois.

\subsection{Teorema de aproximação para WSDOCT métrico}

Usando os lemas 5.9 e 5.14 , temos que para quaisquer $0<\delta \leq \frac{1}{2}$ e árvore geradora $T$ de $G$, existe um $\left(3\left(\left\lceil\frac{6}{\delta}\right\rceil^{2}-11\left\lceil\frac{6}{\delta}\right\rceil+1\right)\right)$-centro $\delta$-balanceado $X$ de $G$ tal que $C(X) \leq \frac{1}{1-\delta} C(T)$. Logo, para uma solução ótima do WSDOCT métrico $T^{*}$, existe um $\left(3\left(\left\lceil\frac{6}{\delta}\right\rceil^{2}-11\left\lceil\frac{6}{\delta}\right\rceil+1\right)\right)$-centro $\delta$-balanceado $X^{\prime}$ de $G$ tal que $C\left(X^{\prime}\right) \leq \frac{1}{1-\delta} C\left(T^{*}\right)$, e como um $\left(3\left(\left\lceil\frac{6}{\delta}\right\rceil^{2}-11\left\lceil\frac{6}{\delta}\right\rceil+1\right)\right)$-centro $\delta$-balanceado ótimo $X^{*}$ satisfaz $C\left(X^{*}\right) \leq C\left(X^{\prime}\right)$, concluímos o seguinte resultado:

Teorema 5.17. Dados $0<\delta \leq \frac{1}{2}$ e uma instância $I$ do WSDOCT métrico, um k-centro $\delta$ balanceado ótimo associado a $I$, com $k=\left(3\left(\left\lceil\frac{6}{\delta}\right\rceil^{2}-11\left\lceil\frac{6}{\delta}\right\rceil+1\right)\right)$, é uma $\left(\frac{1}{1-\delta}\right)$-aproximação de $I$.

No próximo capítulo usamos este teorema para mostrar que o WSDOCT métrico admite um PTAS. 


\section{Capítulo 6}

\section{PTAS para o WSDOCT métrico}

Neste capítulo mostramos a existência de um PTAS para o WSDOCT métrico. Para isto, assumimos que são dados $0<\delta \leq \frac{1}{2}$ e uma instância do WSDOCT métrico.

Tome $k=\left(3\left(\left\lceil\frac{6}{\delta}\right\rceil^{2}-11\left\lceil\frac{6}{\delta}\right\rceil+1\right)\right)$. Observe que o número de possíveis árvores que podem ser esqueleto de um $k$-centro é $O\left(k^{k} n^{k}\right)=O\left(n^{k}\right)$. Nas próximas seções, dada uma sub-árvore $\tau$ de $G$ com $k$ vértices, mostramos como encontrar em tempo $O\left(\left(n+\frac{n}{\delta^{2}}\right)^{2 k} \log ^{2}\left(n+\frac{n}{\delta^{2}}\right)\right)$, uma $(1+7 \delta)$-aproximação de um $k$-centro $\delta$-balanceado sobre $\tau$, o que pelo teorema 5.17 nos dá uma $\left(\frac{1+7 \delta}{1-\delta}\right)$-aproximação para WSDOCT.

Teorema 6.1. Existe um PTAS para o WSDOCT métrico, tal que uma $\left(1+\frac{8 \delta}{1-\delta}\right)$-aproximação pode ser encontrada em tempo $O\left(n^{k}\left(n+\frac{n}{\delta^{2}}\right)^{2 k} \log ^{2}\left(n+\frac{n}{\delta^{2}}\right)\right)$, onde $k=\left(3\left(\left\lceil\frac{6}{\delta}\right\rceil^{2}-11\left\lceil\frac{6}{\delta}\right\rceil+1\right)\right)$ $e<\delta \leq \frac{1}{2}$.

Assim, na Seção 6.1 mostramos como reduzir o problema de encontrar o $k$-centro $\delta$-balanceado ótimo sobre $\tau$ para o de encontrar o $k$-centro ótimo sobre $\tau$ em que os requerimentos de recepção nas folhas são inteiros. Na Seção 6.2, dado o problema de encontrar o $k$-centro ótimo sobre $\tau$ com requerimentos de recepção inteiros nas folhas, mostramos uma redução dele ao caso particular em que os requerimentos de recepção nas folhas são binários. Finalmente, na Seção 6.3, mostramos como encontrar o $k$-centro ótimo sobre $\tau$ onde os requerimentos de recepção nas folhas são binários.

\subsection{Razão de aproximação para um $k$-centro ótimo}

Dados $0<\delta \leq \frac{1}{2}$, uma sub-árvore $\tau$ de $G$ e $k=n(\tau)$, nesta seção mostramos como aproximar um $k$-centro $\delta$-balanceado ótimo $X$ sobre $\tau$ (se $X$ existe). Esta demonstração está inspirada em trabalhos prévios de Wu et al. [WCT00a, WCT00b].

A seguinte proposição dá uma limitante inferior para o requerimento que passa sobre qualquer aresta de $\tau$ em $X$.

Proposição 6.2. Dada uma aresta $e \in E_{\tau}$, o requerimento que passa por e em $X$ é no mínimo $\frac{\delta}{2} \mathbb{S P}$.

Demonstração. Se $X_{u}$ e $X_{v}$ são as sub-árvores de $X$ obtidas ao remover a aresta $e$, então o requerimento que passa por é igual a:

$$
\sigma\left(X_{u}\right) \rho\left(X_{v}\right)+\rho\left(X_{u}\right) \sigma\left(X_{v}\right)=\sigma\left(X_{u}\right)\left(\mathbb{P}-\rho\left(X_{u}\right)\right)+\rho\left(X_{u}\right)\left(\mathbb{S}-\sigma\left(X_{u}\right)\right)
$$


Como $X$ é um $k$-centro $\delta$-balanceado, temos que:

$$
\begin{array}{rll}
\sigma\left(X_{u}\right)>\delta \mathbb{S} & \text { ou } & \rho\left(X_{u}\right)>\delta \mathbb{P} \\
\mathbb{S}-\sigma\left(X_{u}\right)>\delta \mathbb{S} & \text { ou } & \mathbb{P}-\rho\left(X_{u}\right)>\delta \mathbb{P} .
\end{array}
$$

Analisaremos somente o caso em que $\sigma\left(X_{u}\right)>\delta \mathbb{S}$, pois o outro caso em que $\rho\left(X_{u}\right)>\delta \mathbb{P}$ é simétrico. Se $\mathbb{S}-\sigma\left(X_{u}\right) \leq \delta \mathbb{S}$, então $\mathbb{P}-\rho\left(X_{u}\right)>\delta \mathbb{P}$ e $\sigma\left(X_{u}\right) \geq \mathbb{S}-\delta \mathbb{S} \geq \frac{\mathbb{S}}{2}$, logo o requerimento que passa por e é no mínimo:

$$
\frac{\mathbb{S}}{2} \delta \mathbb{P}+\rho\left(X_{u}\right)\left(\mathbb{S}-\sigma\left(X_{u}\right)\right) \geq \frac{\delta}{2} \mathbb{S P} .
$$

Caso contrário, $\mathbb{S}-\sigma\left(X_{u}\right)>\delta \mathbb{S}$ e o requerimento que passa por e é no mínimo:

$$
\delta \mathbb{S}\left(\mathbb{P}-\rho\left(X_{u}\right)\right)+\rho\left(X_{u}\right)(\delta \mathbb{S})=\delta \mathbb{S P} \geq \frac{\delta}{2} \mathbb{S P} .
$$

Se denotamos por $V_{L}=V \backslash V_{\tau}=\left\{u_{1}, \cdots, u_{n-k}\right\}$, o conjunto de folhas que serão adicionadas a $\tau$ para construir uma solução, e consideramos um ordenamento sobre os elementos de $V_{L}$ tal que se $i<j$ então $\rho\left(u_{i}\right) \leq \rho\left(u_{j}\right)$ para todo $i, j \in\{1, \cdots, n-k\}$. Então, fazendo $m$ o valor máximo em $\{1, \cdots, n-k\}$, tal que $\sum_{i=1}^{m} \rho(u) \leq \delta^{2} \mathbb{P}$ e considerando $V_{L}^{0}=\left\{u_{1}, \cdots, u_{m}\right\}$; definimos uma nova função de requerimento de recepção $\rho_{1}$, tal que $\rho_{1}(u)=0$ se $u \in V_{L}^{0}$ e, caso contrário, $\rho_{1}(u)=\rho(u)$.

Agora, considerando o valor $\mu=1$ se $V_{L} \backslash V_{L}^{0}=\emptyset$ e, caso contrário, fazendo $\mu$ igual ao valor mínimo de $\rho_{1}$ no conjunto $V_{L} \backslash V_{L}^{0}$, definimos uma outra função de requerimento de recepção $\rho_{2}$ tal que $\rho_{2}(u)=\left\lfloor\frac{\rho_{1}(u)}{\delta \mu}\right\rfloor$ se $u \in V_{L} \backslash V_{L}^{0}$, caso contrário $\rho_{2}(u)=\frac{\rho_{1}(u)}{\delta \mu}$. Observe que qualquer $k$-centro $T$ com esqueleto $\tau$ satisfaz:

$$
\frac{1}{\delta \mu(1+\delta)} C\left(T, \sigma, \rho_{1}\right) \leq C\left(T, \sigma, \rho_{2}\right) \leq \frac{\rho_{1}}{\delta \mu} C\left(T, \sigma, \rho_{1}\right) .
$$

Onde, $C(T, \alpha, \beta)$ denota o custo de comunicação da árvore $T$ usando as funções de requerimento de envio $\alpha$ e de recepção $\beta$.

Se $X_{2}$ é um $k$-centro com esqueleto $\tau$, ótimo para o WSDOCT métrico usando a função de requerimento de recepção $\rho_{2}$, então:

$$
\frac{1}{\delta \mu(1+\delta)} C\left(X_{2}, \sigma, \rho_{1}\right) \leq C\left(X_{2}, \sigma, \rho_{2}\right) \leq C\left(X, \sigma, \rho_{2}\right) \leq \frac{1}{\delta \mu} C\left(X, \sigma, \rho_{1}\right) .
$$

Observe que para qualquer $k$-centro $T$ com esqueleto $\tau$ :

$$
C(T, \sigma, \rho)=C\left(T, \sigma, \rho_{1}\right)+\sum_{i=1}^{m} \rho\left(u_{i}\right) \sum_{v \in V} d\left(T, u_{i}, v\right) \sigma(v),
$$

como para cada $i \in\{1, \cdots, m\}, u_{i}$ é uma folha de $T$, denotando por $p_{T}\left(u_{i}\right)$ o vértice em $\tau$ adjacente em $T$ a $u_{i}$, temos que $d\left(T, u_{i}, v\right)=d\left(T, u_{i}, p_{T}\left(u_{i}\right)\right)+d\left(T, p_{T}\left(u_{i}\right), v\right)$ e:

$$
C(T, \sigma, \rho)=C\left(T, \sigma, \rho_{1}\right)
$$




$$
\begin{aligned}
& +\sum_{i=1}^{m} \rho\left(u_{i}\right) \sum_{v \in V_{L}^{0}}\left(d\left(T, u_{i}, p_{T}\left(u_{i}\right)\right)+d\left(T, p_{T}(v), v\right)\right) \sigma(v) \\
& +\sum_{i=1}^{m} \rho\left(u_{i}\right) \sum_{v \in V_{L}^{0}} d\left(T, p_{T}\left(u_{i}\right), p_{T}(v)\right) \sigma(v) \\
& +\sum_{i=1}^{m} \rho\left(u_{i}\right) \sum_{v \in V \backslash V_{L}^{0}}\left(d\left(T, u_{i}, p_{T}\left(u_{i}\right)\right)+d\left(T, p_{T}\left(u_{i}\right), v\right)\right) \sigma(v) .
\end{aligned}
$$

Denotando por $\omega(\tau)$ a soma de todos os comprimentos das arestas de $\tau$, como o grafo é métrico, temos que para cada $u \in V_{L}^{0}$ :

$$
d\left(T, u, p_{T}(u)\right) \leq d\left(X, u, p_{X}(u)\right)+d\left(\tau, p_{T}(u), p_{X}(u)\right) \leq d\left(X, u, p_{X}(u)\right)+\omega(\tau) .
$$

Logo:

$$
\begin{aligned}
C(T, \sigma, \rho) \leq & C\left(T, \sigma, \rho_{1}\right)+\sum_{i=1}^{m} 3 \rho\left(u_{i}\right) \mathbb{S} \omega(\tau) \\
& +\sum_{i=1}^{m} \rho\left(u_{i}\right) \sum_{v \in V_{L}^{0}}\left(d\left(X, u_{i}, p_{X}\left(u_{i}\right)\right)+d\left(X, p_{X}(v), v\right)\right) \sigma(v) \\
& +\sum_{i=1}^{m} \rho\left(u_{i}\right) \sum_{v \in V \backslash V_{L}^{0}}\left(d\left(X, u_{i}, p_{X}\left(u_{i}\right)\right)+d\left(X, p_{X}\left(u_{i}\right), v\right)\right) \sigma(v) .
\end{aligned}
$$

Lembre que $C\left(X_{2}, \sigma, \rho_{1}\right) \leq(1+\delta) C\left(X, \sigma, \rho_{1}\right)$, isto implica:

$$
\begin{aligned}
C\left(X_{2}, \sigma, \rho\right) & \leq(1+\delta) C(X, \sigma, \rho)+3 \mathbb{S} \omega(\tau) \sum_{i=1}^{m} \rho\left(u_{i}\right) \\
& \leq(1+\delta) C(X, \sigma, \rho)+3 \delta^{2} \mathbb{S P} \omega(\tau)
\end{aligned}
$$

Pela proposição 6.2 temos:

$$
C\left(X_{2}, \sigma, \rho\right) \leq(1+\delta) C(X, \sigma, \rho)+3 \delta(2 C(X, \sigma, \rho)) \leq(1+7 \delta) C(X, \sigma, \rho) .
$$

Nas próximas seções mostramos como obter $X_{2}$ em tempo $O\left(\left(n+\mathbb{P}_{\rho_{2}}\right)^{2 k} \log ^{2}\left(n+\mathbb{P}_{\rho_{2}}\right)\right)$. Onde $\mathbb{P}_{\beta}$ é a soma dos requerimentos de recepção de todos os vértices do grafo usando a função de requerimento $\beta$. Como $\delta^{2} \mathbb{P} \leq(m+1) \mu \leq n \mu$ e $\mathbb{P}_{\rho_{2}} \leq \frac{\mathbb{P}}{\mu}$, temos que $\mathbb{P}_{\rho_{2}} \leq \frac{n \mathbb{P}}{\delta^{2} \mathbb{P}}=\frac{n}{\delta^{2}}$.

Lema 6.3. Dados $0<\delta \leq \frac{1}{2}$ e uma sub-árvore $\tau$ de $G$ com $k$ vértices, se existe um $k$-centro $\delta$-balanceado de $G$ com esqueleto $\tau$, então existe uma $(1+7 \delta)$-aproximação para o $k$-centro $\delta$ balanceado com esqueleto $\tau$ ótimo para o WSDOCT métrico, que pode ser encontrada em tempo $O\left(\left(n+\frac{n}{\delta^{2}}\right)^{2 k} \log ^{2}\left(n+\frac{n}{\delta^{2}}\right)\right)$. 


\section{$6.2 k$-centro ótimo com requerimentos de recepção inteiros nas fo- lhas}

Nesta seção consideramos que é dada uma sub-árvore $\tau$ de $G$ com $k$ vértices e uma instância do WSDOCT métrico com os requerimentos de recepção inteiros, isto é $\rho(u) \in \mathbb{N}^{+}$para cada $u \in V \backslash V_{\tau}$; e mostramos como obter um $k$-centro com esqueleto $\tau$ ótimo para a instância do WSDOCT dada em tempo $O\left((n+\mathbb{P})^{2 k} \log ^{2}(n+\mathbb{P})\right)$.

Observe que esta solução consiste em uma atribuição dos vértices em $V \backslash V_{\tau}$ para os vértices em $\tau$. Para isto, denotemos por $V_{1}$ os vértices em $V \backslash V_{\tau}$ com requerimento de recepção maior que 1 e definimos um novo grafo $G^{\prime}$, com novas funções de requerimentos de envio $\sigma^{\prime}$ e recepção $\rho^{\prime}$ e uma nova função de comprimento nas arestas $\omega^{\prime}$, como segue:

- Para cada $u \in V_{1}$ e $i \in\{1, \cdots, \rho(u)\}$, adicionamos o vértice $u_{i}$ ao conjunto $V_{G^{\prime}}$, onde:

$$
\begin{aligned}
& -\sigma^{\prime}\left(u_{i}\right)=\frac{\sigma(u)}{\rho(u)}, \rho^{\prime}\left(u_{i}\right)=1 \mathrm{e} \\
& -\omega^{\prime}\left(u_{i}, v\right)=\frac{(\sigma(u)(\mathbb{P}-\rho(u))+\rho(u)(\mathbb{S}-\sigma(u)))}{\rho(u)\left(\frac{\sigma(u)}{\rho(u)}(\mathbb{P}-1)+\mathbb{S}-\frac{\sigma(u)}{\rho(u)}\right)} \omega(u, v) .
\end{aligned}
$$

- O resto dos vértices (aqueles em $V \backslash V_{1}$ ) são adicionados a $V_{G^{\prime}}$ com os mesmos valores nas funções de requerimento. Também, o resto das arestas são adicionadas a $G^{\prime}$ mantendo o mesmo valor da função de comprimento.

Note que um $k$-centro ótimo $X^{\prime}$ com esqueleto $\tau$ para WSDOCT métrico e instância $\left\langle G^{\prime}, \sigma^{\prime}, \rho^{\prime}, \omega^{\prime}\right\rangle$ garante que para cada $u \in V_{1}$ todos os vértices $u_{i}(i \in\{1, \cdots, \rho(u)\})$ são adjacentes em $X^{\prime}$ do mesmo vértice de $\tau$. Para provar isto, suponha que existem dois vértices $u_{1}$ e $u_{2}$ obtidos a partir de algum $u \in V_{1}$ tais que em $X^{\prime}, u_{1}$ é adjacente de $v_{1} \in V_{\tau}$ e $u_{2}$ é adjacente de $v_{2} \in V_{\tau}$, com $v_{1} \neq v_{2}$. Nesse caso, temos que o custo de comunicação de $X^{\prime}$ é dado por:

$$
\begin{aligned}
C\left(X^{\prime}, \sigma^{\prime}, \rho^{\prime}\right)= & \sum_{u, v \in V_{G^{\prime}} \backslash\left\{u_{1}, u_{2}\right\}} d\left(X^{\prime}, u, v\right)\left(\sigma^{\prime}(u) \rho^{\prime}(v)+\rho^{\prime}(u) \sigma^{\prime}(v)\right) \\
& +\sum_{u \in V_{G^{\prime}} \backslash\left\{u_{1}, u_{2}\right\}} d\left(X^{\prime}, u_{1}, u\right)\left(\sigma^{\prime}\left(u_{1}\right) \rho^{\prime}(u)+\sigma^{\prime}(u)\right) \\
& +\sum_{u \in V_{G^{\prime}} \backslash\left\{u_{1}, u_{2}\right\}} d\left(X^{\prime}, u_{1}, u\right)\left(\sigma^{\prime}\left(u_{2}\right) \rho^{\prime}(u)+\sigma^{\prime}(u)\right) \\
& +\left(\omega\left(u_{1}, v_{1}\right)+\omega\left(u_{2}, v_{2}\right)+d\left(X^{\prime}, u_{1}, u_{2}\right)\right)\left(\sigma^{\prime}\left(u_{1}\right)+\sigma^{\prime}\left(u_{2}\right)\right) .
\end{aligned}
$$

Sem perda de generalidade suponha:

$$
\begin{aligned}
& \sum_{u \in V_{G^{\prime}} \backslash\left\{u_{1}, u_{2}\right\}} d\left(X^{\prime}, u_{1}, u\right)\left(\sigma^{\prime}\left(u_{1}\right) \rho^{\prime}(u)+\sigma^{\prime}(u)\right)+\omega\left(u_{1}, v_{1}\right)\left(\sigma^{\prime}\left(u_{1}\right)+\sigma^{\prime}\left(u_{2}\right)\right) \\
\leq & \sum_{u \in V_{G^{\prime}} \backslash\left\{u_{1}, u_{2}\right\}} d\left(X^{\prime}, u_{2}, u\right)\left(\sigma^{\prime}\left(u_{2}\right) \rho^{\prime}(u)+\sigma^{\prime}(u)\right)+\omega\left(u_{2}, v_{2}\right)\left(\sigma^{\prime}\left(u_{1}\right)+\sigma^{\prime}\left(u_{2}\right)\right) .
\end{aligned}
$$

Se denotamos por $X^{*}$ o $k$-centro com esqueleto $\tau$ obtido após substituir a aresta $\left\langle u_{2}, v_{2}\right\rangle$ pela aresta $\left\langle u_{2}, v_{1}\right\rangle$ em $X^{\prime}$, então: 


$$
\begin{aligned}
C\left(X^{*}, \sigma^{\prime}, \rho^{\prime}\right)= & \sum_{u, v \in V_{G^{\prime}} \backslash\left\{u_{1}, u_{2}\right\}} d\left(X^{\prime}, u, v\right)\left(\sigma^{\prime}(u) \rho^{\prime}(v)+\rho^{\prime}(u) \sigma^{\prime}(v)\right) \\
& +2 \sum_{u \in V_{G^{\prime}} \backslash\left\{u_{1}, u_{2}\right\}} d\left(X^{\prime}, u_{1}, u\right)\left(\sigma^{\prime}\left(u_{1}\right) \rho^{\prime}(u)+\sigma^{\prime}(u)\right) \\
& +2\left(\omega\left(u_{1}, v_{1}\right)\right)\left(\sigma^{\prime}\left(u_{1}\right)+\sigma^{\prime}\left(u_{2}\right)\right) \\
\leq & C\left(X^{\prime}, \sigma^{\prime}, \rho^{\prime}\right)+d\left(X^{\prime}, v_{1}, v_{2}\right)\left(\sigma^{\prime}\left(u_{1}\right)+\sigma^{\prime}\left(u_{2}\right)\right) \\
< & C\left(X^{\prime}, \sigma^{\prime}, \rho^{\prime}\right) .
\end{aligned}
$$

O que é uma contradição pois $X^{\prime}$ é ótima. Portanto, um $k$-centro ótimo $X^{\prime}$ com esqueleto $\tau$ para a instância $\left\langle G^{\prime}, \sigma^{\prime}, \rho^{\prime}, \omega^{\prime}\right\rangle$ satisfaz que para cada $u \in V_{1}$ todos os vértices $u_{i}\left(i \in\left\{1, \cdots, r_{r}(u)\right\}\right)$ são adjacentes em $X^{\prime}$ do mesmo vértice $v \in V_{\tau}$, logo, substituindo em $X^{\prime}$ todos os vértices $u_{i}$ $\left(i \in\left\{1, \cdots, r_{r}(u)\right\}\right)$ por $u$ obtemos um $k$-centro $X$ com esqueleto $\tau$ que é uma solução para o WSDOCT métrico com instância $\langle G, \sigma, \rho, \omega\rangle$, sendo o custo de comunicação de $X$ o mesmo que o de $X^{\prime}$. Na outra direção, é fácil ver que todo $k$-centro $X$ com esqueleto $\tau$ para a instância $\langle G, \sigma, \rho, \omega\rangle$, tem associado um $k$-centro $X^{\prime}$ com esqueleto $\tau$ para a instância $\left\langle G^{\prime}, \sigma^{\prime}, \rho^{\prime}, \omega^{\prime}\right\rangle$, que é obtido substituindo cada vértice $u \in V_{1}$ pelos vértices $u_{i}\left(i \in\left\{1, \cdots, r_{r}(u)\right\}\right)$ e colocando-os como adjacentes do vértice $v \in \tau$ que é adjacente de $u$ em $X$, tendo $X$ e $X^{\prime}$ o mesmo custo de comunicação. Com isto concluímos que o $k$-centro $X$ obtido a partir do $k$-centro ótimo $X^{\prime}$ é um $k$-centro ótimo com esqueleto $\tau$ para o WSDOCT métrico com instância $\langle G, \sigma, \rho\rangle$.

Na próxima seção mostramos como encontrar em tempo $O\left(n^{2 k} \log ^{2}(n)\right)$ um $k$-centro ótimo com esqueleto $\tau$ para o WSDOCT métrico quando os requerimentos de recepção são binários, isto é, $\rho(u) \in\{0,1\}$ para todo $u \in V \backslash V_{\tau}$ que é o caso de $\rho^{\prime}$, onde $n\left(G^{\prime}\right) \leq n+\mathbb{P}$. Com isto provamos o lema seguinte.

Lema 6.4. Dadas uma instância $I=\langle G, \sigma, \rho, \omega\rangle$ do WSDOCT métrico e uma sub-árvore $\tau$ de $G$, se todo vértice $u \in V \backslash V_{\tau}$ satisfaz $\rho(u) \in \mathbb{N}$, então um $k$-centro ótimo com esqueleto $\tau$ para I pode ser encontrado em tempo $O\left((n+\mathbb{P})^{2 k} \log ^{2}(n+\mathbb{P})\right)$, onde $k=n(\tau)$.

\section{3 $k$-centro ótimo com requerimentos de recepção binários nas fo- lhas}

Nesta seção mostramos como encontrar de forma eficiente um $k$-centro ótimo com esqueleto $\tau$ para uma instância do WSDOCT métrico e uma sub-árvore $\tau$ de $G$, onde os requerimentos de recepção são binários para todos os vértices fora de $\tau$, isto é $\rho(u) \in\{0,1\}$ para cada $u \in V_{G \backslash \tau}$.

Para isto, introduzimos o conceito de configuração binária de um $k$-centro.

Definição 6.5. Dado um k-centro $T=(\tau, S)$ de $G$, se para cada $u \in V_{G \backslash \tau}$ o requerimento de recepção é binário, então denotemos para $u \in V_{\tau}$ por $S_{u}^{0}$ o conjunto de vértices com requerimento de recepção zero em $S_{u}$ e $S_{u}^{1}$ o conjunto de vértices com requerimento de recepção um. Logo, definimos por uma configuração binária de T a tupla $\left(\tau, L^{0}, L^{1}\right)$ onde $L^{0}=\left\{l_{u}^{0}\right\}_{u \in V_{\tau}}$ e $L^{1}=\left\{l_{u}^{1}\right\}_{u \in V_{\tau}}$ são duas sequências de $k$ inteiros cada tais que $l_{u}^{0}=\left|S_{u}^{0}\right|$ e $l_{u}^{1}=\left|S_{u}^{1}\right|$. Similar ao conceito de configuração, uma configuração binária $\left(\tau, L_{0}, L_{1}\right)$ está sobre $(k, G)$, se $k$ é um inteiro positivo, $G$ é um grafo, 
$\tau$ é uma sub-árvore de $G$ com $k$ vértices e $L_{0}$ e $L_{1}$ duas sequências de inteiros não negativos que satisfazem $\sum_{u \in V_{\tau}} l_{u}^{0}=n_{0}$ e $\sum_{u \in V_{\tau}} l_{u}^{1}=n_{1}$, onde $n_{0}$ e $n_{1}$ são os números de vértices em $V_{G \backslash \tau}$ com requerimento de recepção zero e um, respectivamente, observe que $n_{0}+n_{1}=n-k$.

A proposta é enumerar por todas as configurações encontrando uma solução ótima para cada configuração o que é feito reduzindo o problema ao UMCF (definido adiante); para finalmente selecionar a solução com menor custo de comunicação entre as encontradas.

O número de configurações binárias sobre $(k, G)$, dada uma sub-árvore $\tau$ a ser esqueleto da configuração, resulta $O\left(n^{2 k-2}\right)$. Este valor é calculado pelo número de possibilidades de atribuir valores não negativos que somem no máximo $n-k$ às $k$ posições de $L^{0}\left(O\left(n^{k-1}\right)\right)$ e $L^{1}\left(O\left(n^{k-1}\right)\right)$.

Proposição 6.6. Dadas uma instância $I=\langle G, \omega, \sigma, \rho, k\rangle$ do problema do $k$-centro ótimo para $o$ WSDOCT métrico, onde $\rho: V_{G \backslash \tau} \leftarrow\{0,1\}$, e uma configuração binária $c=\left(\tau, L^{0}, L^{1}\right)$ sobre $(k, G)$, o problema de encontrar um $k$-centro ótimo com configuração c para I pode ser reduzido em tempo polinomial para o UMCF com instância $I^{\prime}=\left\langle G^{\prime}, \omega^{\prime}, \beta^{\prime}\right\rangle$, onde:

- $V_{G^{\prime}}=V_{G-\tau} \cup\left\{v_{0}\right\}_{v \in V_{\tau}} \cup\left\{v_{1}\right\}_{v \in V_{\tau}}$;

- $E_{G^{\prime}}=\left\{\left(u, v_{\rho(u)}\right) \mid u \in V_{G \backslash \tau} \wedge v \in \tau\right\}$;

- $\operatorname{Se} \rho(u)=1$

$$
\omega^{\prime}\left(u, v_{1}\right)=\mathbb{S} \omega(u, v)+\sum_{w \in V_{\tau}} \sigma(u)(d(\tau, v, w)+\omega(u, v))\left(l_{w}^{1}+\rho(w)\right)-2 \sigma(u) \omega(u, v) ;
$$

- $S e \rho(u)=0$

$\omega^{\prime}\left(u, v_{0}\right)=\sum_{w \in V_{\tau}} \sigma(u)(d(\tau, v, w)+\omega(u, v))\left(l_{w}^{1}+\rho(w)\right) ;$

- Para cada $u \in V_{G \backslash \tau}, \beta^{\prime}(u)=-1$;

- Para cada $v \in V_{\tau}, \beta^{\prime}\left(v_{0}\right)=l_{u}^{0}$ e $\beta^{\prime}\left(v_{1}\right)=l_{u}^{1}$.

Note que o grafo $G^{\prime}$ tem um vértice por cada vértice de $G$ fora de $\tau$, e dois por cada vértice de $G$ em $\tau$ (um para associar com as folhas que tem requerimento de recepção igual a 1 e outro para as que tem requerimento de recepção igual a 0 ). Se o vértice $u$ fora de $\tau$, tem requerimento de recepção igual a 1 , haverá um arco em $G^{\prime}$ entre ele e cada vértice $v_{1}$ (para $v \in V_{\tau}$ ), sendo o custo do arco $\left\langle u, v_{1}\right\rangle$ equivalente ao custo de comunicação que representa colocar o vértice $u \in V_{T \backslash \tau}$ como adjacente de $v \in V_{\tau}$ em um $k$-centro com a configuração dada. Similarmente, se o requerimento de recepção de $u$ é igual a zero, então haverá um arco em $G^{\prime}$ entre ele e cada vértice $v_{0}$ (para $v \in V_{\tau}$ ). O custo dos arcos em $G^{\prime}$ considera o custo do requerimento de envio de $u$ para cada vértice $w \in V_{\tau}$, dado pela multiplicação com os requerimentos de recepção, isto é $\sigma(u)$ vezes $\left(l_{w}^{1}+\rho(w)\right.$ ) (que inclui a transmissão com o vértice $w$ junto as folhas adjacentes a ele); considerando também, no caso do vértice $u$ ter custo de recepção igual a 1 , o custo do requerimento de recepção de $u$ para cada vértice de $G$, isto é $(\mathbb{S}-\sigma(u))$, que chega até $u$ passando pelo vértice $v$. Finalmente as demandas $\beta^{\prime}$ são definidas para garantir uma atribuição entre os vértices fora de $\tau$ com os vértices definidos a partir daqueles em $\tau$.

Demonstração. Primeiro precisamos definir formalmente o UMCF: 
Problema 6.7. UMCF - Uncapacitated Minimum Cost Flow problem

Entrada: Um grafo dirigido $G$, uma função de custo sobre os arcos de $G \omega: E \rightarrow \mathbb{Q}_{+}$e uma funçẫo de demandas sobre os vértices de $G \beta: V \rightarrow \mathbb{Z}$.

Saída: Um vetor de inteiros $X=\left(x_{e}\right)_{e \in E}$ indexados pelos arcos de $G$ que minimize o custo $C(X)=\sum_{e \in E} \omega(e) x_{e}$ e ainda garanta para cada vértice $u \in V$ :

$\sum_{e \in \delta^{+}(u)} x_{e}-\sum_{e \in \delta^{-}(u)} x_{e}=\beta(u)$, onde $e \in \delta^{+}(w)$ e e $\in \delta^{-}(v)$ se e somente se, $e=\langle v, w\rangle(\forall e \in E, v, w \in V)$.

Como as demandas em $I^{\prime}$ são inteiras temos que em qualquer solução viável do UMCF o valor de cada $x_{e}$ será zero ou um. De fato, exatamente $n-k \operatorname{arcos}$ de $G^{\prime}$ terão valor associado 1 . Isto garante que toda solução viável $S^{\prime}$ do problema de fluxo representa uma atribuição entre as folhas fora de $\tau$ e os vértices em $\tau$, definindo a qual vértice de $\tau$ cada folha será adjacente em um $k$-centro de $G$ com configuração $\left(\tau, L^{0}, L^{1}\right)$ associado à solução $S^{\prime}$.

Por outro lado, dado um $k$-centro $T$ de $G$ com configuração $\left(\tau, L^{0}, L^{1}\right)$, se para cada $v \in \tau$ e para cada folha $u$ adjacente a $v$ em $T$ fazemos $x_{\left\langle u, v_{0}\right\rangle}=1$ caso o requerimento de recepção de $u$ é igual a zero, e $x_{\left\langle u, v_{1}\right\rangle}=1$ caso o requerimento de recepção de $u$ é igual a 1 , então obtemos uma solução viável para o problema de fluxo $S^{\prime}$ associada a $T$.

Desta forma concluímos que toda solução viável para o UMCF tem um $k$-centro de $G$ com configuração $\left(\tau, L^{0}, L^{1}\right)$ associado, e vice-versa.

Agora estabelecemos uma relação entre os custos de uma solução viável do UMCF $S^{\prime}$ e o $k$-centro $T$ de $G$ associado a esta. Para isto, denote por $p(u)$ o vértice em $\tau$ adjacente a $u \in V_{G \backslash \tau}$.

$$
\begin{aligned}
C\left(S^{\prime}\right)= & \sum_{u \in V_{G \backslash \tau}} \omega^{\prime}(u, p(u)) \\
= & \sum_{u \in V_{G \backslash \tau}}\left(\sum_{v \in V_{G}} \sigma(v)-\sigma(u)\right) \rho(u) \omega(u, p(u)) \\
& +\sum_{u \in V_{G \backslash \tau}} \sum_{v \in V_{\tau}} \sigma(u) \rho(v)(d(\tau, p(u), v)+\omega(u, p(u))) \\
& +\sum_{u \in V_{G \backslash \tau}} \sum_{v \in V_{G \backslash \tau}-u} \sigma(u) \rho(v)(d(\tau, p(u), p(v))+\omega(u, p(u)+\omega(v, p(v)))) \\
= & \sum_{u \in V_{\tau}} \sum_{v \in V_{\tau}} \sigma(v) \rho(u) d(\tau, u, v)-\sum_{u \in V_{\tau}} \sum_{v \in V_{\tau}} \sigma(v) \rho(u) d(\tau, u, v) \\
& +\sum_{u \in V_{G \backslash \tau}} \sum_{v \in V_{\tau}} \sigma(v) \rho(u) \omega(u, p(u)) \\
& +\sum_{u \in V_{G \backslash \tau}} \sum_{v \in V_{\tau}} \sigma(v) \rho(u) d(\tau, p(u), v)-\sum_{u \in V_{G}-\tau} \sum_{v \in V_{\tau}} \sigma(v) \rho(u) d(\tau, p(u), v) \\
& +\sum_{u \in V_{G \backslash \tau}} \sum_{v \in V_{\tau}} \sigma(u) \rho(v)(d(\tau, p(u), v)+\omega(u, p(u))) \\
& +\sum_{u \in V_{G \backslash \tau}} \sum_{v \in V_{G \backslash \tau}-u} \sigma(u) \rho(v)(d(\tau, p(u), p(v))+\omega(u, p(u))+\omega(v, p(v))) \\
& \sum_{u \in V_{G}} \sum_{v \in V_{G}} \sigma(u) \rho(v) d(S, u, v)-\sum_{u \in V_{\tau}} \sum_{v \in V_{\tau}} \sigma(v) \rho(u) d(\tau, u, v)
\end{aligned}
$$




$$
\begin{aligned}
& -\sum_{u \in V_{G \backslash \tau}} \sum_{v \in V_{\tau}} \sigma(v) \rho(u) d(\tau, p(u), v) \\
= & C(T)-\sum_{u \in V_{\tau}} \sum_{v \in V_{\tau}} \sigma(v) \rho(u) d(\tau, u, v)-\sum_{v \in V_{\tau}} \sum_{u \in V_{\tau}} \sigma(v) d(\tau, u, v) l_{u}^{1} \\
= & C(T)-\sum_{u \in V_{\tau}} \sum_{v \in V_{\tau}} \sigma(v) d(\tau, u, v)\left(l_{u}^{1}+\rho(u)\right) .
\end{aligned}
$$

Observe que para todo $k$-centro com configuração $\left(\tau, L^{0}, L^{1}\right)$ o valor $\sum_{u \in V_{\tau}} \sum_{v \in V_{\tau}} \sigma(v) d(\tau, u, v)\left(l_{u}^{1}+\right.$ $\rho(u))$ é exatamente o mesmo. Logo, uma solução ótima para o UMCF com instância $I^{\prime}$ está associada a um $k$-centro ótimo com configuração $\left(\tau, L^{0}, L^{1}\right)$ do WSDOCT métrico com instância $I$.

Para obter $I^{\prime}$ a partir de $I$ devem ser calculados os custos dos arcos de $G^{\prime}$, o que pode ser feito em tempo $O\left((n-k) k^{3}\right)$. A definição das demandas de $G^{\prime}$ pode ser feito em $O(n)$. O $k$-centro $T$ associado à solução $S^{\prime}$ do UMCF pode ser obtido em tempo $O(n-k)$, e seu custo de comunicação, $C(T)$, em tempo $O\left(k^{3}\right)$. Logo, a redução completa pode ser feita em tempo $O\left(n k^{3}\right)$.

Um resultado bem conhecido é que o UMCF pode ser resolvido em tempo $O(n \log (n)(n k+$ $n \log (n)))=O\left(n^{2} \log ^{2}(n)\right)$ ([Or193]). Logo, é possível encontrar um $k$-centro ótimo com esqueleto $\tau$ de forma eficiente para o WSDOCT métrico com requerimentos de recepção binários nos vértices fora de $\tau$.

Lema 6.8. Dados um inteiro positivo $k$ fixo, e uma sub-árvore $\tau$ com $k$ vértices, o problema do $k$-centro ótimo com esqueleto $\tau$ para o WSDOCT métrico com requerimentos de recepção binários nos vértices fora de $\tau$ pode ser resolvido em tempo $O\left(n^{2 k} \log ^{2}(n)\right)$.

\subsection{Resumo}

Observe que o PTAS que apresentamos neste capítulo para o WSDOCT métrico, melhora a 2-aproximação para o SROCT métrico que até o momento era a melhor razão conhecida para este problema. Ainda, o WSDOCT métrico generaliza quase todas as versões métricas do OCT estudadas na literatura, portanto este resultado generaliza os PTAS encontrados anteriormente na literatura. A seguinte figura, similar aos capítulos anteriores, mostra a relação dos problemas aqui estudados e os melhores resultados de aproximação encontrados para eles. 


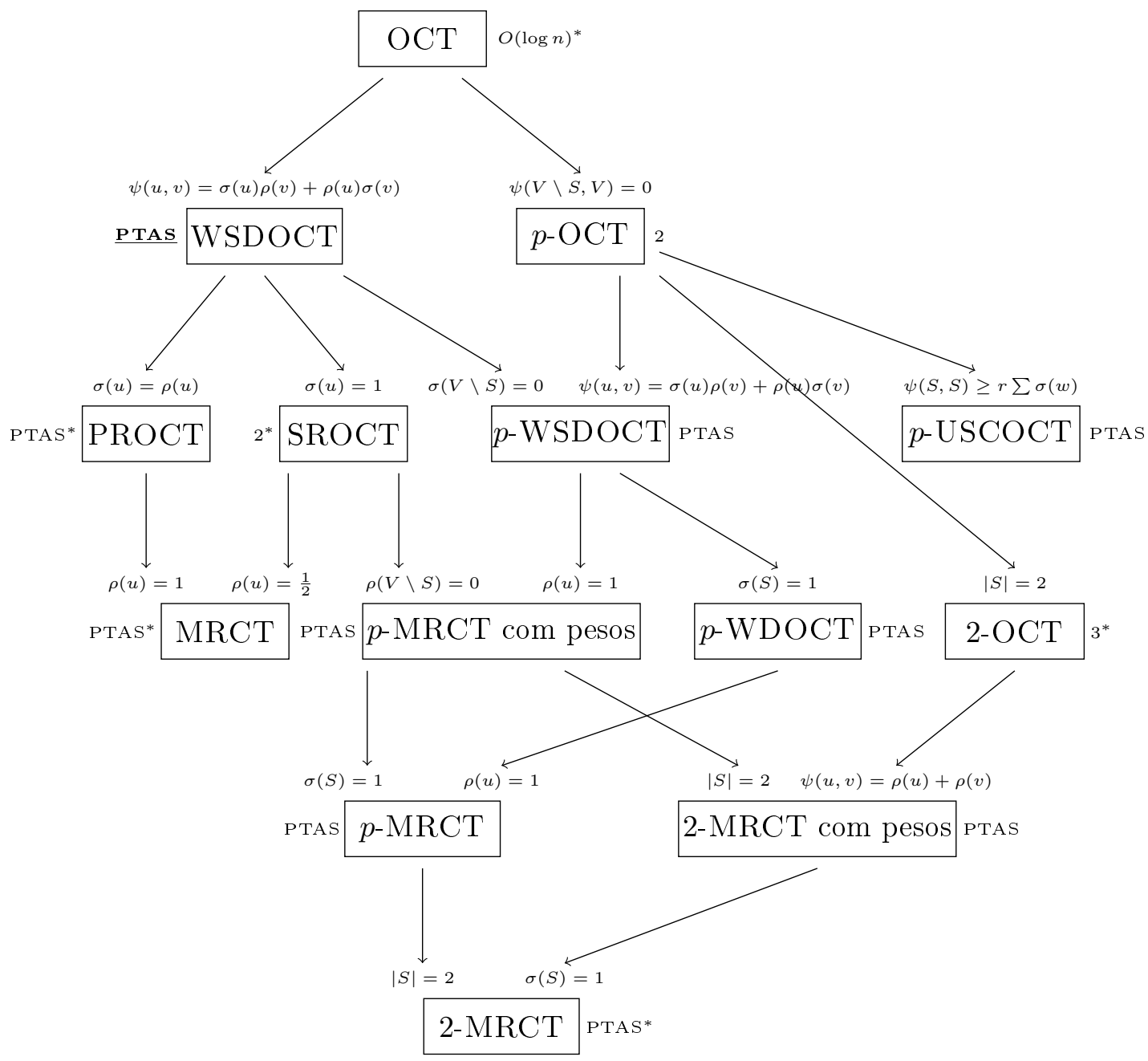

Figura 6.1: Relação entre o $\mathrm{OCT}$ e suas variantes NP-difíceis. Observe que o PTAS que damos para o WSDOCT métrico, generaliza todos os PTAS existentes na literatura para os casos métricos das variantes do OCT. 


\section{Capítulo 7}

\section{Conclusões}

Nesta tese analisamos os resultados existentes para o OCT e suas principais variantes na literatura, apresentamos novos casos particulares para este problema, alguns deles generalizando vários problemas anteriormente estudados e também obtivemos novas aproximações para vários destes problemas, melhorando em alguns casos, a melhor razão de aproximação que existia.

Introduzimos três casos particulares do $p$-OCT ( $p$-USCOCT, $p$-WDOCT e $p$-WSDOCT) mostrando que eles são NP-difíceis, inclusive quando consideradas somente instâncias métricas. Ainda, damos condições suficientes para que casos particulares do p-OCT métrico admitam PTAS e mostramos como aplicar as mesmas na obtenção de PTASs em vários destes problemas, incluindo aqueles que introduzimos. Com isto, generalizamos vários dos resultados obtidos anteriormente na literatura e melhoramos alguns deles. Estes resultados geraram a publicação [RF15b] e o resumo deles é dado no capítulo 4 pela tabela 4.1 .

Também introduzimos o WSDOCT, uma outra variante NP-difícil do OCT que generaliza alguns dos problemas anteriores, além de praticamente todos os que foram estudados na literatura. Nos capítulos 5 e 6 mostramos como obter um PTAS para este problema, o que implica um PTAS para o SROCT, um caso particular dele para o qual a melhor aproximação conhecida era uma 2-aproximação. Este resultado ainda não foi publicado, porém para o caso particular do SROCT, o PTAS que encontramos gerou a publicação [RF15a].

Note que, a menos que $\mathrm{P}=\mathrm{NP}$, nenhum dos problemas tratados neste trabalho admite FPTAS, portanto os PTASs que apresentamos são a melhor razão de aproximação que se pode encontrar para eles. Porém, não são conhecidos outros resultados sobre inaproximabilidade para esta família de problemas, o que deixa muitas questões em aberto sobre o OCT e suas variantes. Por exemplo, será possível generalizar os resultados obtidos para casos métricos se considerarmos grafos gerais? O OCT, seu caso métrico ou o $p$-OCT, admitem alguma razão de aproximação constante? O p-OCT métrico admite um PTAS?

Como há poucos resultados teóricos para estes problemas, são muitas as portas que se abrem para pesquisas futuras. Por exemplo, outra linha de pesquisa sobre o problema, na qual iniciamos um estudo preliminar, é a modelagem como problema de programação linear inteiro, e buscar entender melhor a estrutura de um poliedro associado ao problema. No apêndice A incluímos uma revisão bibliográfica dos modelos de programação linear inteira existentes junto com modelos inéditos para o OCT. 
CONCLUSÕES 


\section{Apêndice A}

\section{Pesquisa a partir de modelos matemáticos}

Uma das possibilidades para futuras linhas de pesquisa é analisar os modelos matemáticos existentes e alguns novos.

A partir deles é possível pensar em algoritmos para o OCT baseados em relaxação lagrangeana, geração de colunas e planos de cortes. O que pode resultar em algoritmos como Branch $\mathcal{E}$ Bound, Branch $\&$ Cost e Branch $\&$ Price que ainda não foram implementados. Outra possibilidade é tentar resultados de aproximação baseados nos modelos matemáticos propostos.

A seguir vemos os modelos apresentados na literatura e depois apresentamos novos modelos matemáticos para o OCT.

\section{A.1 Modelos matemáticos na literatura}

O primeiro modelo de programação linear inteira para o OCT foi proposto em [Rot07]. O modelo usa variáveis binárias para indicar a inclusão de uma aresta na solução assim como para indicar se uma aresta e um vértice estão no caminho entre dois vértices da solução. Segue a descrição deste:

Modelo A.1. :

\section{Dados:}

- $G=\left(V_{G}, E_{G}\right)$, grafo não dirigido com conjunto de vértices $V_{G}$ e conjunto de arestas $E_{G}$;

- $\omega: E_{G} \rightarrow \mathbb{Q}_{+}$, comprimentos associados às arestas de $G$;

- $\psi: V_{G} \times V_{G} \rightarrow \mathbb{Q}_{+}$, requerimento entre cada par orientado de vértices;

- $M \in \mathbb{Q}_{+}$, constante suficientemente grande.

\section{Variáveis:}

$$
\begin{aligned}
x_{e} \in\{0,1\}: & \text { indica se a aresta e é usada } \\
y_{u v}^{e} \in\{0,1\}: & \text { indica se a aresta e pertence ao caminho com origem em u e destino v } \\
z_{u v}^{w} \in\{0,1\}: & \text { indica se o vértice } w \text { está no caminho com origem em u e destino } v
\end{aligned}
$$




\section{Formulação:}

$$
\begin{array}{ll}
\min & \sum_{u \in V_{G}} \sum_{v \in V_{G}} \sum_{e \in E_{G}} \psi(u, v) \omega(e) y_{u v}^{e} \\
\text { s.a: } \quad & \sum_{(u, w) \in E_{G}} y_{u v}^{u w}=1 \quad \forall u, v \in V_{G}, u \neq v \\
& \sum_{(v, w) \in E_{G}} y_{u v}^{v w}=1 \quad \forall u, v \in V_{G}, u \neq v \\
& \sum_{(w, z) \in E_{G}} y_{u v}^{w z}=2 z_{u v}^{w} \quad \forall u, v, w \in V_{G}, u \neq w \neq v \\
& \sum_{u \in V_{G}} \sum_{v \in V_{G}} y_{u v}^{e} \geq x_{e} \quad \forall e \in E_{G} \\
& \sum_{u \in V_{G}} \sum_{v \in V_{G}} y_{u v}^{e} \leq M x_{e} \quad \forall e \in E_{G} \\
& \sum_{(u, v) \in V_{G}} x_{u v} \geq 1 \quad \forall u \in V_{G} \\
& \sum_{e \in E_{G}} x_{u v}=n-1 \\
& x_{u v}, y_{u v}^{w z}, z_{u v}^{w} \in\{0,1\} \quad \forall u, v, w, z \in V_{G}
\end{array}
$$

As restrições (A.2) e (A.3) garantem que na solução para todo par de vértices $u$, $v$, há exatamente uma aresta adjacente a u e uma aresta adjacente a $v$. Evidentemente isto garante a existência de um único começo e um único fim para todos os caminhos de u a $v$ na solução, que juntando-se às restrições (A.4), garante a existência de um único caminho de u para v. As restriçôes (A.5) e (A.6) estabelecem que cada aresta da soluçâo deve estar contida em pelo menos um caminho da solução. As restrições (A.7) garantem que todo vértice deve ter uma aresta adjacente na solução e a restrição (A.8) que a solução tenha exatamente $n-1$ arestas, assim se garante que a solução seja uma árvore. Finalmente, as restrições (A.9) obrigam que todas as variáveis na solução tomem valores binários.

Em [Con09] foram dados três modelos para o OCT, um deles com uma formulação baseada em fluxo e os outros dois em caminhos. Neles é feita uma distinção entre arestas, arcos e links, onde as definições de arestas e arcos são as usuais e link é um par de vértices $(u, v)$ com requerimento de comunicação de $u$ para $v$ maior do que zero (isto é $\psi(u, v)>0$ ). Considere então o conjunto de arcos de um grafo $G$ como $A_{G}$ e o conjunto de links como $L_{G}$. O primeiro destes modelos é:

\section{Modelo A.2. :}

Dados:

- $G=\left(V_{G}, E_{G}\right)$, grafo não dirigido com conjunto de vértices $V_{G}$ e conjunto de arestas $E_{G}$;

- $\omega: E_{G} \rightarrow \mathbb{Q}_{+}$, comprimentos associados às arestas de $G$;

- $\psi: V_{G} \times V_{G} \rightarrow \mathbb{Q}_{+}$, requerimento entre cada par orientado de vértices;

- $L_{G}$, conjunto de links de $G$. 
Variáveis:

$x_{u}^{v w} \in \mathbb{Q}_{+}: \quad$ quantidade de fluxo que tem origem em u e passa pelo arco $(v, w)$ $y_{u v} \in\{0,1\}: \quad$ indica se a aresta $(u, v)$ está na solução

\section{Formulação:}

$$
\begin{array}{ll}
\min & \sum_{u \in V_{G}} \sum_{v \in V_{G}} \sum_{w \in V_{G}-v} w(v, w) x_{u}^{v w} \\
\text { s.a: } & \sum_{w \in V_{G}-v} x_{u}^{w v}-\sum_{w \in V_{G}-v} x_{u}^{v w}=\psi(u, v) \quad \forall(u, v) \in L_{G} \\
& x_{u}^{v w}+x_{u}^{w v} \leq y_{v w} \sum_{z \in V_{G}} \psi(u, z) \quad \forall u \in V_{G},(v w) \in E_{G} \\
& \sum_{u v \in E_{G}} y_{u v}=n-1 \\
& x_{u}^{v w} \geq 0 \quad \forall u, v, w \in V_{G}, v \neq w \\
& y_{u v} \in\{0,1\} \quad \forall(u, v) \in E_{G}
\end{array}
$$

Onde a restrição (A.11) garante que chegue em $v$ o requerimento de comunicação do vértice $u$. A restrição (A.12) garante que somente pode haver transferência pelos arcos que se encontram na solução e a (A.13) garante que sejam usados exatamente $n-1$ arestas.

O segundo modelo em [Con09] também usado em [CFM10] resulta:

\section{Modelo A.3. :}

\section{Dados:}

- $G=\left(V_{G}, E_{G}\right)$, grafo não dirigido com conjunto de vértices $V_{G}$ e conjunto de arestas $E_{G}$;

- $\omega: E_{G} \rightarrow \mathbb{Q}_{+}$, comprimentos associados às arestas de $G$;

- $\psi: V_{G} \times V_{G} \rightarrow \mathbb{Q}_{+}$, requerimento entre cada par orientado de vértices;

- $L_{G}$, conjunto de links de $G$.

Variáveis:

$$
\begin{array}{ll}
x_{u v}^{r} \in\{0,1\}: & \text { indica se o arco }(u, v) \text { está no caminho do link } r \\
y_{u v} \in\{0,1\}: & \text { indica se a aresta }(u, v) \text { está na solução }
\end{array}
$$

\section{Formulação:}

$$
\min \sum_{r \in L_{G}} \sum_{v \in V_{G}} \sum_{w \in V_{G}-v} \psi(r) \omega(v, w) x_{v w}^{r}
$$




$$
\begin{array}{ll}
\text { s.a: } & \sum_{w \in V_{G}-u} x_{u w}^{r}=1 \quad \forall r=(u, v) \in L_{G} \\
& \sum_{w \in V_{G}-v} x_{w v}^{r}-\sum_{w \in V_{G}-v} x_{v w}^{r}=0 \quad \forall r \in L_{G}, v \in V_{G} \\
& x_{v w}^{r}+x_{w v}^{r} \leq y_{v w} \quad \forall r \in L_{G},(v w) \in E_{G} \\
& \sum_{u v \in E_{G}} y_{u v}=n-1 \\
& x_{u v}^{r} \geq 0 \quad \forall u, v \in V_{G}, u \neq v, r \in L_{G} \\
& y_{u v} \in\{0,1\} \quad \forall(u, v) \in E_{G}
\end{array}
$$

Observe que as restrições (A.17) e (A.18) garantem a conservação de fluxo sobre cada link, fazendo também com que a solução defina uma estrutura conexa sobre os vértices. O resto das restriçôes são semelhantes às do modelo A.2.

O terceiro modelo apresentado em [Con09] é:

\section{Modelo A.4. :}

\section{Dados:}

- $G=\left(V_{G}, E_{G}\right)$, grafo não dirigido com conjunto de vértices $V_{G}$ e conjunto de arestas $E_{G}$;

- $\omega: E_{G} \rightarrow \mathbb{Q}_{+}$, comprimentos associados às arestas de $G$;

- $\psi: V_{G} \times V_{G} \rightarrow \mathbb{Q}_{+}$, requerimento entre cada par orientado de vértices;

- $L_{G}$, conjunto de links de $G$;

- $P_{r}$, conjunto de caminhos dirigidos em $G$ de u a $v$ (para cada link $r=(u, v) \in L_{G}$ ).

\section{Variáveis:}

$$
\begin{aligned}
z_{p}^{r} \in\{0,1\}: & \text { indica se o caminho } p \text { é usado para atender o link } r \\
y_{u v} \in\{0,1\}: & \text { indica se a aresta }(u, v) \text { está na solução }
\end{aligned}
$$

\section{Formulação:}

$$
\begin{array}{ll}
\min & \sum_{r \in L_{G}} \sum_{p \in P_{r}} \psi(r) \omega(p) z_{p}^{r} \\
\text { s.a: } & \sum_{p \in P_{r}} z_{p}^{r}=1 \quad \forall r \in L_{G} \\
& \sum_{p:(u, v) \in E_{p}} z_{p}^{r} \leq Y_{u v} \quad \forall r \in L_{G},(u, v) \in E_{G} \\
& \sum_{u v \in E_{G}} y_{u v}=n-1 \\
& z_{p}^{r} \in\{0,1\} \quad \forall r \in L_{G}, p \in P_{r} \\
& y_{u v} \in\{0,1\} \quad \forall(u, v) \in E_{G}
\end{array}
$$


Note que as restriçôes (A.24) garantem que exatamente um caminho seja selecionado para cada link e (A.25) que unicamente caminhos com as arestas na solução serão considerados para cada link. Sendo o resto das restrições similares às usadas nos modelos A.2 e A.3.

\section{A.2 Novos modelos matemáticos}

Agora, formulamos novos modelos matemáticos para o OCT.

O modelo A.5 considera o conjunto $P_{G}$ de todos os caminhos simples em $G$, sendo $P_{u v}$ o conjunto de todos os caminhos em $G$ que conetam os vértices $u$ e $v$.

\section{Modelo A.5. :}

\section{Dados:}

- $G=\left(V_{G}, E_{G}\right)$, grafo não dirigido com conjunto de vértices $V_{G}$ e conjunto de arestas $E_{G}$;

- $\omega: E_{G} \rightarrow \mathbb{Q}_{+}$, comprimentos associados às arestas de $G$;

- $\psi: V_{G} \times V_{G} \rightarrow \mathbb{Q}_{+}$, requerimento entre cada par orientado de vértices;

- $P_{u v}$, conjunto de todos os caminhos em $G$ que conetam os vértices u e v (para todo $u, v \in V_{G}$ );

- $P_{G}$, conjunto de todos os caminhos de $G$;

- $S_{p}$, conjunto de todos os caminhos de $G$ que contêm p (para cada caminho $p$ de G).

\section{Variáveis:}

$$
x_{p} \in\{0,1\}: \quad \text { indica se o caminho } p \text { está na solução }
$$

\section{Formulação:}

$$
\begin{array}{ll}
\min & \sum_{u \in V_{G}} \sum_{v \in V_{G}} \sum_{p \in P_{u v}} \psi(u, v) \omega(p) x_{p} \\
\text { s.a: } & \sum_{p \in P_{u v}} x_{p}=1 \quad \forall u, v \in V_{G}, u \neq v \\
& \sum_{q \in S_{p}} x_{q} \leq\left|S_{P}\right| x_{p} \quad \forall p \in P_{G} \\
& x_{p} \in\{0,1\} \quad \forall p \in P_{G}
\end{array}
$$

Note que as restriçôes (A.30) definem que entre todo par de vértices deve existir um único caminho, com isto se garante a conexidade que junto às restriçôes (A.31) elimina a existência de circuitos na solução. As restriçôes (A.32) garantem que a solução tenha valores binários. Acreditamos que seja possivel aplicar técnicas de geração de colunas usando este modelo.

Perceba que a ideia do modelo A.5 é similar à do modelo A.4, a principal diferença está nas restrições usadas por cada modelo para garantir que a solução além de conexa seja acíclica. Isto é feito no modelo A.4 usando as restrições (A.25) e (A.26), onde se estabelece uma relação entre 
os valores das variáveis associadas aos caminhos e as associadas às arestas, exigindo que a solução tenha $n-1$ arestas; enquanto no modelo A.5 são usadas as restrições da forma (A.31), o que faz desnecessário nele o uso de variáveis binárias associadas às arestas do grafo.

A seguir mostramos outra formulação que usa ideias parecidas às empregadas no modelo A.1, porém apresenta um conjunto diferente de restrições para garantir a conexidade e relacionar as variáveis associadas às arestas com as associadas aos caminhos, obtendo um modelo que acreditamos ser mais simples porém com um número exponencial de restrições.

Modelo A.6. :

\section{Dados:}

- $G=\left(V_{G}, E_{G}\right)$, grafo não dirigido com conjunto de vértices $V_{G}$ e conjunto de arestas $E_{G}$;

- $\omega: E_{G} \rightarrow \mathbb{Q}_{+}$, comprimentos associados às arestas de $G$;

- $\psi: V_{G} \times V_{G} \rightarrow \mathbb{Q}_{+}$, requerimento entre cada par orientado de vértices;

- $P_{u v}$, conjunto de todos os caminhos em $G$ que conetam os vértices u e $v$ (para todo $u, v \in V_{G}$ ).

\section{Variáveis:}

$x_{e} \in\{0,1\}: \quad$ indica se a aresta e está na solução

$y_{u v}^{e} \in\{0,1\}: \quad$ indica se na solução o caminho entre o vértice u e o $v$ passa pela aresta $e$

\section{Formulação:}

$$
\begin{array}{ll}
\min & \sum_{u \in V_{G}} \sum_{v \in V_{G}} \sum_{e \in E_{G}} \psi(u, v) \omega(e) y_{u v}^{e} \\
\text { s.a: } \quad & \left|E_{p}\right|-\sum_{f \in E_{p}} x_{f} \geq 1-y_{u v}^{e} \quad \forall u, v \in V_{G}, e \in E_{G}, p \in\left\{q \mid e \in E_{q} \wedge q \in P_{u v}\right\} \\
& \sum_{e \in E_{G}} y_{u v}^{e} \geq 1 \quad \forall u, v \in V_{G}, u \neq v \\
& y_{u w}^{e}+y_{u v}^{e}+y_{v w}^{e} \leq 2 x_{e} \quad \forall u, v, w \in V_{G}, e \in E_{G} \\
& \sum_{e \in E_{G}} x_{e}=n-1 \\
& x_{e}, y_{u v}^{e} \in\{0,1\} \quad \forall u, v \in V_{G}, e \in E_{G}
\end{array}
$$

Observe que as restrições (A.34) obrigam a que se na solução existe um caminho entre u e $v$ que passa pela aresta e, então $y_{u v}^{e}=1$. As restrições (A.35) e (A.36) garantem que para todo par de vértices exista uma aresta que os separe, com isto se garante a conexidade, enquanto as restrições (A.37) garantem que no máximo há $n-1$ arestas na solução, desta forma, se obtem uma estrutura de árvore. As restrições (A.38) garantem que a solução tenha valores binários. Acreditamos que seja possivel aplicar técnicas de planos de corte usando este modelo.

No modelo abaixo, mostramos uma formulação baseada em cortes. Dado um conjunto $X \subseteq V_{G}$, denotamos por $\partial(X)$ as arestas com uma ponta em $X$ e outra em $V_{G}-X$. 
Modelo A.7. :

\section{Dados:}

- $G=\left(V_{G}, E_{G}\right)$, grafo não dirigido com conjunto de vértices $V_{G}$ e conjunto de arestas $E_{G}$;

- $\omega: E_{G} \rightarrow \mathbb{Q}_{+}$, comprimentos associados às arestas de $G$;

- $\psi: V_{G} \times V_{G} \rightarrow \mathbb{Q}_{+}$, requerimento entre cada par orientado de vértices;

- $\partial: 2^{V_{G}} \rightarrow 2^{E_{G}}$, corte associado a cada subconjunto de $V_{G}$.

Variáveis:

$$
\begin{aligned}
x_{e} \in\{0,1\}: & \text { indica se a aresta e está na solução } \\
y_{u v}^{e} \in\{0,1\}: & \text { indica se a aresta e pertence a um caminho entre os vértices u ev }
\end{aligned}
$$

\section{Formulação:}

$$
\begin{array}{ll}
\min & \sum_{u \in V_{G}} \sum_{v \in V_{G}} \sum_{e \in E_{G}} \psi(u, v) \omega(e) y_{u v}^{e} \\
\text { s.a: } \quad & \sum_{e \in \partial(X)} x_{e} \geq 1 \quad \forall \phi \subset X \subset V_{G} \\
& \sum_{e \in E_{G}} x_{e}=n-1 \\
& y_{u v}^{u v}=x_{u v} \quad \forall(u, v) \in E_{G} \\
& y_{u w}^{e}+x_{w v}-y_{u w}^{w v} \leq 1+y_{u v}^{w v} \quad \forall e,(w, v) \in E_{G} \\
& y_{u w}^{e}+x_{w v}-y_{u w}^{w v} \geq 2 y_{u v}^{w v} \quad \forall e,(w, v) \in E_{G} \\
& y_{u w}^{e}+y_{u v}^{e}+y_{v w}^{e} \leq 2 x_{e} \quad \forall u, v, w \in V_{G}, e \in E_{G} \\
& x_{e}, y_{u v}^{e} \in\{0,1\} \quad \forall u, v \in V_{G}, e \in E_{G}
\end{array}
$$

As restrições (A.40) garantem a conexidade da solução, enquanto a restrição (A.41) garante que é uma árvore geradora. Sendo as restrições (A.42), (A.43), (A.44) e (A.45) as que estabelecem a relação entre as variáveis $x_{e}$ e as $y_{u v}^{e}$, garantindo que se uma aresta está na solução então ela está no caminho entre os vértices adjacentes a ela (restrições (A.42)); que se na solução há um caminho $P$ entre dois vértices $u$ e $v$, e uma aresta que não está em $P$, adjacente a um dos extremos de $P$ (digamos $u$, sendo a aresta $(u, w)$ ), então $(u, w)$ se encontra no caminho entre os vértices $v$ e $w$ (restriçôes (A.43) e (A.44)). O resto das restrições são similares às do modelo A.6. Observe que o número de restriçôes deste modelo é exponencial, contudo acreditamos que também seja possivel aplicar técnicas de planos de corte usando este modelo. 
APÊNDICE A 


\section{Referências Bibliográficas}

[AH73] D. Adolphson e T. C. Hu. Optimal linear ordering. SIAM J. Appl. Math., 25:403-423, 1973. 5

[AM87a] R. K. Ahuja e V. V. S. Murty. Exact and heuristic algorithms for the optimum communication spanning tree problem. Transp Sci, 21:163-170, 1987. 2, 3

[AM87b] R. K. Ahuja e V. V. S. Murty. New lower planes for the network design problem. Networks, 17:113-127, 1987. 2, 3

[Bar96] Y. Bartal. Probabilistic approximation of metric spaces and its algorithmic applications. In Proceedings of the 37th Annual IEEE Symposium on Foundations of Computer Science, páginas 184-196, 1996. 32

[Bar98] Y. Bartal. On approximating arbitrary metrics by tree metrics. Proceedings of the 30th Annual ACM Symposium on Theory of Computer, páginas 161-168, 1998. 32

[CCGG98] M. Charikar, C. Chekuri, A. Goel e S. Guha. Rounding via trees: Deterministic approximation algorithms for group steiner trees and k-median. Proceedings of the 30th Annual ACM Symposium on Theory of Computer, páginas 114-123, 1998. 32

[CFM10] I. Contreras, E. Fernández e A. Marín. Lagrangean bounds for the optimum communication spanning tree problem. TOP, 18:140-157, 2010. 2, 3, 91

[Con09] I. Contreras. Network hub location: models, algorithms and related problems. PhD thesis, Department of Statistics and Operations Research, Technical University of Catalonia, Spain, 2009. 2, 3, 90, 91, 92

[FF57] L. R. E. Ford e D. R. Fulkerson. A simple algorithm for finding maximal network flows and an application to the Hitchcock problem. Ibid, 9:210-218, 1957. 4

$\left[\mathrm{FFK}^{+} 00\right]$ A. M. Farley, P. Fragopoulou, D. Krumme, A. Proskurowski e D. Richards. Multi-source spanning tree problems. Journal of Interconnection Networks, 1(1):61-71, 2000. 2, 3, 15,24

[GH61] R. E. Gomory e T. C. Hu. Multi-terminal network flows. SIAM J. Appl. Math., 9:551570, 1961. 4

[GJ79] M. R. Garey e D. S. Jhonson. Computers and Intractability: a guide to the theory of NP-completeness. Freeman, 1979. 7

[Hu74] T. C. Hu. Optimum communication spanning trees. SIAM J. Comput., 3(3):188-195, 1974. 1,3

[JLRK78] D. S. Johnson, J. K. Lenstra e A. H. G. Rinnooy Kan. The complexity of the network design problem. Networks, 8:279-285, 1978. 1, 2, 3, 7, 8

[KRS97] G. Konjevod, R. Ravi e F. S. Salman. On approximating planar metrics by tree metrics. manuscript, 1997. 32 
[Or193] B. J. Orlin. A faster strongly polynomial minimum cost flow algorithm. Operations Research, 41(2):338-350, 1993. 84

[RF15a] S. V. Ravelo e C. E. Ferreira. A PTAS for the metric case of the minimum sumrequirement communication spanning tree problem. First International Conference, CALDAM: Algorithms and Discrete Applied Mathematics, páginas 9-20, 2015. 87

[RF15b] S. V. Ravelo e C. E. Ferreira. PTAS's for some metric p-source communication spanning tree problems. 9th International Workshop, WALCOM: Algorithms and Computation, páginas $137-148,2015.87$

[Rot07] F. Rothlauf. Design and applications of metaheuristics. Habilitationsschrift, Universität Mannheim, 2007. 3, 89

[Rot09] F. Rothlauf. On optimal solutions for the optimal communication spanning tree problem. Operations Research, 57:413-425, 2009. 3

[Sha06] P. Sharma. Algorithms for the optimum communication spanning tree. Annals of Operations Research, 143:203-209, 2006. 3

[TFR03] K. Talwar, J. Fakcharoenphol e S. Rao. A tight bound on approximating arbitrary metrics by tree metrics. Proceedings of the 35th Annual ACM Symposium on Theory of Computing, páginas 448-455, 2003. 32

[Wat95] M. S. Waterman. Introduction to Computational Biology. Chapman \& Hall / CRC, 1995. 1

[WCT00a] B. Y. Wu, K. M. Chao e C. Y. Tang. Approximation algorithms for some optimum communication spanning tree problems. Discrete Applied Mathematics, 102:245-266, 2000. $3,20,31,33,37,40,50,51,75,77$

[WCT00b] B. Y. Wu, K. M. Chao e C. Y. Tang. A polynomial time approximation scheme for optimal product-requirement communication spanning trees. J. Algorithms, 36:182204, 2000. 3, 31, 33, 37, 40, 51, 75, 77

$\left[\mathrm{WLB}^{+} 98\right]$ B. Y. Wu, G. Lancia, V. Bafna, K. M. Chao, R. Ravi e C. Y. Tang. A polynomial time approximation scheme for minimum routing cost spanning trees. SIAM on Computing, 29(3):761-778, 1998. 1, 2, 3, 11, 20, 31, 37, 40, 51, 75

[Wu02] B. Y. Wu. A polynomial time approximation scheme for the two-source minimum routing cost spanning trees. J. Algorithms, 44:359-378, 2002. 2, 3, 15, 31, 37, 50

[Wu04] B. Y. Wu. Approximation algorithms for the optimal p-source communication spanning tree. Discrete Applied Mathematics, 143:31-42, 2004. 3, 15, 31, 37, 50 